University of Louisville

ThinkIR: The University of Louisville's Institutional Repository

$12-2013$

\title{
Generation and detection of defects in metallic parts fabricated by selective laser melting and electron beam melting and their effects on mechanical properties.
}

Haijun Gong

University of Louisville

Follow this and additional works at: https://ir.library.louisville.edu/etd

\section{Recommended Citation}

Gong, Haijun, "Generation and detection of defects in metallic parts fabricated by selective laser melting and electron beam melting and their effects on mechanical properties." (2013). Electronic Theses and Dissertations. Paper 515.

https://doi.org/10.18297/etd/515

This Doctoral Dissertation is brought to you for free and open access by ThinkIR: The University of Louisville's Institutional Repository. It has been accepted for inclusion in Electronic Theses and Dissertations by an authorized administrator of ThinkIR: The University of Louisville's Institutional Repository. This title appears here courtesy of the author, who has retained all other copyrights. For more information, please contact thinkir@louisville.edu. 


\title{
GENERATION AND DETECTION OF DEFECTS IN METALLIC PARTS \\ FABRICATED BY SELECTIVE LASER MELTING AND ELECTRON BEAM \\ MELTING AND THEIR EFFECTS ON MECHANICAL PROPERTIES
}

\author{
By \\ Haijun Gong \\ A Dissertation \\ Submitted to the Faculty of the \\ J. B. Speed School of Engineering of the University of Louisville \\ in Partial Fulfillment of the Requirements \\ for the Degree of \\ Doctor of Philosophy \\ Department of Industrial Engineering \\ University of Louisville \\ Louisville, Kentucky
}

December 2013 
Copyright 2013 by Haijun Gong

All rights reserved 



\section{GENERATION AND DETECTION OF DEFECTS IN METALLIC PARTS \\ FABRICATED BY SELECTIVE LASER MELTING AND ELECTRON BEAM MELTING AND THEIR EFFECTS ON MECHANICAL PROPERTIES}

By

Haijun Gong

A Dissertation Approved on

December 3, 2013

by the following Dissertation Committee:

Dissertation Director: Dr. Brent E. Stucker

Dr. Thomas L. Starr

Dr. John S. Usher

Dr. William E. Biles

Dr. Lihui Bai 
To My Families 


\section{ACKNOWLEDGMENTS}

I am grateful to my advisor, Dr. Brent Stucker, for his generous guidance and support during my study and research over the past four years. I would like to thank other committee members, Dr. Thomas Starr, Dr. John Usher, Dr. William Biles, and Dr. Lihui Bai, for their constructive advice during my research at University of Louisville.

I would like to express my gratitude to the staff of the Industrial Engineering Department, whose assistance and encouragement are a valuable resource for the accomplishment of my studies. I would like to thank all the fellows in my research group for their support and friendship. Special thanks go to Dr. Khalid Rafi, Dr. Yanzhe Yang, Dr. John Obielodan, and Dr. Deepankar Pal.

I am thankful to the staff of the Rapid Prototyping Center at the University of Louisville for giving professional technical support to my experiments. Dr. Michael Voor and Mr. Seid Waddell in the Department of Orthopaedic Surgery of the University of Louisville are sincerely thanked for assisting with defect detection and model reconstruction.

This research project was financially supported by the Office of Naval Research through awards N00014-09-1-0147 and N00014-10-1-0800.

Last but not the least, I would like to acknowledge my families for their endless support, encouragement, patience and understanding.

Haijun Gong 


\begin{abstract}
GENERATION AND DETECTION OF DEFECTS IN METALLIC PARTS

FABRICATED BY SELECTIVE LASER MELTING AND ELECTRON BEAM

MELTING AND THEIR EFFECTS ON MECHANICAL PROPERTIES

Haijun Gong
\end{abstract}

December 3, 2013

\begin{abstract}
Application of Additive Manufacturing (AM) technology to fabricate complex three-dimensional components is one promising direction within the manufacturing industry. This approach is rapidly changing the way designers and engineers create objects with desired shape and structures. Selective Laser Melting (SLM) and Electron Beam Melting (EBM) are two common powder bed fusion processes within AM for fabricating metallic parts. In order to give designers and engineers more insights into employing AM, the quality and long-term behavior of SLM- and EBM-produced parts need to be carefully investigated. Thus, this research project aims to understand how processing parameters affect defect generation and distribution during SLM and EBM processes, to study the morphological features of defects, to identify effective non-destructive method(s) to detect these defects, and to characterize the effect of defects on mechanical properties of SLMand EBM-produced parts.
\end{abstract}

The study began by generating stochastic defects via adjustment to process parameters from optimal parameters to marginal parameters, in order to correlate the 
porosity to the marginal parameters. Archimedes method was employed to estimate porosity of SLM- and EBM-produced specimens. After this, by using destructive characterization techniques, the defective specimens were sectioned. The morphology of stochastic defects was investigated based on their contour features on the cross sections. Micro CT was primarily used to evaluate the stochastic defects in the SLM and EBM parts and demonstrate their morphological characteristics and porosity in the single slices and reconstructed models. Finally, tensile and fatigue tests were carried out on Ti-6Al-4V parts with identified porosity. The fracture mechanism was analyzed.

This study established a fundamental understanding of defects in parts made by SLM and EBM processes. Porosity was quantitatively correlated to the marginal parameters of SLM and EBM processes. Defects were differentiated based on their morphological properties and features. Micro CT was confirmed to be an effective nondestructive method for evaluating stochastic defects in SLM- and EBM-produced parts. The effects of stochastic defects on Ti-6Al-4V parts were determined based on tensile and fatigue tests. It was found that both microstructure and porosity have an impact on the mechanical properties of SLM- and EBM-produced parts. 


\section{TABLE OF CONTENTS}

\section{PAGE}

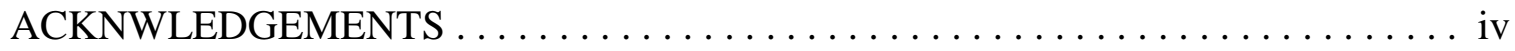

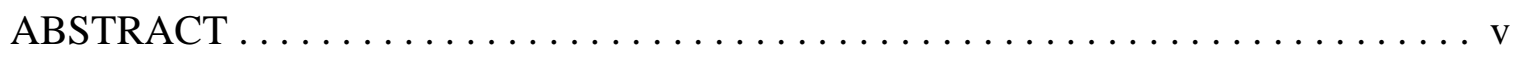

LIST OF TABLES $\ldots \ldots \ldots \ldots \ldots \ldots \ldots \ldots \ldots \ldots \ldots \ldots \ldots \ldots \ldots \ldots \ldots \ldots \ldots \ldots \ldots$

LIST OF FIGURES $\ldots \ldots \ldots \ldots \ldots \ldots \ldots \ldots \ldots \ldots \ldots \ldots \ldots \ldots \ldots \ldots \ldots \ldots \ldots \ldots \ldots$

CHAPTER 1. INTRODUCTION AND LITERATURE REVIEW $\ldots \ldots \ldots \ldots \ldots \ldots 1$

1.1. Research Motivation and Problem Statement .................... 1

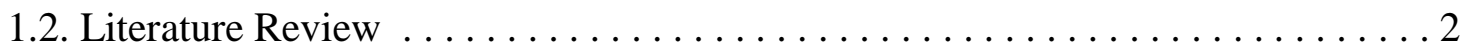

1.2.1. Additive Manufacturing . ............................. 3

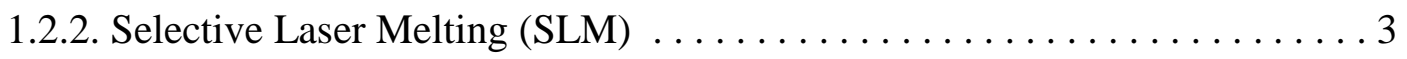

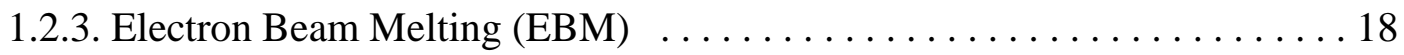

1.2.4. Melting Mechanism of Metallic Powder .................... 26

1.2.5. Defect Detection via Non-destructive Testing (NDT) $\ldots \ldots \ldots \ldots \ldots 27$

1.2.6. Effect of Defects on Mechanical Properties ................... 31

1.2.7. Conclusions from Literature Review $\ldots \ldots \ldots \ldots \ldots \ldots \ldots \ldots \ldots$

1.3. Research Goal and Objectives ........................... 35

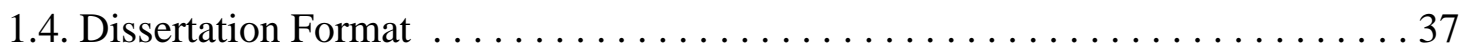

CHAPTER 2. THE EFFECTS OF PROCESSING PARAMETERS ON DEFECT REGULARITY IN TI-6AL-4V PARTS FABRICATED BY SLM AND EBM . . . . . . 39

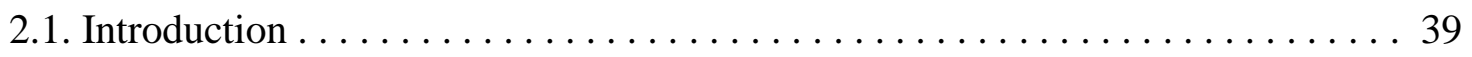




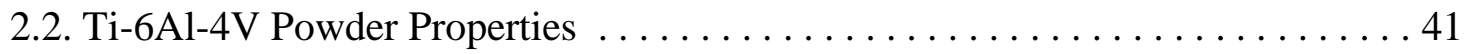

2.2.1. Raymor Ti-6Al-4V Powder for SLM $\ldots \ldots \ldots \ldots \ldots \ldots \ldots \ldots \ldots 42$

2.2.2. Arcam Ti-6Al-4V powder for EBM . ................... 42

2.3. Design of Experiments . . . . . . . . . . . . . . . . . . . . . 43

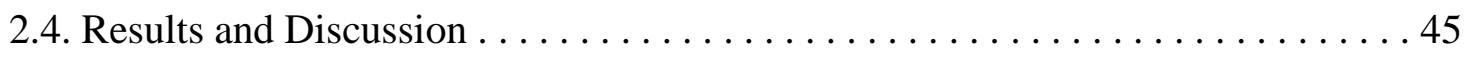

2.4.1. Effect of Processing Parameters on SLM Ti-6Al-4V .............. 45

2.4.2. Effect of Processing Parameters on EBM Ti-6Al-4V . ............. 54

2.4.3. Comparison of Defect Formation between SLM and EBM .......... 60

2.5. Conclusion ....................................61

CHAPTER 3. DEFECT MORPHOLOGY IN TI-6AL-4V PARTS FABRICATED BY

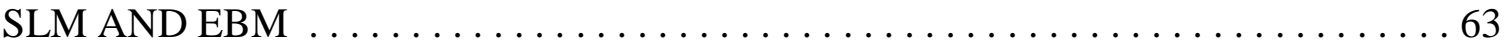

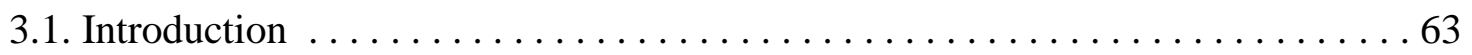

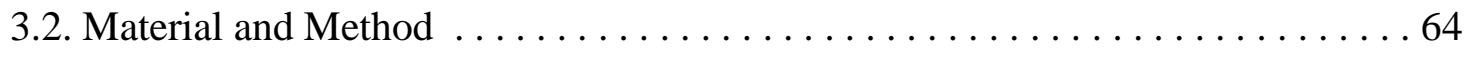

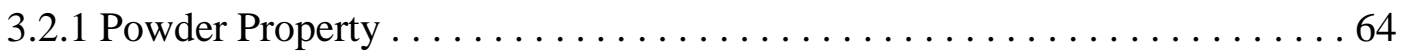

3.2.2 Fabrication of Ti-6Al-4V Specimens $\ldots \ldots \ldots \ldots \ldots \ldots \ldots \ldots \ldots 65$

3.2.3 Image Processing Method .......................... 68

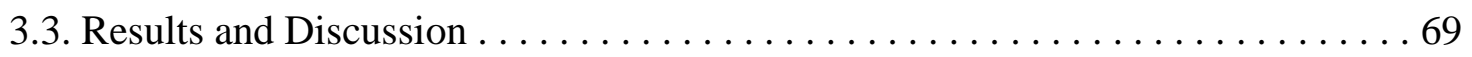

3.3.1 Characterization of SLM Defects $\ldots \ldots \ldots \ldots \ldots \ldots \ldots \ldots \ldots \ldots \ldots$

3.3.2 Characterization of EBM Defects $\ldots \ldots \ldots \ldots \ldots \ldots \ldots \ldots \ldots$

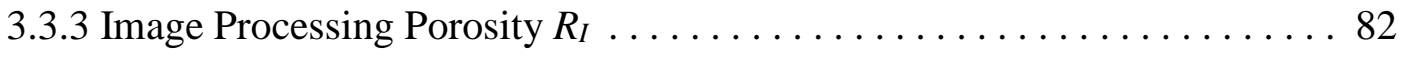

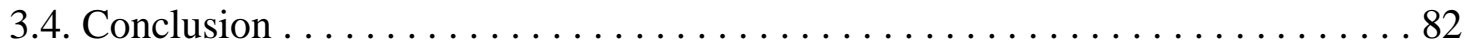

CHAPTER 4. MICRO-CT EVALUATION OF STOCHASTIC DEFECTS

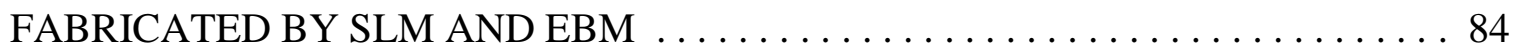




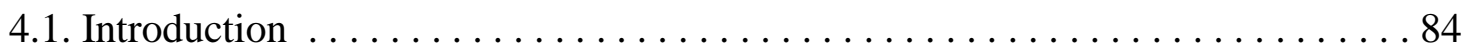

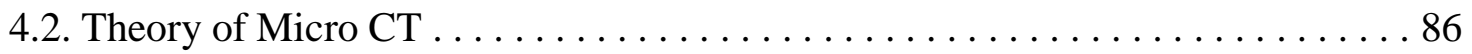

4.3. Experimental Procedures . ............................... 87

4.3.1 Specimen Fabrication ............................... 87

4.3.2 Defects Inspection by Micro CT $\ldots \ldots \ldots \ldots \ldots \ldots \ldots \ldots \ldots$

4.4. Results and Discussion ............................... 89

4.4.1 Characteristics of SLM specimens . . . . . . . . . . . . . . . . 89

4.4.2 Characteristics of EBM specimens ..................... 92

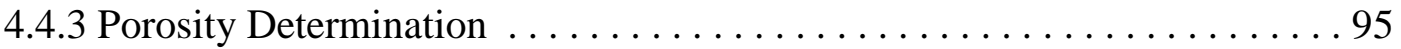

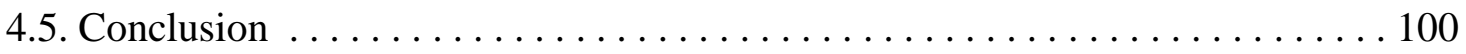

CHAPTER 5. MECHANICAL PROPERTIES OF SLM- AND EBM-PRODUCED TI-

6AL-4V WITH DEFECTS . . . . . . . . . . . . . . . . . . . . . . . . . . . . . . 102

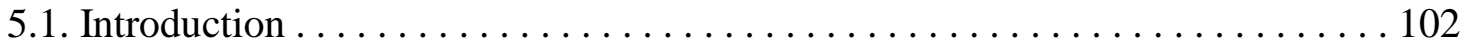

5.2. Materials and Methods ................................. 105

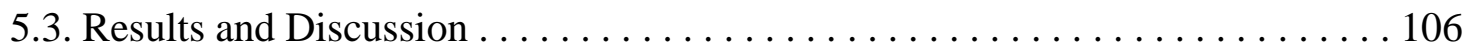

5.3.1 Microstructure ................................. 106

5.3.2 Tensile Properties . . . . . . . . . . . . . . . . . . . . . 107

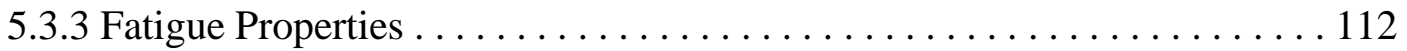

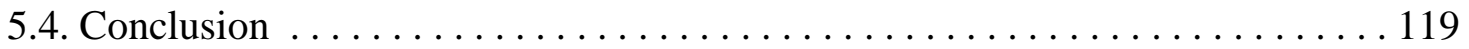

CHAPTER 6. CONCLUSIONS AND FUTURE WORK ................. 121

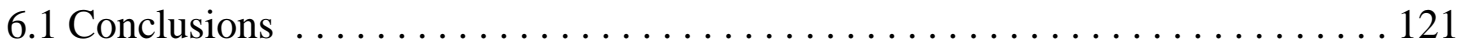

6.1.1 The Effects of Processing Parameters on Defect Generation ........... 121

6.1.2 Defect Morphology in SLM and EBM specimens ............... 123 
6.1.3 Micro-CT Evaluation of Stochastic Defects . . . . . . . . . . . . . 124

6.1.4 Mechanical Properties of Ti-6Al-4V Parts with Defects . . . . . . . . . . 125

6.2 Future Work . . . . . . . . . . . . . . . . . 126

REFERENCE ..................................... 129

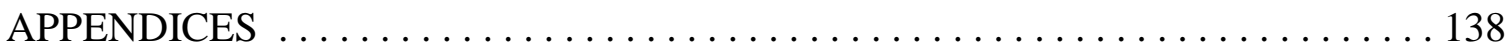

APPENDIX A. DETECTION TO DETERMINISTIC DEFECTS IN SLMPRODUCED PARTS VIA DESTRUCTIVE CHARACTERIZATION TECHNIQUE AND NON-DESTRUCTIVE TESTING METHODS . . . . . . . . . . . . . . 139

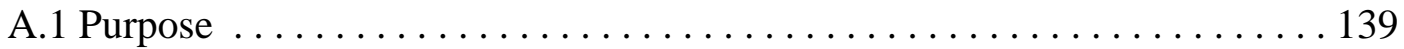

A.2 Sample Fabrication . . . . . . . . . . . . . . . . . . . . . 139

A.3 Defects Detection via Destructive Characterization Technique . . . . . . . . 141

A.4 Defects Detection via NDT Methods . . . . . . . . . . . . . . . . . . . . . 142

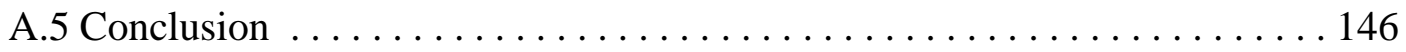

APPENDIX B. EFFECT OF DEFECTS ON FATIGUE TESTS OF AS-BUILT TI6AL-4V PARTS FABRICATED BY SELECTIVE LASER MELTING . . . . . . . . 148

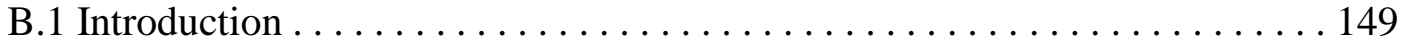

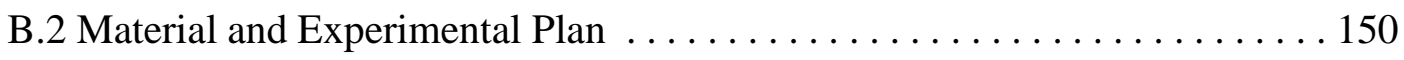

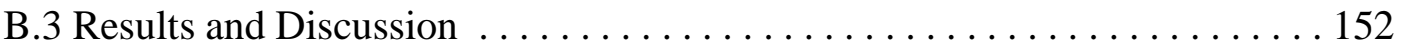

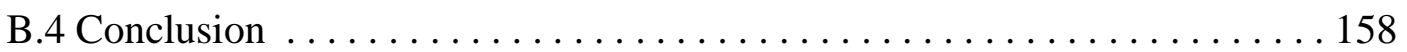

APPENDIX C. MICROSTRUCTURES AND MECHANICAL PROPERTIES OF TI6AL4V PARTS FABRICATED BY SELECTIVE LASER MELTING AND ELECTRON BEAM MELTING . . . . . . . . . . . . . . . 161

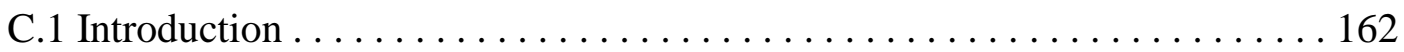




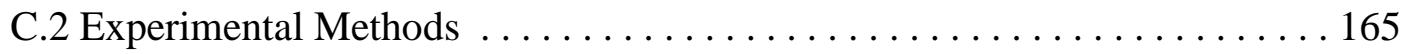

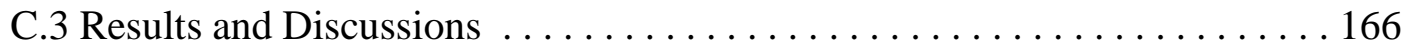

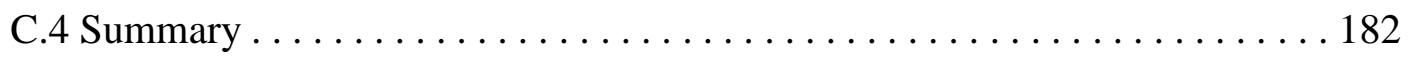

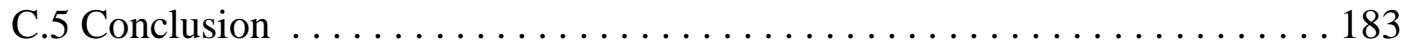

APPENDIX D. PERMISSIONS $\ldots \ldots \ldots \ldots \ldots \ldots \ldots \ldots \ldots \ldots \ldots \ldots \ldots \ldots$

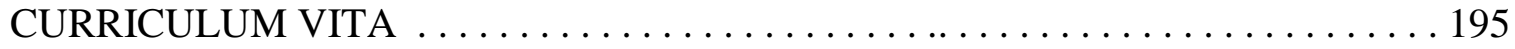




\section{LIST OF TABLES}

PAGE

1.1. Optimized Process Parameters Regarding Part Density and Process Speed for

Titanium and Cobalt-Chromium (Vandenbroucke et al, 2007) $\ldots \ldots \ldots \ldots \ldots 8$

1.2. Results of Mechanical Tests on SLM Samples Compared to Mechanical

Properties of Bulk Material from Literature (Vandenbroucke et al, 2007) ....... 17

1.3. Difference between EBM and SLM (Gibson et al, 2009) .............. 20

2.1. Factors and Levels of Factorial Experiment (SLM: Ti-6Al-4V, Hatch Spacing $100 \mu \mathrm{m}$, Layer Thickness $30 \mu \mathrm{m}) \ldots \ldots \ldots \ldots \ldots \ldots \ldots \ldots \ldots \ldots \ldots \ldots \ldots$

2.2. Factors and Levels of Taguchi Experiment (EBM: Ti-6Al-4V) ............ 44

2.3. ANOVA of Factorial Experiment (trimmed) $\ldots \ldots \ldots \ldots \ldots \ldots \ldots \ldots \ldots \ldots$

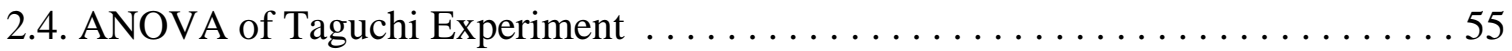

4.1. Marginal Parameters of SLM and EBM $\ldots \ldots \ldots \ldots \ldots \ldots \ldots \ldots \ldots$

5.1. Experimental Parameters for SLM Test Specimens (Laser Power 120W, Hatch

Spacing $0.1 \mathrm{~mm}$, Layer Thickness $0.03 \mathrm{~mm}) \ldots \ldots \ldots \ldots \ldots \ldots \ldots \ldots \ldots \ldots \ldots$

5.2. Experimental Parameters for EBM Test Specimens (Layer Thickness 0.05mm) . . 106

5.3. Tensile Properties of SLM- and EBM-Produced Ti-6Al-4V Specimens ....... 109

A.1. Formability of Cylindrical Defects in the SLM-Produced Specimen ......... 141

A.2. Formability of Conical Defects in the SLM-Produced Specimen ............ 141

B.1. Process Parameters for Fabricating Ti-6Al-4V Fatigue Specimens $\ldots . \ldots \ldots \ldots 151$

C.1. Tensile Results for SLM Produced and EBM Produced Ti64 Alloy Samples . . . . 175 


\section{LIST OF FIGURES}

PAGE

1.1 Schematic of SLM process (Thijs et al, 2010) $\ldots \ldots \ldots \ldots \ldots \ldots \ldots \ldots \ldots$

1.2 Process map for continuous laser melting operation (Kruth et al, 2004) ....... 7

1.3 Results of parameter study for Ti-6Al-4V (Vandenbroucke et al, 2007) ....... 8

1.4 Schematic of the scanning strategy investigated by Morgan et al (2002) . . . . . 12

1.5 The six different scanning strategies compared by Kruth et al (2004) ......... 12

1.6. Schematic of three scanning strategies (Thijs et al, 2010) $\ldots \ldots \ldots \ldots \ldots \ldots$

1.7 Schematic of EBM process (Murr et al, 2009a) ..................... 19

1.8 Scan strategies of EBM (Lu et al, 2009) ....................... 22

1.9 Stress-strain curves for Ti-6Al-4V (Facchini et al, 2010) . . . . . . . . . . . 25

1.10. Experimental setup for thermography inspection (www.visiooimage.com) $\ldots . .29$

1.11 Schematic of ultrasonic testing (www.virtualengg.com) $\ldots \ldots \ldots \ldots \ldots \ldots 30$

1.12 Schematic of radiography testing (www.energyworkforce.net) $\ldots \ldots \ldots \ldots . \ldots 30$

1.13 Bending strength and relative density for different laser regimes $\ldots \ldots \ldots \ldots 32$

1.14 Results of hardness analysis for Ti-6Al-4V4V (Vandenbroucke et al, 2007) ..... 33

2.1 Schematic of SLM process (source: EOS basic training manual) $\ldots \ldots \ldots \ldots \ldots 40$

2.2 Schematic of EBM process (Murr et al, 2009) ................... 40

2.3 Raymor Ti-6Al-4V powder morphology and particle size distribution $\ldots \ldots \ldots \ldots 42$

2.4 Arcam Ti-6Al-4V powder morphology and particle size distribution $\ldots \ldots \ldots \ldots 43$

2.5 Porosity of SLM-produced Ti-6Al-4V specimen $\ldots \ldots \ldots \ldots \ldots \ldots \ldots \ldots . \ldots 46$ 
2.6 Porosity distribution of SLM factorial experiment $\ldots \ldots \ldots \ldots \ldots \ldots \ldots \ldots$

2.7 Process window for SLM Raymor Ti-6Al-4V powder $\ldots \ldots \ldots \ldots \ldots \ldots \ldots . . \ldots 8$

2.8 Scanning electron microscopy of top surface of laser melted Ti-6Al-4V . . . . . 50

2.9 SEM of pits and particles on top surface of SLM specimen $\ldots \ldots \ldots \ldots \ldots \ldots \ldots 1$

2.10 Schematic of defect caused by recoating during SLM process $\ldots \ldots \ldots \ldots \ldots 2$

2.11 Schematic of defect daused by Zone III marginal parameters of SLM process.

(a) Laser melting metallic powder; (b) Top view of two adjacent hatch lines . . . . 53

2.12 Top surface fabricated by Zone III marginal parameters of SLM process . . . . . 54

2.13 Mean porosity at each level of taguchi experimental factors $\ldots \ldots \ldots \ldots \ldots 54$

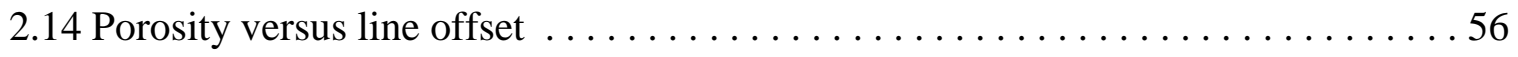

2.15 Solidified top surfaces of EBM specimens by varying line offset $\ldots \ldots \ldots \ldots 57$

2.16 Schematic of melt pool with increased line offset during EBM process . . . . . 57

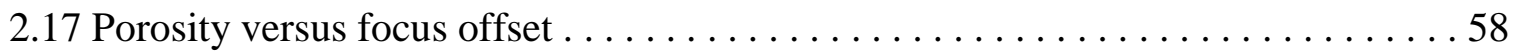

2.18 Solidified top surfaces of EBM specimens by varying line offset $\ldots \ldots \ldots \ldots 59$

2.19 Schematic of melting pool with increased focus offset during EBM process . . . . 59

3.1 Process window of SLM and porosity distribution at $120 \mathrm{~W}$ and $80 \mathrm{~W} \ldots \ldots \ldots 6$

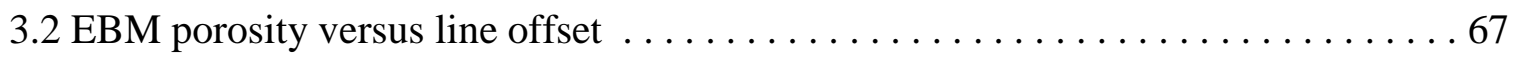

$3.3 \mathrm{EBM}$ porosity versus focus offset $\ldots \ldots \ldots \ldots \ldots \ldots \ldots \ldots \ldots \ldots \ldots \ldots \ldots \ldots \ldots \ldots \ldots$

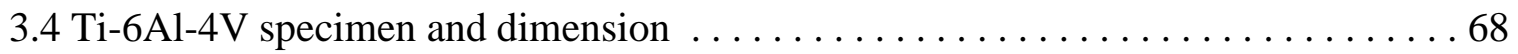

3.5 Cross section of SLM-produced Zone II specimens $\ldots \ldots \ldots \ldots \ldots \ldots \ldots \ldots .70$

3.6 Distribution of defect morphology of SLM-produced Zone II specimen . . . . . . . 71

3.7 Cross section of SLM-produced Zone III specimens $\ldots \ldots \ldots \ldots \ldots \ldots \ldots \ldots$

3.8 Distribution of defect morphology of SLM-produced Zone III specimen . . . . . . 73 
3.9 Comparison of cross section of SLM-produced specimen $\ldots \ldots \ldots \ldots \ldots \ldots 74$

3.10 Distribution of SLM Zone II and III defect morphology (porosity $\approx 5.8 \%$ ) . . . . . 75

3.11 Top surfaces of SLM specimens by marginal parameters (a) Zone II; and

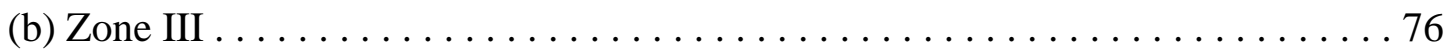

3.12 Cross sections of EBM-produced specimen at various line offset values . . . . . . 77

3.13 Distributions of $L O$ defect morphology of EBM-produced specimens . . . . . . . 78

3.14 Cross sections of EBM-produced specimen at various focus offset values $\ldots \ldots 80$

3.15 Distribution of $F O$ defect morphology of EBM-produced specimens $\ldots \ldots \ldots 80$

3.16 Top surface of EBM specimens (a) $L O=0.26 \mathrm{~mm}$ and (b) $F O=20 \mathrm{~mA} \ldots \ldots \ldots 81$

4.1 Schematic of SLM process (source: EOS basic training manual) $\ldots \ldots \ldots \ldots \ldots 8$

4.2 Schematic of EBM process (Murr et al, 2009a) .................... 85

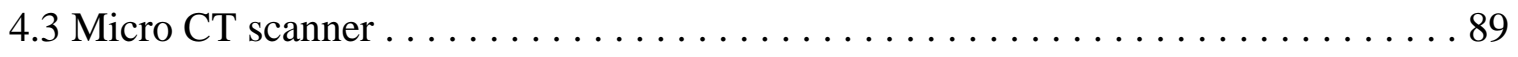

4.4 Single slices and locally reconstructed models of SLM specimens (Zone II). (a)

$V=360 \mathrm{~mm} / \mathrm{s}, R_{D}=6.0 \%$. (b) $V=480 \mathrm{~mm} / \mathrm{s}, R_{D}=2.0 \%$. (c) $V=600 \mathrm{~mm} / \mathrm{s}, R_{D}=0.5 \% \ldots 90$

4.5 Single slices and locally reconstructed models of SLM specimens (Zone III). (a)

$V=1200 \mathrm{~mm} / \mathrm{s}, R_{D}=0.1 \%$. (b) $V=1320 \mathrm{~mm} / \mathrm{s}, R_{D}=2.0 \%$. (c) $V=1560 \mathrm{~mm} / \mathrm{s}$,

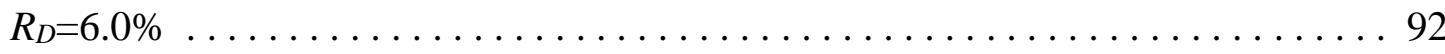

4.6 Single slices and locally reconstructed models of EBM specimens. (a) $L O=0.18$ mm, $R_{D}=0.7 \%$. (b) $L O=0.24 \mathrm{~mm}, R_{D}=2.0 \%$. (c) $L O=0.30 \mathrm{~mm}, R_{D}=4.0 \% \ldots \ldots .93$

4.7 Single slices and locally reconstructed models of EBM specimens. (a) $F O=16 \mathrm{~mA}$, $R_{D}=0.3 \%$. (b) $F O=20 \mathrm{~mA}, R_{D}=3.0 \%$. (c) $F O=24 \mathrm{~mA}, R_{D}=4.5 \% \ldots \ldots \ldots \ldots$

4.8 Image processing of a single slice of a CT scanned EBM specimen . ......... 96

4.9 Schematic of grayscale adjustment to an individual cell (16 pixels) ......... 97 
4.10 Example of image correction and binary image $\ldots \ldots \ldots \ldots \ldots \ldots \ldots . \ldots 9$

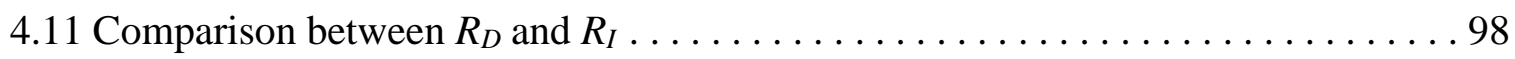

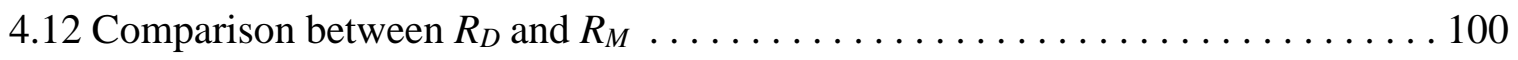

5.1 Optical microstructure. (a) Horizontal cross-section of SLM specimen; (b) vertical cross-section of SLM specimen; (c) horizontal cross-section of EBM specimen;

(d) vertical cross-section of EBM specimen $\ldots \ldots \ldots \ldots \ldots \ldots \ldots \ldots \ldots \ldots \ldots$

5.2 Stress-strain plots of SLM- and EBM-produced Ti-6Al-4V specimens $\ldots \ldots \ldots 108$

5.3 SEM fractographs of tensile specimens. (a) SLM (porosity 1\%, Zone II); (b)

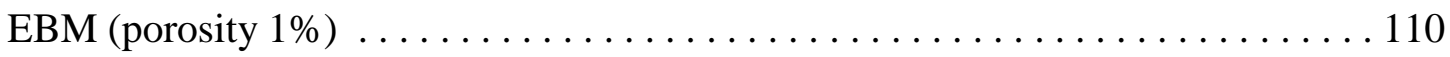

5.4 SEM fractographs of tensile specimens (a) SLM (porosity 5\%, Zone II); (b) enlarged view from the boxed region in '(a) ' $\ldots \ldots \ldots \ldots \ldots \ldots \ldots \ldots \ldots \ldots$

5.5 SEM fractographs of the tensile specimens. (a) SLM 1 (porosity 1\%, Zone III);

(b) enlarged view from the boxed region in '(a)'; (c) EBM (porosity 5\%); (d)

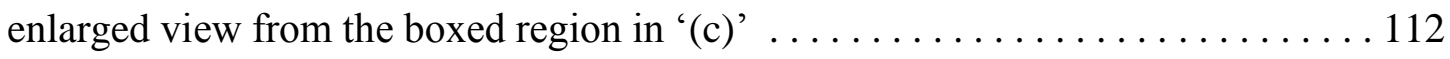

5.6 Fatigue life of SLM-produced Ti-6Al-4V specimen $(R=0.1$, load controlled smooth

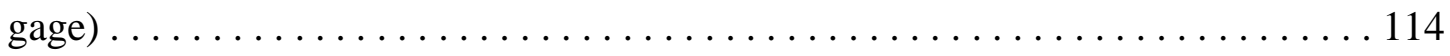

5.7. Fatigue fractography of SLM-produced fatigue specimens by SLM-OP1.

(a)Fracture surface; (b) enlarged view in '(a)' ........................ 114

5.8 Fatigue fractography of SLM-produced fatigue specimens. (a) Fracture surface (porosity $1 \%$, Zone II); (b) enlarged view in '(a)'; (c) fracture surface (porosity

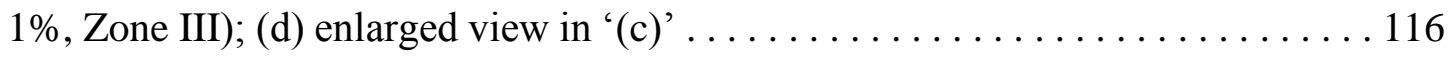

5.9 Fatigue life of EBM-produced Ti-6Al-4V specimens $(R=0.1$, load controlled Smooth gage) 
5.10 Fatigue fractography of EBM-produced fatigue specimens. (a) Fracture surface (porosity 0\%); (b) enlarged view in '(a); (c) fracture surface (porosity 5\%); (d)

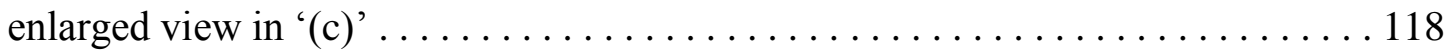

A.1 SLM-produced Specimen for NDT. (a) CAD file; (b) As-built specimen . . . . . . 140

A.2 UT transducer ......................................... 143

A.3 UT A-scan result ..................................... 144

A.4 SLM-produced fatigue specimen for CT scan $\ldots \ldots \ldots \ldots \ldots \ldots \ldots \ldots \ldots$

A.5 CAD models and reconstructed inspection results from CT scanning images .... 145

B.1 EOS Ti-6Al-4V powder morphology and particle size distribution $\ldots \ldots \ldots \ldots 150$

B.2 CAD model of fatigue specimen. (a) Cylindrical defect; (b) D-conical defect . . . 151

B.3 As-built Ti-6Al-4V fatigue specimen and its surface feature $\ldots \ldots \ldots \ldots \ldots . \ldots 152$

B.4 Stress-life plot for fatigue testing of Ti64 specimens with and without geometric

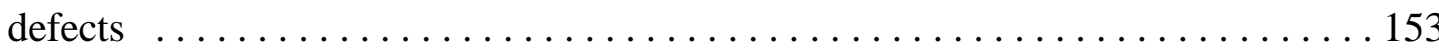

B.5 Fractography of fatigue specimen (crack initiated from gauge surface). (a) Top View; (b) Crack initiation site; (c) Crack propagation (transgranular fracture); (d) Final fast fracture region; (e) Fatigue striation in crack propagation region (SEM);

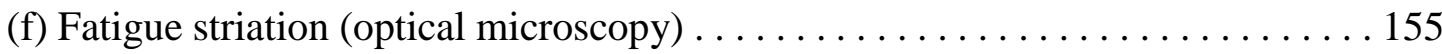

B.6 Fracture surface of fatigue specimen (crack initiated from central cylindrical

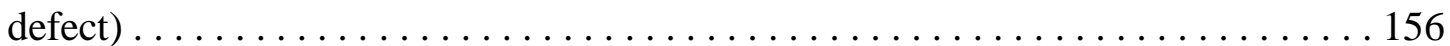

B.7 Fracture surface of fatigue specimen (crack initiated from central double-conical

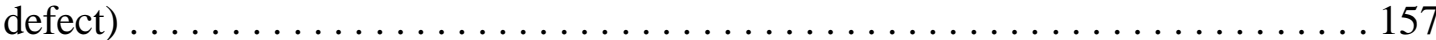

C.1 (a) Powder size distribution of EOS supplied Ti64 powder (Avg particle size: 36 $\mu \mathrm{m}$ ); (b) Powder size distribution of Arcam supplied Ti64 powder (Avg particle 
size: $60 \mu \mathrm{m}$ ); (c) SEM-SE image of EOS supplied Ti64 powder; (d) SEM-SE image of Arcam supplied Ti64 powder $\ldots \ldots \ldots \ldots \ldots \ldots \ldots \ldots \ldots \ldots \ldots . \ldots \ldots$

C.2 a) As-built tensile samples produced in SLM; b) As-built tensile samples produced

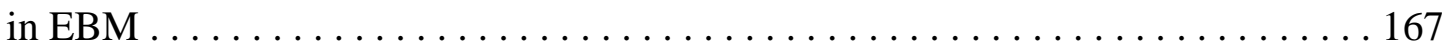

C.3 a) External surface of a vertically built SLM sample (arrow shows the build direction); b) External surface of a horizontally built SLM sample (build direction is perpendicular to the image plane); c) External surface of a vertically built EBM sample (arrow shows the build direction); d) External surface of a horizontally built EBM sample (build direction is perpendicular to the image) $\ldots \ldots \ldots \ldots 168$

C.4 Optical micrographs of SLM produced Ti64 samples. a) Longitudinal cross-section showing columnar grains; b) High magnification longitudinal cross-section image showing fine $\alpha$ martensitic laths (from the boxed region in ' $a$ '); c) Transverse crosssection showing bundles of columnar grains; d) High magnification transverse crosssection image showing fine $\alpha$ martensitic laths in a columnar grain (from the boxed

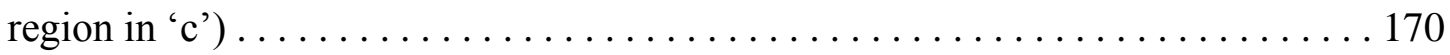

C.5 SEM-SE image of SLM produced Ti64 sample .................... 170 C.6 Optical micrograph of EBM produced Ti64 samples. a) Transverse cross-section;

(b) longitudinal cross-section . . . . . . . . . . . . . . . . . . . . . 171

C.7 SEM-SE image showing Widmanstatten structure in EBM produced Ti64

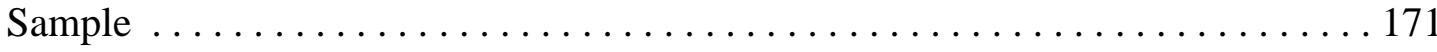

C.8 Optical micrograph of wrought Ti64 (annealed and rolled). a) Transverse crossSection; b) longitudinal cross-section . . . . . . . . . . . . . . . . . . . . 172 C.9 EDS spectrum and the composition in wt\% of Ti64 samples produced by a) SLM 
and b) EBM

C.10 XRD spectrum of Ti64 samples produced by a) SLM and b) EBM . . . . . . . . 174

C.11 Stress-Strain plots of Ti64 samples. a) EBM specimen built in vertical orientation;

b) SLM specimen built in vertical orientation; c) EBM specimen built in horizontal orientation; d) SLM specimen built in horizontal orientation $\ldots \ldots \ldots \ldots \ldots 176$

C.12 $S$ - $N$ curve showing fatigue behavior of Ti64 samples. a) SLM; b) EBM . . . . . . 178

C.13 SEM- SE images of tensile fracture surfaces. a) Overall view of SLM produced Ti64 tensile fracture surface; b) enlarged view from the boxed region in ' $a$ '; c) overall view of EBM produced Ti64 tensile fracture surface; d) enlarged view from the boxed region in ' $c$ ' . . . . . . . . . . . . . . . . . . . 179

C.14 SEM- SE images of fatigue fracture surfaces. a) Overall view of SLM produced Ti64 fracture surface; b) enlarged view from the boxed region in 'a'; c) overall view of EBM produced Ti64 fracture surface; d) enlarged view from the arrow

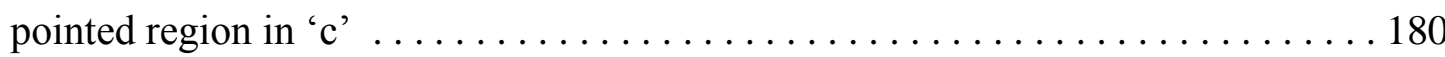




\section{CHAPTER 1}

\section{INTRODUCTION AND LITERATURE REVIEW}

\subsection{Research Motivation and Problem Statement}

Over the past decade, metallic materials have become extensively adopted in solid freeform fabrication processes in forms such as powder and foil. Metal parts and tools can be directly produced using layer by layer joining processes which were previously known as Rapid Prototyping (RP) and now known as Additive Manufacturing (AM) processes. When using metallic powder, Selective Laser Melting (SLM) and Electron Beam Melting (EBM) processes are capable of fabricating complex structures with excellent microstructures which make SLM and EBM not only an improvement over other manufacturing processes, but also innovative material processing technology. This process has aroused further interest in using SLM and EBM methods for fabricating metallic parts for critical components for manufactured systems.

The processes of SLM and EBM are similar to the Selective Laser Sintering (SLS) process developed in the 1980's; but metallic powder is utilized instead of polymers. However, compared to polymers, the high thermal conductivity, propensity to oxidize, high surface tension, and low absorptivity of metal powders make them significantly more difficult to process than polymers (Gibson, 2009). Recent research has shown that density of material fabricated by SLM or EBM is often not 100\% dense (Kumar, 2008a; Facchini, 2009). Porosity is a frequent problem with SLM and EBM parts when characterizing 
process parameters such as energy density, scan speed, hatching strategy, etc. Pores are considered serious defects which deteriorate the quality of SLM and EBM parts.

Engineers who are employing these technologies require more insights into the effect of porosity and defects on mechanical properties. Consistent quality and long-term performance of additively manufactured metallic parts are required to satisfy industrial standards and marketing needs. However, an in-depth understanding of knowledge about defect generation in SLM- and EBM-produced parts have not been sufficient for quality estimation. The morphology and distribution of defects have not been investigated fully when multi-level energy depositions are applied to the powder bed. Effective NonDestructive Testing (NDT) methods to detect defects in AM metallic parts have not been identified for feasibility and practicability. Moreover, how mechanical properties are influenced by the defects has not been clearly studied. Since the defects entrapped in the interlayers could significantly affect the quality and performance of AM parts, a better understanding about the features of defects will help when evaluating their application under various circumstances.

\subsection{Literature Review}

A number of experimental and numerical analyses of Selective Laser Melting and Electron Beam Melting have been published. In this section, those results are summarized and represented in the following aspects. The manufacturing procedure and equipment of SLM and EBM are described. Process capabilities of SLM and EBM are investigated in detail. Controllable processing parameters are identified, and effects of each parameter on performance and properties of fabricated structures are categorized. Melting mechanisms 
during SLM and EBM are listed and summarized. Representative NDT methods are introduced. As one of the most interesting aspects, defects in the SLM and EBM parts are highlighted during the literature review. Existing mechanical properties testing results of SLM or EBM parts are also presented and discussed.

\subsubsection{Additive Manufacturing}

Additive Manufacturing (AM) refers to a "process of joining materials to make objects from 3D model data, usually layer upon layer, as opposed to subtractive manufacturing methodologies." Its synonyms include: additive fabrication, additive processes, additive techniques, additive layer manufacturing, layer manufacturing, and freeform fabrication (ASTM F2792-12). The growth of additive manufacturing in recent years is due to the transformation of these techniques from fabrication of prototypes to rapid tooling (RT) and rapid manufacturing (RM) of end-use components (Campbell et al, 2012).

AM technologies can be further classified to Powder Bed Fusion Processes, Extrusion-Based Processes, Sheet Lamination Processes, Photopolymerization Processes and Printing Processes based upon whether processes use a common type of machine architecture and similar material transformation physics (Gibson et al, 2009 and Kruth et al, 2007). The interest of this study is in two typical Powder Bed Fusion processes: Selective Laser Melting (SLM) and Electron Beam Melting (EBM).

\subsubsection{Selective Laser Melting (SLM)}


Selective Laser Melting (SLM) is capable of building parts directly by selectively melting successive layers of metal powder with a high power laser beam. Similar to the Selective Laser Sintering (SLS) process, SLM utilizes contour information of horizontally sliced cross-sections of a CAD model. Key aspects of SLM include scattering and absorption of laser radiation in the powder, heat conduction, melting and coalescence of metal particles, formation of the melt pool, and its solidification (Gusarov, 2009).

\subsubsection{SLM Process and Commercialization}

SLM processes start by preparing a .STL file which is the de-facto standard file format for additive manufacturing technologies. This file is then loaded into a file preparation software package for slicing the 3D data into layers, usually from $20 \mu \mathrm{m}$ to $100 \mu \mathrm{m}$ in thickness, creating a 2D image of each layer. After that, parameter, values and physical supports are assigned to allow the file to be interpreted and built by different types of additive manufacturing machines.

During the SLM process, atomized fine metal powder has to melt and wet previously solidified layers to avoid balling or warping. A metal substrate plate is usually fastened to the build platform before the first layer of powder is distributed. Once a layer is distributed, laser energy will be applied for contouring and scanning on the powder surface according to the 2D slice of the part geometry using two high frequency scanning mirrors aligned in the $X$-and $Y$-axes respectively, as shown in Figure 1.1. The melted layer will wet and be solidified on the substrate which is designed to lower down one layer thickness along the $Z$-axis. The subsequent layer will be melted and wetted on the previously solidified layer. Over-hanging structures need to be attached with supports to 
the substrate plate to resist residual stress-induced distortion. This process takes place layer by layer inside a chamber under inert gas atmosphere, either Argon or Nitrogen, until the part is completed.

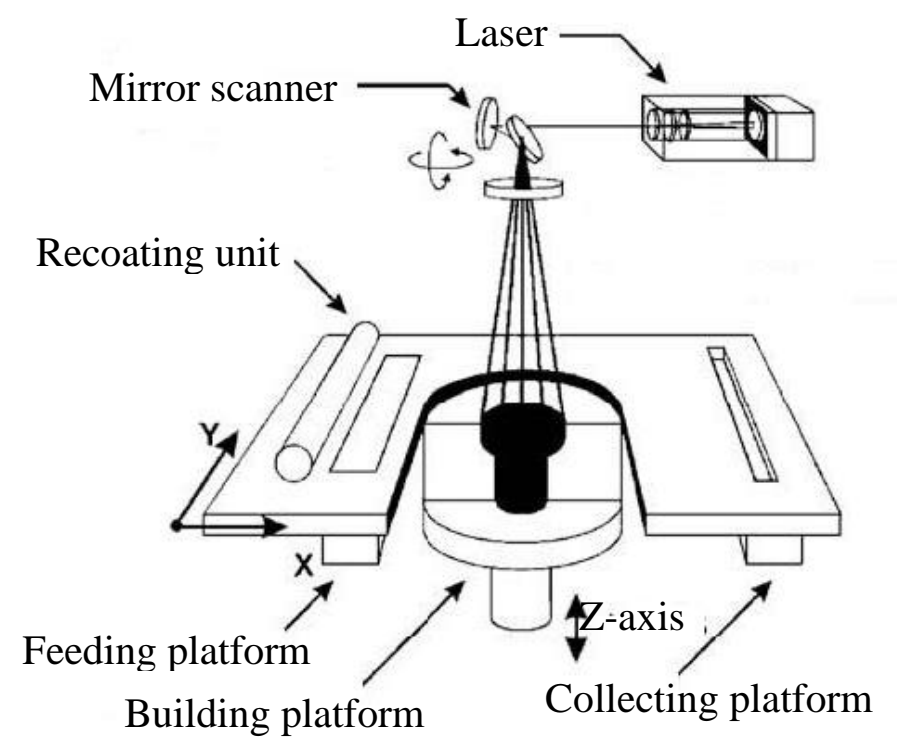

Figure 1.1. Schematic of SLM process (Thijs et al, 2010).

The researchers of Fraunhofer Institute for Laser Technology (Germany) were the first to develop the basic additive manufacturing-based processing techniques for successful laser-based, point-wise melting of metals. Current commercialized SLM machines all emerged from their metal powder melting technique called Selective Laser Powder Remelting (Meiners et al, 1998), which was developed in the mid-1990s. With a decade of progress in process improvement and system development, SLM technologies have been successfully commercialized by many companies. MTT Technologies Ltd was known for producing SLM machines with different platform size configurations and laser powers. But Renishaw Inc. acquired and continued MTT's additive manufacturing business in 2011. EOS GmbH is another SLM machine producer in Germany. The diversity and functionality of EOS machines make it the most successful provider of metal powder 
bed fusion process. Moreover, there are some other companies, such as Concept Laser GmbH, SLM Solutions GmbH, Phenix Systems and Realizer GmbH, which make commercially available laser based systems for direct melting and sintering of metal for injection molds, tooling, dental applications, the MEMS industry and any application where small, complex metal parts are utilized.

\subsubsection{SLM Process Parameters}

Like any new process or method, relevant theoretical, experimental and mechanical testing investigations are necessary to determine the certification and qualification of the SLM technology. Song (1997) reported the process of direct sintering of low-melting metallic bronze powder. The experimental investigations with single spots, lines and layers on the powder bed indicated successful direct sintering of bronze powder without polymer binder or preheating. It was found that process parameters and material features such as laser beam power, scan speed, hatch distance and particle size distribution have an important influence on the melting behavior.

\section{Energy density}

To determine parameters of SLM for high precision is very important but complicated, due to its complex thermo-physical process history. Many process parameters are known to influence the final results individually or together (Kruth et al, 2005a). Kruth proposed "energy density $\left(E_{d}\right)$ " as an important processing characteristic for melting powdered material. This parameter represents the energy supplied by the laser beam to a 
volumetric unit of powdered material and correlates some important laser and scan parameters.

$$
E_{d}=\frac{P_{\text {Laser }}}{V_{\text {Scan }} \bullet S_{\text {Hatch }} \bullet t_{\text {Layer }}}
$$

where $E_{d}$ is energy density, $P_{\text {Laser }}$ is laser power, $V_{\text {Scan }}$ is scan speed, $S_{\text {Hatch }}$ is hatch spacing and $t_{\text {Layer }}$ is layer thickness. From Equation (1.1), it can be seen that the energy density $E_{d}$ is proportional to laser power, but reciprocal to scan speed, hatch spacing and layer thickness.

The ratio of $P_{\text {Laser }} / V_{\text {Scan }}$ is often modified in combination to ascertain the effect of parameters with defined hatch spacing and layer thickness. A process map for continuous laser melting of different types of particles $^{1}$ was determined by Kruth et al (2004), as shown in Figure 1.2. The graphic shows that high scan speeds combined with high laser powers result in a smooth surface and less balling due to rapid solidification of the melt pool. Their results agreed with Simchi et al $(2003,2006)$ who investigated the effect of laser melting iron powder, stainless steel 316L and high-speed steel M2 powder. It was found that the sintered density increases sharply with increasing $P / V$ ratio. Thus, powder densification is proportionally correlated with energy input (Bourell et al, 2002).

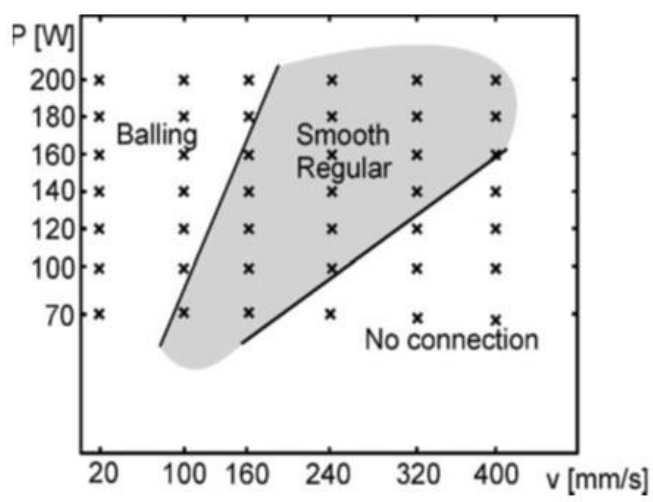

Figure 1.2. Process map for continuous laser melting operation (Kruth et al, 2004).

\footnotetext{
${ }^{1}$ Mixture of $\mathrm{Fe}, \mathrm{Ni}, \mathrm{Cu}$ and $\mathrm{Fe}_{3} \mathrm{P}$ powder
} 
Vandenbroucke et al. (2007) processed Ti-6Al-4V and a cobalt-chromium alloy using a M3 Linear machine (Concept Laser). Their results show that higher energy density leads to higher part density. For low energy input, successive scan tracks are not fully molten and large pores appear along the scan lines, as shown in Figure 1.3. The optimized process parameters for both Ti-6Al-4V and cobalt-chromium were obtained as shown in Table 1.1. Kong et al (2011) also reported an approximately proportional relationship between energy input and thin wall thickness. The energy input per unit length is the control factor for the wall thickness of SLM processed Ti-6Al-4V powder.

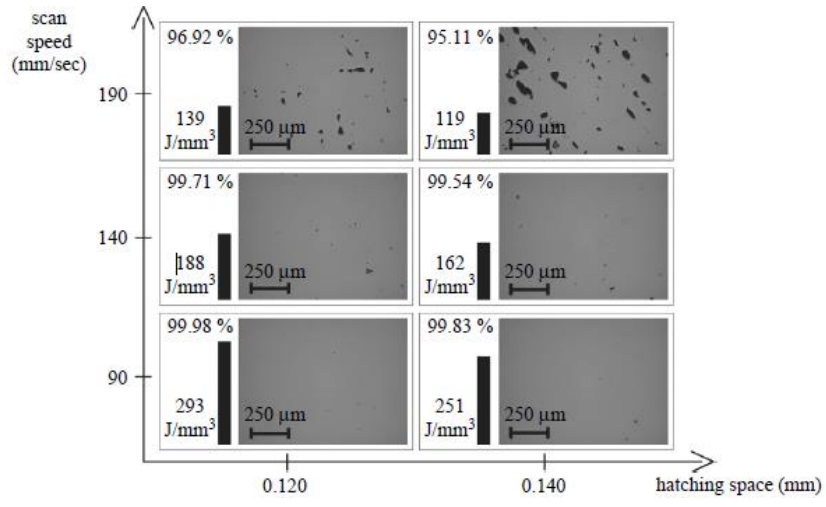

Figure 1.3. Results of parameter study for Ti-6Al-4V (Vandenbroucke et al, 2007).

Table 1.1. Optimized Process Parameters Regarding Part Density and Process Speed for Titanium and Cobalt-Chromium (Vandenbroucke et al, 2007)

\begin{tabular}{lrr}
\hline Material & Ti-6Al-4V & Co-Cr-Mo \\
\hline Melting temperature $\left({ }^{\circ} \mathrm{C}\right)$ & 1650 & 1330 \\
Laser power $(\mathrm{W})$ & 95 & 95 \\
Layer thickness $(\mu \mathrm{m})$ & 30 & 40 \\
Scan speed $(\mathrm{mm} / \mathrm{sec})$ & 125 & 200 \\
Hatch spacing $(\mu \mathrm{m})$ & 130 & 140 \\
Overlap $($ percent $)$ & 35 & 30 \\
Energy density $\left(\mathrm{J} / \mathrm{mm}^{3}\right)$ & 195 & 85 \\
Build rate $\left(\mathrm{cm}^{3} / \mathrm{h}\right)$ & 1.8 & 4.0 \\
Part density $($ percent $)$ & $>99.8$ & $>99.9$ \\
\hline
\end{tabular}


Simply increasing energy density is not always an appropriate route to achieving material densities approaching 100\% (Morgan et al, 2004). Pure titanium powder was sintered by Fischer et al (2003) using a pulsed Nd:YAG laser. They reported that increasing the scan speed leads to a decrease in the surface roughness, while increasing the power results in an increase in deposited energy. Too much deposited power will cause shrinkage and bad surface roughness. Moreover, high pulse repetition rate (frequency) leads to the balling effect. Thijs et al (2010) also provided the optimized parameters for processing of Ti-6Al-4V, but at different values: laser power of $42 \mathrm{~W}$, scanning velocity of $200 \mathrm{~mm} / \mathrm{s}$, hatch spacing of $75 \mu \mathrm{m}$ and layer thickness $30 \mu \mathrm{m}$. This means, compared to the optimized parameters of Vandenbroucke et al, lower energy density is also capable of melting Ti$6 \mathrm{Al}-4 \mathrm{~V}$ powder to high density. Thijs et al indicated that the elongated melt pool is less stable when lowering scan speed from $200 \mathrm{~mm} / \mathrm{s}$ to $100 \mathrm{~mm} / \mathrm{s}$ and $50 \mathrm{~mm} / \mathrm{s}$, because much more energy is input into the powder bed. Correspondingly, sample density is decreased due to the introduction of large pores.

While high energy density can cause the balling effect, lower energy density can also result in a bad surface finish. Morgan et al (2001) conducted experiments with laser melting of fine 316L stainless steel powder with an Nd:YAG laser. They pointed out that balling and curling effects appear serious with power ranges between 25 and $50 \mathrm{~W}$ at scan speeds higher than $300 \mathrm{~mm} / \mathrm{s}$. Solid, dense layers could be fabricated at a higher power $(75 \mathrm{~W})$ and slower scan speed $(<200 \mathrm{~mm} / \mathrm{s})$. They also indicated that hatch spacing is a tradeoff factor for balling, tearing, cohesion and curling. Additionally, lower pulse frequency will cause more vaporization and excessive recoil. Pulse shape is also an effective factor for controlling the energy distribution (Mumtaz et al, 2010). 
In order to acquire a fine melt pool and precise morphology for SLM parts, energy density must be maintained within certain bounds. Otherwise, metallic powder is either hardly melted or balling occurs, which deteriorate the scan tracks. Hauser et al (1999a, 2003) and Akhtar et al (2003) found that stainless steel 314S, M2, and H13 tool steel powders can be fully melted and bonded at certain laser power and scan speed combinations. Yadroitsev et al (2007a, 2007b) analyzed SLM parameters for Inox 904L and Inconel 625 powder (particles size $<20 \mu \mathrm{m}$ ). They found that the greater the value of the $P / V$ ratio, the larger the remelted line. Optimal parameters of power input per unit speed were determined to fall within $P / V=270-420 \mathrm{~W} \cdot \mathrm{s} / \mathrm{m}$, with layer thickness $50 \mu \mathrm{m}$ and hatch spacing $120 \mu \mathrm{m}$.

In addition, scan speed and hatching space also have unique effect on the melting results.

Dutta Majumdar et al (2005) studied laser assisted fabrication of 316L stainless steel and found that the grain size was reduced with increasing scan speed. Micro-porosity was present in the microstructure with increasing scan speed. Childs et al (2005a) found that, at a constant laser power, the variation of scan track mass at different scan speeds is much less than that at a constant absorptivity. This suggests that the thermal history of processing is influential for determining the amount of melt under a laser beam at any instant during processing. Badrossamay et al (2007) defined the thermal history influence as the increase of absorptivity with increasing scan speed. They found heat loss may decrease with scan speed through experimental studies on melting single layers of M2 high speed steel and 316L and 314S-HC stainless steels on the surface of powder beds. 
Simchi et al (2003) proposed that the surface morphology of laser sintered iron was strongly influenced by hatch spacing. Obviously, energy density is varied by varying the hatch spacing. If the hatch spacing is lowered, the scanning vector will cause the melt pool to overlap. However, if the hatch spacing is too large, neighboring melt pools will not touch

each other (Thijs et al, 2010). Yadroitsev et al (2011) also indicated that properties of manufactured parts depend strongly on each single laser-melted track and each single layer, as well as the strength of the connections between them. Analyses of hatch spacing effects on surface morphology show that the hatch spacing should not exceed the average width of the continuous track in order to manufacture a smooth surface.

\section{Scanning strategy}

Density, accuracy and microstructure are always correlated with scanning strategy. In order to obtain customized components with specific mechanical properties, scanning strategy has to be considered before building.

Morgan et al (2002) developed a scanning strategy for direct laser melting stainless steel 316L with over 99\% density. Raster scans were conducted with a gap. Then the second raster scans were carried out on the gaps with about $15 \%$ overlap of the previously scanned layers. To overcome the problem of gas entrapment, the surface of the build layer was smoothed by a third laser process. The consecutive layers were scanned normal to each other in order to eliminate the occurrence of porosity, as shown in Figure 1.4.

Scanning patterns using iron powder were studied by Simchi et al (2003). Powder was scanned along the $X$-axis, $Y$-axis and $X Y$ directions respectively. But there was no obvious difference among these strategies. Kruth et al (2004) also applied SLM to a 
mixture of iron-based particles to investigate deflection in Z-direction. Specimens were scanned with different scanning strategies, as shown in Figure 1.5, and then measured. The results show that scanning along the $X$-direction causes the smallest curvature in that direction, but the largest curvature in the $Y$-direction, and vice versa for the $\mathrm{Y}$ direction. There are no obvious difference in curvature between the large (3), (4) and the small (5), (6) sector strategies. The successive sector scanning (3) and (5) is better than Lease Heat Influence (LHI) scanning (4) and (6), due to its lower thermal gradient.

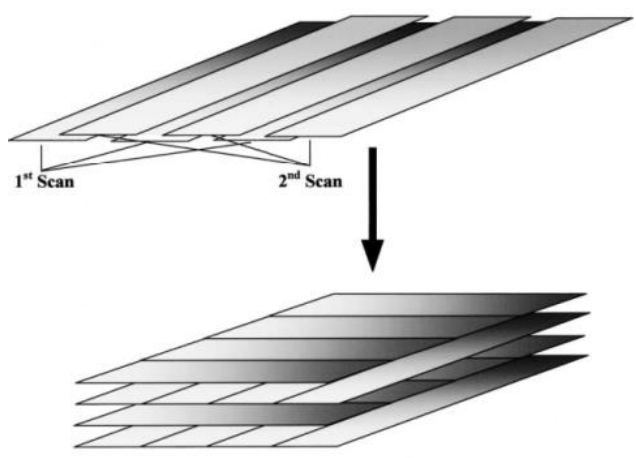

Figure 1.4. Schematic of the scanning strategy investigated by Morgan et al (2002).

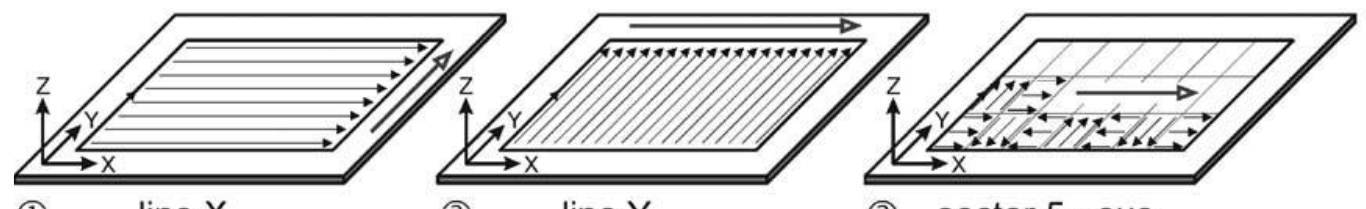

(1) line $X$

(2) line $Y$

(3) sector 5 - suc.

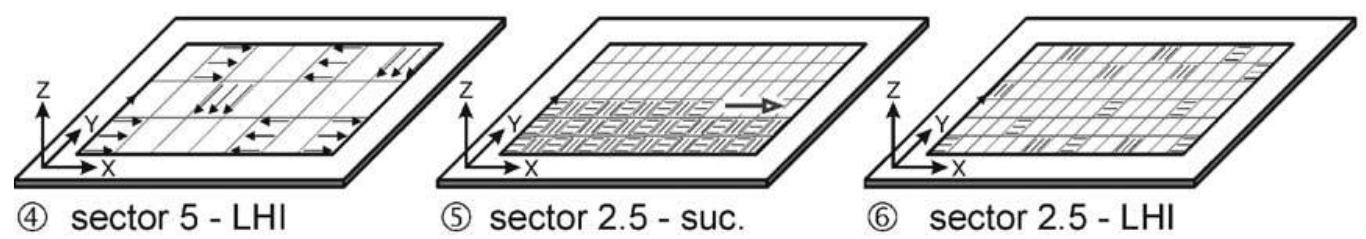

Figure 1.5. The six different scanning strategies compared by Kruth et al (2004).

Building direction has an effect on microstructure evolution which makes the mechanical properties different in various directions. Yadroitsev et al (2009) investigated the influence of scan strategy on the mechanical properties of samples fabricated by SLM 
Inconel 625 powder. It was found that the Young's modulus value of "horizontal" samples is 1.5 times higher than that of "vertical" samples and is close to that of wrought Inconel 625. Microstructure evolution was studied by Thijs et al. (2010) through experiments on Ti-6Al-4V powder with the SLM process. Three different scanning strategies are shown in Figure 1.6. Results show that elongated grains grow along the building direction with heights of the order of $100 \mu \mathrm{m}$ to even several millimeters. The direction of the elongated grains depends on the local heat transfer condition which is determined by the scanning strategy.
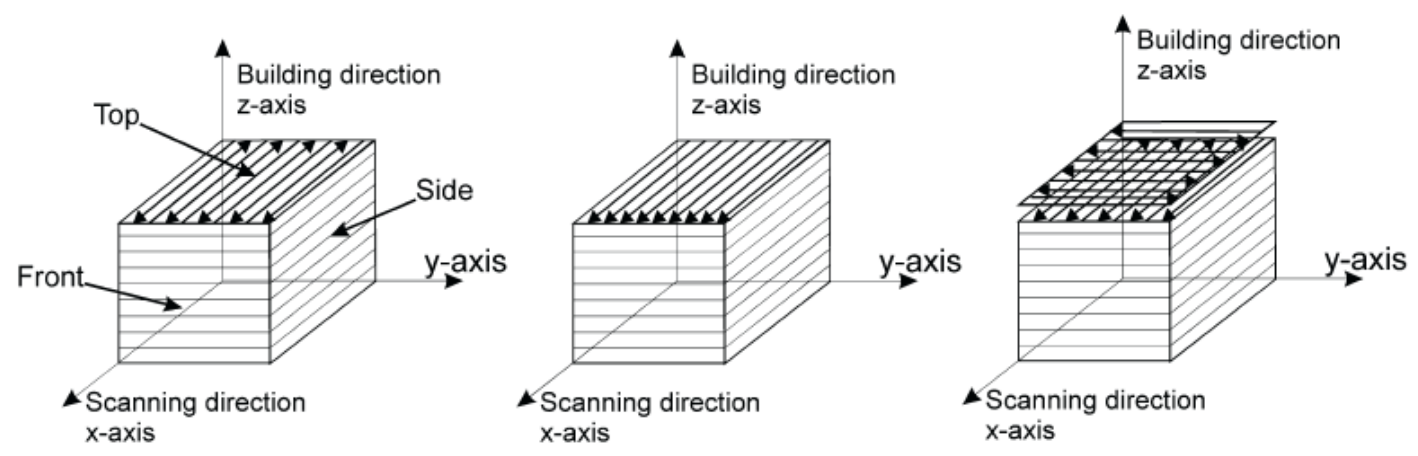

Figure 1.6. Schematic of three scanning strategies (Thijs et al, 2010).

It was noticed by Hauser et al (1999a, 2003) that contour scan power and speed (when performed first) should be different from that of filling, because modifying the contour scan power and speed produced a flattened track at the start of each layer, which can improve the overall layer morphology. Yasa et al (2009) found that contour scanning exaggerates the edge-effect, but, on the other hand, the part's dimensional accuracy requires the borders of the part to be scanned first. Dividing a part into shell and core sections with an overlap between them may not only reduce the edge heights, but also address the contour scanning issue. Moreover, Yasa et al (2011) also found that laser re- 
melting is a promising method to increase the density to almost $100 \%$ and to enhance the surface quality of SLM parts.

\section{Particle size}

Particle size was also considered by some researchers. Niu et al (1999a, 1999b, 2000) performed experiments melting M2 high speed steel powder with different particle sizes. Combinations of laser power and scan speed were varied for M2 gas atomized powder to form balls. It was found that, the larger the average particle size of M2 powder utilized, the higher the minimum energy density for balling. An appropriate particle size range is required to produce smooth, dense surface. Finer powder $(<38 \mu \mathrm{m})$ or coarser powder $(>150 \mu \mathrm{m})$ tends to give less dense structure.

Through laser melting iron-based powder, Kruth et al. (2003) demonstrated the importance of powder composition, powder morphology and processing parameters. They demonstrated that spherical particles, when processed using a pulsed laser and preheating promote higher density. In addition, fine particles were found to fill the voids between larger particles. So powder particle size distribution plays an important role in building dense parts. If SLM parts with higher density are required, not only average particle size but also particle size distribution has to be considered. It was proposed by Spierings et al (2009) that insufficient laser energy could hardly fully melt all particles and thus voids were generated within the scanned layer. However, if the amount of fine particles is enough to fill the voids among large particles or scanned tracks, a higher part density could be reached. Thus, the existence of a certain amount of fine particles can be used to make parts at higher density even if the average particles size of one powder is larger than that of 
another (Hauser et al, 1999b). Moreover, Childs et al (2005b) found that particle size distribution has an effect on thermal conductivity and the size of particles influence penetration of radiation into the bed and the consequent densification.

\subsubsection{SLM Defects}

Pores

Due to the effect of incomplete homologous wetting and balling effects, pores are easily formed during the SLM process. Sercombe et al. (2008) emphasized that material pores are likely to serve as a source of preferential crack nucleation and propagation which will reduce the fatigue life in comparison to $100 \%$ dense material.

Generally, there are two reasons for porosity:

- Usually, high energy input causes a large melt pool which is less stable, resulting in the balling effect. The formed ball is isolated from the rest of the powder by a system of comparatively large pores (Tolochko et al, 2004; Thijs et al, 2010).

- For low energy input, successive scan tracks are not fully molten and large pores appear along the scan lines (Vandenbroucke et al, 2007). The molten pool size is too small to contact its neighboring scan tracks (Li et al, 2010).

\section{Micro cracks}

Large residual stresses are produced by the large solidification undercooling of the melt pool during SLM. As a result, micro cracks can be observed in parts (Facchini et al, 2010). Residual stress of SLM parts was investigated by Shiomi et al (2004b), Mercelis et al (2006) and Casavola et al (2008). It indicates that: 
- The residual stress profile consists of two zones of large tensile stress at the top and bottom of the part, and a large zone of intermediate compressive stress in between.

- The most important parameters determining the magnitude and shape of the residual stress profiles are the material properties, the sample and substrate height, the laser scanning strategy and the heating conditions.

- Heating the substrate, re-scanning each layer or post heat treatment may be effective ways to reduce residual stress

\subsubsection{SLM Part Properties}

The most common applications for SLM are those which require complex geometries \& structures with thin walls and hidden voids or channels. Solid and partially formed or lattice geometries can be produced together to form a single object. Tentative work with SLM for aerospace or medical fields produce functional parts which are hard to fabricate using traditional manufacturing methods such as machining, casting or forging. Therefore, materials for SLM parts need to be investigated to evaluate their mechanical properties for more comprehensive applications in future manufacturing process.

Hollander et al (2006), Vandenbroucke et al (2007), Facchini et al (2010a), Kong et al (2011) and Ramosoeu et al (2011) performed tensile tests on SLM Ti-6Al-4V material. Tensile and yield strengths ranged beyond $1000 \mathrm{MPa}$ which is higher than that of annealed material, although elongation at rupture was slightly lower than for annealed. After an additional post heat treatment, elongation can be increased, while tensile and yield 
strengths were reduced. Kong et al proposed that the tensile results were different when specimens are built along a horizontal versus a vertical direction.

Table 1.2. Results of Mechanical Tests on SLM Samples Compared to Mechanical Properties of Bulk Material from Literature (Vandenbroucke et al, 2007).

\begin{tabular}{llrrr}
\hline & & SLM Ti-6Al-4V & Annealed Ti-6Al-4V & STA Ti-6Al-4V \\
\hline Archimedes & Density $\left(\mathrm{kg} / \mathrm{m}^{3}\right)$ & 4,420 & 4,430 & 4,430 \\
Vickers & Hardness (HV) & 410 (micro) & 350 & 395 \\
\multirow{3}{*}{ Tensile } & 400 (micro) & & \\
& Young's modulus (GPa) & 94 & 110 & 110 \\
& Tensile yield strength (MPa) & 1,125 & 920 & 1,100 \\
& Ultimate tensile strength (MPa) & 1,250 & 1,000 & 1,200 \\
& Elongation at rupture (percent) & 6 & 12 & 10 \\
\multirow{3}{*}{ Bending } & Young's modulus (GPa) & 93 & 110 & 110 \\
& Bending yield strength (MPa) & 1,900 & 1,500 & 1,800 \\
& Ultimate bending strength (MPa) & 2,000 & 1,900 & 2,050 \\
Grindo-Sonic & Young's modulus (GPa) & 101 & 110 & 110 \\
& Shear modulus (GPa) & 38 & 44 & 44 \\
\hline
\end{tabular}

Ramosoeu et al (2011) also conducted hardness tests. A hardness of $344.2 \mathrm{HV}_{10}$ was obtained for the as-sintered samples. A hardness of $304 \mathrm{HV}_{10}$ was obtained for the specimens that were heat treated at $1000^{\circ} \mathrm{C}$ when furnace cooled for 4 hours and $296 \mathrm{HV}_{10}$ when furnace cooled for 34 hours.

Yadroitsev et al. (2007b, 2009) carried out experiments and found that Inconel 625 samples manufactured by SLM have higher values of yield strength and ultimate tensile strength compared with wrought properties. Amato et al (2012) investigated selective laser melting of Inconel 718 pre-alloyed powder in either argon or nitrogen gas. They found asfabricated cylinders oriented in the build direction ( $Z$-axis) and perpendicular to the build direction ( $X$-axis) exhibited columnar grains in the $Z$-direction. The microindentation 
(Vickers) hardness was $3.9 \mathrm{GPa}$ for the as-fabricated materials, 5.7 GPa for the HIP material, and 4.6 GPa for the annealed material. The HIP material also exhibited a columnar phase, while about $50 \%$ of annealed material was recrystallized.

Three point bending tests were done by Kruth et al. (2003) according to the ASTM B312-82 standard to investigate the strength of the mixture of particles. The strength of parts made in a pulsed laser mode proved to be better than those from a continuous mode. The average micro-Vickers hardness was 220 and the Rockwell B hardness was 45. The elastic modulus $E$ was $67 \mathrm{GPa}$. Fretting wear tests were also performed by Kumar (2008a, 2008b) on stainless steel, tool steel, Ti-6Al-4V and Co-Cr-Mo from Concept Laser, EOS and MTT. They indicated that all iron-based SLM materials were found to have higher wear resistance than conventionally produced, milled tool steel.

\subsubsection{Electron Beam Melting (EBM)}

The EBM process was developed at Chalmers University of Technology and commercialized by Arcam AB, Sweden in 2001. It is a versatile process for building porous structures and scaffolds, bio-compatible materials such as titanium alloys and cobalt-base alloys.

\subsubsection{EBM process and commercialization}

Electron Beam Melting (EBM), similarly to SLM, also melts thin layers of distributed powder according to $2 \mathrm{D}$ contour data from a CAD model. But EBM utilizes a high-energy electron beam to fuse the metal powder particles, as shown in Figure 1.7. Moreover, EBM performs the building process in a vacuum chamber because electron 
beams will interact with atoms of gas and can be deflected under an atmospheric condition. The vacuum environment maintains the chemical composition of the material and provides an excellent environment for building parts with reactive materials such as titanium alloys. The main differences between EBM and SLM are summarized in Table 1.3. A substrate plate is also needed for conducting the negative charge induced by the electron beam through to a ground, so that the powder particles don't build up too much electrical charge and repel each other (thus creating a cloud of negatively charged powder particles). Less supports are needed for over-hanging structure in EBM compared to SLM, as the parts being built are held at a higher temperature by heating of the powder bed layer by layer using the electron beam. As a result of less supports, finished parts can be more easily removed from the substrate. Arcam $\mathrm{AB}$ focuses on the development and commercialization of EBM technology. It provides multiple models of EBM machines, such as the Arcam A2 and Q10, for cost efficient production of parts using various materials suitable for use in the medical implants, aerospace and automotive industries.

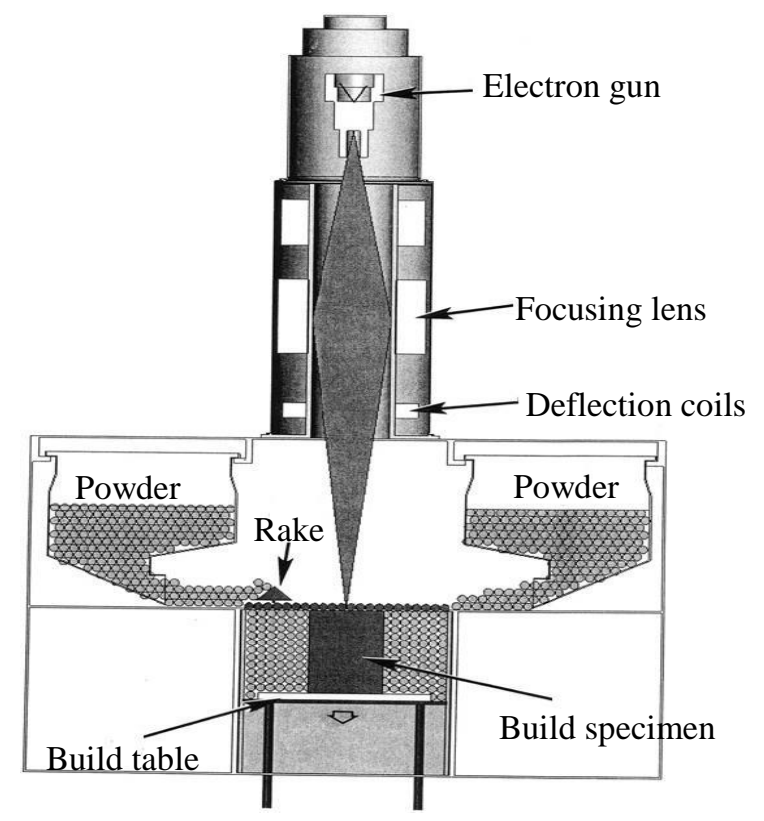

Figure 1.7. Schematic of EBM process (Murr et al, 2009a). 
Table 1.3 Difference between EBM and SLM (Gibson et al, 2009)

\begin{tabular}{l|l|l}
\hline Characteristic & EBM & SLM \\
\hline Thermal source & Electron & Laser \\
\hline Atmosphere & Vacuum & Inert gas \\
\hline Scanning & Deflection coils & Galvanometers \\
\hline Energy absorption & Conductivity-limited & Absorptivity-limited \\
\hline Powder pre-heating & Uses electron beam & Uses infrared heaters \\
\hline Scan speeds & Very fast, magnetically driven & $\begin{array}{l}\text { Limited by galvanometer inertia \& } \\
\text { motor sizing }\end{array}$ \\
\hline Energy cost & Moderate & High \\
\hline Surface finish & Moderate to poor & Excellent to moderate \\
\hline Feature resolution & Moderate & Excellent \\
\hline Materials & Metals (conductors) & Polymers, metals and ceramics \\
\hline
\end{tabular}

\subsubsection{EBM process parameters}

EBM technology is a novel additive manufacturing process. Corresponding research with respect to process parameters is not as prevalent as for SLM. This section presents a brief introduction to energy density, scanning strategy and particle size based on the currently available literature.

\section{Energy density}

The electron beam's high power enables a higher scan rate, a faster build speed and a more even temperature distribution within the part, which gives a fully melted metal with excellent mechanical and physical properties. Energy density can be changed to modify the microstructure of EBM specimens by varying beam current and scan speed. But the energy density mentioned here is not equivalent to energy density in SLM.

Cormier et al. (2004a) investigated processing of H13 tool steel via EBM. Parts were built using a layer thickness of $0.1 \mathrm{~mm}$. Each layer was preheated ten times with a scan speed of $10,000 \mathrm{~mm} / \mathrm{s}$ and a beam current that was increased from $2 \mathrm{~mA}$ on the first 
scan to $20 \mathrm{~mA}$ on the tenth scan. A total of two contours were first melted using a beam speed of $100 \mathrm{~mm} / \mathrm{s}$. The squares were then melted at a speed of $500 \mathrm{~mm} / \mathrm{s}$. The experiments demonstrated that the selection of processing conditions has a significant influence on the build speed and mechanical properties. Microstructural analyses also indicate a small number of isolated shrinkage cracks within certain layers. This was also supported by Cormier et al. (2004b) who indicated that the contour and fill area regions have different microstructures due to the different process parameters used to solidify contours and internal areas.

Fukuda et al (2012) proposed that an increase in the beam current of the incident beam enlarges $\alpha$ grains and increases the relative density, while insufficient energy density caused by beam current may induce large defects. It was concluded that the mechanical properties of EBM Ti-6Al-4V products were very sensitive to the incident beam current and stacking direction, resulting in the exhibition of anisotropic deformation behavior within a limited range of energy density. In addition, the porosity of EBM Ti-6Al-4V products can be controlled by the beam current. The relative density of the specimens increases with an increase in beam current, and reaches up to $99 \%$ after $3 \mathrm{~mA}$.

\section{Scanning strategy}

Lu et al (2009) investigated various scan strategies during the EBM process, including an iterative scan mode, reverse scan mode, randomized block scan mode, and constant length scan mode, as shown in Fig 2.8. Through simulation analyses and experimental melting of Ti-6Al-4V powder, the authors indicated that the reverse scan 
mode can effectively improve the uniformity of the temperature field compared with an iterative scan mode.

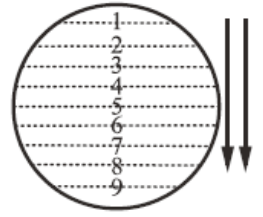

(a) Iterative scan mode

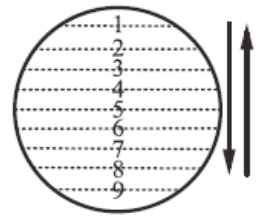

(b) Reverse scan mode

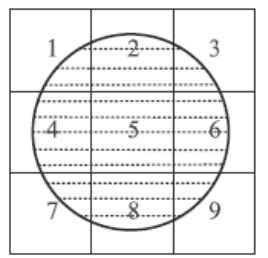

(c) Randomized block scan mode

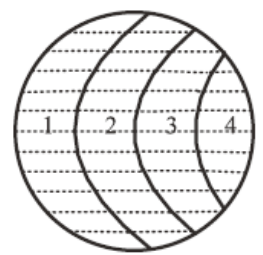

(d) Constant length scan mode

Figure 1.8. Scan strategies of EBM (Lu et al, 2009).

Particle size

No investigations concerning how particle size distribution affects melting results were found during the literature search. A particle size ranging from $30 \mu \mathrm{m}$ to $100 \mu \mathrm{m}$ for Ti-6Al-4V gas atomized powder (Facchini et al, 2009; Murr et al, 2009a; Parthasarathy et

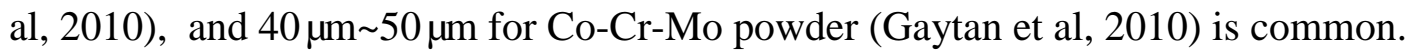

\subsubsection{EBM Defects}

Gaytan et al. (2009) and Murr et al. (2009c) indicated that defects such as porous zones, inclusions and gas bubbles were trapped in the atomized powder particles and retained when electron beam melting Ti-6Al-4V powder. This conclusion meets Biamino's discussion which proposed that two kinds of pores could be found when electron beam melting Ti-48Al-2Cr-2Nb powders. Biamino et al. (2011) indicated that bigger elongated pores were attributed to hatch spacing in the EBM process since these pores were located between two subsequent layers and were elongated exactly in the layer direction. These pores could be completely eliminated by process optimization. Smaller spherical pores 
were due to defects already present in the powder particles caused by gaseous argon entrapped during the atomization process.

\subsubsection{EBM Part Properties}

Tool Steel

H13 tool steel powder was investigated by Cormier et al (2003, 2004a) using the EBM process. The hardness of both air-cooled and annealed specimens was measured. The air-cooled specimens had hardness values ranging from 48.0 to $50.0 \mathrm{HRC}$ while the annealed specimens had HRC values under 20.

\section{Co-Cr-Mo Alloy}

Kircher et al. (2009) conducted investigations on the chemical, microstructural and mechanical properties of several test specimens produced by EBM of a Co-Cr-Mo alloy before and after heat treatment. They found that heat treatments result in re-dissolving a portion of the carbides and increasing the grain size of the material. As-built specimens have tensile properties of: yield stress $717 \mathrm{MPa}$ and UTS $1110 \mathrm{MPa}$ in the horizontal direction, and yield stress $786 \mathrm{MPa}$ and UTS $869 \mathrm{MPa}$ in the vertical direction. Gaytan et al. (2010) also examined and compared the microstructures and mechanical behavior of parts by EBM of Co-Cr-Mo powder. They found that different types of EBM Co-Cr-Mo structures had different mechanical properties. The corresponding hardness for the mesh struts varied from 5.6 to $6.8 \mathrm{GPa}$ in contrast to solid monolithic components which varied from 4.4 to $5.9 \mathrm{GPa}$. While a slight increase in carbide content for the reticulated mesh structures accounts in large part for this hardness increase, a more rapid cooling rate 
especially for the reticulated mesh was also a contributing factor. The tensile testing of specimens, machined from cylindrical components fabricated by EBM, produced average UTS of $1.45 \mathrm{GPa}$, yield stress $0.51 \mathrm{GPa}$, and elongation of $3.6 \%$.

\section{Ti-6Al-4V Alloy}

Facchini et al. (2009) investigated the microstructural and mechanical properties of EBM Ti-6Al-4V and the stability of the as-built microstructure upon heat treatment. They showed that the microstructure has a very fine and acicular morphology, because of the intrinsically high-solidification rate of the process. The yield stress ( $830 \pm 5 \mathrm{MPa})$ and UTS (915 $\pm 10 \mathrm{MPa}$ ) of as-built EBM specimens (surface machined after production for better finishing) are higher than for wrought and annealed material, and comparable to HIPed EBM material. However, elongations at break are lower. The Vickers hardness of the asbuilt EBM Ti-6Al-4V alloy was 3.21 GPa, while microhardness was 3.51 GPa. HIPed EBM hardness values are lower than as-built ones. Fatigue tests carried out on six plane bending machines show that fatigue resistance of the as-built alloy is lower than that of the wrought and comparable with that of the as-cast materials. These results about acicular $\alpha$ and associated $\beta$ microstructures agreed with the investigation of Murr et al. (2009a, 2009b, 2009c). However, tensile and hardness tests presented slightly higher values which reached to $1180 \mathrm{MPa}$ and at least $3.6 \mathrm{GPa}$ respectively.

A comparison between EBM, SLM and hot worked Ti-6Al-4V was performed for mechanical properties (Facchini et al, 2010a; Koike et al, 2011). From Figure 1.9, they found that yield stress, UTS and elongation rate are very different from each other. These differences could be explained by the investigation of Sieniawski et al (1997) and Filip et 
al (2003) with respect to the morphology of the lamellar microstructure and phase composition. They pointed out that the cooling rate from the $\beta$-phase range and ageing conditions have an effect on the microstructure, volume fraction and chemical composition of the $\beta$-phase, which leads to significant effects on mechanical properties and cracking during fatigue. Kubiak et al (1998) and Malinov et al (2002) analyzed the effect of cooling rate and heat treatment on titanium alloys. They found continuous cooling of titanium alloys from the $\beta$-range produces a microstructure in the shape of colonies of parallel $\alpha$ phase lamellae in primary $\beta$-phase grains with various phase compositions $\left(\alpha+\alpha^{\prime}\left(\alpha^{\prime \prime}\right)\right.$, $\left.\alpha+\alpha^{\prime}\left(\alpha^{\prime \prime}\right)+\beta, \alpha+\beta, \alpha+\beta+\mathrm{TiCr}_{2}\right)$. In order to gives rise to competitive microstructures of titanium, proper tempering or ageing processes could be adopted to transform $\alpha^{\prime}$ phases to $\alpha+\beta$ phases, as was proposed by Gil Mur et al (1996) and Ivasishin et al (1999). However, SLM and EBM are capable of producing new and unusual, even non-equilibrium microstructures which extend the contemporary materials science and engineering paradigm relating to structure-properties-processing-performance relationships (Murr et al, 2012).

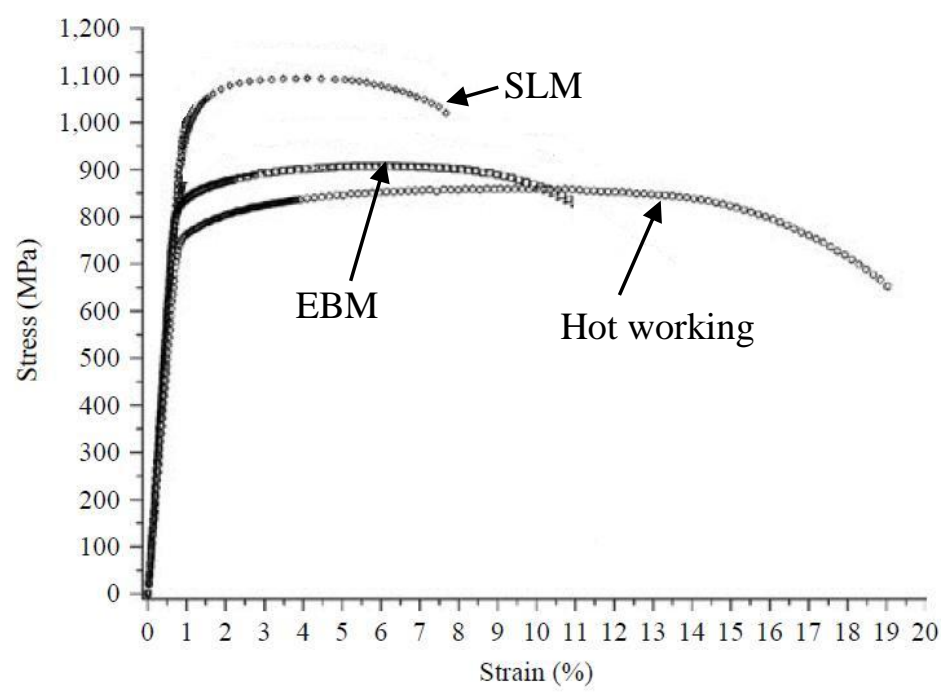

Figure 1.9. Stress-strain curves for Ti-6Al-4V (Facchini et al, 2010). 
Inconel Alloy

Murr et al (2011) studied the microstructures and microstructural architecture as well as mechanical behavior of as-built and HIPed Inconel 625 components produced by EBM prealloyed powders. They found that the microstructure of as-built and HIPed components exhibited contrasting mechanical properties. The as-built tensile cylinders had a yield stress of $410 \mathrm{MPa}$ and a UTS of $750 \mathrm{MPa}$ in contrast to 450 and $890 \mathrm{MPa}$ for annealed, wrought Inconel 625. The corresponding elongation is comparable to annealed, wrought alloy 625 at $44 \%$. The corresponding tensile data for the hipped EBM tensile cylinders exhibited a yield stress of $330 \mathrm{MPa}$, a UTS of $770 \mathrm{MPa}$, and an elongation of $69 \%$.

Bronze

Ramirez et al (2011a, 2011b) proposed to fabricate $\mathrm{Cu}$ components by EBM from atomized $\mathrm{Cu}$ powder which contains a high density of $\mathrm{Cu}_{2} \mathrm{O}$. Microstructure indicates precipitate-dislocation architecture which has very similar spatial and geometrical arrays to the columnar carbide arrays created in a Co-base, carbon-containing alloy by EBM: celllike, equiaxed arrays in the horizontal reference plane perpendicular to the build direction. The hardness for these architectures ranged from HV 83 to 88 , in contrast to the original $\mathrm{Cu}$ powder micro hardness of HV 72 and the commercial $\mathrm{Cu}$ base plate hardness of HV 57.

\subsubsection{Melting Mechanism of Metallic Powder}

Solid-state sintering, chemically-induced binding, liquid-phase sintering, and full melting are four different fusion mechanisms in powder bed fusion processes (Kruth et al, 
2005b). Selective laser melting and electron beam melting typically utilize the full melting mechanism which completely melts all particles of metal powder and generates a compact and stable solid body. Powder material is subjected to a series of complicated chemical and physical phenomena such as oxidation, wetting, epitaxial solidification and vaporization. Thermal energy from subsequent scans of a laser or electron beam is typically sufficient to re-melt a portion of the previously solidified solid structure (Das, 2001a, 2001b, 2001c, 2003; Gusarov et al, 2009; Verhaeghe et al, 2009). This type of full melting is very effective for creating well-bonded, high-density structures from engineering metals.

\subsubsection{Defect Detection via Non-destructive Testing (NDT)}

Internal defects of SLM and EBM component can be examined by destructive characterization techniques such as cutting, milling or polishing so as to expose them for viewing under optical or electron microscopy. But destructive methods usually take significant sample preparation time and only present two-dimensional information such as cross-section and location. If three-dimensional information such as shape and contour is needed, destructive methods are not ideal for meeting these kinds of requirements. Thus, non-destructive testing methods are proposed to study defects in SLM and EBM samples in this study.

\subsubsection{Introduction to NDT}

NDT technicians and engineers define and implement tests that locate and characterize material conditions and flaws that might otherwise cause planes to crash, reactors to fail, trains to derail, pipelines to burst, and a variety of less visible, but equally 
troubling events. These tests are performed in a manner that does not affect the future usefulness of the object or material. In other words, NDT allows parts and material to be inspected and measured without damaging them (Xu, 2008).

\subsubsection{Representative NDT methods}

NDT is divided into various methods based on a particular scientific principle. The most common NDT methods are Visual and Optical Testing (VT), Radiography (RT), Magnetic Particle Testing (MT), Ultrasonic Testing (UT), Penetrant Testing (PT), Electromagnetic Testing (ET) and, Thermography Testing (TT). Each method may be further subdivided into various techniques. But not all NDT methods are applicable to detect internal defects in SLM or EBM components. For example, VT, MT and PT methods are usually adopted for detecting superficial defects or cracks, which make them useless for discovering internal defects of SLM or EBM parts. ET (such as eddy current testing) requires the surface of a part be accessible to the probe, which limits its application to complex SLM or EBM parts. This section briefly introduces NDT methods and focuses on the TT, UT and RT method.

\section{Thermography Testing (TT)}

In thermographic non-destructive testing and inspection, infrared cameras are used to observe how heat propagates in materials as the material is heating up or cooling down. Internal defects within the inspected material strongly affect the diffusion of heat. Thus, defective areas may look cooler or hotter compared to regular areas of the sample. This temperature difference caused by regular and defective material is visible through infrared 
cameras. A wide variety of energy sources are available to excite the temperature difference between defective areas and regular areas. The most common types can be divided into optical, mechanical or inductive. Figure 1.10 shows a typical example of an optical heat source thermography inspection. Mechanical thermal excitation is also known as vibrothermography, where energy is applied into the specimen by means of mechanical oscillations using a sonic or ultrasonic transducer in contact with the specimen. Inductive excitation can be applied internally to electro-conductive-materials, through generating eddy currents, thus heating up the specimen and the internal defects (Hung et al, 2009).

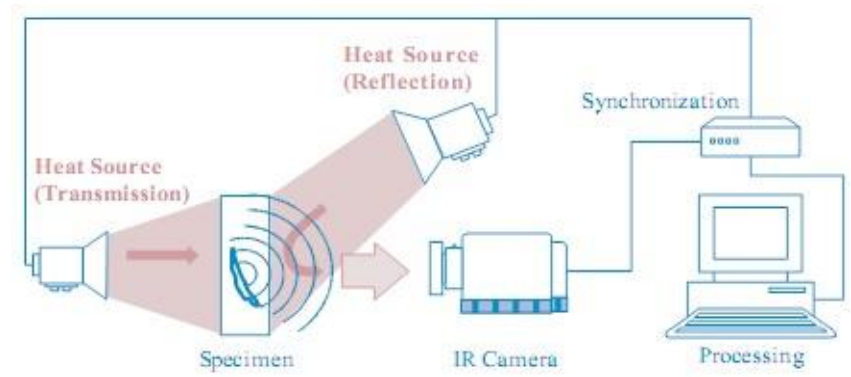

Figure 1.10. Experimental setup for thermography inspection (www.visiooimage.com).

\section{Ultrasonic Testing (UT)}

In ultrasonic testing, high-frequency ultrasound is transmitted into a material to measure the depth of a defect or locate changes in material properties. Ultrasonic vibrations are generated by applying high frequency electrical pulses to a transducer. Ultrasonic sound is introduced into a test object and reflected from internal imperfections or the part's geometrical surfaces to a receiver, as shown in Figure 1.11. Generally, a coupling medium such as oil is applied between the search unit \& the test part. Ultrasonic testing is often performed on metals or alloys in industries including aerospace, automotive and other transportation sectors. The austenitic stainless steel welds of primary coolant piping 
systems are commonly checked using ultrasonic techniques to locate and size potential defects (Chassignole et al, 2010).

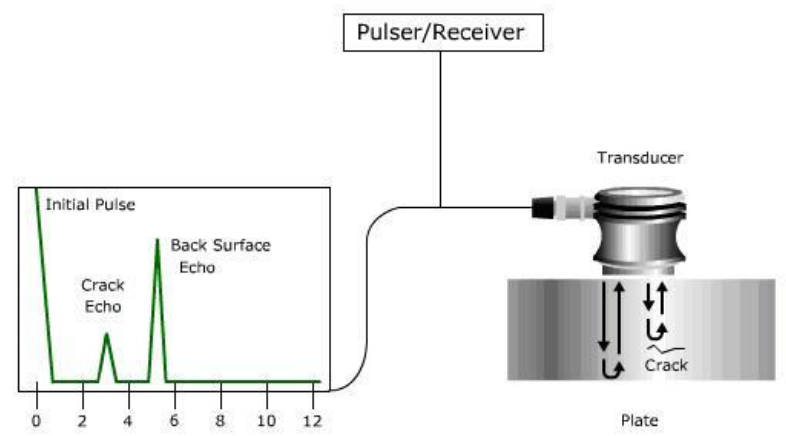

Figure 1.11. Schematic of ultrasonic testing (www.virtualengg.com).

\section{Radiography Testing (RT)}

RT involves using the penetrating abilities of electromagnetic radiation such as gamma-radiation or X-rays to examine the internal defects of objects (Carvalho et al, 2008). A radioactive source is used to send radiation through a part. Material discontinuities such as voids or changes in contour affect the degree of radiation absorption. Moreover, specimens will have differences in density, thickness, shapes size, or absorption characteristics, thus absorbing different amounts of radiation. The unabsorbed radiation that passes through the part is recorded on film, screens, or other digital media. Indications of internal characteristic will be exposed as voids, pores or cracks, as shown in Figure 1.12.

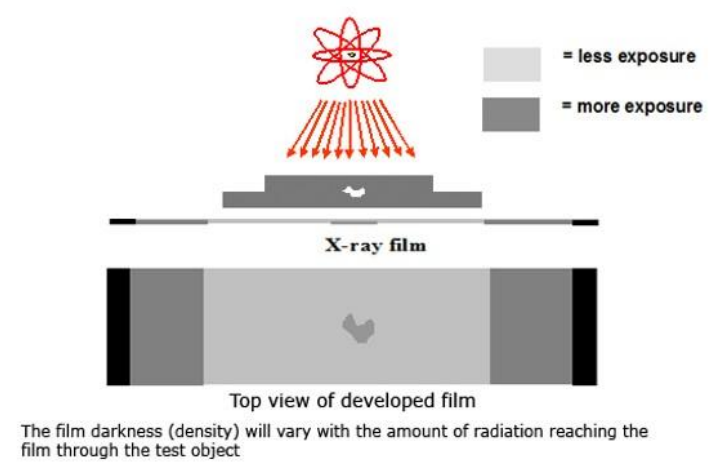

Figure 1.12. Schematic of radiography testing (www.energyworkforce.net). 
The intensity of the gamma-radiation or X-ray is modified according to the internal structure of the specimen to generate a radiograph. The radiograph is interpreted for the flaws present in the specimen. The radiography testing method is used for the detection of internal flaws on a wide variety of products such as forgings, castings and weldments. Parthasarathy et al (2010) conducted micro-CT scan analyses of samples of well-formed titanium struts made by EBM with porosity from $50 \%$ to $70 \%$.

\subsubsection{Effect of Defects on Mechanical Properties}

Porosity is typically detrimental to mechanical properties. Pores act as stress concentrators leading to an earlier onset of plasticity and localization of strain (Yadroitsev et al, 2009). However, only a few investigations into SLM and EBM were focused on the effect of defects on mechanical properties. This section briefly introduces the results of these investigations into stiffness, bending strength, fatigue and hardness.

\section{Stiffness}

Ramirez et al (2011a) and Murr et al (2010) studied cellular structures (reticulated meshes) and stochastic foams fabricated by EBM from precursor $\mathrm{Cu}$ and Ti-6Al-4V powder. Although these structures are designed with regular pores, they could be considered EBM specimens with built-in defects. The porosity or density can be seen as a representation of the defect distribution rate. They proposed that the stiffness or Young's moduli for these cellular structures vary with density and vary inversely with porosity, especially for open cellular structure foams 


\section{Bending strength}

Three point bending tests were done to determine the bending strength of parts made by SLM of iron-based powder (Kruth et al, 2004), as shown in Figure 1.13. Three main laser regimes were tested: continuous mode, pulsed mode and pulsed mode with the recoil effect. It can be found that the bending strength increased with the material density. This means that internal defects have a negative effect on bending strength.

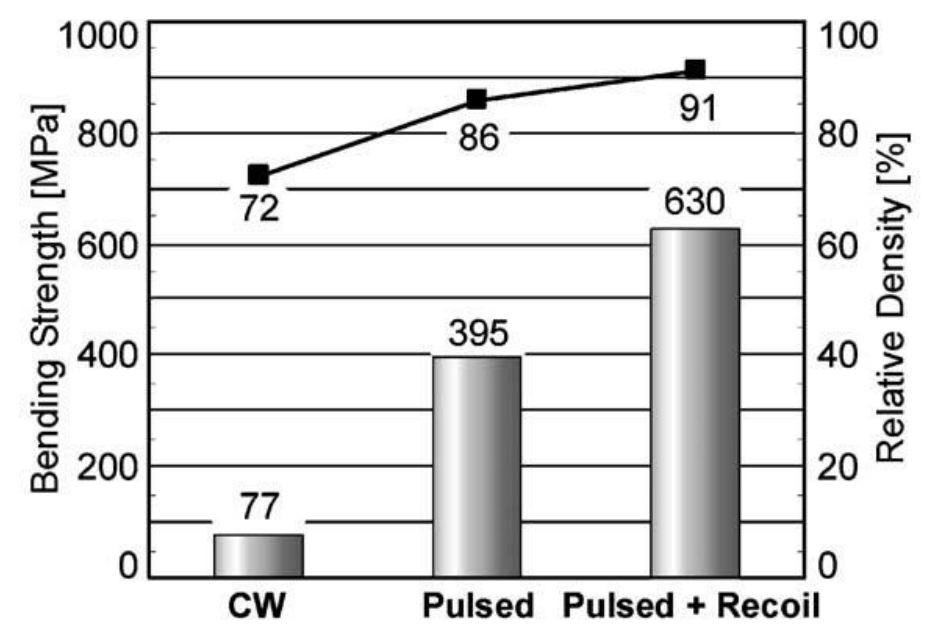

Figure 1.13. Bending strength and relative density for different laser regimes.

\section{Fatigue}

Santos et al (2002) conducted laser melting of pure titanium powder and fabricated specimens with densities higher than 95\%. Although the tensile strength tests showed results comparable to the wrought material, the impact and torsional fatigue strengths are low because of porosity. Li et al (2012) carried out compression fatigue behavior tests on Ti-6Al-4V mesh arrays with high porosities of $60-85 \%$. It was found that the fatigue strength increases with increasing relative density. This indicates that certain defects deteriorate EBM specimens' ability to resist cyclic stresses. 


\section{Hardness}

Through measuring the hardness of SLM Ti-6Al-4V samples, Vandenbroucke et al (2007) proposed that micro hardness does not vary significantly for samples with certain porosity, because micro hardness was generally measured at pore-free regions. Since, macro hardness is more dependent on porosity, the measured values were lower than those for micro hardness and energy density had a larger influence, as shown in Figure 1.14.

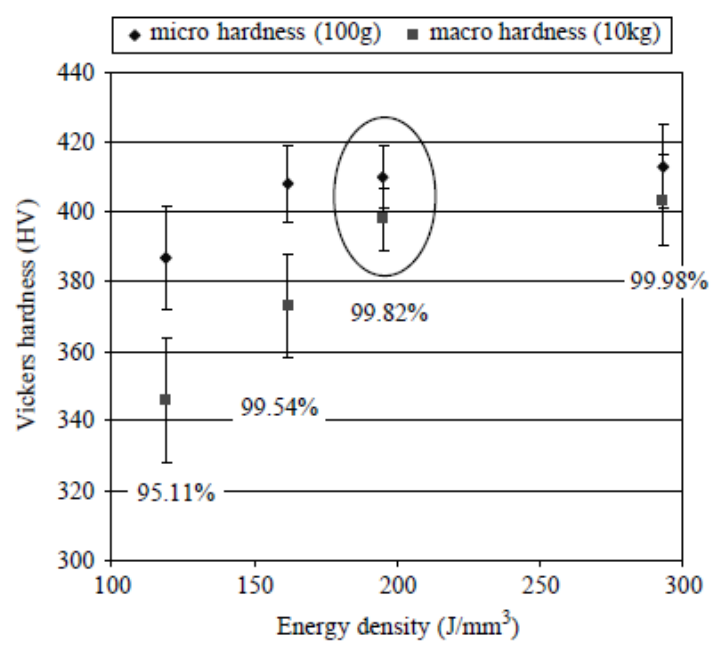

Figure 1.14. Results of hardness analysis for Ti-6Al-4V (Vandenbroucke et al, 2007).

\subsubsection{Conclusions from Literature Review}

The literature reviewed here relating to SLM and EBM show that knowledge of the effects of defects on properties is not complete. The SLM process has been investigated for a longer time than for EBM, thus, more literature are available for interpreting how process parameters influence the melting, microstructures and part performance of components. Since the EBM process is a more recently developed technology (from the beginning of this century) less is known about electron beam control and process parameter effects. However, as they both involve melting of powder layers, the process parameter effects for both are similar. In general, energy density plays an important role on the surface 
morphology and microstructure. Laser or electron power, scan speed and hatch spacing are all related to energy density. Moreover, scanning strategy and particle size distribution also affect the melting results of various types of powder. The ratio of $P_{\text {laser }} / V_{\text {scan }}$ is considered a significant influence on the porosity of SLM parts. Particle characteristics of the powder are also regarded as a vital factor influencing pores in EBM.

Defects caused by certain process parameters or particle characteristic have their own features inside of SLM or EBM parts. Balling effects and residual stress usually result in pores or micro cracks in the internal area of SLM parts, while gas trapped in the EBM particles likely generates pores between successive layers of EBM parts. Thus, the cause of the defect morphology can be discriminated.

It is convenient to employ non-destructive testing methods to explore defects without destroying specimens. However, no work was found to provide applicable NDT methods for detecting defects in SLM or EBM parts. From the various NDT technologies introduced, it was found that Thermography Testing (TT), Ultrasonic Testing (UT), and Radiography (RT) are the mostly likely proper candidates for internal defect detection, because no direct contact is need for the probe to the surface of SLM or EBM parts.

Many common materials such as stainless steel, tool steel, titanium, Inconel and Co-based alloys were tested after being fabricated by SLM or EBM processes. Some material properties of as-built parts are totally different from that of wrought or hot worked products; some even superior to regular materials. It was concluded that the melting and solidification involved in SLM and EBM causes the microstructure of parts made that way to be different from those made using traditional techniques. Microstructural 
characterization has helped explain the difference between the mechanical properties of SLM and EBM and those made by traditional processes.

Porosity typically has a negative impact on mechanical properties such as mechanical strength, fatigue performance and hardness. Few published studies have analyzed the effect of defects or statistically investigated correlations between porosity and mechanical properties. In addition, little research has been carried out on how mechanical properties are influenced by the morphology of defects.

\subsection{Research Goal and Objectives}

In order to be able to make use of the speed and geometric flexibility of SLM and EBM processes, a more careful investigation of the quality and expected service life of components made this way must be carried out to give designers the data they need to employ these processes correctly. A component's quality and service life are always associated with the identification, characterization and effects of defects. Thus, this research goal is to establish a fundamental understanding of defects in parts made by the SLM and EBM processes.

From an overview of the available literature, it was determined that no specific research has been conducted to quantitatively correlate defect generation to process parameters, no work was found to provide insight into applicable NDT methods for detecting defects in SLM or EBM parts; and little research has been carried out on mechanical properties of parts with various categories of defects. Thus, the objectives of the current research are to study how process conditions control defect generation, how to 
detect and characterize these defects, and how defects affect mechanical properties of components created from metallic powder by SLM and EBM processes.

In order to better understand the categorization of defects, key concepts must be defined before stating the specific research objectives, namely:

- Stochastic defect- a defect which is generated randomly due to a balling effect, local irregularity of molten powder, or error in one or more of the machine's operating parameters.

- Deterministic defect- a defect which is designed in a CAD model and fabricated with certain dimensions and geometry under optimized processing parameters (such a defect could also occur, for instance, due to an error in a CAD model or in its conversion during preparation for a build)

- Marginal parameter- a process parameter combination in SLM or EBM which result in stochastic defects.

According to the research goal and objectives, specific tasks are identified and summarized as follows.

(i) The study begins by generating stochastic defects using designed experiments to define marginal processing parameters. Ti-6Al-4V powder is utilized as the primary material. Previously identified optimized processing parameters are used as a starting place around which to design the experiments and to correlate parameters with porosity.

(ii) Defect morphology and distribution are then correlated to marginal parameters through optical microscopy by destructive techniques. Relationships between processing parameters and stochastic defects are identified for SLM/EBM 
parts based on image processing results. An analysis of the morphology and dimensions of stochastic defects is conducted.

(iii) Specimens with defects are investigated using representative Non-Destructive Testing (NDT) methods to characterize the effectiveness of each method. The most effective NDT method is used to evaluate the defect dimension and porosity to match the actual defective situation of SLM/EBM parts.

(iv) Mechanical property tests are conducted to determine the practical applicability and the working quality that can be expected when stochastic defects are present. Tensile tests and fatigue tests are the main feedback mechanism to confirm the effect on SLM/EBM parts containing stochastic defects. Effect of defect morphology and distribution rate is identified and correlated to tensile and fatigue results. A series of enriched data sets and graphs of tensile strength and fatigue testing results are obtained.

\subsection{Dissertation Format}

This dissertation is prepared using a multiple-paper format. Four papers are composed to describe the research results, discussions and conclusions based on the research goals and objectives mentioned from the previous section.

Chapter 2 is a paper published in the Solid Freeform Fabrication Symposium (2013). It explores the correlation between marginal parameters and porosity in SLM- and EBMproduced parts.

Chapter 3 is also a paper published in the Solid Freeform Fabrication Symposium (2013). Image processing was adopted for statistically analyzing the characteristics of 
defects, such as distribution of defect area and dimensional proportion of stochastic defects in SLM- and EBM-produced parts.

Chapter 4 is prepared as a journal paper for NDT \& E International. Micro-CT was utilized to evaluate stochastic defects in specimens fabricated by SLM and EBM. Porosity of the specimens is also estimated based on the CT slices and reconstructed 3D models.

Chapter 5 is a paper prepared for Rapid Prototyping Journal. It investigates the influence of defects on mechanical properties (tensile and fatigue strength) of SLM- and EBM-produced Ti-6Al-4V specimens. Fractography investigation was performed to compare crack initiation and propagation at different porosity.

Chapter 6 discusses major conclusions from this work and identified future work. Related materials are contained in Appendices. 


\section{CHAPTER 2}

\section{THE EFFECTS OF PROCESSING PARAMETERS ON DEFECT REGULARITY IN TI-6AL-4V PARTS FABRICATED BY SLM AND EBM ${ }^{2}$}

\subsection{Introduction}

Metal powder bed fusion based Additive Manufacturing (AM) processes, such as Selective Laser Melting (SLM) and Electron Beam Melting (EBM), are successfully being used to manufacture parts from metallic materials including stainless steel, maraging steel, cobalt chrome, titanium alloys and more. In particular, additively manufactured Ti-6Al-4V parts are of wide interest for aerospace, biomedical and industrial fields due to its fracture resistance, fatigue behavior, corrosion resistance and biocompatibility (Soboyejo et al, 2006).

An SLM machine selectively melts metallic powder layers, forming a solid part on a building plate. Inside an SLM build chamber, there is a material dispenser platform along with a recoating unit used to feed new powder over the build platform, as shown in Figure 2.1. Parts are then built up additively, layer by layer, based upon a sliced CAD representation of the desired part.

\footnotetext{
2 This chapter is a paper published in the Solid Freeform Fabrication Symposium (2013). All permissions to use this paper as a part of this dissertation are contained in Appendix D.
} 


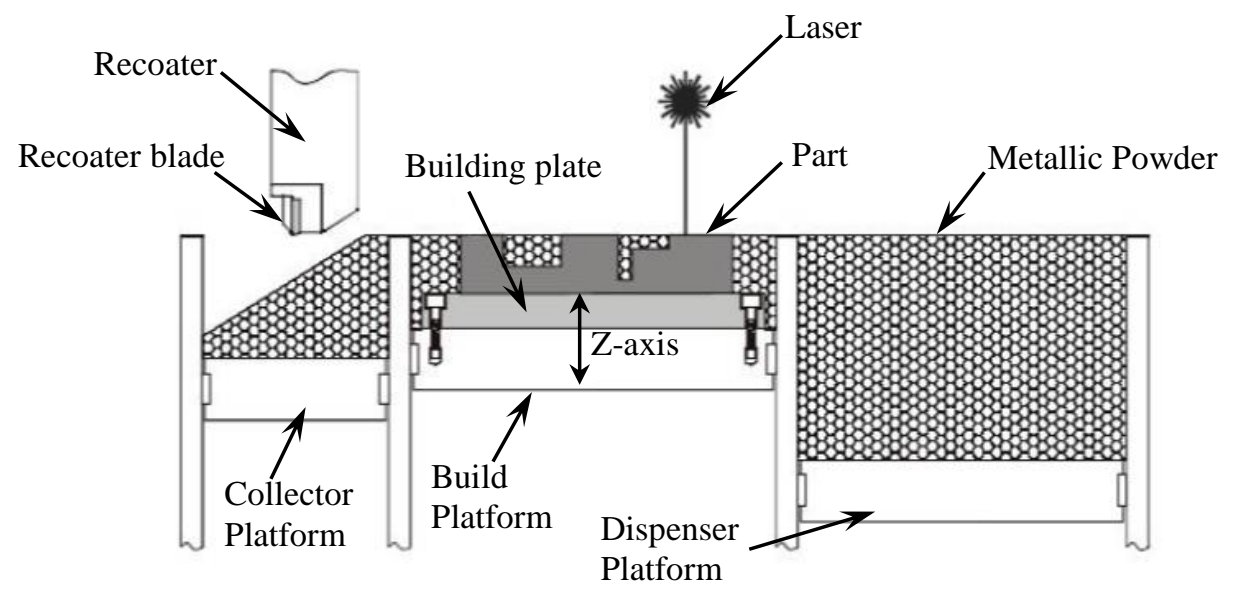

Figure 2.1. Schematic of SLM process (source: EOS basic training manual).

In the EBM process, instead of a laser beam, an electron beam is generated via a tungsten filament in an electron gun, as shown in Figure 2.2. The powder bed is first preheated using a defocused electron beam in order to enhance the conductivity and cohesion of particles, and to avoid powder spreading in the chamber.

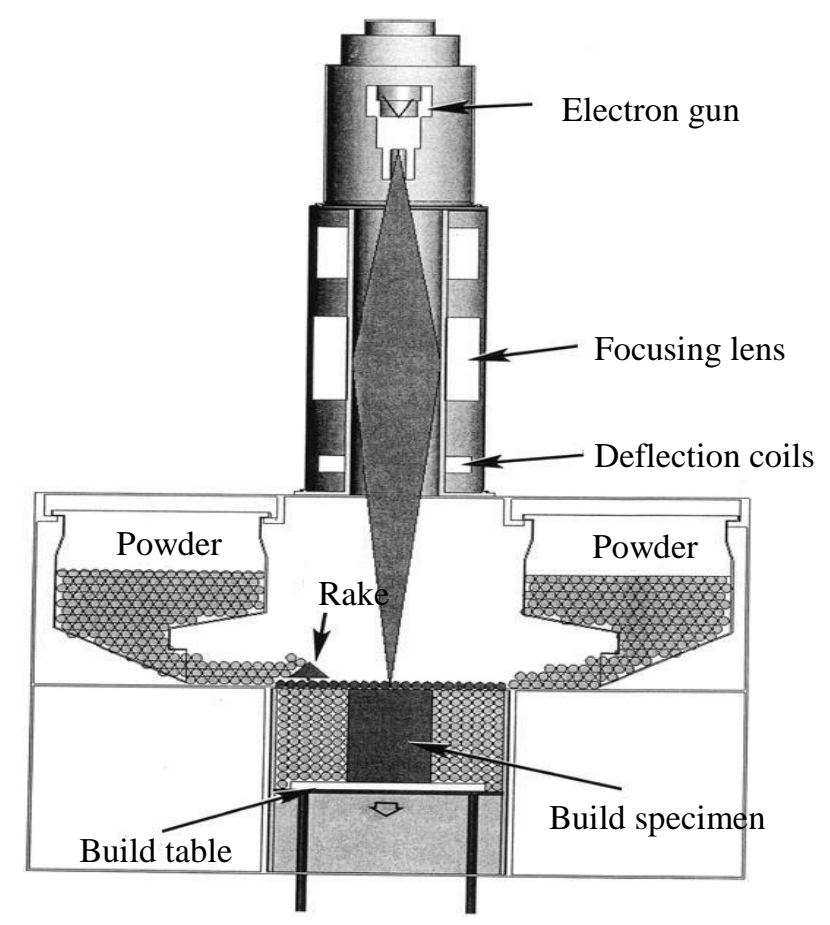

Figure 2.2. Schematic of EBM process (Murr et al, 2009a). 
The microstructural characteristics of EBM-built material are also influenced by the pre-heating process. A more concentrated beam of electrons is then used to melt the metal powder according to the proscribed CAD data. When the electrons penetrate the powder surface and into the powder grains, their velocity is slowed. By doing this, their kinetic energy is converted into thermal energy and the metal powder reaches its melting temperature (Hiemenz, 2007). Electromagnetic lenses are used to control the electron beam, in contrast to the scanning galvanometers used in SLM.

In recent years, SLM and EBM of Ti-6Al-4V have made significant progress (Levy et al, 2003; Vandenbroucke et al, 2007; Murr et al, 2012; Facchini et al, 2009), especially related to optimization of processing parameters and characterization of Ti-6Al-4V materials. However, many authors indicated that SLM and EBM-built materials are subject to porosity inclusion, which influences mechanical properties (Sercombe et al, 2008; Biamino et al, 2011). In order to explain defect generation, this study carried out experiments with various processing parameter combinations. For clarity, a number of nonoptimal parameter combinations were named "marginal parameters". The effect of marginal parameters on the variation of melt pool and hatch overlap is discussed by comparing the top surface of specimens.

\subsection{Ti-6Al-4V powder properties}

In this study, Raymor Ti-6Al-4V powder was used in the SLM process. Currently, no literature reports on the optimized processing parameters for this specific fine powder, compared to EOS-provided powder. Fine powder is not suitable for the EBM process because small particles will be easily influenced by electrostatic charge, which results in 
serious powder spreading above the building platform (Kahnert et al, 2007). Therefore, Arcam Titanium Ti-6Al-4V powder was used for the EBM process.

\subsubsection{Raymor Ti-6Al-4V powder for SLM}

Raymor Ti-6Al-4V (Grade 23) powder has an apparent density of $2.55 \mathrm{~g} / \mathrm{cm}^{3}$. Most particles have diameter no more than $45 \mu \mathrm{m}(0-25 \mu \mathrm{m}, 34.1 \% ; 25-45, \mu \mathrm{m} 63.1 \% ;+45 \mu \mathrm{m}$, 2.8\%). Figure 2.3 shows the powder morphology under a Scanning Electron Microscope (SEM). The powder was measured using a Microtrac S3000 laser-based particle size analyzer. The spherical particles have a size distribution between $17.36 \mu \mathrm{m}\left(\mathrm{D}_{10}\right)$ and 44.31 $\mu \mathrm{m}\left(\mathrm{D}_{90}\right)$ with Mean Volume Diameter around $30 \mu \mathrm{m}$. Its particle size is nearly normally distributed around two peaks, with diameter $5.46 \mu \mathrm{m}$ and $28.67 \mu \mathrm{m}$. Raymor Ti-6Al-4V powder contains a lot of fine particles, compared to EOS Titanium Ti-6Al-4V powder (See Appendix B).

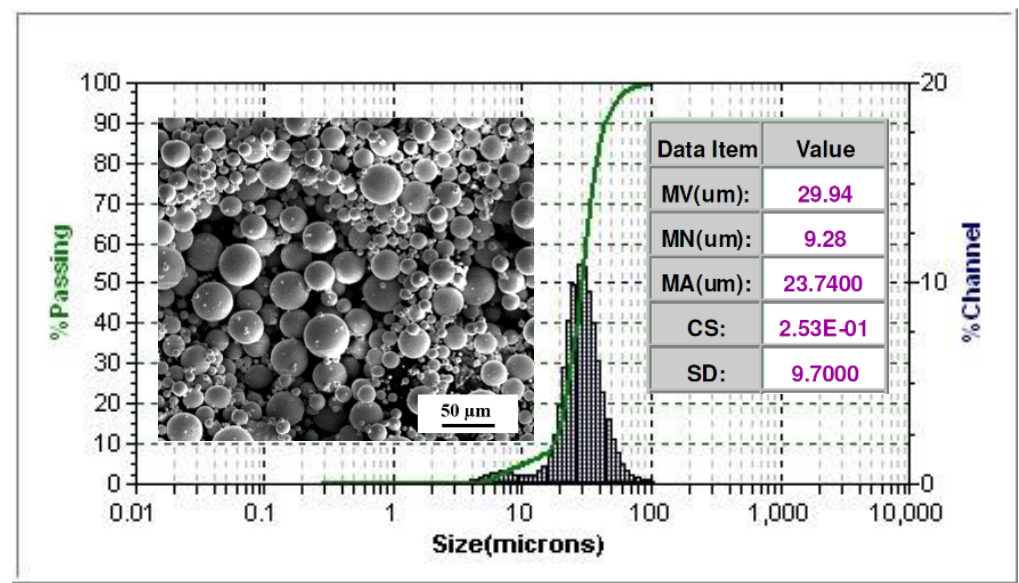

Figure 2.3. Raymor Ti-6Al-4V powder morphology and particle size distribution.

\subsubsection{Arcam Ti-6Al-4V powder for EBM}

Arcam Titanium Ti-6Al-4V ELI powder has a spherical morphology, with apparent density no more than $2.7 \mathrm{~g} / \mathrm{cm}^{3}$. The particle size analysis showed that Arcam powder has 
a size distribution between $46.94 \mu \mathrm{m}\left(\mathrm{D}_{10}\right)$ and $99.17 \mu \mathrm{m}\left(\mathrm{D}_{90}\right)$ with Mean Volume Diameter around $72.69 \mu \mathrm{m}$. The particle size is nearly normally distributed. But it has a small percentage of particles with diameter larger than $100 \mu \mathrm{m}$, as shown in Figure 2.4.

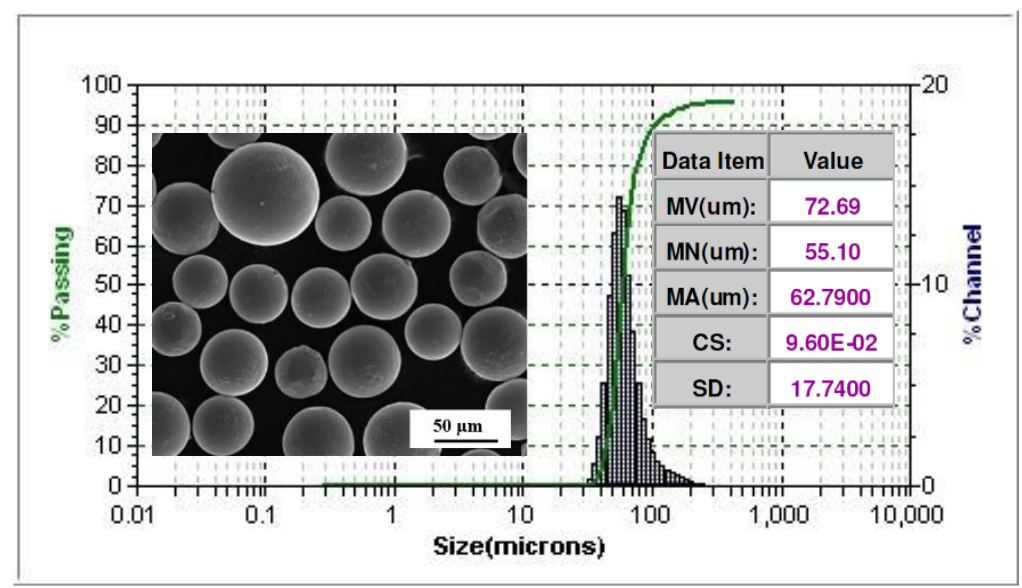

Figure 2.4. Arcam Ti-6Al-4V powder morphology and particle size distribution.

\subsection{Design of Experiments}

Energy density is usually used to describe the average applied energy per volume of material during a powder bed fusion process. Significant processing parameters are included in an energy density equation. For example, energy density can be expressed by Equation (2.1) for the SLM process (Thijs et al, 2010).

$$
E=\frac{P}{v \cdot h \cdot t}
$$

where $P$ is laser power, $v$ is scan speed, $h$ is hatch spacing and $t$ is layer thickness. Generally, these parameters have a complicated mutual effect during the laser melting process when all of parameters are varied in an experiment. The melt pool variation and defect generation will be easily characterized if some parameters are kept constant. According to the Equation (2.1), it is noted that laser power and scan speed have an inverse impact on the energy density. So this experiment adopted a factorial design based on these two 
parameters in order to correlate the energy density and defect regularity. An EOS M270 Direct Metal Laser Sintering (DMLS) system was utilized to fabricate specimens. The experimental factors and levels are given in Table 2.1.

Table 2.1. Factors and Levels of Factorial Experiment (SLM: Ti-6Al-4V, Hatch Spacing $100 \mu \mathrm{m}$, Layer Thickness $30 \mu \mathrm{m}$ )

\begin{tabular}{lc}
\hline \multicolumn{1}{c}{ Factor } & Level \\
\hline Laser Power $(\mathrm{W})$ & $40,80,120,160$ \\
Scan Speed $(\mathrm{mm} / \mathrm{s})$ & $120,240,360, \ldots, 1560$ \\
\hline
\end{tabular}

For the EBM process, "energy density" can also be expressed similarly as Equation (2.1), by substituting electron beam power for laser power (Kahnert et al, 2007). An Arcam S400 EBM system was used for this study. However, the scan speed cannot be directly varied in the Arcam system. It is dynamically controlled through a "speed function" during the melting process in order to achieve the correct melt pool size. The speed function is related to the "max current" and "speed function index". Additionally, "line offset" and "focus offset" are also influential processing parameters to the EBM process. Therefore, this study utilized max current, line offset, focus offset, and speed function index as the primary experimental factors. As shown in Table 2.2, three levels for each factor were selected for studying their significance on the defects through a Taguchi experiment (L9). The One-Factor-at-A-Time (OFAT) method was also employed to further investigate the significant factors.

Table 2.2. Factors and Levels of Taguchi Experiment (EBM: Ti-6Al-4V)

\begin{tabular}{lc}
\hline \multicolumn{1}{c}{ Factor } & Level \\
\hline Max Current $(\mathrm{mA})$ & $10,20,30$ \\
Line Offset $(\mathrm{mm})$ & $0.15,0.20,0.25$ \\
Focus Offset $(\mathrm{mA})$ & $5,10,15$ \\
Speed Function Index & $60,120,180$ \\
\hline
\end{tabular}


After SLM and EBM specimens were built, each specimen's density was measured using the Archimedes method (Spierings et al, 2011) according to ASTM B962-08. The measured density was compared with the nominal density of the Ti-6Al-4V material to estimate porosity according to Equation (2.2),

$$
R_{D}=1-\frac{\rho_{e}}{\rho_{n}}
$$

where $R_{D}$ is porosity, $\rho_{e}$ is measured density, and $\rho_{n}$ is nominal density. It can be seen that, the lower the measured density, the larger the porosity.

\subsection{Results and Discussion}

In this section, the cause of defect generation during SLM and EBM processing is analyzed and compared. Processing parameters are further classified based on their effect on the porosity generation. Microscopy of specimen's top surface illustrates the change of melt pool and overlap between hatch lines.

\subsubsection{Effect of Processing Parameters on SLM Ti-6Al-4V}

\subsubsection{Porosity Distribution and ANOVA}

Details of porosity variations with uncertainty value are respectively illustrated at different laser power in Figure 2.5. From Figure 2.5 (a), it can be seen that there is no apparent porosity when scan speed is between $600 \mathrm{~mm} / \mathrm{s}$ and $1600 \mathrm{~mm} / \mathrm{s}$ at a laser power of $160 \mathrm{~W}$. When the scan speed is less than $600 \mathrm{~mm} / \mathrm{s}$, which is a higher energy density, the increased porosity implies internal defects in the specimens. Moreover, porosity is not available when the scan speed is decreased below $360 \mathrm{~mm} / \mathrm{s}$, because specimens were not successfully built. The deposited layer was seriously deformed resulting in crash with the 
recoating blade. When laser power is at $120 \mathrm{~W}$ in Figure 2.5 (b), slow scan speed $(<360$ $\mathrm{mm} / \mathrm{s}$ ) also causes build failure. There could be no porosity or internal defects, when the scan speed ranges from 720 to $1200 \mathrm{~mm} / \mathrm{s}$. After that, the reduced energy density generates insufficient melting temperature in the Ti-6Al-4V powder, and the porosity increased with the scan speed.
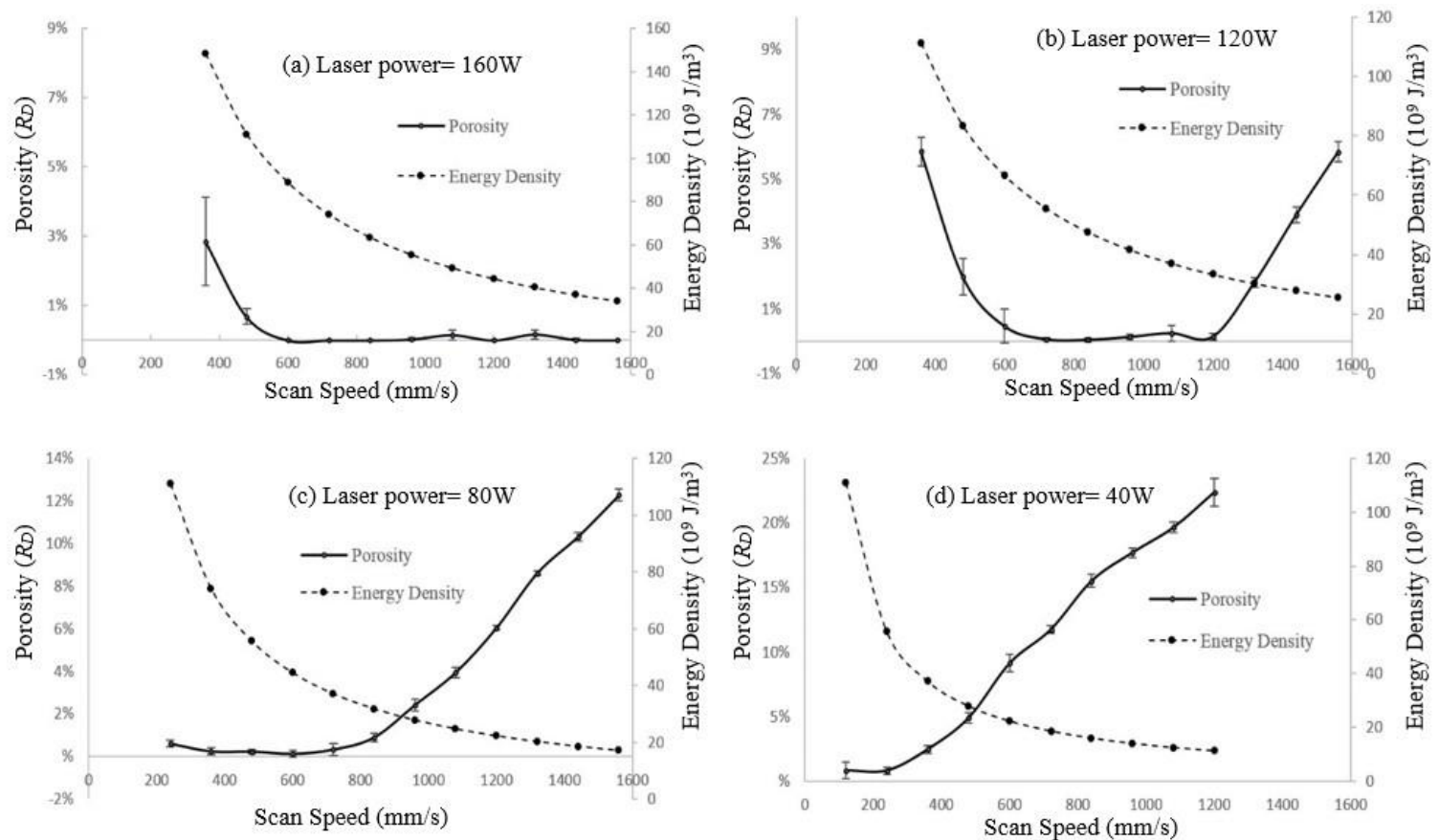

Figure 2.5. Porosity of SLM-produced Ti-6Al-4V specimen.

If the laser power is reduced to $80 \mathrm{~W}$, a porosity free specimen is only available when the scang speed is around $600 \mathrm{~mm} / \mathrm{s}$, as shown in Figure 2.5 (c). Lowering the scang speed leads to a small porosity. But the porosity is dramatically increased when increasing the scan speed. When the laser power is lowered to $40 \mathrm{~W}$, all specimens are assumed to contain defects even if at a slow scan speed. At $1200 \mathrm{~mm} / \mathrm{s}$, the specimen's porosity is even higher than 20\%, as shown in Figure 2.5 (d). Figure 2.6 shows the porosity distribution of SLM produced Ti-6Al-4V specimens made by factorial experiment. 


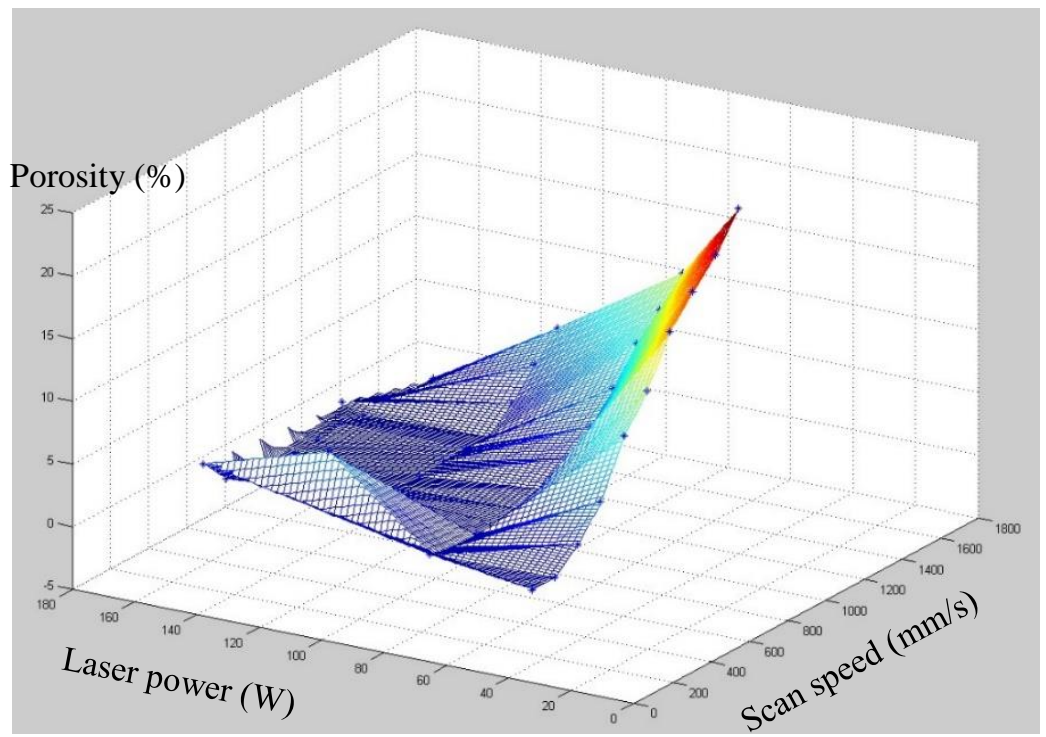

Figure 2.6. Porosity distribution of SLM factorial experiment.

Not all the specimens were successfully fabricated. Thus, the experimental results were trimmed to a full factorial experiment by eliminating some parameter combinations for ANOVA, which is summarized in Table 2.3. It can be seen that the $F$ values of laser power, scan speed, and their interaction are all larger than the threshold $\left(F_{0.05,3,96}, F_{0.05,12}\right.$, 96 , and $F_{0.05,21,160}$, which indicate significance with $95 \%$ confidence level.

Table 2.3 ANOVA of Factorial Experiment

\begin{tabular}{cccccc}
\hline Source & $\begin{array}{c}\text { Degree of } \\
\text { freedom }\end{array}$ & $\begin{array}{c}\text { Sums of } \\
\text { Squares (SS) }\end{array}$ & $\begin{array}{c}\text { Adj. Sums of } \\
\text { Squares (SS) }\end{array}$ & $\begin{array}{c}\text { Adj. Mean } \\
\text { Square (MS) }\end{array}$ & F value \\
\hline Laser Power & 3 & 0.34 & 0.34 & 0.11 & 6640.03 \\
Scan Speed & 7 & 0.04 & 0.04 & 0.01 & 322.18 \\
Power*Speed & 21 & 0.13 & 0.13 & 0.01 & 367.82 \\
Error & 96 & 0.00 & 0.00 & 0.00 & \\
Total & 127 & 0.51 & & & \\
\hline
\end{tabular}

$\mathrm{F}_{0.05,3,96}=2.70 ; \mathrm{F}_{0.05,7,96}=2.10 ; \mathrm{F}_{0.05,21,96}=1.67$

The laser power is the most significant factor during SLM. Scan speed also plays an important role in defects generation. The significance of interaction between laser power and scan speed partially verifies the influence of energy density to the SLM process. This 
conforms to the results of references mentioned in the literature review section with respect to SLM processing parameters.

\subsubsection{Process Window and Marginal Parameters}

According to the porosity distribution, a process window can be introduced to show the effect of laser powers and scan speeds on defects when laser melting Raymor Ti-6Al4V powder. SLM processing parameters are classified into "fully dense parameters" (Zone I), “over melting parameters" (Zone II), “incomplete melting parameters” (Zone III), and “over heating parameters" (Zone $\mathrm{OH}$ ), as shown in Figure 2.7. The energy density of each point in the process window could be simply represented by slope of a line between the origin and a point on the graph, if the hatch spacing and layer thickness are constant. For example, all points along the dash line in Figure 2.7 have the same energy density. The higher the slope value, the larger the energy density.

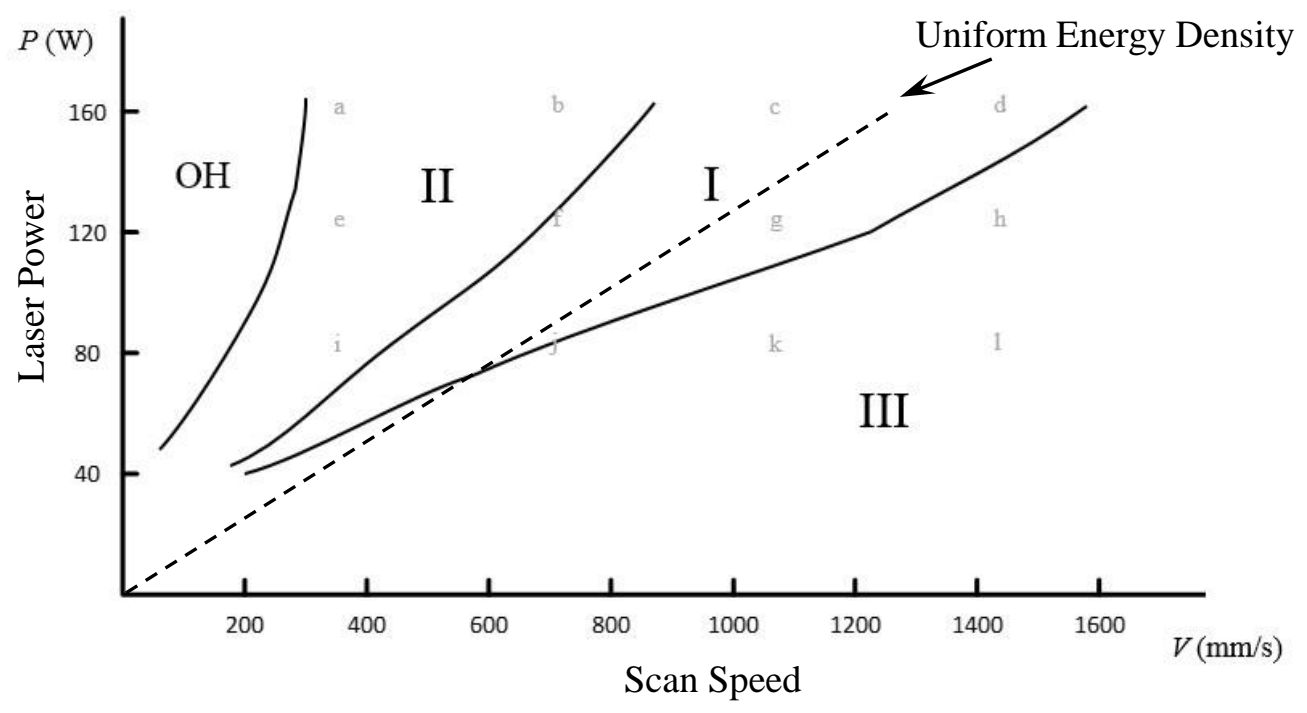

Figure 2.7. Process window for SLM Raymor Ti-6Al-4V powder. 
The Zone I parameters are capable of building fully dense specimens with Raymor Ti-6Al-4V powder. It is notable that energy density should be sufficient to avoid incomplete melting. In addition, even if the energy density meets the minimum requirement, porosity could also be included in the specimen. For example, the lower portion of the dashed line in Figure 2.7 demonstrates that these laser power and scan speed cannot be used to fabricate porosity free specimen. Specimens may not be built using Zone $\mathrm{OH}$ parameters. Because laser irradiation produces a large quantity of heat which is unable to be conducted away from the specimen immediately. The accumulated strain energy results in serious deformation causing collision with the recoating unit.

Zone II and III parameters can be used to fabricate Ti-6Al-4V specimens with porosity. These parameters are known as "marginal parameters" in this study because they are distributed to the margins of Zone I. It is a straightforward situation that insufficient energy density causes smaller melt pools and partially molten powder in Zone III. Thus, voids among powder particles are entrapped under the solidified hatch lines so that the overall density of the specimen is lowered. However, defects also exist in the specimens fabricated by the Zone II parameters. This implies that extra energy not only causes microscopic structural rearrangements of SLM Ti-6Al-4V, but also a complex transport phenomenon of macroscopic masses over macroscopic distances, with ablation and compression of material and with large internal energies compared to chemical activation energies (Von Allmen et al, 1987).

In order to analyze the melting process under different processing parameters, typical solidified top surface were observed under SEM, as shown in Figure 2.8. Each picture corresponds to the processing parameter combination specified in Figure 2.7. 

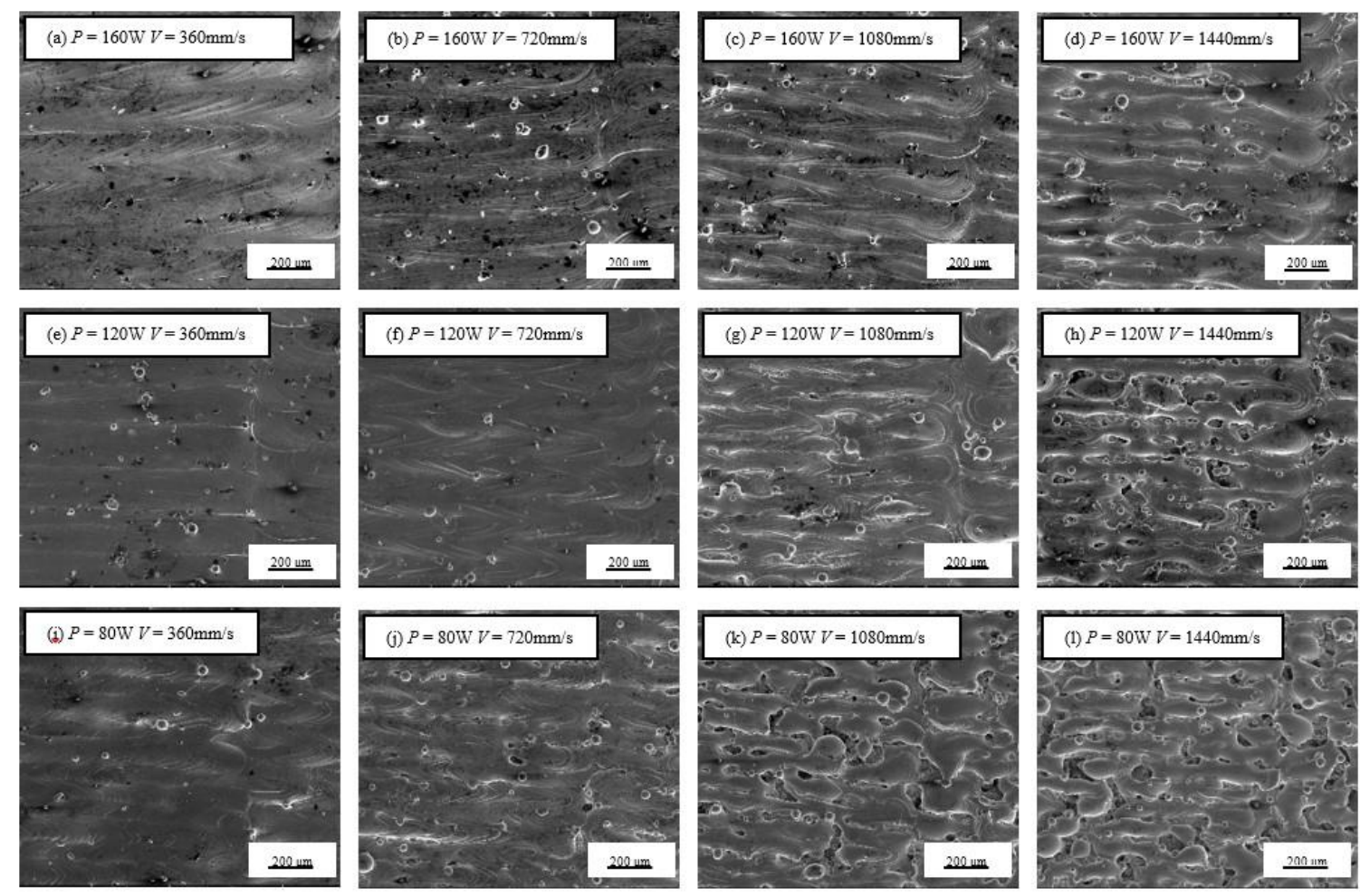

Figure 2.8. Scanning electron microscopy of top surface of laser melted Ti-6Al-4V.

In Zone I, the processing parameters are able to build fully dense specimens. Figure 2.8 (c), (d), (f) and (g) show the solidified hatch lines of these specimens. With increasing the scan speed, the melt pool size is gradually reduced. Because hatch spacing is constant, the reduced melt pool size results in decreased overlap, which deteriorates the top surface's roughness, as shown in Figure 2.8 (d) and (g). Although voids occasionally appear due to discontinuous hatch lines, they are eliminated by the following recoating and melting process. Thus, no apparent porosity was detectable in the specimens through Archimedes method and destructive characterization techniques. Fig 2.8 (a), (b), (e) and (i) show the top surface melted by the Zone II marginal parameters. The melt pool was extremely extended with high energy density, especially in the ends of each hatch line due to the ramping phenomenon of scan speed. On the other hand, energy deficiency causes the 
melting discontinuity when using the Zone III marginal parameters. As shown in Figure $2.8(\mathrm{~h}),(\mathrm{j}),(\mathrm{k})$ and (l), many breaking spots are visible along the hatch lines.

\subsubsection{Defects Caused by Zone II Marginal Parameters}

A large melt pool results in the increased overlap between hatch lines. However, it can be seen that the highly overlapped hatch lines do not necessarily form voids or defects on the remelted top surface. A certain amount of spherical particles and pits are observable on a solidified top surface, as shown in Figure 2.9. It is believed that the porosity of specimens built by the Zone II marginal parameters is directly correlated with these particles and pits. When melting the Ti-6Al-4V powder at higher energy density, laser irradiation not only causes larger melt pool, but also extra heat which evaporates the molten materials. The melt pool is subject to recoil force from the evolving vapor, which ejects molten materials (Kruth et al, 2004). This mechanism is similar to the ablating process of laser drilling. But the materials are even more easily extracted from a powder bed.

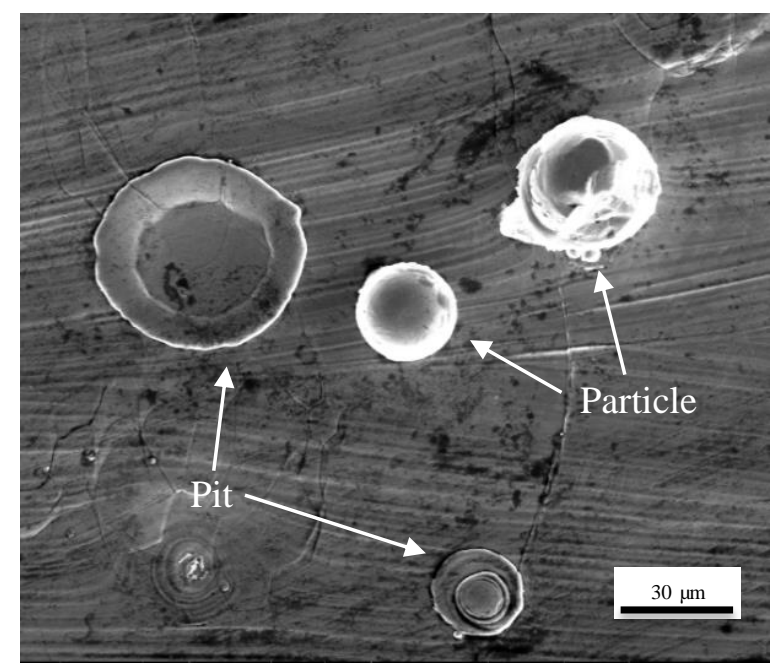

Figure 2.9. SEM of pits and particles on top surface of SLM specimen. 
The molten metal rapidly solidifies above the building platform and forms spherical particles, some of which fall down and are then welded together with the top surface of the specimens. Small welded particles will be melted together with recoated powder particles. However, large particles, which attach to the top surface, will be easily touched and removed by the recoating blade, as shown in Figure 2.10 .

Pits will be formed with a hollow spherical crown shape after removal. Large pits could be filled with recoated powder. But small pits could be easily entrapped underneath new layers as defects. Due to their occasional formation, these defects are distributed stochastically in the specimen, instead of directly being correlated with processing parameters. This explains why the uncertainty value of specimen porosity built by the Zone II marginal parameters is larger than that by the Zone III marginal parameters.

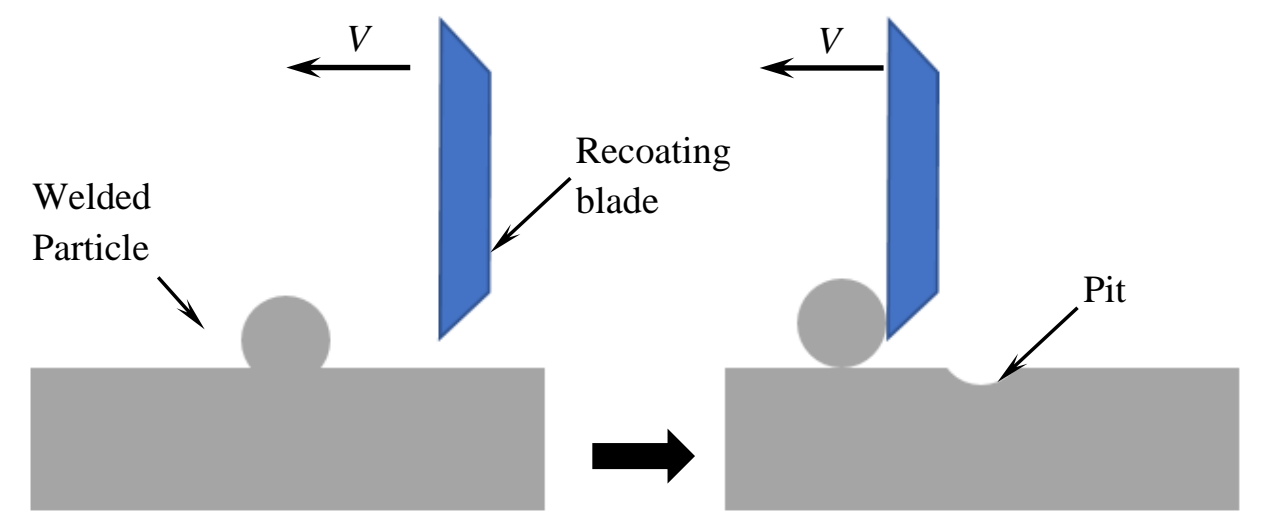

Figure 2.10. Schematic of defect caused by recoating during SLM process.

\subsubsection{Defects Caused by Zone III Marginal Parameters}

The discontinuity of hatch lines results from the velocity inequality between laser scan speed and melt pool interface motion. When laser power is applied to the powder bed, the powder particles form a melt pool in a transient process. Temperature of the interface between the molten material and un-melted powder is around the melting point $\left(1660{ }^{\circ} \mathrm{C}\right.$ 
for Ti-6Al-4V). If there is no enough energy furnished to the interface to preserve melting and steady melt pool, the velocity of the interface will be occasionally slower than that of the laser spot motion (i.e. $V_{\text {melt pool }}<V_{\text {laser }}$ ), as illustrated in Figure 2.11 (a). Thus, hatch lines can hardly be formed continuously. Moreover, due to the reduced melt pool size, the overlap between two hatch lines is very limited, which exacerbates the wetting condition for the following layer. If the molten material fails to wet on the previous layer and adjacent hatch line (Figure 2.11 (b)), it will easily accumulate to form a rounded surface due to surface tension. Therefore, the voids or pores are seriously generated.
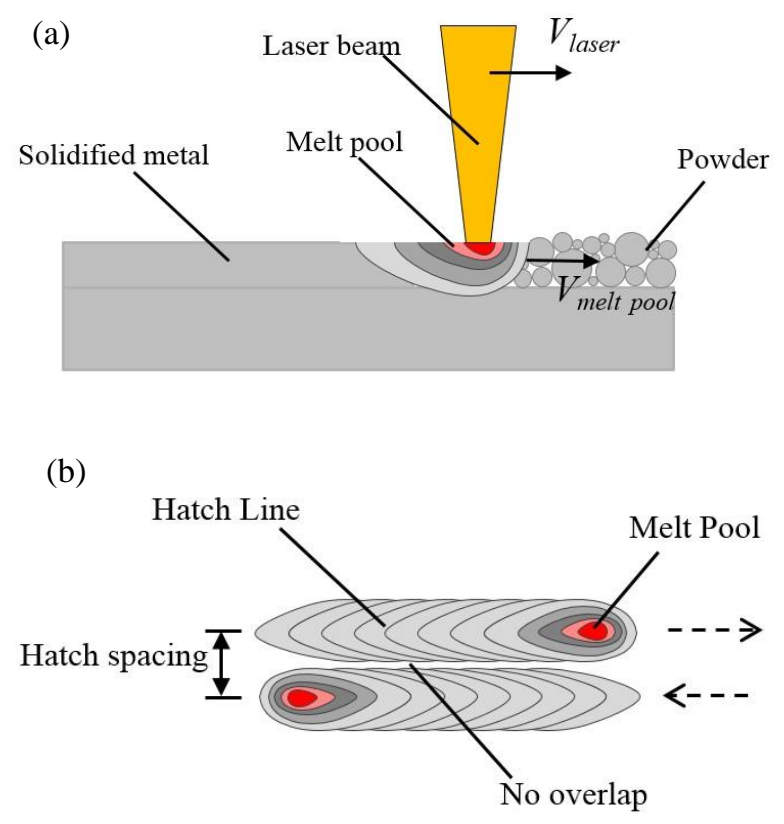

Figure 2.11. Schematic of defect daused by Zone III marginal parameters of SLM process. (a) Laser melting metallic powder; (b) top view of two adjacent hatch lines.

A typical solidified surface is shown in Figure 2.12, which was fabricated by the Zone III marginal parameters. The scanning direction is indicated in the figure. Some powder particles were sintered on the top surface which are visible through the voids. The voids are randomly distributed with irregular morphology along and between hatch lines. 


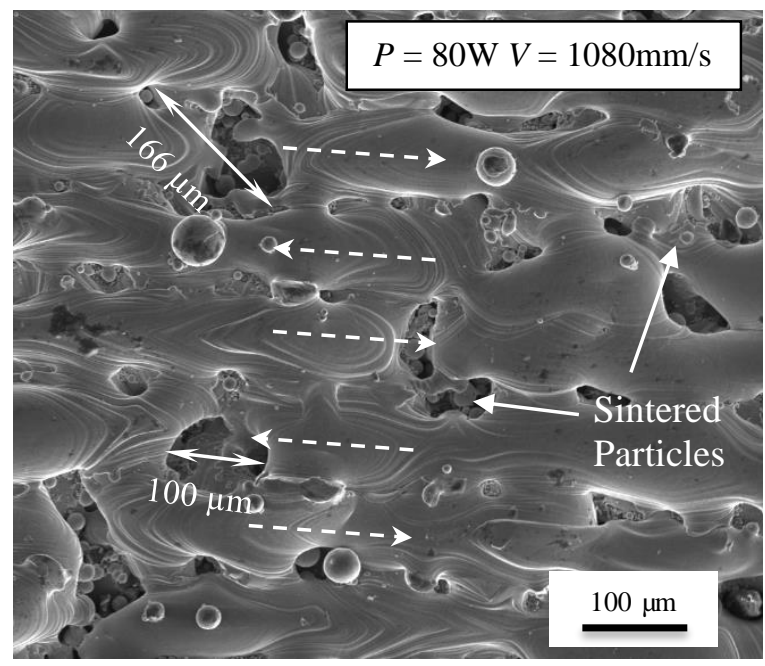

Figure 2.12. Top surface fabricated by Zone III marginal parameters of SLM process.

\subsubsection{Effect of Processing Parameters on EBM Ti-6Al-4V}

\subsubsection{Mean Porosity Distribution and ANOVA}

EBM specimens' mean porosity versus individual experimental factor is shown in

Fig 2.13. The porosity for each level of a particular factor corresponds to an average of three experimental runs at that level, typically with four replicates, representing an average of 12 density measurements.
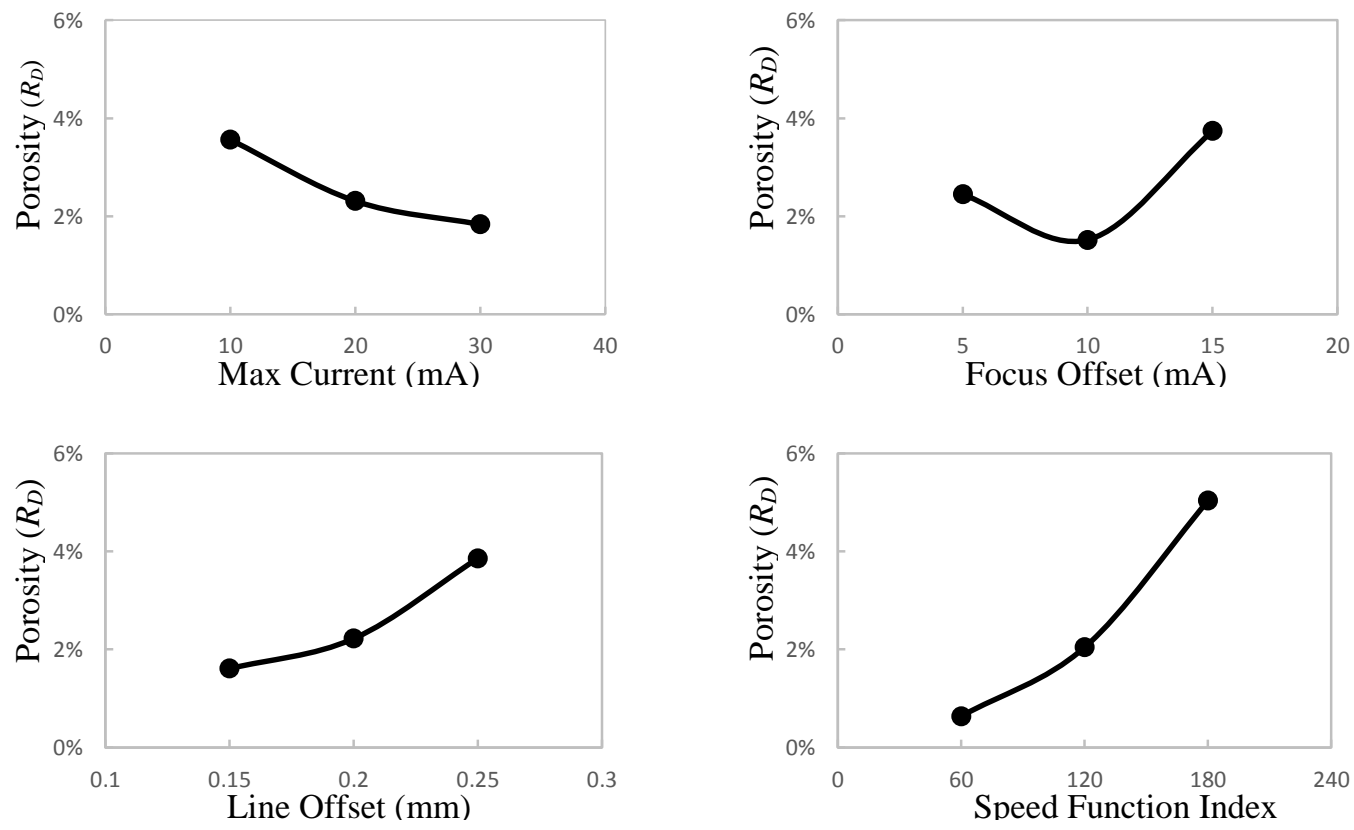

Figure 2.13. Mean porosity at each level of taguchi experimental factors. 
ANOVA was also performed to statistically evaluate the effect of each parameter on porosity of EBM Ti-6Al-4V specimens. The results of ANOVA are summarized in Table 2.4. It can be seen that all $F$ values are larger than $F_{0.05,2,27}$. Thus, all experimental parameters could be considered as significant factors influencing the porosity (95\% confidence level). The speed function number has the most significant influence on specimens' porosity, followed by line offset, focus offset, and max current.

Table 2.4 ANOVA of Taguchi Experiment

\begin{tabular}{ccccc}
\hline Source & $\begin{array}{c}\text { Degree of } \\
\text { freedom }\end{array}$ & $\begin{array}{c}\text { Sums of Squares } \\
\text { (SS) }\end{array}$ & $\begin{array}{c}\text { Mean Square } \\
(\text { MS })\end{array}$ & $\begin{array}{c}\text { F } \\
\text { value }\end{array}$ \\
\hline Max Current & 2 & 0.04 & 0.02 & 58.94 \\
Focus Offset & 2 & 0.06 & 0.03 & 93.14 \\
Line Offset & 2 & 0.06 & 0.03 & 102.02 \\
Speed Func. No. & 2 & 0.23 & 0.12 & 376.73 \\
Error & 27 & 0.01 & 0.00 & \\
Total & 35 & 0.40 & & \\
\hline
\end{tabular}

$\mathrm{F}_{0.05,2,27=3.35}$

However, it is difficult to correlate the speed function index with actual energy input based on the existing references. Thus, line offset, focus offset and max current were studied by using OFAT method with a constant speed function index (default 98 for EBM Ti-6Al-4V powder) at layer thickness $50 \mu \mathrm{m}$. The OFAT experiments also illustrate the significance of line offset and focus offset to porosity change. Based on the porosity distribution, the increased line offset and focus offset could also be considered marginal parameters. Otherwise, there is no apparent porosity fabricated by varying max current. It is assumed that the Arcam system is able to regulate the scan speed automatically according to the powder bed temperature, even at the reduced beam current. Thus, analysis and discussion about EBM processing parameters are mainly focused on line offset and focus offset. 


\subsubsection{Defects Caused by the Increased Line Offset}

Porosity distribution versus line offset is shown in Figure 2.14. It can be seen that the porosity is increased by increasing line offset, especially when the line offset is larger than $0.18 \mathrm{~mm}$.

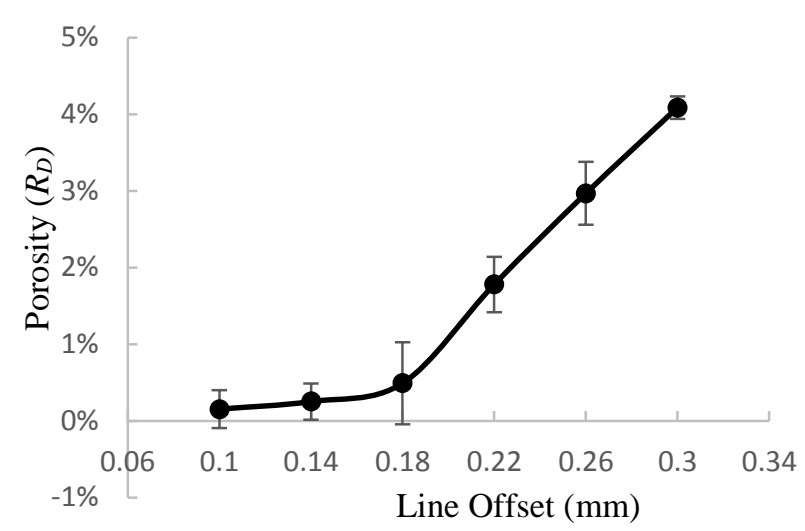

Figure 2.14. Porosity versus line offset.

Line offset refers to the distance between two hatch lines, similar to hatch spacing in SLM. According to the energy density equation, increased line offset will reduce the energy density value. The OFAT experimental result indicates that porosity is dramatically increased when the line offset value is larger than $0.18 \mathrm{~mm}$. In order to correlate line offset with defect generation, solidified hatch lines are shown in Figure 2.15.

When line offset is $0.1 \mathrm{~mm}$ (default value), the solidified hatch lines are arranged equally. No apparent pores or defects are formed between hatch lines. However, overlap between hatch lines will be reduced if the line offset is increased to 0.14 and $0.18 \mathrm{~mm}$. Although there are no visible pores or voids on the top surface (Figure 2.15 (b) and (c)), a small amount of porosity is detectable from the porosity curve shown in Figure 2.14. 

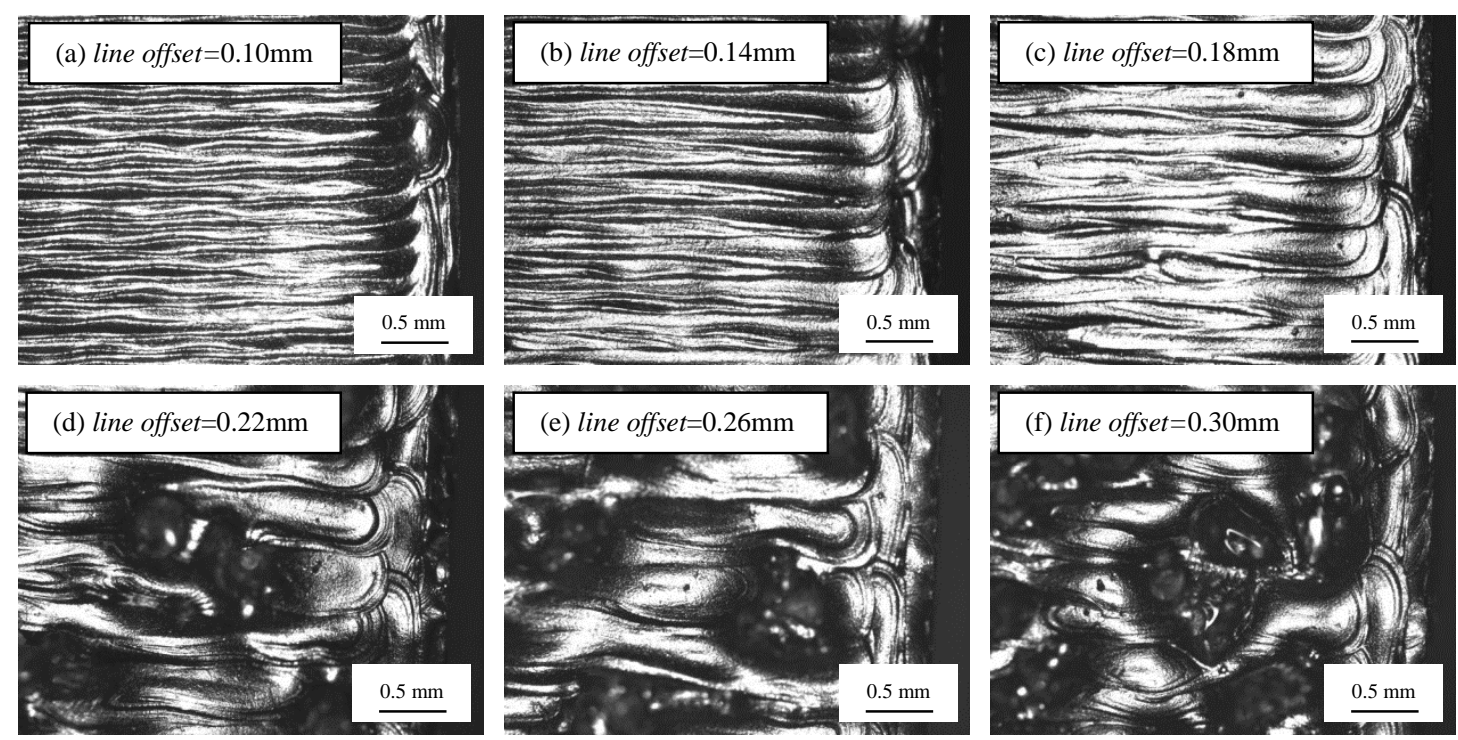

Figure 2.15. Solidified top surfaces of EBM specimens by varying line offset.

This could be attributed to the un-melted powder under the adjacent hatch lines, as shown in Figure 2.16. The powder gap was trapped inside the specimen as defects. This means not all powder is melted during the EBM process if the line offset value is increased.

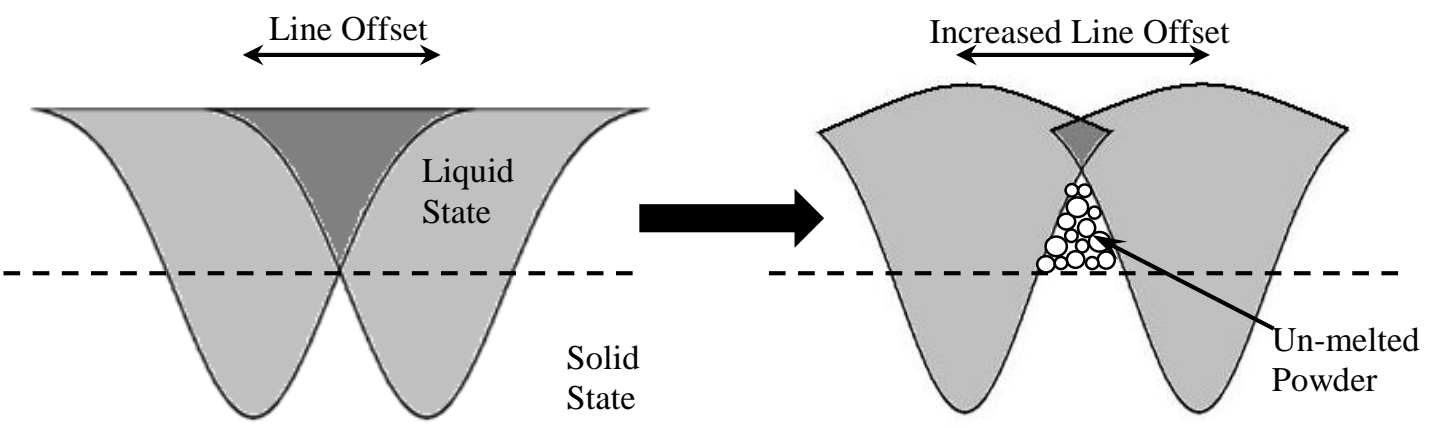

Figure 2.16. Schematic of melt pool with increased line offset during EBM process.

The porosity is not obvious until the line offset is increased to $0.22 \mathrm{~mm}$. Apparent defects are observable on the surface, as shown in Figure 2.15 (d). When line offset is increased to $0.26 \mathrm{~mm}$ and $0.30 \mathrm{~mm}$, the melt pool fails to contact the previous hatch line, which leads to bad wetting conditions. In order to reduce total surface free energy, the 
melted powder easily gathers together and flows above the un-melted powder, causing voids between hatch lines, as shown in Figure 2.15 (d)-(f). The un-melted powder is visible through the pores of the top surface. The dual effects of voids and powder gap seriously increase the porosity of specimens made with a large line offset value. According to the solidified surface, it can be inferred that melt pool width is larger than $0.18 \mathrm{~mm}$, but less than $0.22 \mathrm{~mm}$.

\subsubsection{Defects caused by the Increased Focus Offset}

Porosity distribution versus focus offset are shown in Figure 2.17. The focus offset value does not significantly change the porosity until $16 \mathrm{~mA}$.

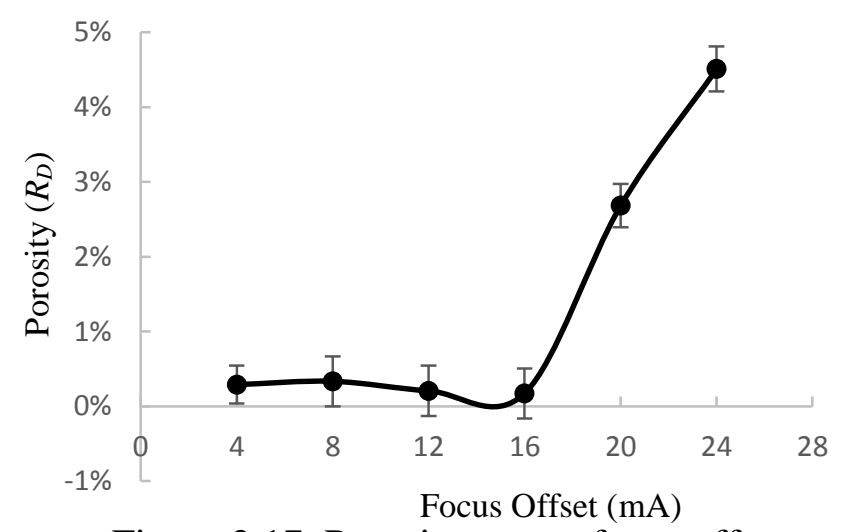

Figure 2.17. Porosity versus focus offset.

Focus offset is the additional current running through the respective electromagnetic coil and can be translated into an offset of the focal plane from its zero position (Schwerdtfeger et al, 2012). Increasing the focus offset value results in a larger beam diameter during the EBM process. This can lead to an increased beam spot, but less energy concentration. Thus, energy density is reduced. Solidified surface was also observed under microscope and shown in Figure 2.18. 
A small focus offset value results in concentrated electron beam spot and profound melting depth. Thus, the penetration depth of an electron beam into the powder layer is greater than that of the laser beam (Zäh et al, 2010). Previous solidified titanium is also melted to form melt pools which overlap each other to ensure hatching consistency. Each solidified hatch line forms a ridge shape and causes a rough surface. By increasing the focus offset value, the morphology of the melt pool is changed. As shown in Figure 2.19, the horizontal melt pool size is enlarged, while the melting depth is reduced.
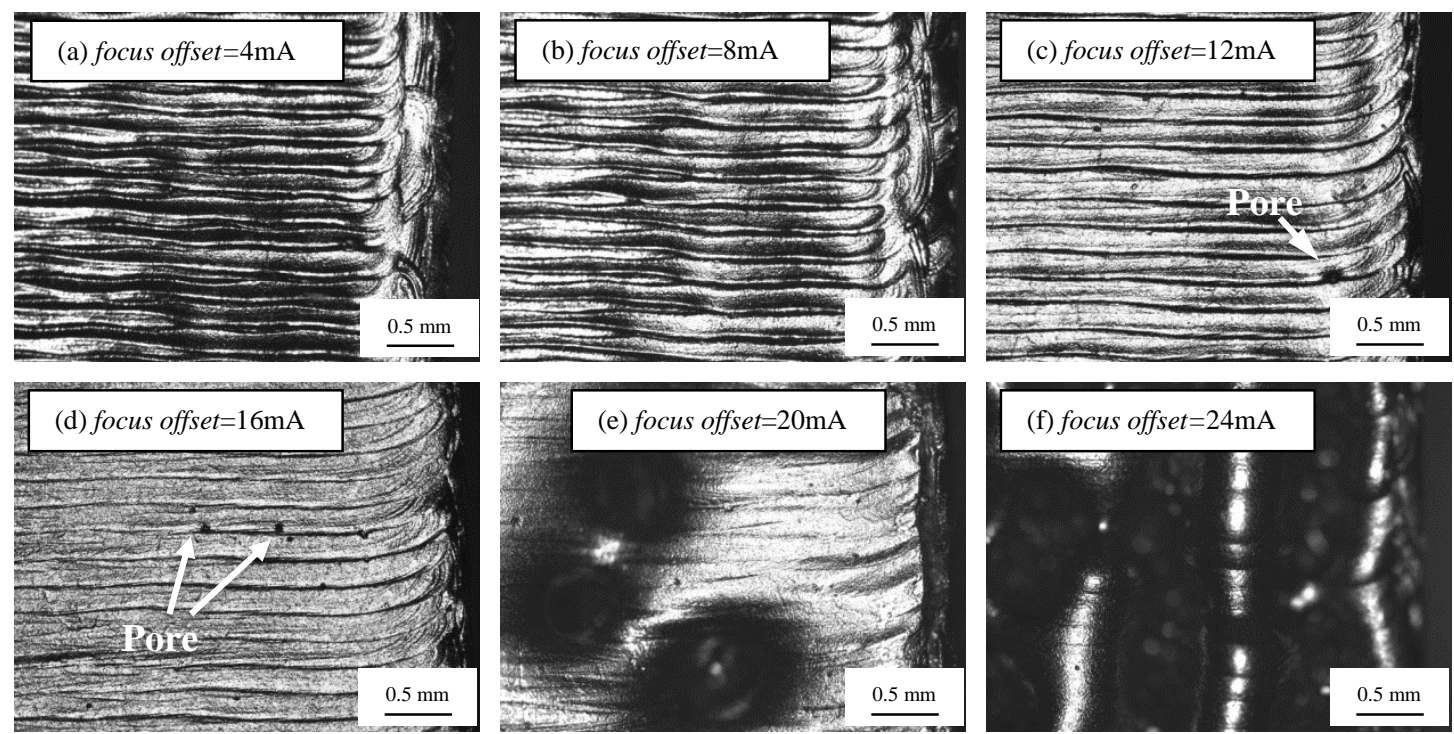

Figure 2.18. Solidified top surfaces of EBM specimens by varying line offset.

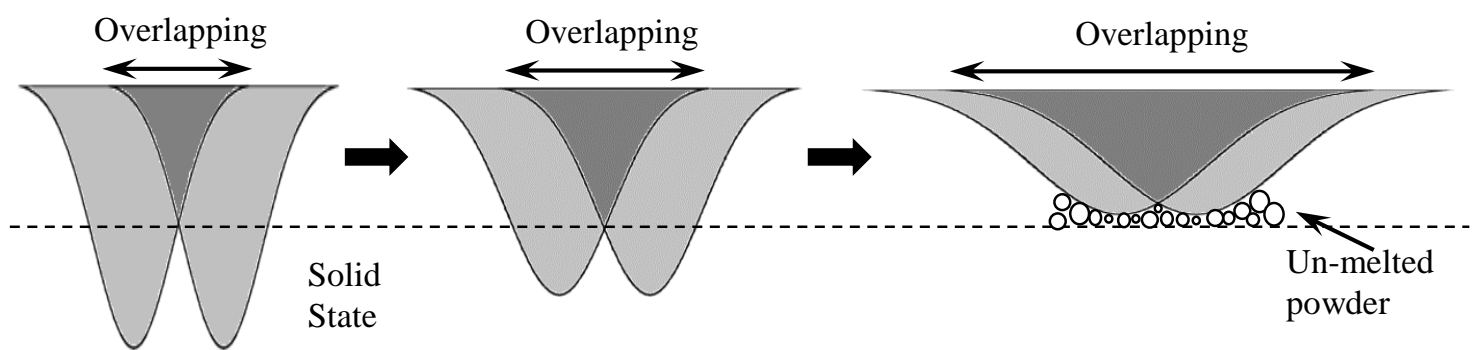

Figure 2.19. Schematic of melting pool with increased focus offset during EBM process. 
Increased overlap area improves the surface roughness significantly as shown in Figure 2.18 (c, d). But small pores may be generated at localized areas. These spherical pores result from gaseous argon entrapped in the powder particles caused by the atomization process (Biamino et al, 2011). The melted powder releases the trapped gas which causes the small defects. However, these defects are barely detectable using the Archimedes method. When focus offset value is higher than $16 \mathrm{~mA}$, the increased overlap area and reduced melting depth create an unstable melt pool due to underneath un-melted powder, as shown in Figure 2.19. The flowability of molten powder causes interstitial shortage which is reflected by the increased porosity.

\subsubsection{Comparison of Defect Formation between SLM and EBM}

Although SLM and EBM are similar in principle, defects are generated in different pattern when deviating processing parameters from optimized parameters. In the SLM process, high energy density causes over melting on the powder bed. The defects are resulted from the blade scraping particles, which are solidified from the ejected molten materials on the surface. Thus, the defects generation is mainly attributed to a physical removal process when using Zone II marginal parameters. By contrast, Arcam EBM system adopts a more complicated feedback control design, compared to EOS system. Higher energy density is hard to be achieved during the EBM process. Generally, no defects could be fabricated even though the electron beam power is increased. Beam current and scan speed are dynamically controlled to maintain a steady melt pool.

If the input energy fails to melt the metallic powder completely, the defects will be easily formed due to the voids between the remelted materials. In the SLM process, the 
Zone III defects are attributed to the breaking spots of hatch lines, or gaps between two hatch lines due to the reduced melt pool size. But the increased line offset or focus offset causes the melt pool in an unstable situation in the EBM process, because of the unmelted powder around melt pool. Serious flowability of the molten material results in large voids on the powder bed. Therefore, according to the microscopy, it is anticipated that EBM defects are larger than the SLM defects if energy density is lowered during the melting process.

\subsection{Conclusion}

Porosity distribution was investigated by varying processing parameters in this study. The change of processing parameters is directly related to the energy to the Ti-6Al$4 \mathrm{~V}$ powder bed. Although energy density has a significant effect on defect generation, it is not linearly correlated with porosity. Marginal parameters are responsible for the defect generation during SLM and EBM processes.

In the SLM process, splashed particles are generated by the Zone II marginal parameters and then welded to the top surface. The layer deficiency is caused by the recoater removing the large welded particles. Thus, Zone II marginal parameters indirectly generate defects, which are distributed stochastically in SLM specimens. Defects can be

directly formed by Zone III marginal parameters. The insufficient energy density results in voids along or between hatch lines.

For EBM process, the increased line offset and focus offset are similar to the Zone III marginal parameters of SLM. Defects are formed due to insufficient energy density. 
Interstices between un-melted particles account for a certain amount of porosity in the specimens, while the deteriorated hatch lines exacerbates the inclusion of defects. 


\section{CHAPTER 3}

\section{DEFECT MORPHOLOGY IN TI-6AL-4V PARTS FABRICATED}

\section{BY SLM AND EBM ${ }^{3}$}

\subsection{Introduction}

Selective Laser Melting (SLM) and Electron Beam Melting (EBM) are two common powder bed fusion processes within Additive Manufacturing (AM) for fabricating parts from metallic powders (Gibson et al, 2009). The SLM process selectively melts metallic powder using a focused laser beam. The melted powder attaches to the previous layer or support structure and solidifies in a short time. After that, a layer of new powder is recoated upon the current layer for the following layer melting (Thijs et al, 2010). At present, multiple materials can be utilized for SLM such as stainless steel, maraging steel, cobalt chromium and titanium alloys. The EBM process is similar. But, an electron beam, which is generated by a tungsten filament in the electron beam gun, is used instead of a laser beam. When the electrons penetrate the powder surface and further into the powder grains, their kinetic energy is converted into thermal energy (Zäh et al, 2010). Titanium Ti6Al-4V and cobalt chromium powder are well developed for EBM.

\footnotetext{
${ }^{3}$ This chapter is a paper published in the Solid Freeform Fabrication Symposium (2013). All permissions to use this paper as a part of this dissertation are contained in Appendix D.
} 
Numerous studies on SLM and EBM have been carried out for process and material development. Additively manufactured materials were also tested and compared to that made from conventional methods. Due to their distinguished layer adding process and rapid cooling rate, SLM and EBM materials exhibit special microstructures and outstanding mechanical properties. Ti-6Al-4V powders are widely used in SLM and EBM processes for their inherent properties of fracture resistance, fatigue behavior, corrosion resistance and biocompatibility (Soboyejo et al, 2006). However, defects can be easily formed in additively manufactured Ti-6Al-4V material depending upon the influence of multiple factors. Thus, an investigation of defect morphology is helpful for understanding their effects on material properties.

AM powder bed fusion processes can be regarded as a type of Powder Metallurgy (PM) process (Sam Froes, 2012), which are commonly used to produce porous parts. When an AM process is utilized to fabricate porous parts, it can be used to create structures which exhibit extreme flexibility in structural diversity and porosity characteristics if the morphology and distribution of defects in Ti-6Al-4V material is well-understood.

This chapter studies the dimensional distribution, morphology of defects, and porosity generated by varying processing parameters in SLM and EBM. The reasons for defect formation are discussed. Variations in defect morphology with different energy input are analyzed. The statistical results are thus a good reference for future comparisons with traditional PM-produced porous materials.

3.2 Material and method

3.2.1 Powder Property 
Raymor Ti-6Al-4V powder (Grade 23) with an apparent density of $2.6 \mathrm{~g} / \mathrm{cm}^{3}$ was used for SLM process. It has a particle size distribution between $17 \mu \mathrm{m}\left(\mathrm{D}_{10}\right)$ and $44 \mu \mathrm{m}$ (D90) with Mean Volume Diameter around $30 \mu \mathrm{m}$. Lots of fine particles are included in Raymor Ti-6Al-4V powder. Arcam Titanium Ti-6Al-4V ELI powder was used for EBM process. Its apparent density is no more than $2.7 \mathrm{~g} / \mathrm{cm}^{3}$. The particle size is normally distributed between $47 \mu \mathrm{m}\left(\mathrm{D}_{10}\right)$ and $99 \mu \mathrm{m}\left(\mathrm{D}_{90}\right)$ with a Mean Volume Diameter around $73 \mu \mathrm{m}$. Both Raymor and Arcam powder particles are spherical.

\subsubsection{Fabrication of Ti-6Al-4V Specimens}

Porosity $\left(R_{D}\right)$ of additively manufactured Ti-6Al-4V specimens can be estimated by measuring density using Archimedes method, and then compared with nominal density of Ti-6Al-4V material. Lower measured densities result in larger porosities. A process window, as shown in Figure 3.1, illustrates the effect of various laser power and scan speeds when using SLM to process Raymor Ti-6Al-4V powder.

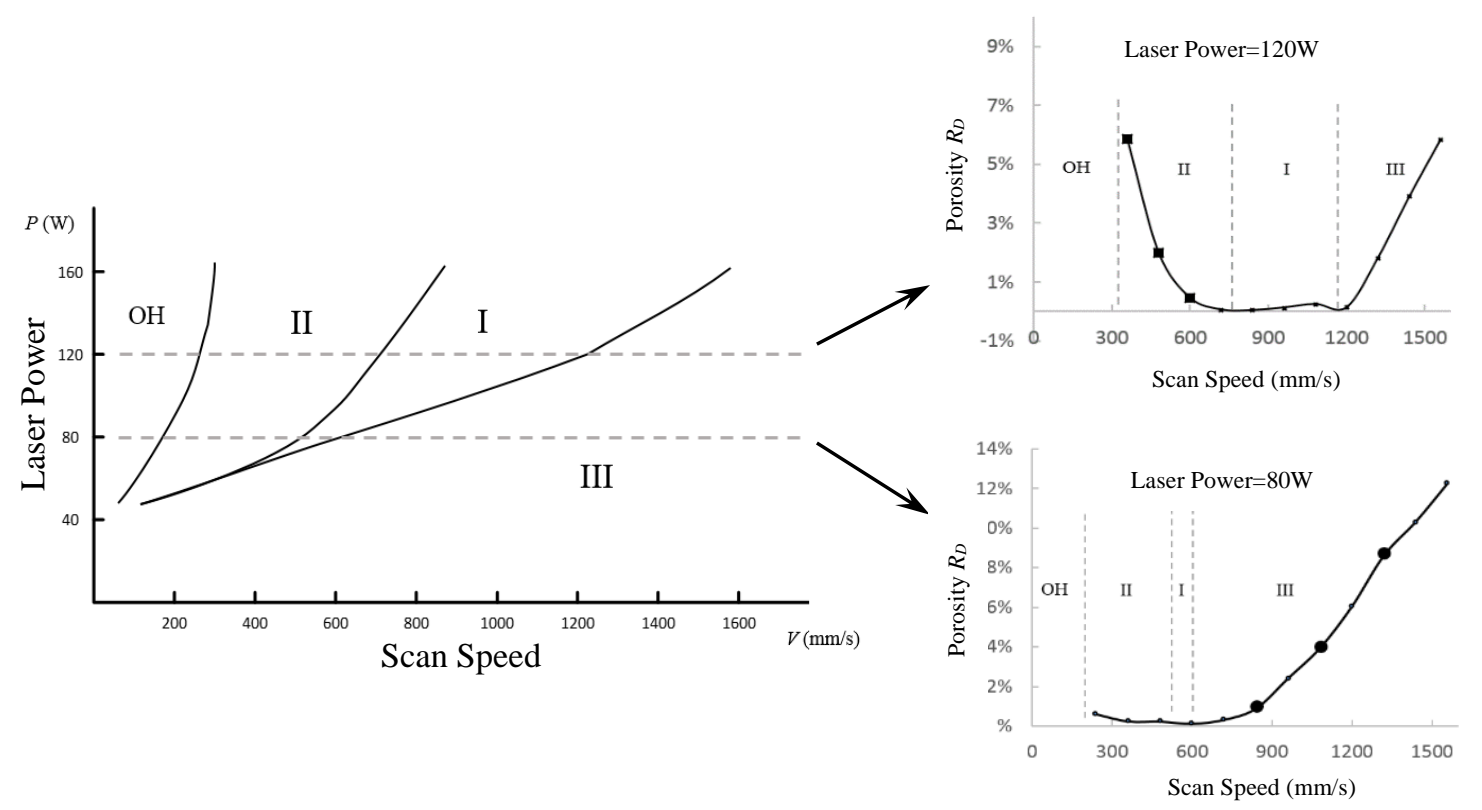

Figure 3.1. Process window of SLM and porosity distribution at $120 \mathrm{~W}$ and $80 \mathrm{~W}$. 
Processing parameters can be divided into four melting zones. Zone I parameters result in nominally fully dense specimens. Specimens built at a high energy densities with Zone II parameters result in over melting. Defects are often generated in these specimens. Ti-6Al-4V powder is not completely melted using lower energy Zone III parameters, which results in pores and voids. Specimens cannot be successfully built using Zone $\mathrm{OH}$ parameters due to serious thermal deformations.

In previous research, it was found that specimens can be built with various porosity distribution when using processing parameters, such as laser power and scan speed, from different melting zones. Thus, investigations about defect morphology are primarily based on specimens when the laser power is equal to $80 \mathrm{~W}$ and 120W, as shown in Figure 3.1. Zone III processing parameters at elevated scan speeds are of particular interest in this study because they are able to fabricate specimens with controlled porosity. An EOS M270 Direct Metal Laser Sintering (DMLS) system was utilized for building SLM specimens in this study.

An Arcam S400 EBM system was utilized in this study. Changes to beam current and scan speed independently do not cause porosity in specimens, because the Arcam system dynamically coordinates these two parameters to achieve a steady melt pool size. However, "line offset" and "focus offset" can be varied to make porous specimens. Line offset refers to the distance between two hatch lines. And focus offset is the additional current running through the respective electromagnetic coil and can be translated into an offset of the focal plane from its zero position and thus a change in beam area (Schwerdtfeger et al, 2012). Increased line offset results in lower energy densities, which forms voids in Ti-6Al-4V specimens. Increased focus offset increases beam diameter, thus 
lowering energy density and generating porosity. Figure 3.2 shows porosity distribution by varying line offset. It can be seen that the porosity value increases when the line offset value is increased, especially when the line offset is beyond $0.18 \mathrm{~mm}$. As shown in Figure 3.3 , apparent porosity (>0.5\%) appears when focus offset is larger than $16 \mathrm{~mA}$. Particularly at $20 \mathrm{~mA}$ and $24 \mathrm{~mA}$, the increased focus offset causes a sharp increase in porosity in Ti$6 \mathrm{Al}-4 \mathrm{~V}$ specimens. In order to investigate the defect characteristics, EBM specimens were built by increasing line offset and focus offset. In SLM and EBM processes, a number of processing parameters can be utilized to create specimens with a certain amounts of porosity, such as Zone II and III parameters of SLM or increased line offset and focus offset of EBM. These parameters are called "marginal parameters" in this study.

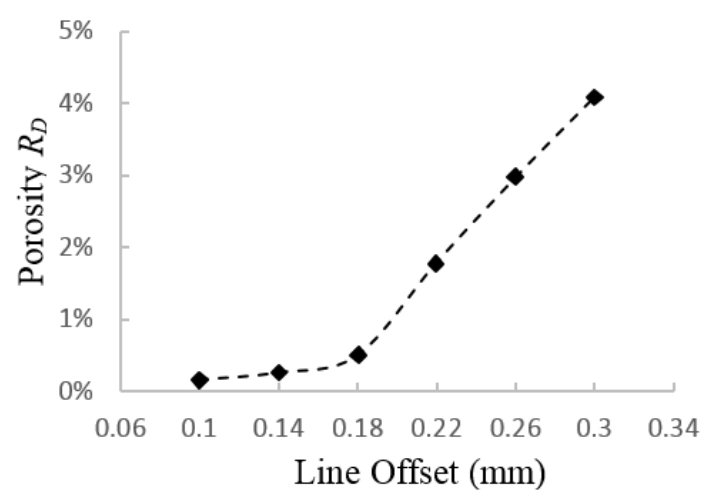

Figure 3.2. EBM porosity versus line offset.

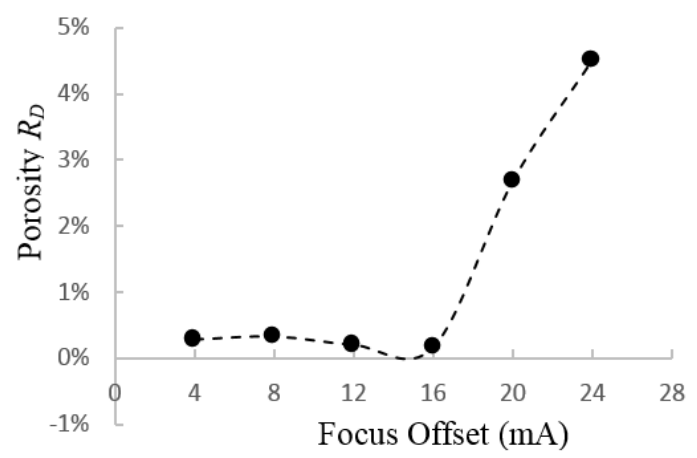

Figure 3.3. EBM porosity versus focus offset. 


\subsubsection{Image Processing Method}

For each marginal parameter combination, two cubical Ti-6Al-4V specimens were sectioned and polished for microscopy. One specimen was cut along the horizontal plane; the other along vertical plane, as shown in Figure 3.4. Cross sections were polished and then observed under optical microscope. Micrographs were taken for image processing process using Matlab R2012a.
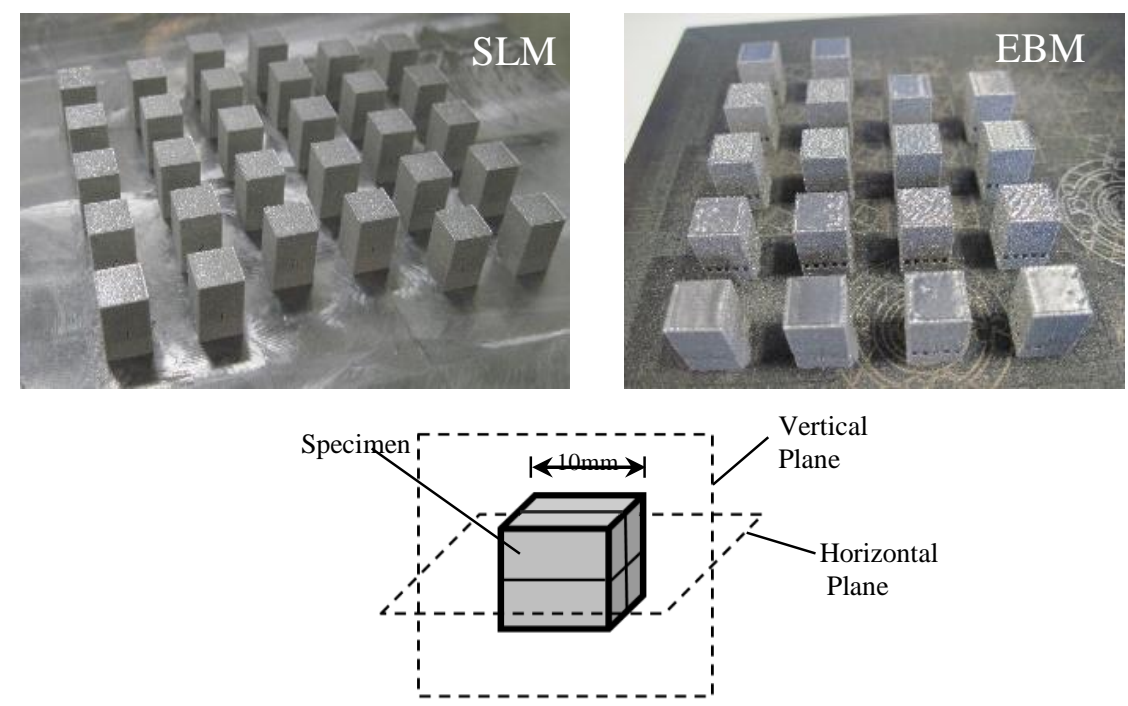

Figure 3.4. Ti-6Al-4V specimen and dimension

Micrographs were converted to binary images in order to highlight the features of the defective region. When a pixel's luminance of the original image is less than a userselected threshold value, the pixel will be replaced with a value of 1 and other pixels will be replaced with a value of 0 . In this paper, the threshold value is selected as 0.2 . By doing this, the solid region is shown as a black color, while defective areas are white. Images which included contamination or polishing scratches were "cleaned" using a de-noising algorithm. For each defect, an area value could be obtained by counting pixels and then multiplying by individual pixel area. All defect areas can be summed up and then divided 
by cross sectional area to calculate image processing porosity $\left(R_{I}\right)$. Certain descriptors such as circularity, convexity, and elongation are also employed for characterizing defect morphology in this study according to:

$$
\begin{aligned}
& \text { Circularity }=4 \pi A / P^{2} \\
& \text { Convexity }=P_{\text {convexhull }} / P \\
& \text { Elongation }=1-\left(L_{\text {minor }} / L_{\text {major }}\right)
\end{aligned}
$$

where $A$ is area, $P$ is actual perimeter, $P_{\text {convexhull }}$ is the convex hull perimeter, $L_{\text {minor }}$ is the minor axis length, and $L_{\text {major }}$ is the major axis length. A perfect circle has a circularity of 1 while an irregular object has a circularity value closer to 0 . Convexity is a measure of the surface roughness of a defect. A smooth shape has a convexity of 1 as the convex hull perimeter is exactly the same as the actual perimeter. A very irregular object has a convexity closer to 0 as the actual perimeter is greater than the convex hull perimeter due to the fine surface features. The major axis length of the defective area refers to the length of the major axis of the ellipse that has the same normalized second central moments as the region. Similarly, the minor axis length specifies the length of the minor axis. A shape symmetrical in all axes such as a circle or square will have an elongation value of 0 whereas shapes with large aspect ratios will have an elongation closer to 1 .

\subsection{Results and Discussion}

Statistical distributions of the morphological descriptors of defects in each cross section are illustrated by histograms. Characteristics of defect morphology are analyzed and compared according to these distributions. Melt pool variation is discussed to explain defect formation. 


\subsubsection{Characterization of SLM Defects}

\subsubsection{Defect Morphology of Zone II Defects}

When laser power, hatch spacing and layer thickness are constant, increasing the scan speed will lower the energy density during SLM (Thijs et al, 2010). Figure 3.5 shows horizontal and vertical cross sections of Ti-6Al-4V specimens built using Zone II marginal parameters, with scan speed ranging from $360 \mathrm{~mm} / \mathrm{s}$ to $600 \mathrm{~mm} / \mathrm{s}$.
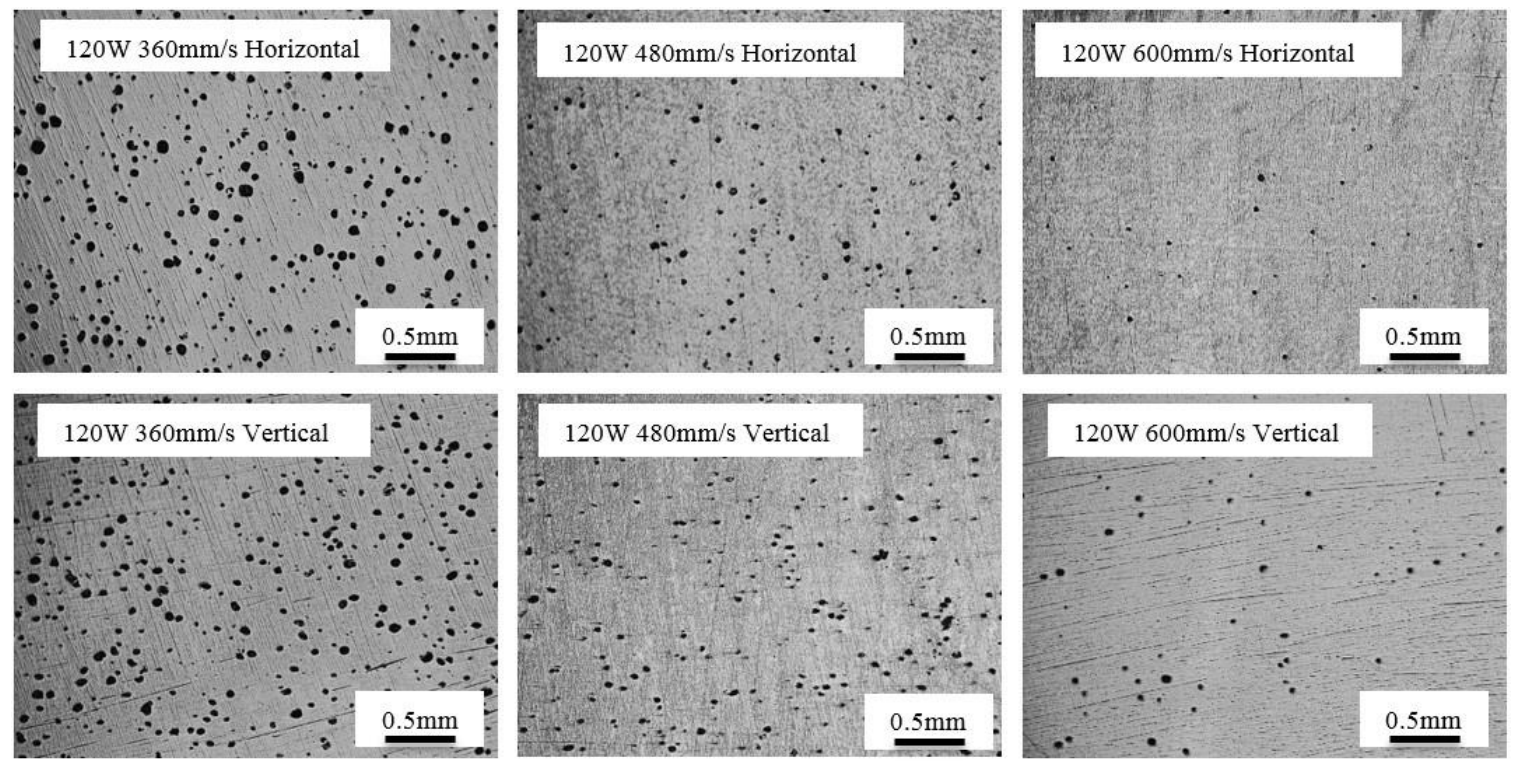

Figure 3.5. Cross section of SLM-produced Zone II specimens.

It can be seen that the defect amount decreases gradually when increasing the scan speed to the fully dense melting zone. Defects are randomly spread on the horizontal and vertical cross sections. The defect dimension is also reduced with decreasing porosity. This trend is verified by the histograms in Figure 3.6 (a) by the defects' area and their frequency of occurrence. Large defects only appears with higher porosity. Zone II defects are typically in round shape. Their morphology is well interpreted by the histograms of circularity, convexity, and elongation, as shown in Figure 3.6 (b), (c) and (d). 
As shown in Figure 3.6 (b), a fitting curve shows a Weibull distribution with right skewness for defect circularity. Most Zone II defects have circularity values close to 1 , which means they are circular or near-circular. Moreover, all Zone II defects have a convexity value larger than 0.5 , as shown in Figure 3.6 (c).

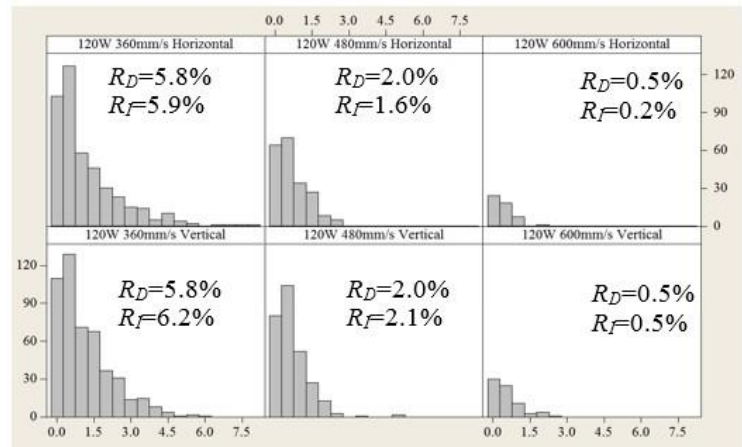

(a) Histograms of Defect Area $\left(\times 10^{3} \mu \mathrm{m}^{2}\right)$

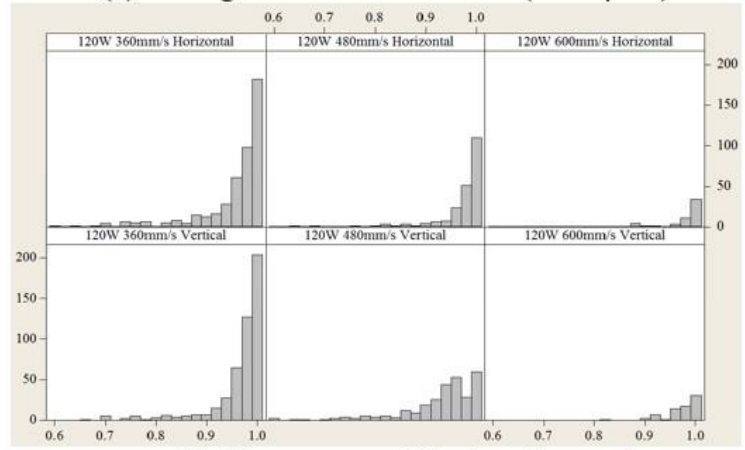

(c) Histograms of Defect Convexity

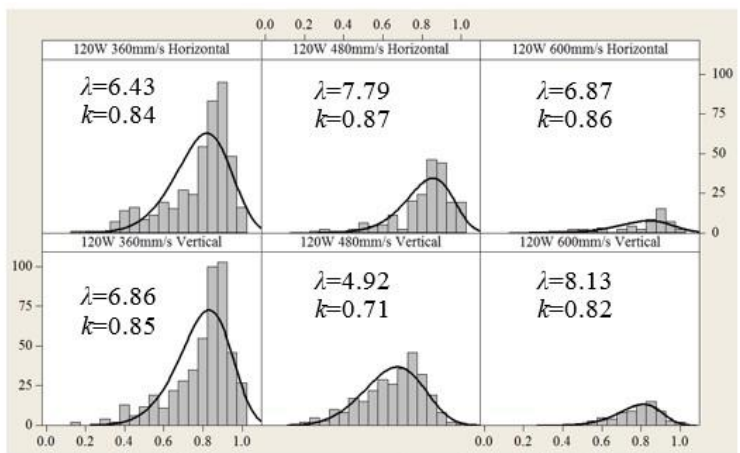

(b) Histograms of Defect Circularity

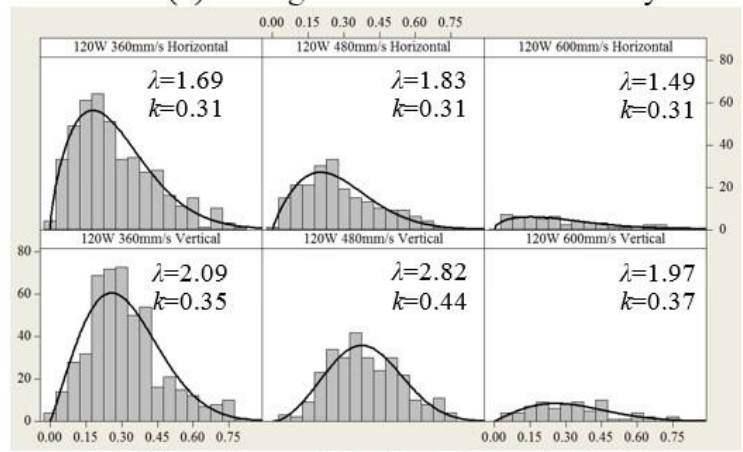

(d) Histograms of Defect Elongation

Figure 3.6. Distribution of defect morphology of SLM-produced Zone II specimen.

A circular contour is a typical convex shape. Thus, it is easy to understand why most Zone II defects have a convexity value close to 1. As shown in Figure 3.6 (d), the fitting curve of Zone II defect elongation also shows a Weibull distribution, but with different shape compared to circularity. Their left skewness indicates that the minor axis length is close to the major axis length, but not completely equal. According to the circularity and elongation distribution, it can be concluded that most defects have an elliptical contour for Zone II specimens. The shape parameter $\lambda$ and scale parameter $k$ of 
the Weibull fitting curve show slight difference between horizontal and vertical cross sections. This implies that the elliptical contours of Zone II defects are distinguishable based upon orientation and thus the material has porosity anisotropy. The $\lambda$ value of Zone II defect elongation is closer to 0 in the horizontal direction than in the vertical direction. This demonstrates that defects are more circular in the plane parallel to scan surface.

\subsubsection{Defect Morphology of Zone III Defects}

Cross sections of Ti-6Al-4V specimens built by Zone III marginal parameters are shown in Figure 3.7. Laser power is kept constant at $80 \mathrm{~W}$. Scan speed ranges from $840 \mathrm{~mm} / \mathrm{s}$ to $1320 \mathrm{~mm} / \mathrm{s}$. When the scan speed is far away from Zone I, the energy density will be lowered which results in incomplete melting during the SLM process. It is noted that the porosity increased with increasing scan speed.
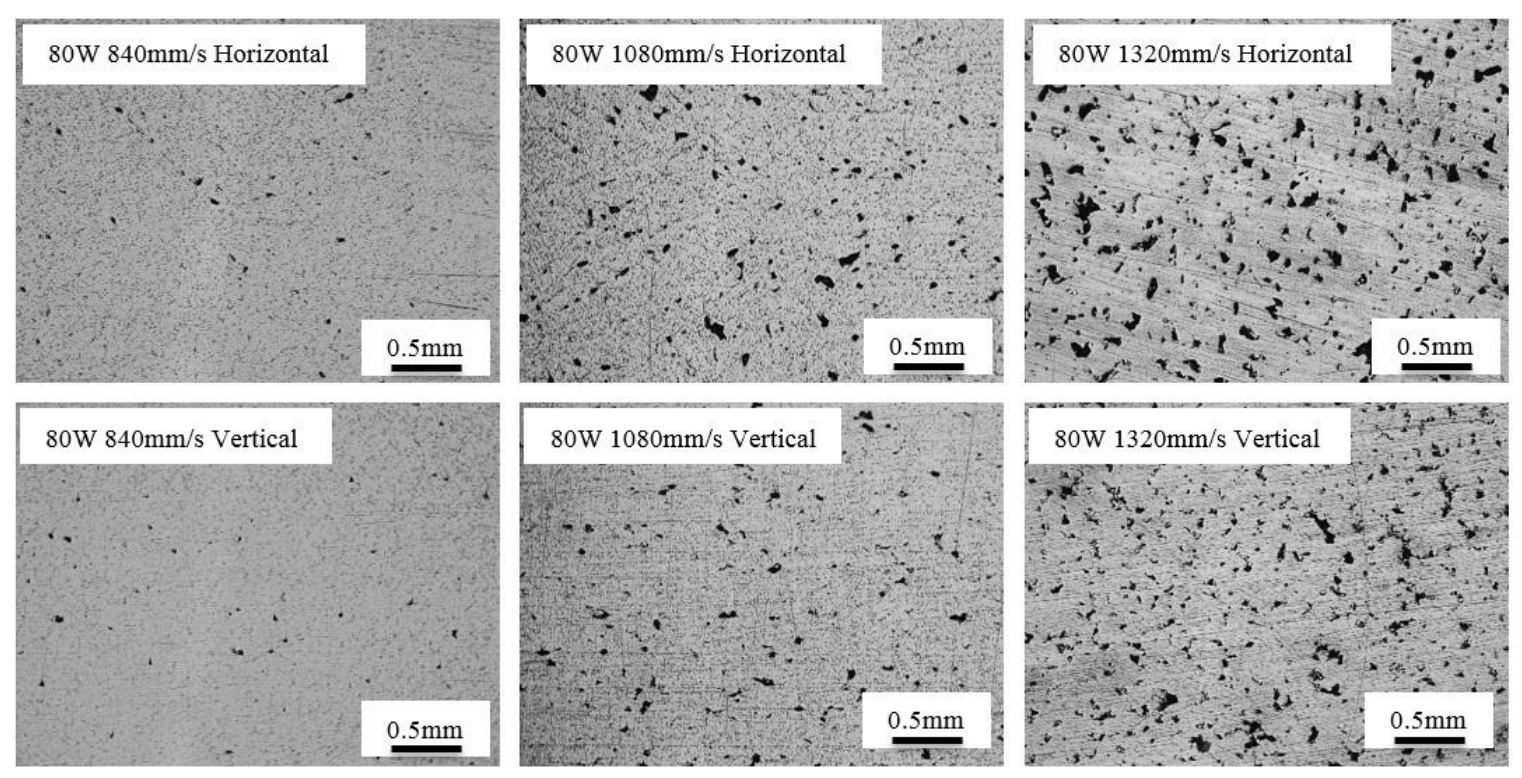

Figure 3.7. Cross section of SLM-produced Zone III specimens.

Defect dimension increases when increasing scan speed. Similar to Zone II defects, the higher the porosity, the larger the defect dimension. However, unlike Zone II defects, 
Zone III defects are in irregular shape, in both horizontal and vertical cross sections. Large defects appears in Zone III specimens but with a small amount, as shown in Figure 3.8 (a).

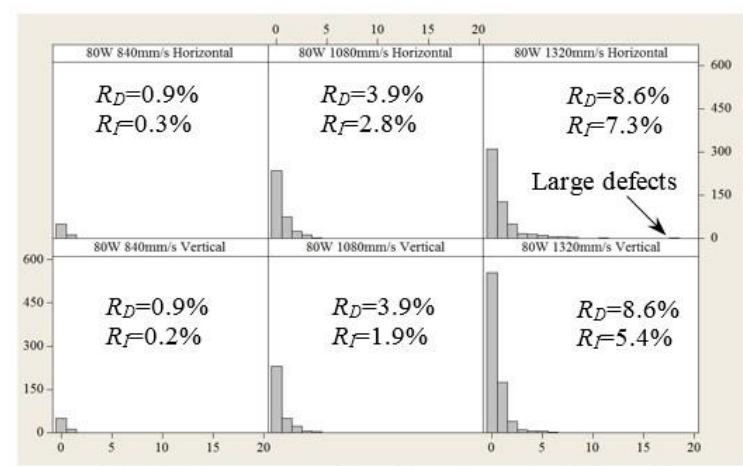

(a) Histograms of Defect Area $\left(\times 10^{3} \mu \mathrm{m}^{2}\right)$

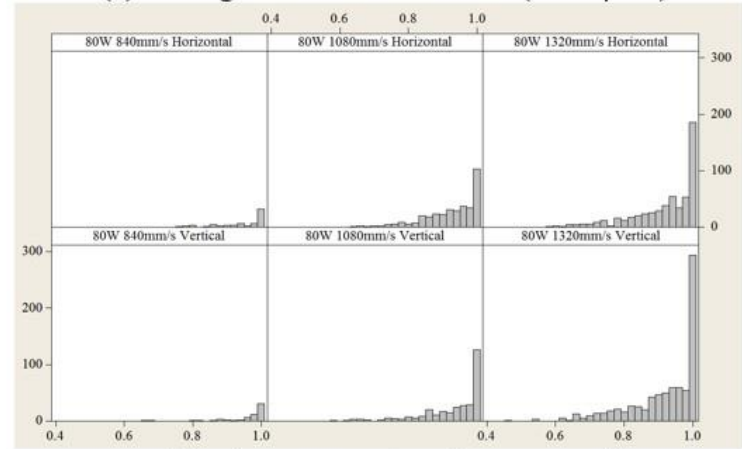

(c) Histograms of Defect Convexity

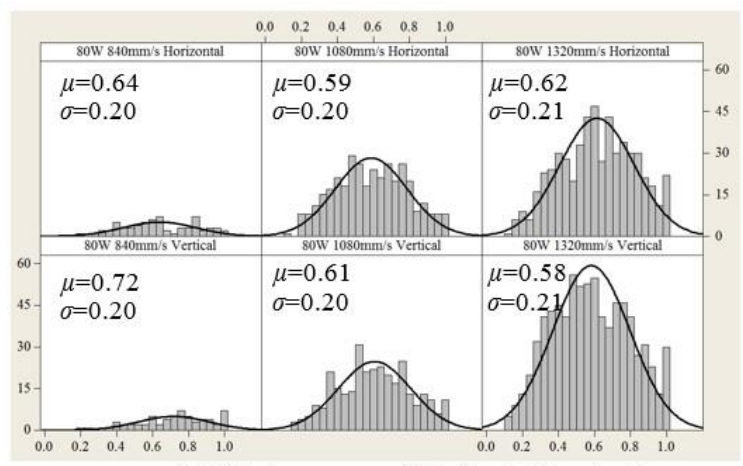

(b) Histograms of Defect Circularity

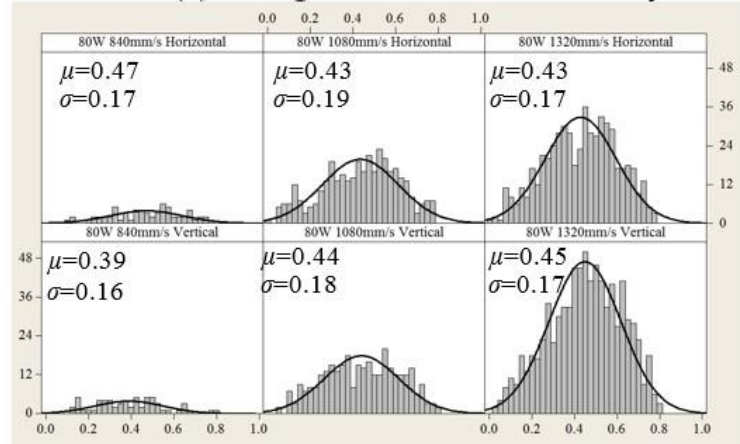

(d) Histograms of Defect Elongation

Figure 3.8. Distribution of defect morphology of SLM-produced Zone III specimen.

The morphology of Zone III defects is different from Zone II defects, according to the distribution fitting curves of circularity and elongation. Figure 3.8 (b) and (d) show that the circularity and elongation are all normally distributed, no matter whether in horizontal or vertical cross sections. The normal distributions of elongation have mean values ranging from 0.39 to 0.47 , with standard deviation from 0.16 to 0.19 . It can be seen that more than $70 \%$ of Zone III defects have an elongation value less than 0.5 . Only $4 \%$ of Zone III defects have elongation value larger than 0.7 , which means a long and narrow contour. As shown in Figure 3.8 (c), some Zone III defects show concave characteristics, which illustrates their irregular shape. 


\subsubsection{Comparison of Defect Morphology in Zone II and Zone III Specimens}

Generally, Zone II and Zone III defects show quite different morphological characteristics, especially in circularity and elongation. In order to further characterize the morphology, specimens with Zone II and Zone III defects are compared in Figure 3.9. Both specimens have similar porosity $(\sim 5.8 \%)$. It is notable that the energy density of marginal parameters $(120 \mathrm{~W} \& 360 \mathrm{~mm} / \mathrm{s})$ in Zone II is higher than that of Zone III marginal parameters $(120 \mathrm{~W} \& 1560 \mathrm{~mm} / \mathrm{s})$.
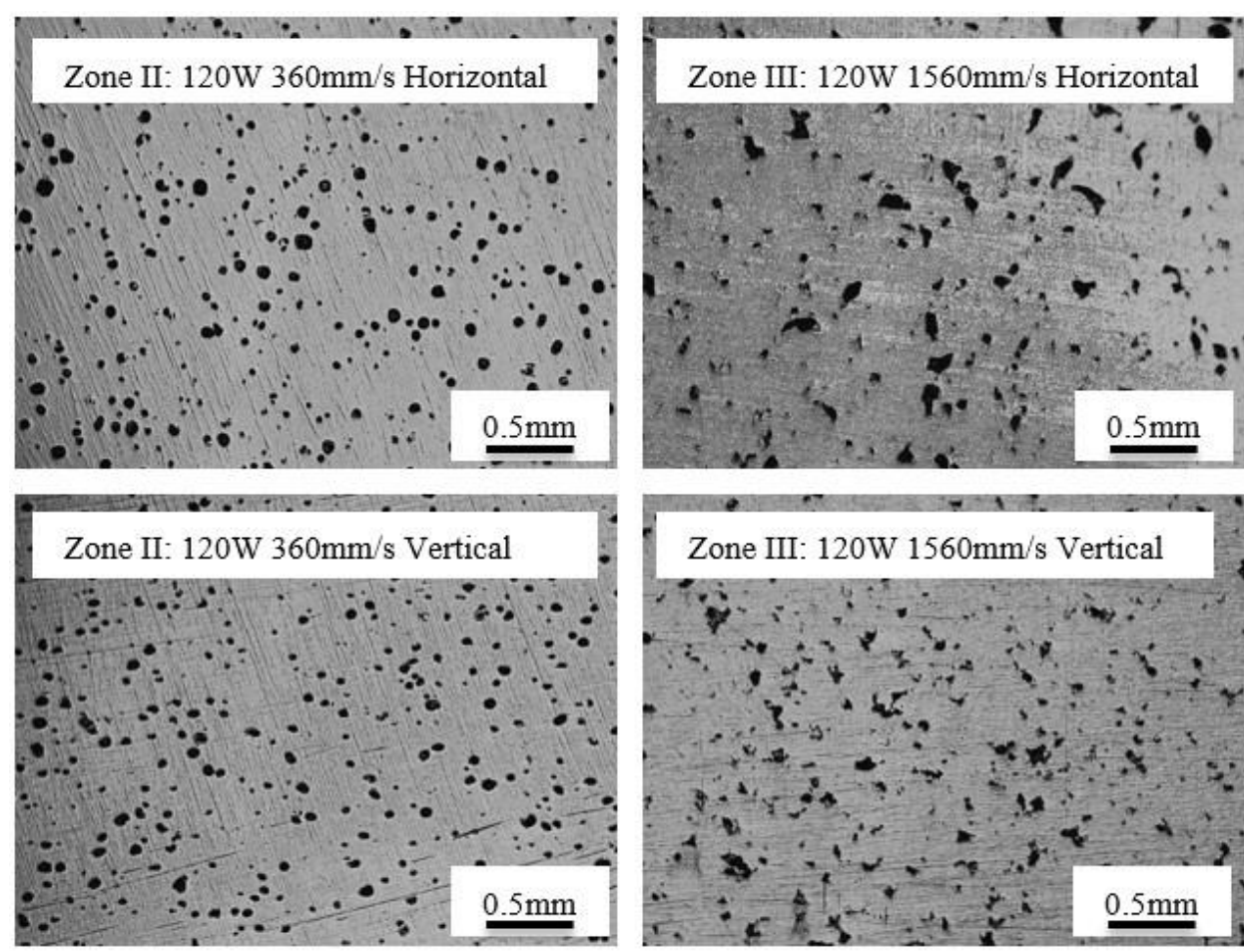

Figure 3.9. Comparison of cross section of SLM-produced specimen.

Most Zone II defects are round in shape, while Zone III defects are large with an irregular shape. From Figure 3.10 (a), it can be seen that most Zone II defects are have an area less than $6 \times 10^{3} \mu \mathrm{m}^{2}$, while some Zone III defects have areas up to $14 \times 10^{3} \mu \mathrm{m}^{2}$. 
Weibull distribution is a proper choice to describe Zone II defect morphology, while Zone III defect morphology can be described using a normal distribution. The Weibull distribution statistically verifies that most Zone II defects are circular contour in both horizontal and vertical cross sections.

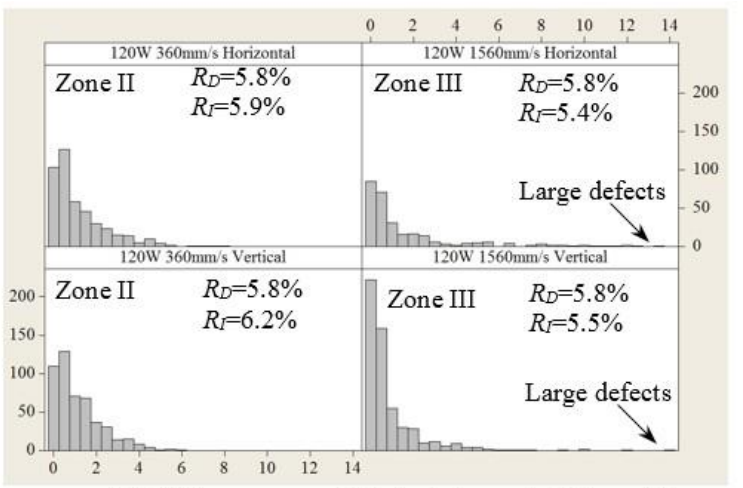

(a) Histograms of Defect Area $\left(\times 10^{3} \mu \mathrm{m}^{2}\right)$

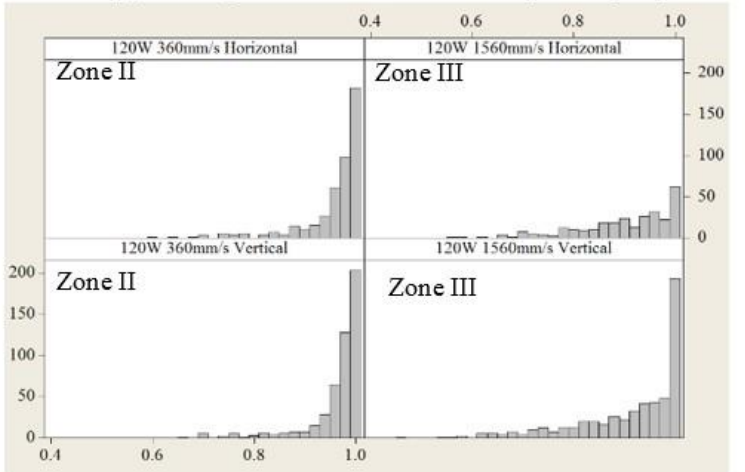

(c) Histograms of Defect Convexity

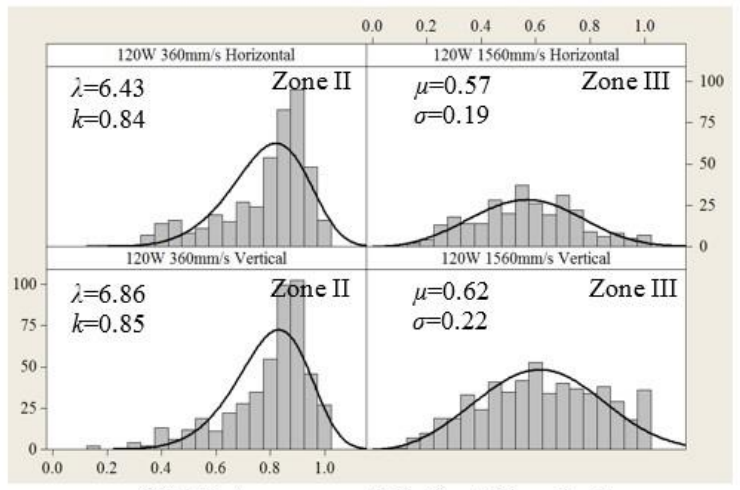

(b) Histograms of Defect Circularity

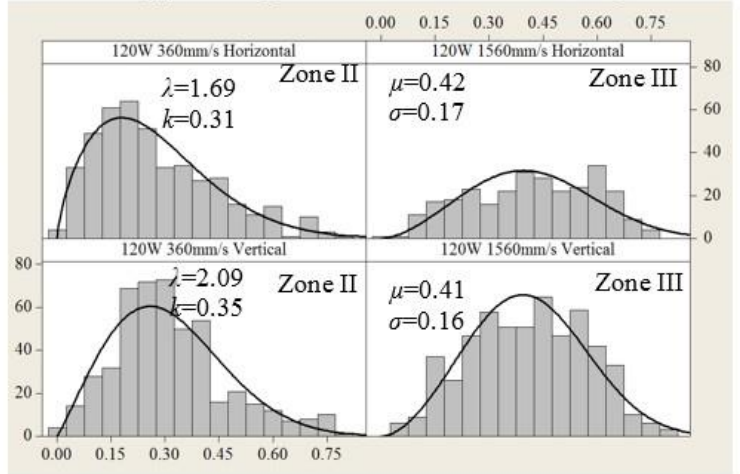

(d) Histograms of Defect Elongation

Figure 3.10. Distribution of SLM Zone II and III defect morphology (porosity $\approx 5.8 \%$ ).

Zone III defects are formed between melt pools or hatch lines due to insufficient energy density. Thus, it makes sense that Zone III defects are spread stochastically with irregular shape. Both circularity and elongation histograms of defects are normally distributed. An SLM Ti-6Al-4V top surface fabricated with marginal parameters is shown in Figure 3.11. 

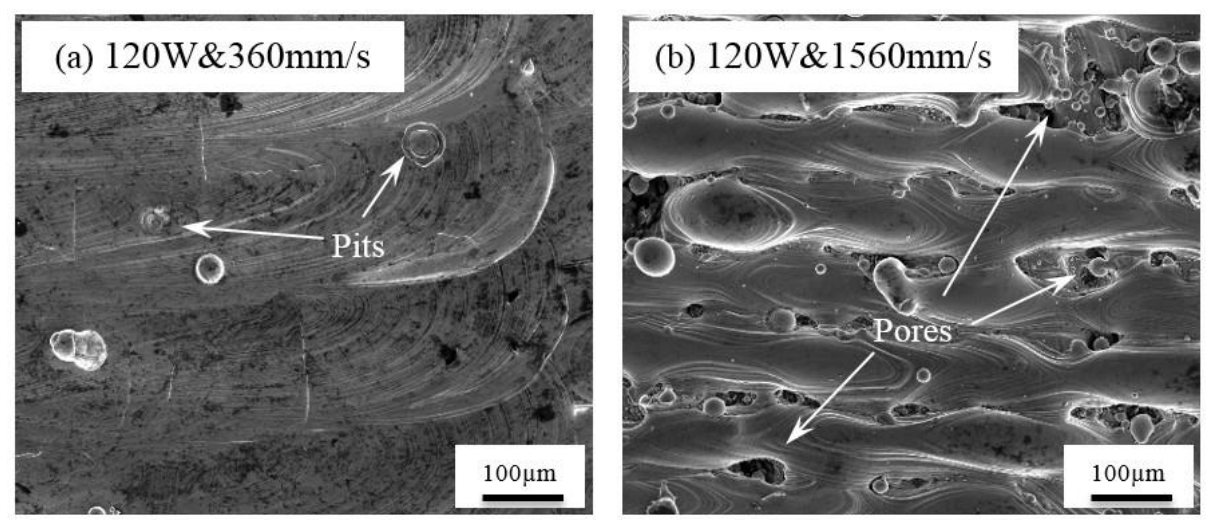

Figure 3.11. Top surfaces of SLM specimens by marginal parameters (a) Zone II; and (b) Zone III.

When Ti-6Al-4V powder was melted using Zone II marginal parameters, the melt pool was extremely enlarged. Large melt pool results in a large overlap between hatch lines. However, no voids or defects were directly formed by the large melt pool and highly overlapped hatch lines. Spherical pits, as shown in Figure 3.11 (a), are believed to cause spherical defects in Zone II specimens. These pits result from the recoating blade scraping particles, which are solidified from the ejected molten materials on the surface. This phenomenon is dramatic because Zone II marginal parameters melt the powder with high energy density. By contrast, the Zone III voids or pores directly generated on the top surface, as shown in Figure 3.11 (b), are entrapped by new recoated and melted layers.

\subsubsection{Characterization of EBM Defects}

EBM defects resulted from the increased line offset and focus offset values. Energy density is decreased due to these marginal parameters. Compared to laser melting, EBM defects are formed similarly to Zone III defects in SLM. In this section, the morphology of line offset defects and focus offset defects are analyzed. 


\subsubsection{Defect Morphology of Line Offset $(L O)$ Defects}

Porosity dramatically increases when the line offset value is larger than $0.18 \mathrm{~mm}$, according to the porosity distribution curve in Figure 3.2. Thus, Figure 3.12 shows the cross sections of specimens at specific line offsets of $0.18 \mathrm{~mm}, 0.22 \mathrm{~mm}, 0.26 \mathrm{~mm}$, and $0.30 \mathrm{~mm}$.
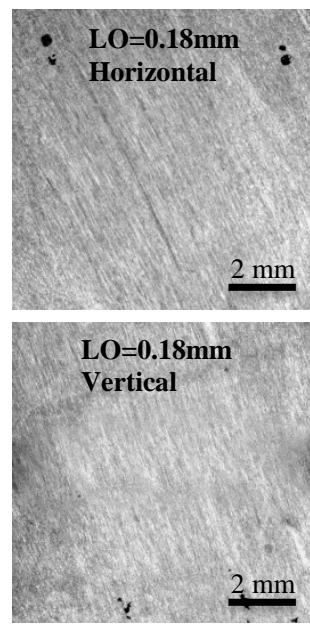
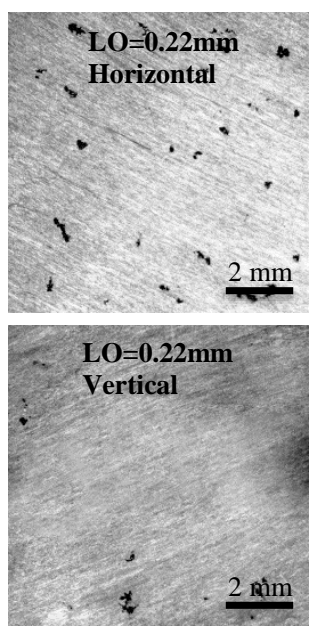
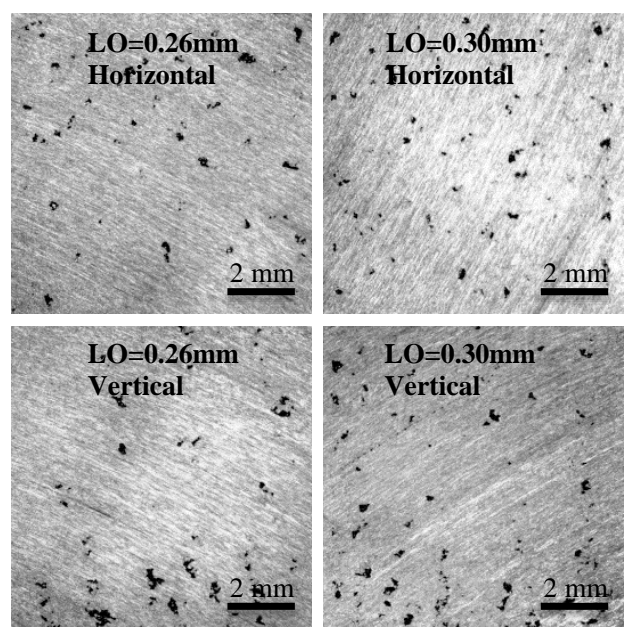

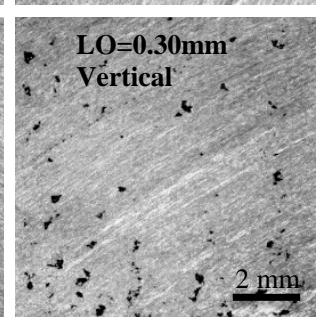

Figure 3.12. Cross sections of EBM-produced specimen at various line offset values.

$L O$ defects are spread on the cross sections of Ti-6Al-4V specimens unequally. Large defects can be observed directly with bare eyes. It can be seen that, when line offset is $0.18 \mathrm{~mm}$ there are only a few defects showing on the cross sections. However, as line offset increases, the defect amount increases correspondingly. As shown in Figure 3.13 (a), most $L O$ defect areas are smaller than $30 \times 10^{3} \mu \mathrm{m}^{2}$. Large defects are rare, but can have extremely large area. A Weibull fitting curve can be used to describe the distribution of the histograms of $L O$ defect circularity. But the curve characteristics are very different from that of SLM Zone II or Zone III defects. From Figure 3.13 (b), it is noted that all the fitting curves show left skewness, which means no defects have a circular contour. Thus, most EBM defects are very irregular. 


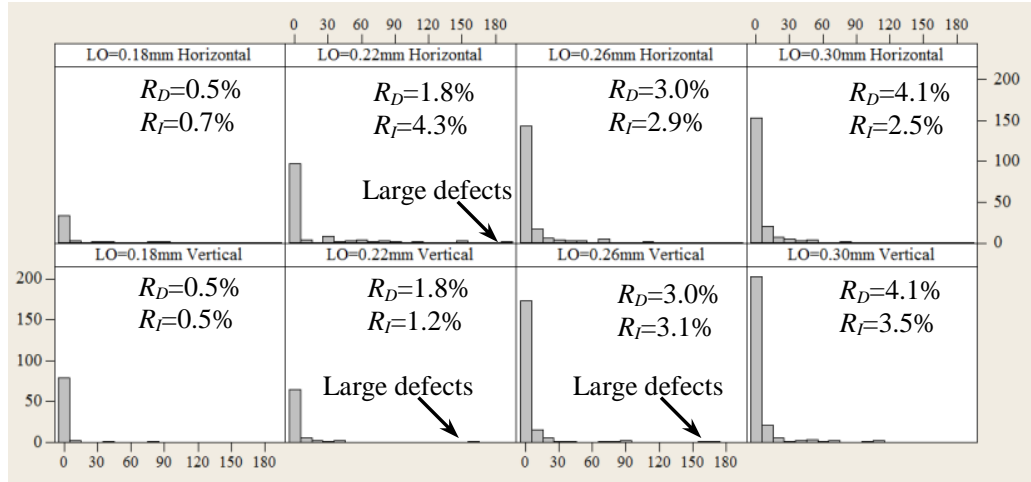

(a) Histograms of Defect Area $\left(\times 10^{3} \mu \mathrm{m}^{2}\right)$

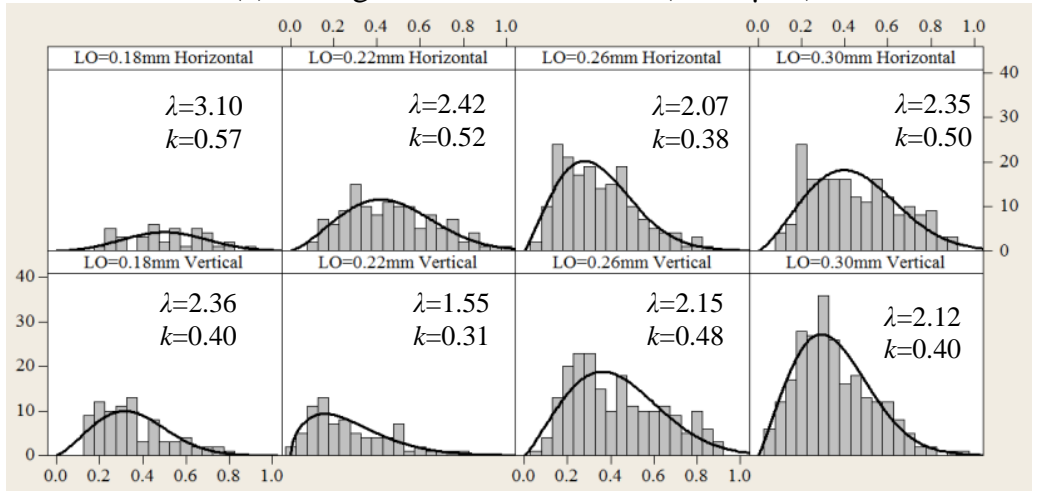

(b) Histograms of Defect Circularity

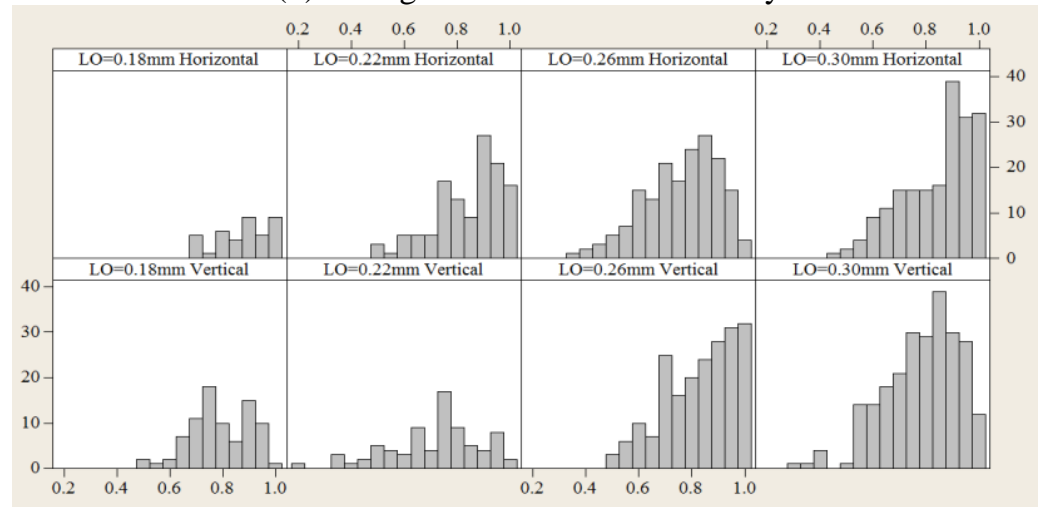

(c) Histograms of Defect Convexity

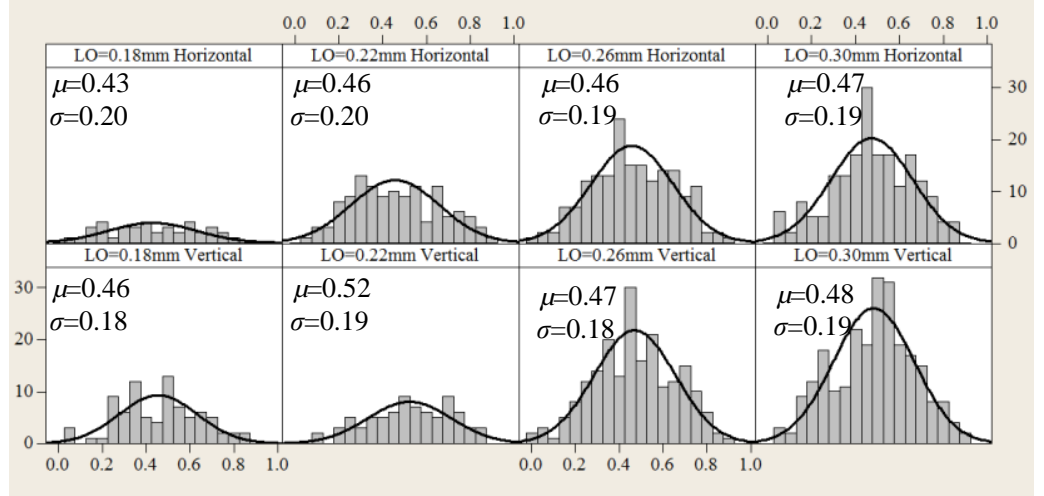

(d) Histograms of Defect Elongation

Figure 3.13. Distributions of $L O$ defect morphology of EBM-produced specimens. 
Defect convexity, shown in Figure 3.13 (c), verifies the irregularity of $L O$ defects. Although many defects have a convexity value close to 1 , there are a large amount of defects with small convexity values. This indicates that these defects have concave contour, as shown on the cross sections. Elongation of $L O$ defects can be described by normal distribution fitting curves. All fitting curves, as shown in Figure 3.13 (d), have mean values close to 0.50 , and standard deviation close to 0.20 . This is similar to Zone III defects of SLM specimens. Most $L O$ defects (about $84 \%$ ) have an elongation value less than 0.7 .

\subsubsection{Defect Morphology of Focus Offset $(F O)$ Defects}

Cross sections of Ti-6Al-4V specimens with $F O$ defects are shown in Figure 3.14. Large defects can also be observed with the naked eye from sectioned surface. Some defects are successive on the vertical cross sections. These defects are spread vertically from the bottom to the top surface. It is believed that an increased focus offset value generates an extended melt pool. But the reduced melting depth creates an unstable melt pool due to the underneath un-melted powder. Voids and pores are generated due to the instability of the melt pool. This means that $F O$ defects are often created above the voids or pores of a previous layer. Thus, successive defects grows as shown in the vertical cross sections.

As for the morphology of $F O$ defects, it can be seen, in Figure 3.15 (a), that most defects are small, with an area less than $15 \times 10^{3} \mu \mathrm{m}^{2}$. Only a few large defects were found. The circularity distribution of $F O$ defects can also be described by Weibull fitting curves, as shown in Figure 3.15 (b). Few defects show circular contour according to the left skewness of the distribution characteristics. Figure 3.15 (c) shows the convexity of $F O$ 
defects. Most defects have a concave contour. It is predictable that narrow and long defects exists in the specimens. A normal distribution can be used to describe the elongation of $F O$ defects. Its statistical characteristics are similar to that of $L O$ defects.
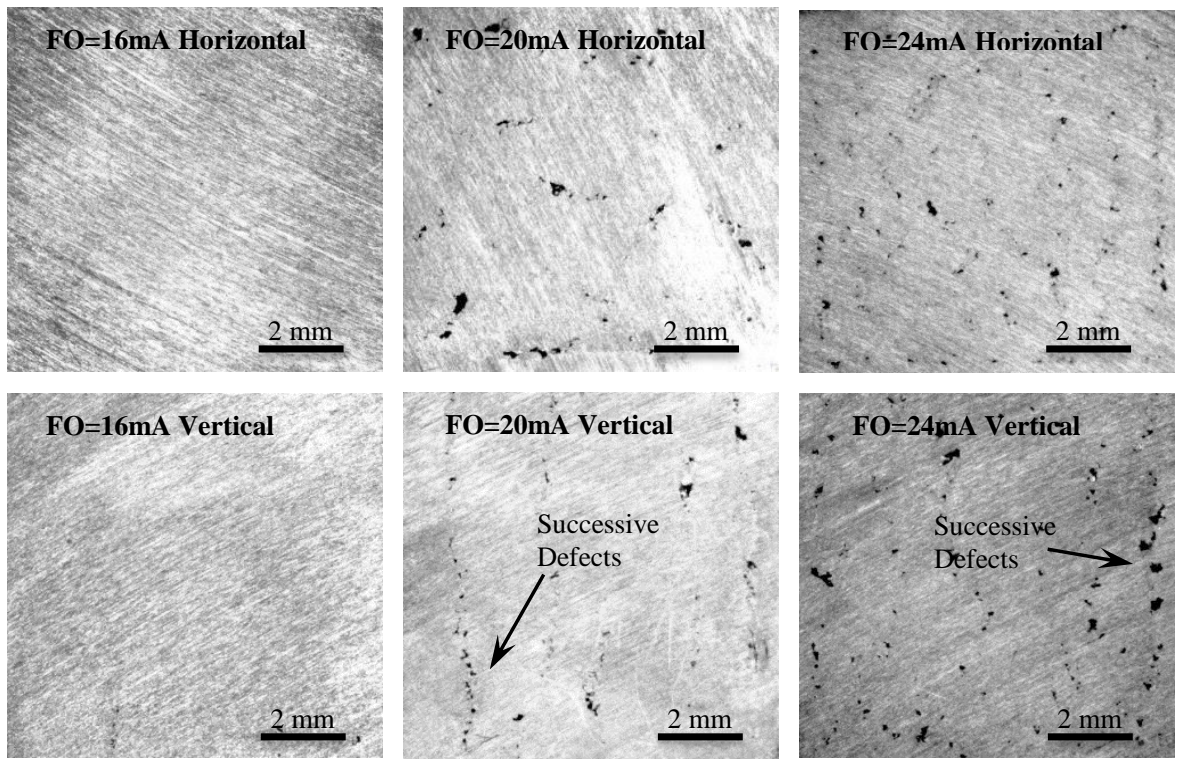

Figure 3.14 Cross sections of EBM-produced specimen at various focus offset values.

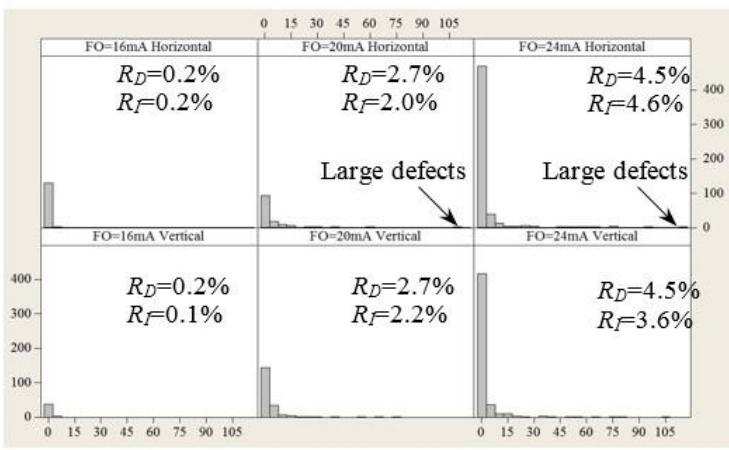

(a) Histograms of Defect Area $\left(\times 10^{3} \mu \mathrm{m}^{2}\right)$

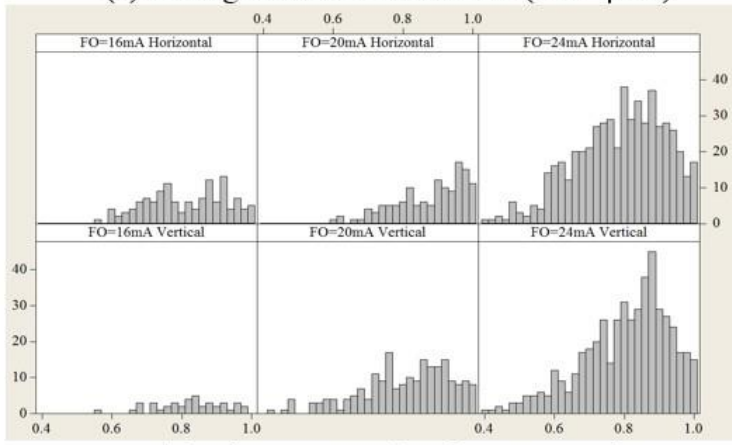

(c) Histograms of Defect Convexity

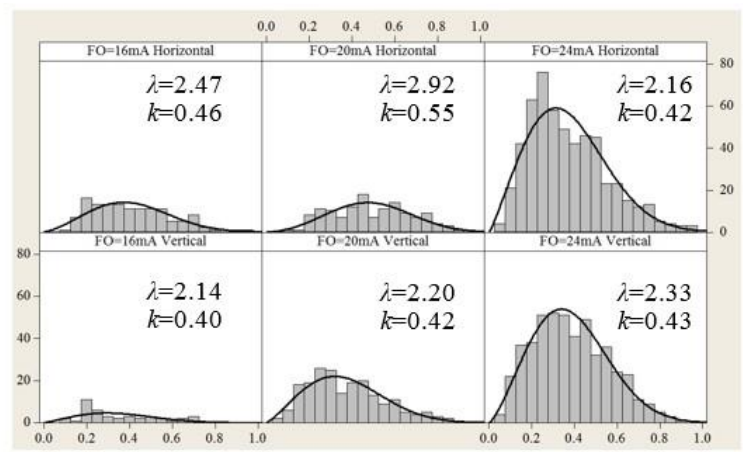

(b) Histograms of Defect Circularity

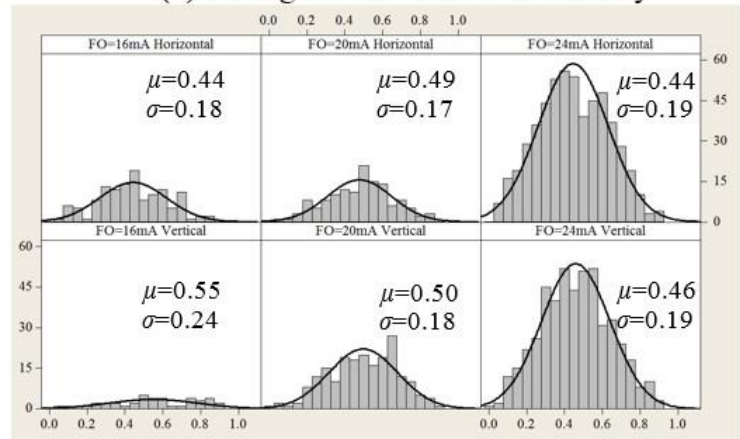

(d) Histograms of Defect Elongation

Figure 3.15. Distribution of $F O$ defect morphology of EBM-produced specimens. 


\subsubsection{Comparison between $L O$ Defects and $F O$ Defects}

The morphological characteristics of $L O$ defects and $F O$ defects are similar, based on the defect area, circularity, convexity, and elongation distribution of histograms. Thus, it can be determined that the morphology of $L O$ defects and $F O$ defects are the same. The only difference is the spreading characteristics of defects in the specimens. From the cross section of EBM specimens, it can be seen that $L O$ defects are randomly spread on the horizontal and vertical planes. It is hard to say any of defects are correlated with each other. However, successive $F O$ defects are aligned in the vertical plane. The defects resulting from an increased focus offset more easily lead to the growing of defective region.

The formation of EBM defects can be further explained by the melt pool characteristics on the top surface. The melt pools are isolated from each other due to the increased line offset. Molten powder is easily fused together causing voids, as shown in Figure 3.16 (a). Although melt pools are highly overlapped when increasing focus offset, a certain amount of powder underneath is not melted due to the shallow depth of the melt pools. The un-melted powder has an impact on the upper surface where voids are formed, as shown in Figure 3.16 (b).
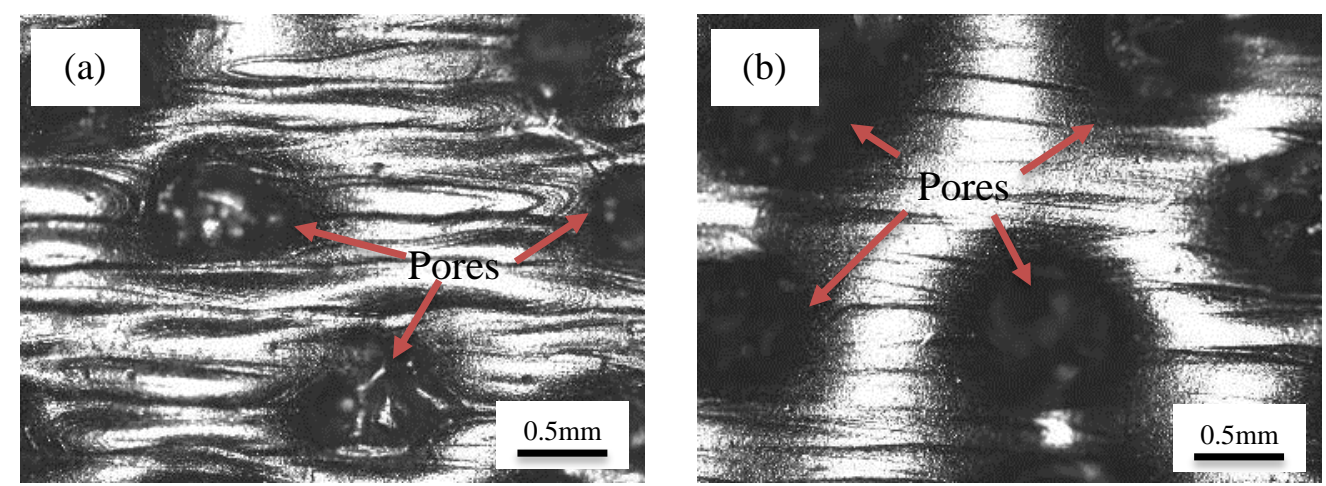

Figure 3.16. Top surface of EBM specimens (a) $L O=0.26 \mathrm{~mm}$ and (b) $F O=20 \mathrm{~mA}$. 


\subsubsection{Image Processing Porosity $R_{I}$}

The porosity $R_{D}$ is obtained by measuring the specimen's relative density. It is considered an accurate method for estimating the porosity of SLM and EBM materials. $R_{I}$ is used as a reference for estimating a specimen's porosity according to the ratio of defective area to the overall area of each cross section. It is notable that $R_{I}$ is very close to $R_{D}$. It can be inferred that, if enough cross sections are processed, the image processing porosity $R_{I}$ should be a feasible way of estimating the actual porosity of specimens. Although $R_{I}$ has to be obtained from destructive characterization techniques, it enables the avoidance of lacquering or oil impregnation, which must be applied to the surface of porous parts to avoid penetration of fluid when using Archimedes method. Thus, image processing porosity can be considered an alternative way of estimating porosity of additively manufactured specimens.

\subsection{Conclusion}

Defect morphology of SLM and EBM specimens were studied using image processing to investigate defect area, porosity and other morphological characteristics such as circularity, convexity, and elongation. It can be concluded that higher porosity is usually accompanied with larger defect dimensions in both SLM and EBM specimens. Defect morphology can be correlated with melt pool characteristics.

Zone II defects formed by high energy density are distributed in SLM specimens and have a spherical shape. Porosity is a result of mechanically scraping the solidified particles, which are ejected from melt pool. A Weibull distribution can be used to describe histograms of the circularity and elongation value of Zone II defects. Zone III defects, 
which are formed by low energy density levels, are irregular in shape in SLM. Defects are directly generated due to the insufficient energy for complete melting. Porosity is entrapped when new recoated and melted layers are applied over top. The histograms of circularity and elongation value of Zone III defects are normally distributed.

Line offset defects and focus offset defects in EBM are all formed due to a reduced energy density, similarly to Zone III defects in SLM. Their irregularity can be attributed to the large pores formed on the top surface. There are nearly no circular defects in EBM specimens according to the circularity distribution. A certain amount of defects show concave characteristics.

Image processing porosity $R_{I}$ can reflect the approximate porosity of porous parts. Thus, image processing can be a feasible way of estimating porosity if cross section images are well processed. 


\section{CHAPTER 4}

\section{MICRO-CT EVALUATION OF STOCHASTIC DEFECTS FABRICATED}

\section{BY SLM AND EBM ${ }^{4}$}

\subsection{Introduction}

Additive Manufacturing (AM) is able to produce parts directly from a 3D CAD model, usually layer upon layer. In AM, material accumulation via material fusion only in prescribed regions of the build area enables complex parts to be easily made. In recent years, Powder Bed Fusion (PBF) based AM processes, such as Selective Laser Melting (SLM) and Electron Beam Melting (EBM) (Gibson et al, 2009), have been developed so that functional parts can be directly fabricated from pre-alloyed powders (such as stainless steel, cobalt chromium, titanium, etc.) without traditional casting or molding processes. PBF-produced Ti-6Al-4V parts are widely adopted in aerospace, biomedical and industrial fields due to their inherent properties of fracture resistance, fatigue behavior, corrosion resistance and biocompatibility (Soboyejo et al, 2006).

During SLM processing, a CAD model is first "sliced" into layers of a prescribed thickness and then uploaded to the SLM machine. A focused laser beam is used to melt metallic powder into a solid part according to the contour information of the sliced layers.

\footnotetext{
${ }^{4}$ This chapter is prepared as a journal paper for NDT \& E International. All permissions to use this paper as a part of this dissertation are contained in Appendix D.
} 
Inside the building chamber, there is a material dispensing platform and a build platform along with a recoater blade used to add new powder over the build platform, as shown in Figure 4.1. Parts are built up additively layer by layer. The CAD model and slicing data for the EBM process is similar to the SLM process. But, instead of a laser beam, an electron beam is generated by a tungsten filament in the electron beam gun as shown in Figure 4.2.

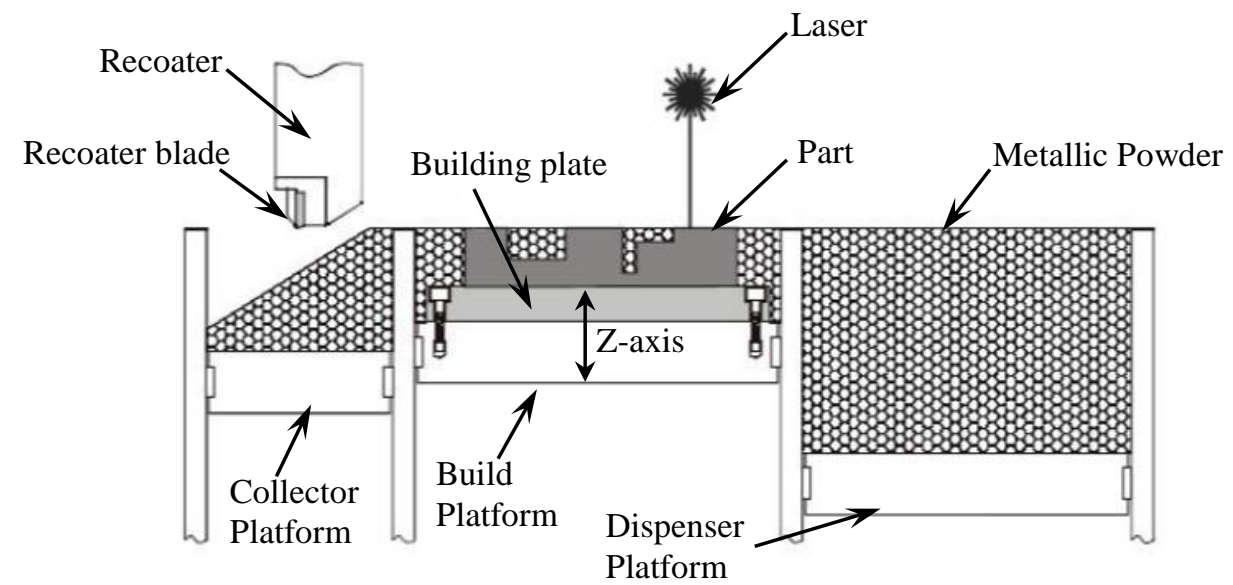

Figure 4.1. Schematic of SLM process (source: EOS basic training manual).

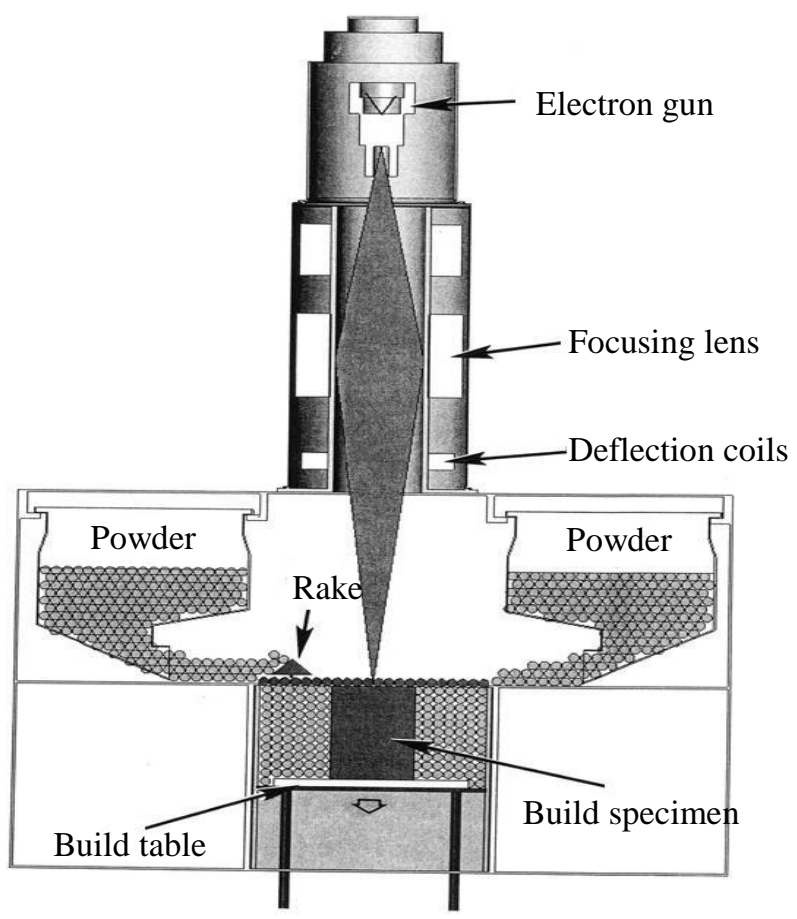

Figure 4.2. Schematic of EBM process (Murr et al, 2009a). 
The concentrated electron beam is accelerated to a tremendously high speed for achieving high energy electrons. When these electrons penetrate the powder surface and further into the powder grains, their velocity reduces. By doing this, their kinetic energy converts into thermal energy and the metal powder reaches its melting temperature (Hiemenz, 2007). Electromagnetic lenses are employed to control the electron beam preheating the building platform and melting the powder at certain area, compared to scanning galvanometers in the SLM process.

Properties of additively manufactured parts are dramatically influenced by process parameters, scanning strategy and raw material characteristics. A great many investigations have been conducted and focused on process parameter optimization for fully dense parts. Usually AM parts are evaluated using the Archimedes method or destructive characterization method. Although these methods are not efficient, they are effective for estimating density and porosity (Spierings et al, 2011). In order to accurately display 3D internal structure, some researchers employed Computed Tomography (CT) for analyzing defects and complex struts made by SLM or EBM (Spierings et al, 2011; Parthasarathy et al, 2010; Van Bael et al, 2011). However, few investigations were carried out on detecting stochastic defects generated by near-optimized parameters using CT. Thus, this chapter aims to study the capability of micro-CT for detecting stochastic defects in SLM and EBM specimens of various porosities, and to discuss the possibility for effective porosity determination through CT scanning results. Titanium alloy Ti-6Al-4V is primarily used in this study.

\subsection{Theory of Micro CT}


Micro CT uses the penetrating abilities of X-rays to examine the internal defects of objects (Carvalho et al, 2008). An X-ray source is used to send radiation through a part. Material discontinuities such as voids or changes in contour affect the degree of radiation absorption. The unabsorbed radiation that passes through the part is recorded to indicate the internal characteristics such as voids, pores or cracks.

The intensity of the X-ray is modified according to the internal structure of the specimen to generate CT slices. Each CT slice is subdivided into a 3D matrix of volumetric elements (voxels). The intensity of radiation is measured by a detector. In a slice, the average attenuation value within each voxel is calculated from the intensity readings and assigned as a CT number. Generally, grayscale intensity in the CT slice image is used as the CT number. Denser material is rendered with a higher grayscale value (bright region) in the image, while voids or defects are displayed as dark. However, the image grayscale cannot represent the actual density information of the specimen. Image brightness and contrast are also influenced by the CT setup. Gradient grayscale distribution is even shown for the same scenario with a consistent configuration.

\subsection{Experimental Procedures}

\subsubsection{Specimen Fabrication}

SLM- and EBM-produced specimens can be fabricated using marginal parameters to generate stochastic defects with porosity. Depending on the energy input, SLM processing parameters can be subdivided into several melting zones, such as "fully dense parameters" (Zone I), "over melting parameters" (Zone II), and "incomplete melting parameters" (Zone III). Zone I parameters can be considered as optimum because they are 
capable of fabricating fully dense SLM part. Stochastic defects can be generated by Zone II or III parameters due to the excessive or insufficient energy input. Thus Zone II and III parameters are all marginal parameters. EBM marginal parameters are similar to the Zone III marginal parameters of SLM. Defects are attributed to insufficient energy density. An EOS M270 Direct Metal Laser Sintering (DMLS) system was utilized for building SLM specimens with Raymor Ti-6Al-4V powder (Grade 23). Scan speed was varied with constant laser power $(120 \mathrm{~W})$, hatch spacing $(0.1 \mathrm{~mm})$ and layer thickness $(0.03 \mathrm{~mm})$ to conform to a Zone II or III marginal parameter combination. Arcam Titanium Ti-6Al-4V ELI powder was used for building EBM specimens (layer thickness $0.05 \mathrm{~mm}$ ) with an Arcam S400 EBM system. Line offset and focus offset are increased separately for generating stochastic defects. Other processing parameters were kept constant. Cubical specimens with side dimensions of $10 \mathrm{~mm}$ were built to evaluate the capability of micro CT. Each specimen's density was measured using the Archimedes method to estimate porosity $\left(R_{D}\right)$. The marginal parameters and corresponding porosity are shown in Table 4.1 .

Table 4.1 Marginal Parameters of SLM and EBM

\begin{tabular}{|c|c|c|c|c|}
\hline PBF & \multicolumn{2}{|c|}{ Marginal Parameters } & $R_{D}$ & Nomenclature \\
\hline \multirow{6}{*}{ SLM } & \multirow{3}{*}{$\begin{array}{l}\text { Over melting } \\
\quad(\text { Zone II })\end{array}$} & $V=360 \mathrm{~mm} / \mathrm{s}$ & $6.0 \%$ & \multirow{12}{*}{$\begin{array}{l}V=\text { scan speed } \\
L O=\text { line offset } \\
F O=\text { focus offset }\end{array}$} \\
\hline & & $V=480 \mathrm{~mm} / \mathrm{s}$ & $2.0 \%$ & \\
\hline & & $V=600 \mathrm{~mm} / \mathrm{s}$ & $0.5 \%$ & \\
\hline & \multirow{3}{*}{$\begin{array}{l}\text { Incomplete } \\
\text { melting } \\
\text { (Zone III) }\end{array}$} & $V=1200 \mathrm{~mm} / \mathrm{s}$ & $0.1 \%$ & \\
\hline & & $V=1320 \mathrm{~mm} / \mathrm{s}$ & $2.0 \%$ & \\
\hline & & $V=1560 \mathrm{~mm} / \mathrm{s}$ & $6.0 \%$ & \\
\hline \multirow{6}{*}{ EBM } & \multirow{3}{*}{ Line Offset } & $L O=0.18 \mathrm{~mm}$ & $0.7 \%$ & \\
\hline & & $L O=0.24 \mathrm{~mm}$ & $2.0 \%$ & \\
\hline & & $L O=0.30 \mathrm{~mm}$ & $4.0 \%$ & \\
\hline & \multirow{3}{*}{ Focus Offset } & $F O=16 \mathrm{~mA}$ & $0.3 \%$ & \\
\hline & & $F O=20 \mathrm{~mA}$ & $3.0 \%$ & \\
\hline & & $F O=24 \mathrm{~mA}$ & $4.5 \%$ & \\
\hline
\end{tabular}




\subsubsection{Defects Inspection by Micro CT}

CT scanning was carried out using a micro-CT scanner (Model ACTIS 200/225 Ffi-HR CT/DR system. BIR Inc., Chicago, IL with built-in X-ray system FXE 225.20, Fein Focus U.S.A). As shown in Figure 4.3, the scanner consists of a turnable stage, an X-ray source, an image intensifier, and a CCD camera. Different from regular clinical CT, the micro-CT scanner rotates a specimen on the stage with a fixed X-ray source (voltage 135 $\mathrm{KeV}$ and current $0.059 \mathrm{~mA}$ ) and an image intensifier. The scanner's resolution depends on the physical position of the specimen. In this study, each scan consists of 107 slices. Slices were created $7 \mu \mathrm{m}$ apart, parallel to the building plane of specimens. The voxel in these slices is $7 \times 7 \times 7 \mu \mathrm{m}$. Three dimensional reconstruction was also performed with slices using VGStudio MAX software package (Version 1.2.1) for visualization and analysis.

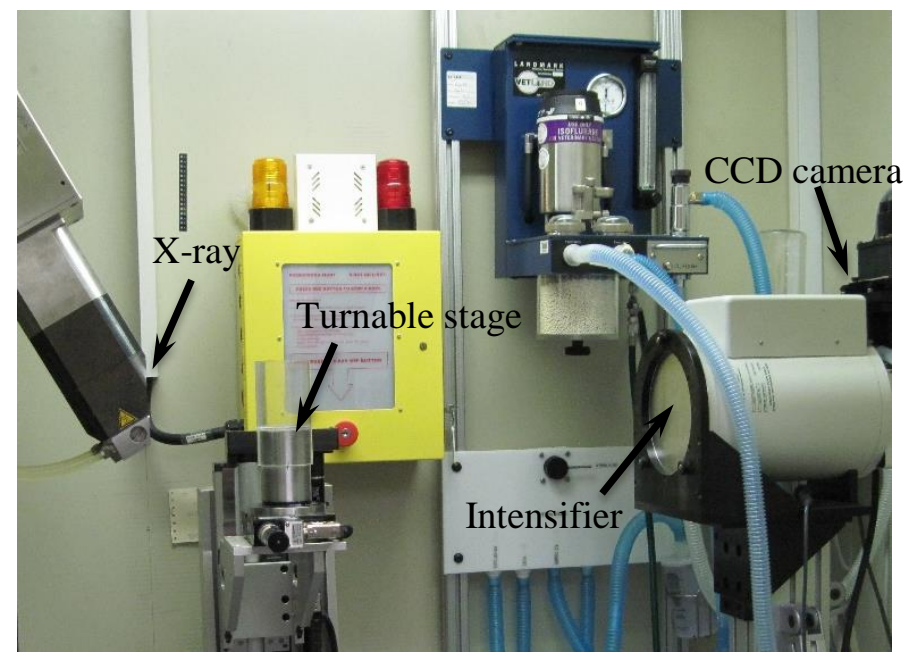

Figure 4.3 Micro CT scanner.

4.4 Results and Discussion

\subsubsection{Characteristics of SLM specimens}

During laser melting of titanium powder, Zone II marginal parameters, which are related to a high energy density, usually cause a complex microscopic or macroscopic 
transport phenomenon with the molten metal. Energy density refers to the average applied energy per volume of material during scanning of one layer (Thijs et al, 2010). Spherical defects can be formed at high energy density. Figure 4.4 shows typical single slices of a CT scan and locally reconstructed models $(3 \times 3 \times 0.8 \mathrm{~mm})$ of SLM specimens built by Zone II marginal parameters.
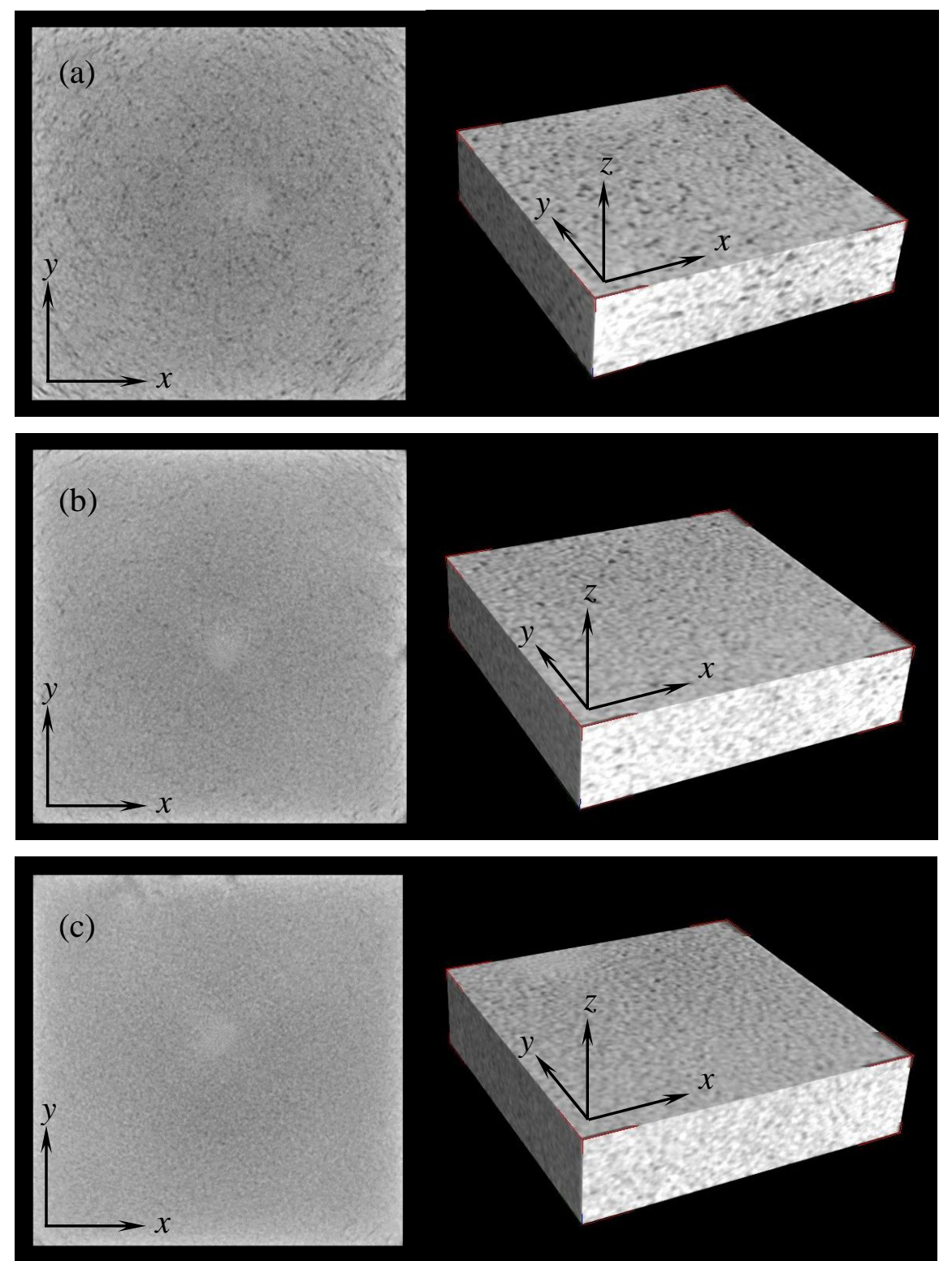

Figure 4.4. Single slices and locally reconstructed models of SLM specimens (Zone II). (a) $V=360 \mathrm{~mm} / \mathrm{s}, R_{D}=6.0 \%$. (b) $V=480 \mathrm{~mm} / \mathrm{s}, R_{D}=2.0 \%$. (c) $V=600 \mathrm{~mm} / \mathrm{s}, R_{D}=0.5 \%$. 
The single slice and reconstructed model present rough surfaces when the SLM specimen has a porosity around 6\%, as shown in Figure 4.4 (a). According to the CT theory, the bulk material is displayed as white while the defect is dark. The single slice shows an irregular texture variation. Thus, it is inferred that there are a large amount of defects within the specimen. However, only a few defects can be observed from the slices and surfaces of the reconstructed model due to the limited CT detectability (dimension $\geq 21 \mu \mathrm{m}$ for visualization), which implies that the dimension of most defects is less, or not significantly larger than $21 \mu \mathrm{m}$ so that they are hardly distinguished from the bulk material. It is notable that the texture of the slice becomes relatively uniform when $R_{D}$ is reduced to $2 \%$, as shown in Figure 4.4 (b). But its defects can still be predicted compared to the grayscale image for the low porosity slice $\left(R_{D}=0.5 \%\right)$, which shows relatively equal distribution of grayscale in Figure 4.4 (c).

Zone II and Zone III of SLM marginal parameters are usually separated by Zone I. Specimens can be fabricated to be fully dense with Zone I processing parameters. But if the laser power is decreased or scan speed is increased beyond Zone I, the processing parameters will be considered as Zone III marginal parameters. The reduced energy density will generate defects with an irregular morphology due to insufficient melting. Figure 4.5 shows micro-CT slices and reconstructed models of SLM specimens fabricated by Zone III marginal parameters. Similar to the slices in Figure 4.4 (c), the slice with low porosity $\left(R_{D}=0.1 \%\right)$ also shows equal grayscale distribution, as shown in Figure 4.5 (a). The increased porosity results in a rough texture in the slices and surface of reconstructed models. It can be inferred that most defects are also randomly distributed within the cross sections of specimens with small dimensions. But it is difficult to identify the defect 
morphology and locate small individual defects within SLM specimens using CT slices and reconstructed models.
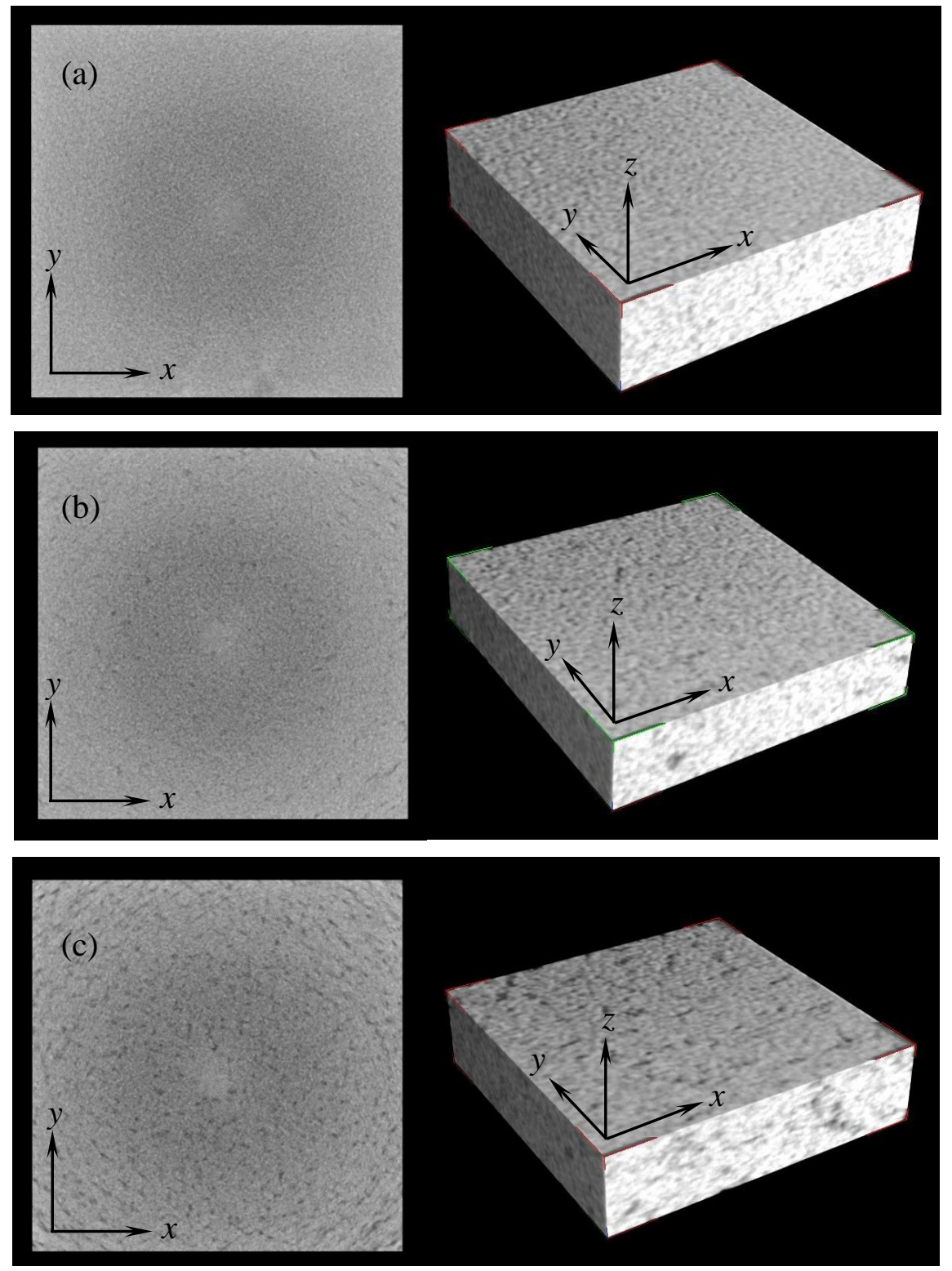

Figure 4.5. Single slices and locally reconstructed models of SLM specimens (Zone III). (a) $V=1200 \mathrm{~mm} / \mathrm{s}, R_{D}=0.1 \%$. (b) $V=1320 \mathrm{~mm} / \mathrm{s}, R_{D}=2.0 \%$. (c) $V=1560 \mathrm{~mm} / \mathrm{s}, R_{D}=6.0 \%$.

\subsubsection{Characteristics of EBM specimens}

Defects of EBM specimen can be detectable directly from the CT slice, even at a low porosity, as shown in Figure 4.6 (a). This indicates that most defects are larger than 
the SLM defects. The grayscale distribution of the non-defective areas is continuous, meaning that the material composition and properties are homogeneous resulting in uniform absorption of the X-ray radiation. A few defects are randomly distributed in a single slice of an EBM specimen.
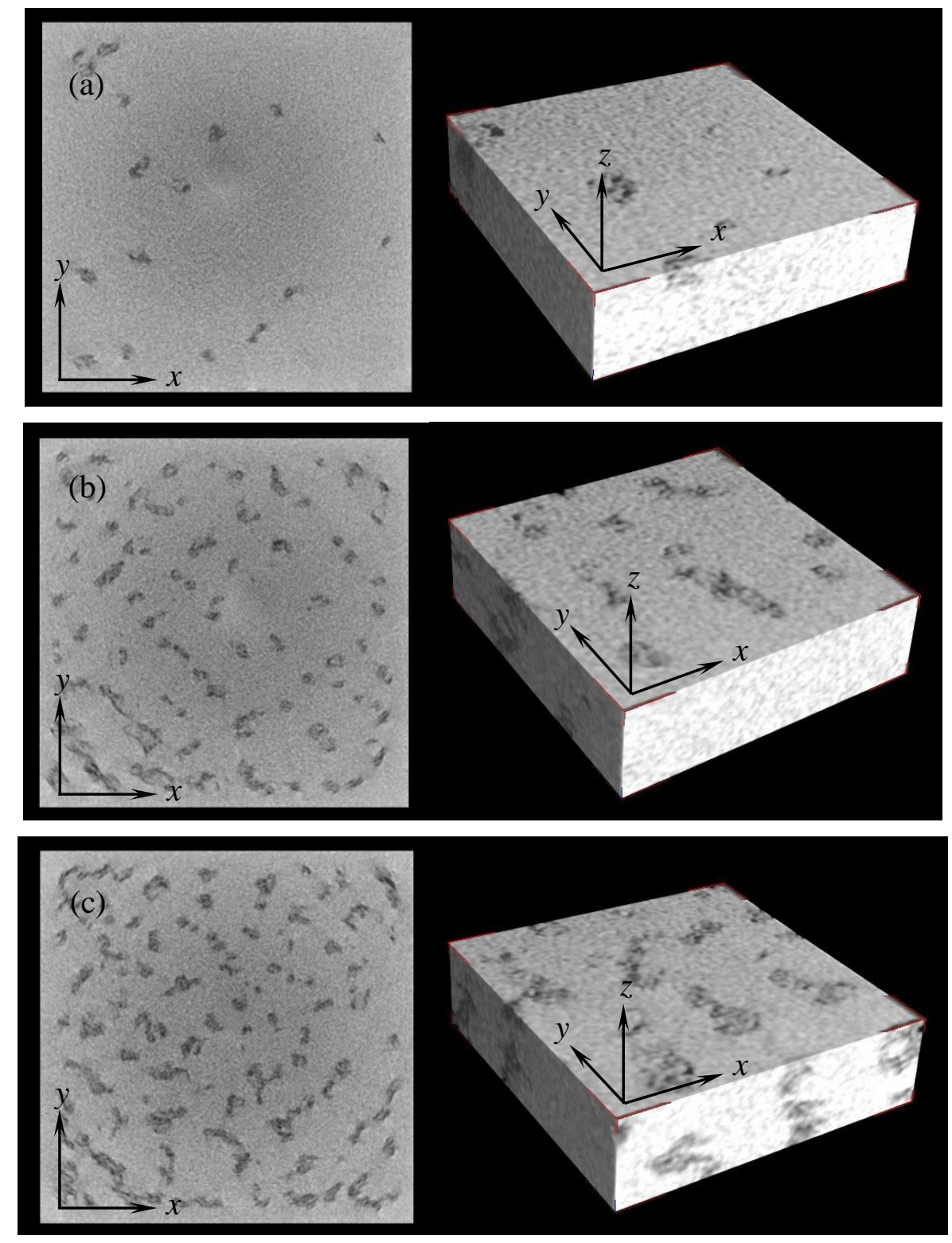

Figure 4.6. Single slices and locally reconstructed models of EBM specimens. (a) $L O=0.18$ $\mathrm{mm}, R_{D}=0.7 \%$. (b) $L O=0.24 \mathrm{~mm}, R_{D}=2.0 \%$. (c) $L O=0.30 \mathrm{~mm}, R_{D}=4.0 \%$. 
Line offset $(L O)$ refers to the distance between two hatch lines, similar to hatch spacing in SLM. The increased line offset will reduce the energy density, which exacerbates defect inclusion within EBM specimens. The defect amount increases with an enlarged line offset value. Higher porosity is usually accompanied with larger defect dimensions. From Figure 4.6 (b) and (c), it can be seen that the stochastic defects are distributed everywhere within the EBM specimens.

Another parameter which affects the generation of defects in EBM specimens is focus offset $(F O) . F O$ is the additional current running through the respective electromagnetic coil and can be translated into an offset of the focal plane from its zero position (Schwerdtfeger et al, 2012). Increasing the focus offset causes an enlarged beam diameter, which lowers the energy density and generates defects. Thus, it is noted that more defects are visible from the CT slices and reconstructed models in Figure 4.7 (b) and (c). The morphological and distributional characteristics of focus offset defects are comparable to that of line offset defects. However, the dimension of focus offset defects is smaller by contrast. This indicates that, for a given porosity, the number of focus offset defects is more than that of line offset defects in EBM specimens.

The scanning results for SLM and EBM specimens show different stochastic defects characteristics for each. Compared to SLM specimens, the defects in an EBM specimen are more easily detected using the micro-CT scanning due to their dimensional features. The morphology and distribution of defects can be well described using single slices or reconstructed models. For scanning of SLM specimens, the dimensions of defects are comparable to or slightly larger than the CT visualization threshold $(21 \mu \mathrm{m})$. The scanning results only show a rough texture even though SLM specimens contain defects at 
a higher porosity level. Thus, SLM slices are not as to use as EBM slices for morphological feature analysis of defects.
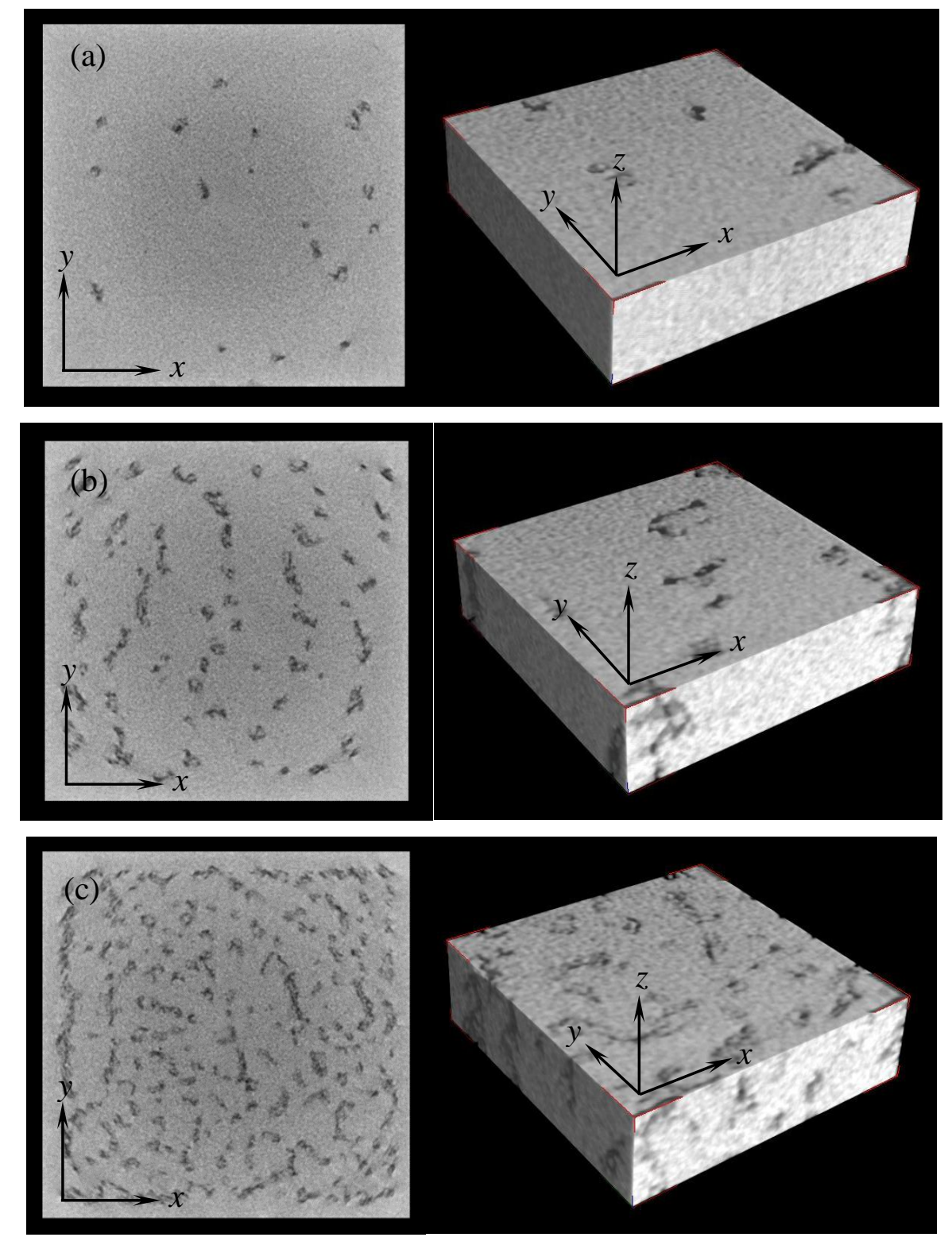

Figure 4.7. Single slices and locally reconstructed models of EBM specimens. (a) $F O=16$ $\mathrm{mA}, R_{D}=0.3 \%$. (b) $F O=20 \mathrm{~mA}, R_{D}=3.0 \%$. (c) $F O=24 \mathrm{~mA}, R_{D}=4.5 \%$.

\subsubsection{Porosity Determination}

\subsubsection{Image Correction to Single Slices}


Due to the prominence of EBM defects, their CT single slices can be utilized for estimating porosity using image processing. Since CT slices intensities are correlated with machine setup and manipulation parameters, the grayscale information can only represent relative attenuation values for describing radiation absorption. Homogeneous material are also expressed by the gradient grayscales in a CT slice of EBM specimen, as shown in Figure 4.8 (a), which illustrates radiodensity inconsistency. Area "A" and "B" are all believed to be alike. But the grayscale of area " $\mathrm{A}$ " (near center) is darker than area B (near boundary). It is hard to select a uniform grayscale threshold to convert CT slices to binary images for highlighting a defective region, and for further porosity calculation. As shown in Figure 4.8 (b), when an intermediate grayscale threshold is applied to the original slice image to convert to a binary image, there are a certain amount of tiny undesired white spots not being filtered (within the dashed circle). However, if a higher grayscale threshold is adopted, the morphological features of defective areas, specifically near a boundary, are altered significantly, although central white spots are removed in Figure 4.8 (c).

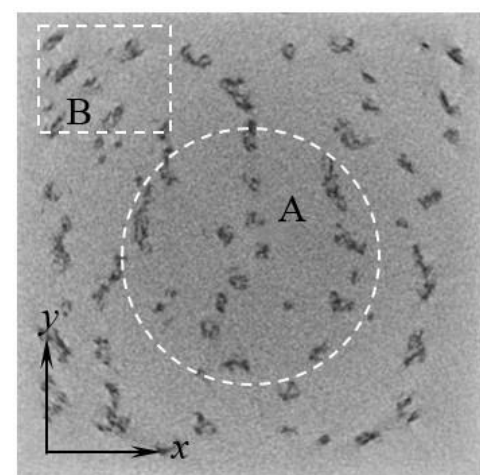

(a) Original slice

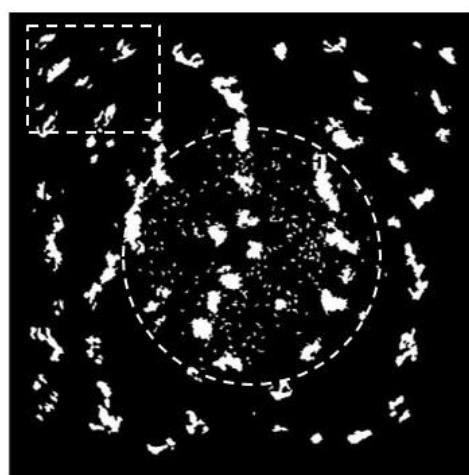

(b) Binary image 1

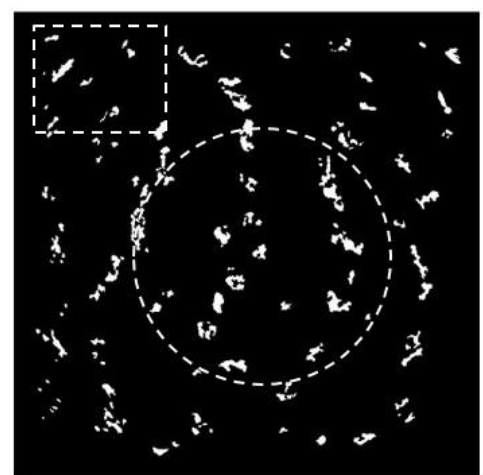

(c) Binary image 2

Fig. 4.8. Image processing of a single slice of a CT scanned EBM specimen.

There are two approaches for solving grayscale inconsistency in a single CT slice image. The first one is to set up a multi-threshold when converting the original image to 
binary. For a darker area, such as central area "A", a higher threshold could be applied, while maintaining a regular threshold in the periphery. However, a boundary between the areas needs to be determined before setting up multi-thresholds. Separate image processing also makes the conversion more complex. An alternative approach is to correct the slice image to equal grayscale levels before converting to a binary image. The corrected image is suitable for further conversion with a uniform threshold. For image correction, the original slice image was first segmented into cells. The maximum grayscale value of each cell was searched and then assigned with an equal value. Correspondingly, the grayscale of other pixels within this cell was proportionally assigned a new value according to the modified maximum grayscale, as shown in Figure 4.9. By doing this, all cells are unified to the same grayscale level.

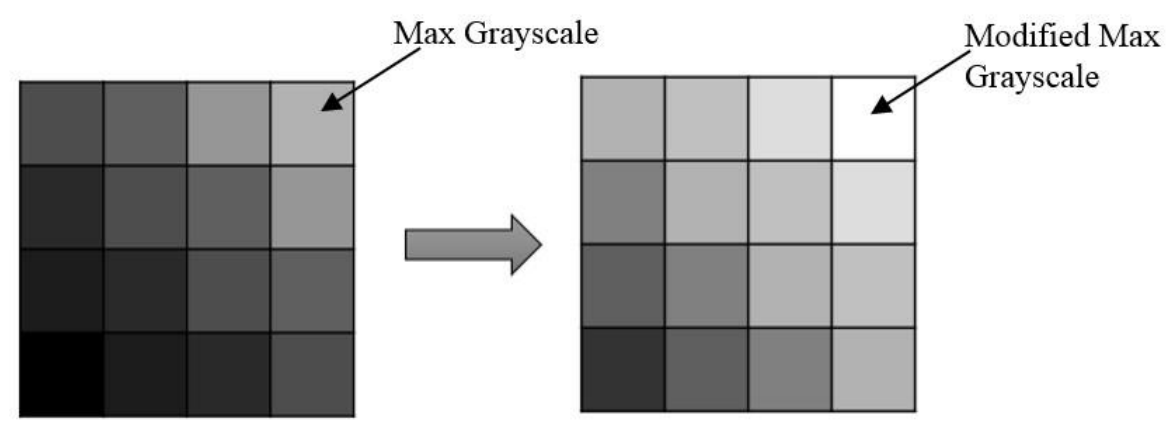

Figure 4.9. Schematic of grayscale adjustment to an individual cell (16 pixels).

An example of image correction is shown in Figure 4.10. It can be seen that the inconsistency of grayscale between the center and boundary is weakened in the corrected image. The morphological features of defects are maintained after correction. The inconvenience of image conversion due to grayscale inconsistency is completely eliminated. The corrected image ensures the accuracy of porosity estimation when being converted to a binary image. 


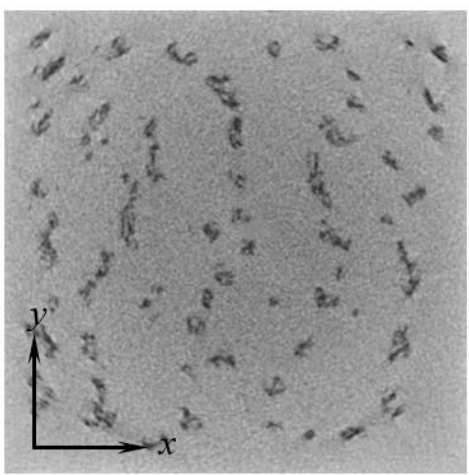

(a) Original slice

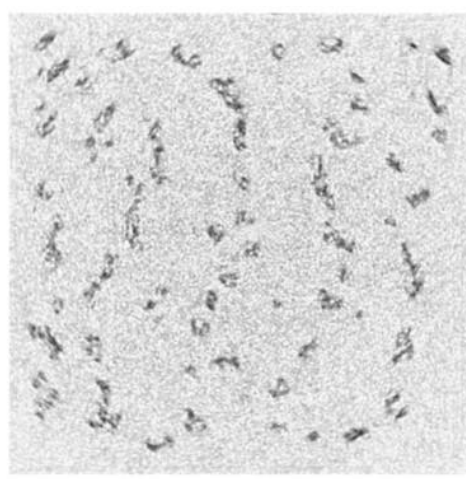

(b) Corrected image

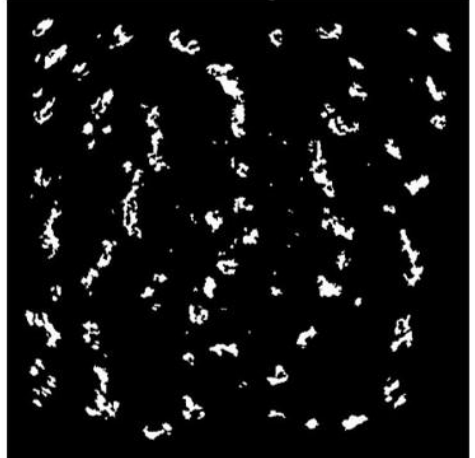

(c) Binary image

Figure 4.10. Example of image correction and binary image.

Through a binary image, defective areas (white color) can be summed and then divided by the entire image area to calculate "image processing porosity $\left(R_{I}\right)$ ". The comparison between $R_{D}$ and $R_{I}$ is shown in Figure 4.11. It can be seen that $R_{I}$ is very close to $R_{D}$ as long as the proper threshold is selected when converting the corrected image to a binary image. Moreover, once a threshold is calibrated for estimating porosity based on one binary image, no further calibration is required for other images, since the corrected images adopt the same grayscale level. Porosity estimation based on single CT slices is feasible for specimens with large internal defects such as porous EBM specimens.

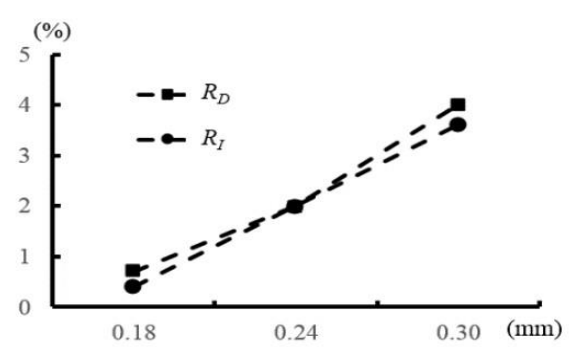

(a) Line offset

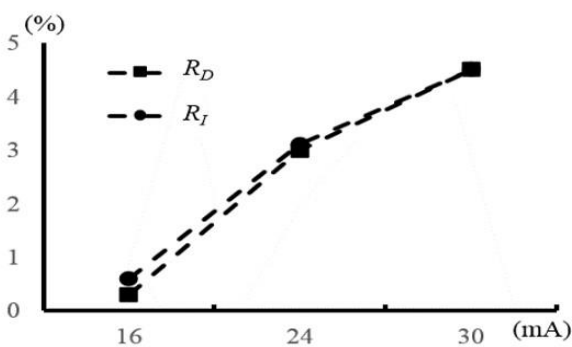

(b) Focus offset

Figure 4.11. Comparison between $R_{D}$ and $R_{I}$.

\subsubsection{Selection of Grayscale Threshold for Reconstructed Model}

Reconstructed models from CT slices consist of a continuous set of voxels, which represents a specific area of the object. The grayscale of each voxel provides information 
about the material properties in this area. In the case of a CT scan, grayscale variation is capable of capturing the characteristics of the reconstructed model, including material properties and inner structures. A well selected grayscale threshold is able to display both voxel and polygon data and to determine the entire structure of object with a high accuracy. In an SLM or EBM CT image, defective regions are distinguished from dense regions by their low grayscale values. Grayscale threshold selection is needed to calibrate porosity in order to match the results of Archimedes measurements. Since absolute grayscale value is change due to the slice brightness or contrast selected during CT setup, it is hard to select a uniform grayscale threshold for all SLM or EBM specimens. Even for a consistent configuration, the grayscale threshold for each specimen is slightly different. Generally, the Hounsfield Unit (HU) is utilized to linearly transform the original CT number (grayscale) of a voxel into a quantitative scale for describing radiodensity (Brown et al, 2008), in order to reduce the effect of CT setup variation. But the radiodensity variation also results in grayscale inconsistency in a reconstructed model. Based on this, it is inferred that $\mathrm{HU}$ transformation does not ensure a uniform grayscale threshold for all scans of SLM or EBM specimens.

A pragmatic method for selecting a grayscale threshold is proposed to approximately estimate porosity of stochastic defects. The grayscale threshold is firstly adjusted for each scan of an SLM or EBM specimen in order to achieve the porosity $\left(R_{D}\right)$. Then, a neutralized threshold is selected for the reconstructed models for a particular manufacturing process. A porosity $\left(R_{M}\right)$ generated by the neutralized threshold is compared to the Archimedes porosity $\left(R_{D}\right)$, as shown in Figure 4.12. 
Basically, the porosity $\left(R_{M}\right)$ has a similar increasing or decreasing trend compared to the Archimedes porosity $\left(R_{D}\right)$. Only Figure 4.12 (b) shows a complete matching between $R_{M}$ and $R_{D}$. Most values of $R_{M}$ are not able to represent the accurate porosity. Apparently, the neutralized grayscale threshold is not the best solution to reflect the amount of stochastic defects through the reconstructed models. However, the grayscale inconsistencies are a result of the mutual influence of CT setup and the radiodensity variations. It could be complicated to compensate for all of these impacts to calibrate grayscale for the reconstructed models. Thus, neutralized grayscale threshold could be employed for an approximated porosity when the CT slices are reconstructed to a threedimensional model, especially for the scan of SLM specimens whose single slices fail to reflect porosity through image correction.

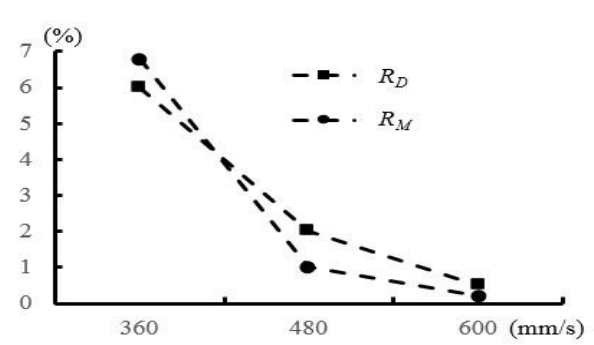

(a) Zone II marginal parameters of SLM

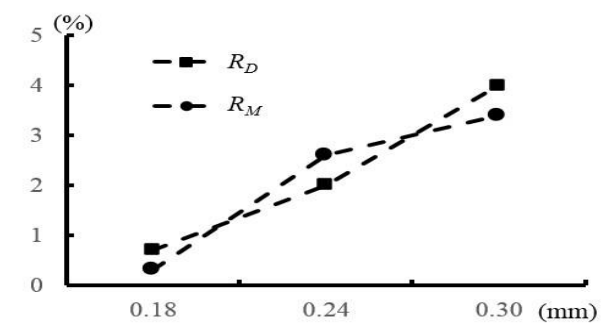

(c) Line offset of EBM

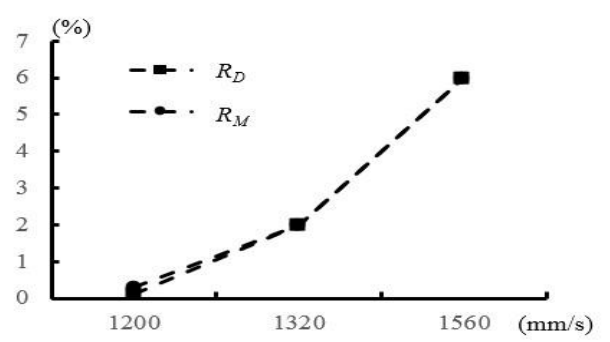

(b) Zone III marginal parameters of SLM

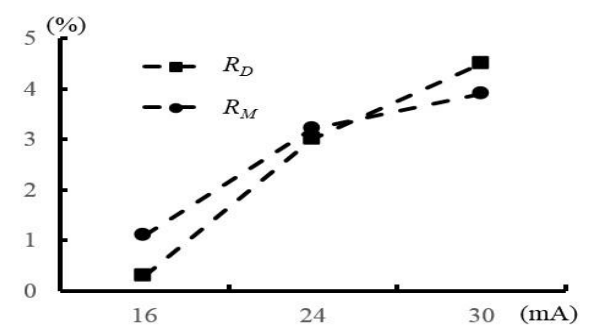

(d) Focus offset of EBM

Figure 4.12. Comparison between $R_{D}$ and $R_{M}$.

\subsection{Conclusion}

Micro-CT is capable of detecting stochastic defects and demonstrating their morphology in the reconstructed models. However, machine setup and CT detectability 
play an important role in defect visualization. Stochastic defects are dissimilar between SLM and EBM processes. In this study, the contour features of SLM stochastic defects are hard to see in the CT scans due to their limited dimensions. But EBM stochastic defects are large enough to be clearly visualized.

Dense specimens usually display a homogeneous grayscale variation in CT scans. But stochastic defects cause irregular variations of brightness and darkness in localized areas of a single slice. The porosity of EBM specimens can be estimated in single slices through image processing, while single slices of SLM specimens only show a rough texture and cannot be used for porosity analysis. Moreover, when stochastic defects are reconstructed into a 3D model, approximated porosity can be obtained by carefully selecting a neutralized grayscale threshold. Although its accuracy is limited compared to the Archimedes measurement, the capability of rapid estimation of porosity is promoted if a proper grayscale threshold is selected. 


\section{CHAPTER 5}

\section{MECHANICAL PROPERTIES OF SLM- AND EBM-PRODUCED TI-6AL-4V WITH DEFECTS 5}

\subsection{Introduction}

Additive Manufacturing (AM) is capable of producing parts directly from threedimensional CAD data, layer upon layer. The geometric characteristics of AM parts can be complicated. Mechanical properties of AM parts are different from conventionally manufactured parts.

Selective Laser Melting (SLM) and Electron Beam Melting (EBM) are two typical powder bed fusion processes within AM (Gibson et al, 2009). The SLM process selectively melts metallic powder with a focused laser beam. The melted powder fuses and solidifies to the previous layer or support structure, while undergoing rapid cooling. After that, a layer of new powder is recoated upon the current layer for subsequent layer melting (Thijs et al, 2010). Many metal powders are utilized for SLM, including stainless steel, maraging steel, cobalt chromium and titanium alloys.

The EBM process uses a focused electron beam to melt powder layers in AM. When the electrons penetrate the powder surface and further into the grains, their kinetic energy

\footnotetext{
${ }^{5}$ This chapter is a paper prepared for Rapid Prototyping Journal. All permissions to use this paper as a part of this dissertation are contained in Appendix D.
} 
is converted into thermal energy, which raises the material temperature above the melting temperature, (Zäh et al, 2010). Titanium and cobalt chromium powder are well developed for EBM by Arcam AB of Sweden.

Due to incomplete wetting and balling effects, defects (such as pores or voids) are easily formed during the SLM process. The formed ball becomes isolated from the rest of the powder by comparatively large pores (Thijs et al, 2010; Tolochko et al, 2004). For low energy input, successive scan tracks do not fuse and large pores appear along the scan lines (Vandenbroucke et al, 2007; Li et al, 2010). Two kinds of pores are found in EBM parts. Bigger pores are elongated in the layer direction. These pores are attributed to the use of non-optimized process parameters in EBM. Smaller spherical pores are due to defects already present in the powder particles caused by gaseous argon entrapped during the atomization process (Biamino et al, 2011).

The mechanical properties of SLM or EBM parts are negatively influenced by porosity. A defect is likely to be a source of preferential crack nucleation and propagation which will reduce the fatigue life in comparison to fully dense material (Sercombe et al, 2011). Santos et al (2002) conducted laser melting of pure titanium powder and fabricated specimens with densities of higher than 95\%. Although the tensile strength tests showed results comparable to wrought material, the impact and torsional fatigue strengths were low because of porosity. Li et al (2012) carried out compression fatigue behavior tests on Ti-6Al-4V mesh arrays with high porosities of $60-85 \%$. It was found that the fatigue strength increased with increasing relative density. Three point bending tests were performed by Kruth et al (2004) to determine the bending strength of SLM iron-based parts. They found that the bending strength increased with material density. Ramirez et al (2011a) 
and Murr et al (2010) studied cellular structures and stochastic foams fabricated by EBM from precursor $\mathrm{Cu}$ and $\mathrm{Ti}-6 \mathrm{Al}-4 \mathrm{~V}$ powder. They proposed that the stiffness or Young's moduli for these cellular structures vary with density and vary inversely with porosity. Vandenbroucke et al (2007) measured the hardness of SLM Ti-6Al-4V samples, and proposed that micro hardness does not vary significantly with porosity. Since macro hardness is more dependent on porosity, the measured value is lower than those for micro hardness.

Defects act as stress concentrators leading to earlier onset of plasticity and localization of strain (Yadroitsev et al, 2009). Thus, porosity is usually detrimental to mechanical strength. However, only a few investigations focused on the effect of stochastic defects on SLM- and EBM-produced parts. Based on the previous experimental results regarding defect generation and distribution, this study aims to correlate the identified porosity to the mechanical properties, such as tensile and fatigue performance, of SLMand EBM-produced Ti-6Al-4V parts. Use of marginal parameters (scan energy different than optimum) generates stochastic defects in SLM- and EBM-produced parts. During the SLM process, optimum parameters (Zone I) are capable of fabricating fully dense SLM parts. Marginal parameters can be subdivided to high energy parameters (Zone II) and low energy parameters (Zone III). Zone II marginal parameters cause spherical defects, while Zone III marginal parameters generate defects with irregular shapes. EBM marginal parameters are similar to Zone III marginal parameters in SLM. Defects result from insufficient energy density compared to the optimum parameters. This study utilizes tensile and fatigue tests to determine the effect of stochastic defects on the properties of SLM- and EBM-produced Ti-6Al-4V specimens in comparison fully dense specimens. 
Microstructural and fractography studies illustrate the cracking mechanisms which resulted from defects.

\subsection{Materials and Methods}

Raymor Ti-6Al-4V powder (Grade 23) with an apparent density of $2.6 \mathrm{~g} / \mathrm{cm}^{3}$ was used for SLM process, with a particle size distribution between $17 \mu \mathrm{m}\left(\mathrm{D}_{10}\right)$ and $44 \mu \mathrm{m}$ (D90) and Mean Volume Diameter around $30 \mu \mathrm{m}$. Many particles are included in Raymor Ti-6Al-4V powder. Arcam Titanium Ti-6Al-4V powder (Grade 23) was used for EBM. Its apparent density is less than $2.7 \mathrm{~g} / \mathrm{cm}^{3}$. The particle size of Arcam Ti-6Al-4V powder is normally distributed between $47 \mu \mathrm{m}\left(\mathrm{D}_{10}\right)$ and $99 \mu \mathrm{m}\left(\mathrm{D}_{90}\right)$ with Mean Volume Diameter around $73 \mu \mathrm{m}$. Both Raymor and Arcam powder particles are spherical.

Cylindrical bars were built in the $Z$ orientation (ISO/ASTM 52921, 2013) in an EOS M270 DMLS system and an Arcam S400 EBM system, respectively. Optimized parameters (OP) and marginal parameters (MP) of SLM and EBM are summarized in Table 5.1 and 5.2. Other parameters were set to a constant value. There are five types of SLM specimens and three types of EBM specimens built for tensile and fatigue testing. No heat treatment was performed after the specimens were finished and removed from build plate.

Table 5.1. Experimental Parameters for SLM Test Specimens (Laser Power 120W, Hatch Spacing 0.1mm, Layer Thickness 0.03mm)

\begin{tabular}{c|c|c|c}
\hline Parameter no. & $\begin{array}{c}\text { Scan speed } \\
(\mathrm{mm} / \mathrm{s})\end{array}$ & $\begin{array}{c}\text { Energy density } \\
\left(\mathrm{J} / \mathrm{mm}^{3}\right)\end{array}$ & $\begin{array}{c}\text { Anticipated } \\
\text { porosity } R_{D}\end{array}$ \\
\hline SLM-OP 1 & 960 & 42 & Zone I $\sim 0 \%$ \\
\hline SLM-MP 2 & 400 & 100 & Zone II $\sim 1 \%$ \\
\hline SLM-MP 3 & 540 & 74 & Zone II $\sim 5 \%$ \\
\hline SLM-MP 4 & 1260 & 32 & Zone III $\sim 1 \%$ \\
\hline SLM-MP 5 & 1500 & 27 & Zone III $\sim 5 \%$ \\
\hline
\end{tabular}


Table 5.2. Experimental Parameters for EBM Test Specimens (Layer Thickness 0.05mm)

\begin{tabular}{c|c|c|c|c|c}
\hline Parameter no. & $\begin{array}{c}\text { Max } \\
\text { Current } \\
(\mathrm{mA})\end{array}$ & $\begin{array}{c}\text { Speed } \\
\text { Function } \\
\text { Index }\end{array}$ & $\begin{array}{c}\text { Line Offset } \\
(\mathrm{mm})\end{array}$ & $\begin{array}{c}\text { Focus } \\
\text { Offset (mA) }\end{array}$ & $\begin{array}{c}\text { Anticipated } \\
\text { porosity } R_{D}\end{array}$ \\
\hline EBM-OP 1 & 21 & 98 & 0.1 & 3 & $0 \%$ \\
\hline EBM-MP 2 & 30 & 60 & 0.2 & 15 & $\sim 1 \%$ \\
\hline EBM-MP 3 & 20 & 180 & 0.2 & 5 & $\sim 5 \%$ \\
\hline
\end{tabular}

Metallographic specimens were prepared using a standard grinding and polishing procedure, and etched by Kroll's reagent. An Olympus MX51 Optical Microscope (OM) was used for microstructural characterization. Cylindrical bars were then machined to a circular cross-section conforming to ASTM E8 for tensile testing and ASTM E466 for fatigue testing. Tensile tests were carried out using an Instron 5569A tensile testing machine with Bluehill ${ }^{\circledR} 2$ Testing Software. Tensile specimens were subjected to a controlled tension until failure (ramp rate $2.5 \mathrm{~mm} / \mathrm{min}$ ). High cycle fatigue tests were performed on a $10 \mathrm{kN}$ Instron Electropulse 10000 fatigue testing machine with WaveMatrix $^{\text {TM }}$ Testing Software. Sinusoidal load was applied to fatigue specimens axially at a frequency of $50 \mathrm{~Hz}$ and stress ratio of $R=0.1$. Fatigue tests were stopped when the specimens broke or the fatigue cycles reached $10^{7}$ cycles. All tests were conducted at room temperature. After the tests were completed, the fracture surface was examined using a FEI Nova NanoSEM 600 Scanning Electron Microscope (SEM) for identifying the crack characteristics of both tensile and fatigue specimens.

5.3 Results and Discussion

5.3.1 Microstructure 
SLM-produced specimens undergo a rapid cooling rate which results in a lenticular martensitic ( $\alpha^{\prime}$ phase, hcp) microstructure, as shown in Figure 5.1 (a) and (b). For EBM, the temperature of the build chamber is maintained between 650 and $700{ }^{\circ} \mathrm{C}$. Since EBMproduced Ti-6Al-4V specimens cool down to the room temperature at a slow cooling rate, their microstructure is mainly composed of an $\alpha$ phase (hcp) and a small amount of $\beta$ phase (bcc) within the prior $\beta$ columnar grains. The $\alpha$ phase possesses a lamellar morphology with $\beta$ surrounding the $\alpha$ lamellae boundary, as shown in Figure 5.1 (c) and (d). However, it is found that defect generation has little effect on the phase structure for SLM or EBM specimens. Microstructural evolution primarily depends on cooling rate.
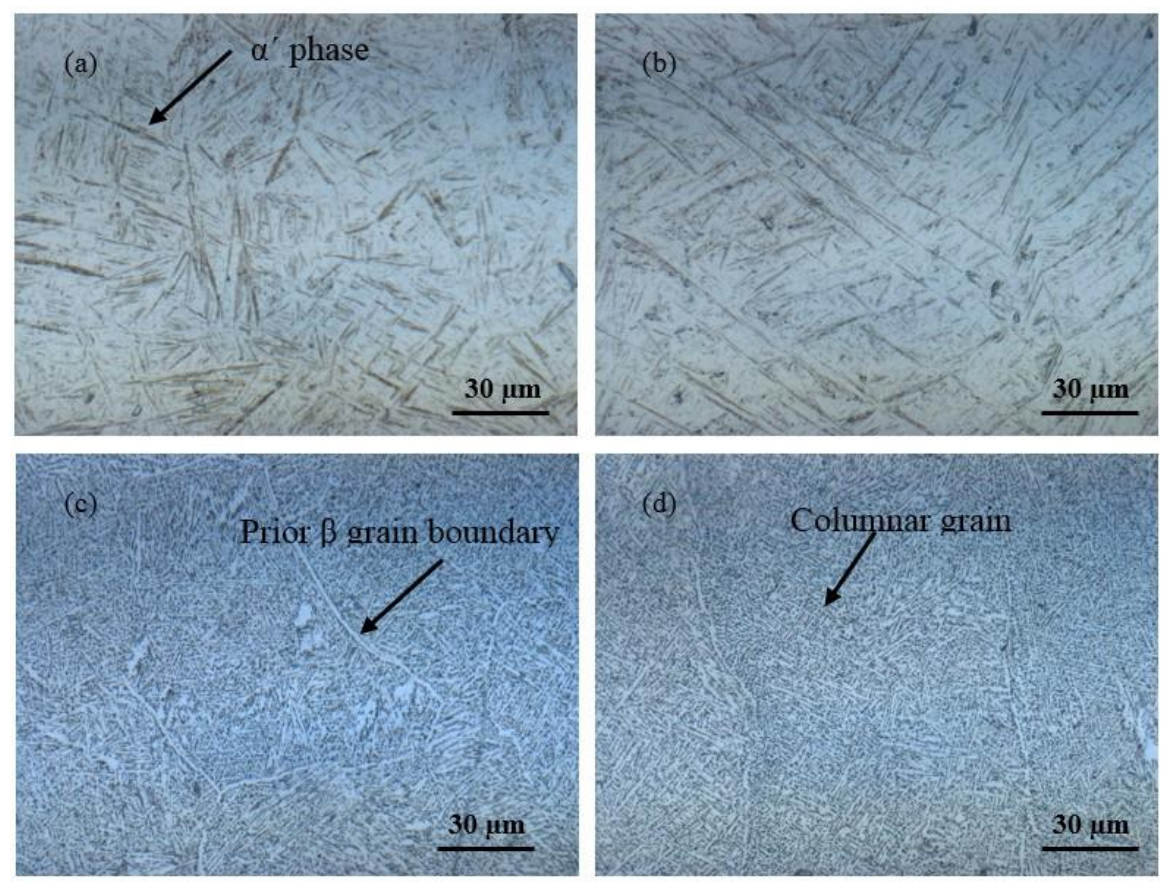

Figure 5.1. Optical microstructure. (a) Horizontal cross-section of SLM specimen; (b) vertical cross-section of SLM specimen; (c) horizontal cross-section of EBM specimen; (d) vertical cross-section of EBM specimen.

\subsubsection{Tensile Properties}

Six duplicate tests were performed for each type of specimen. A static axial clip-on extensometer was attached to the gage section of testing specimens in order to relate the 
stress and strain. Corresponding tensile stress-strain curves were obtained directly from the Instron tensile testing system, as shown in Figure 5.2.

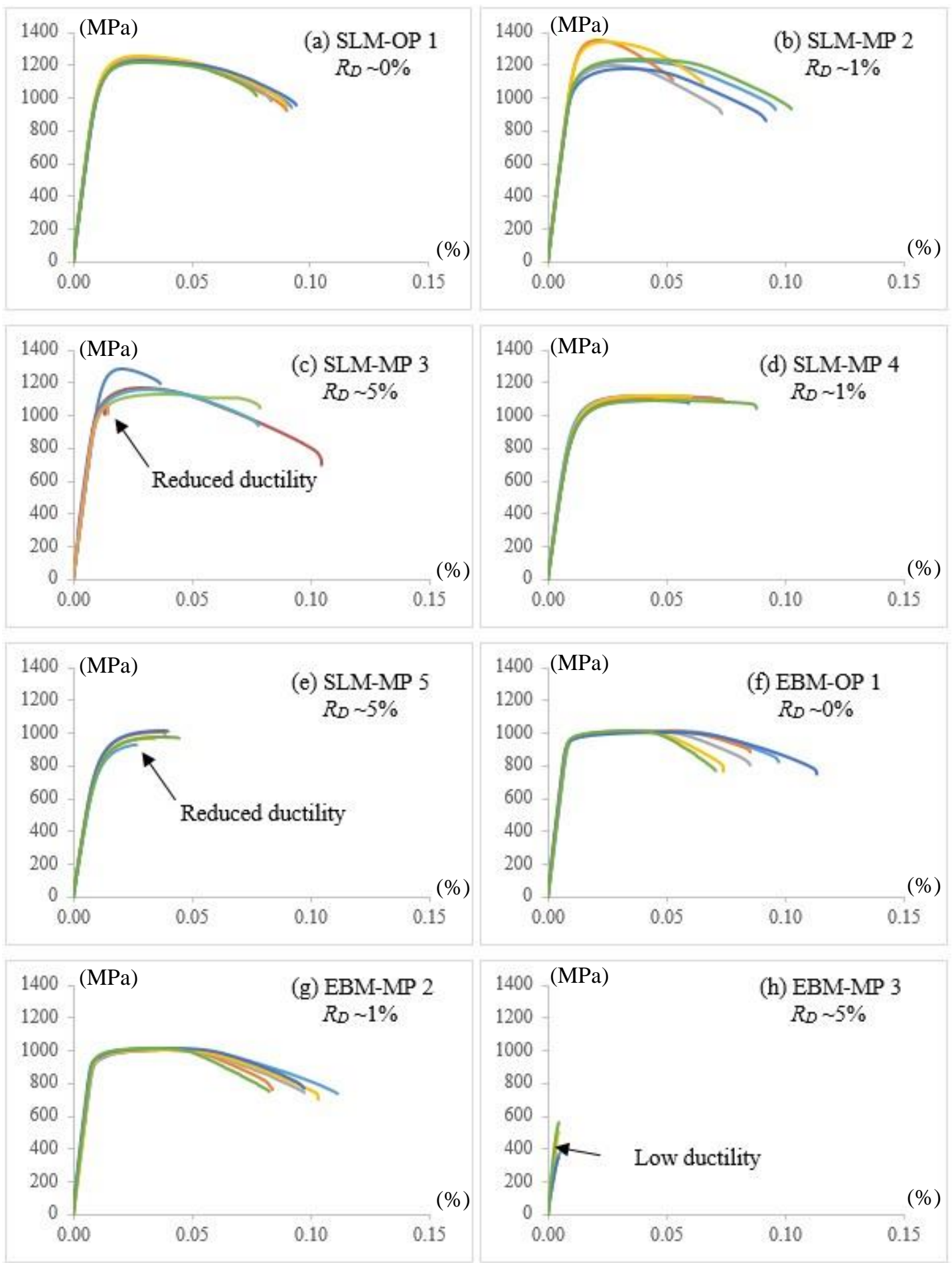

Figure 5.2. Stress-strain plots of SLM- and EBM-produced Ti-6Al-4V specimens. 
Tensile properties, such as yield strength, ultimate tensile strength (UTS), strain at break, and elastic modulus, are summarized in Table 5.3.

Table 5.3 Tensile Properties of SLM- and EBM-Produced Ti-6Al-4V Specimens

\begin{tabular}{c|c|c|c|c}
\hline $\begin{array}{c}\text { Processing } \\
\text { parameter }\end{array}$ & $\begin{array}{c}\text { Stress at yield } \\
{[\text { Offset 0.2\%] }(\mathrm{MPa})}\end{array}$ & $\begin{array}{c}\text { UTS } \\
(\mathrm{MPa})\end{array}$ & $\begin{array}{c}\text { Strain at } \\
\text { break }(\%)\end{array}$ & $\begin{array}{c}\text { E-modulus } \\
(\mathrm{GPa})\end{array}$ \\
\hline SLM-OP 1 & $1098\left(15^{*}\right)$ & $1237(13)$ & $8.8(0.6)$ & $109(2.1)$ \\
\hline SLM-MP 2 & $1150(91)$ & $1257(74)$ & $8.0(2.0)$ & $111(1.4)$ \\
\hline SLM-MP 3 & $1066(91)$ & $1148(80)$ & $5.4(3.8)$ & $109(3.7)$ \\
\hline SLM-MP 4 & $932(16)$ & $1112(13)$ & $6.6(1.4)$ & $95(3.0)$ \\
\hline SLM-MP 5 & $813(23)$ & $978(32)$ & $3.7(0.6)$ & $84(3.0)$ \\
\hline EBM-OP 1 & $962(4.0)$ & $1012(3.0)$ & $8.8(1.6)$ & $121(3.0)$ \\
\hline EBM-MP 2 & $947(11)$ & $1011(4.0)$ & $9.0(1.1)$ & $120(9.0)$ \\
\hline EBM-MP 3 & N/A & $423(88)$ & $0.4(0.1)$ & $92(20)$ \\
\hline
\end{tabular}

* Standard Deviation

When built using optimized parameters (SLM-OP 1 or EBM-OP 1), SLM and EBM specimens possess repeatable tensile strength compared to that of a previous study where SLM-produced Ti-6Al-4V specimens have higher tensile strength than EBM-produced specimens (Rafi et al, 2013). Generally, the hexagonal martensite in SLM specimens provide a higher yield strength and UTS than the $\alpha+\beta$ microstructure of EBM specimens. On the other hand, EBM specimens present comparable or slightly better characteristics of strain and modulus compared to SLM specimens. Interestingly, SLM and EBM specimens built by marginal parameters SLM-MP 2, SLM-MP 3 and EBM-MP 2 show similar tensile properties to optimally produced specimens even though defects are present. "Necking" phenomenon is also observed within the fracture zone within the gage section. Representative topographical features of the fracture surface are shown in Figure 5.3.

In Figure 5.3 (a), the SLM fracture surface displays a mixed mode of brittle fracture and ductile fracture. Brittle fracture is primarily characterized by transgranular cleavage 
facets along low index crystallographic planes (Dieter, 1986). Some localized cup-andcone structures were possibly due to a few defect inclusions which initiate cracking along the plane of maximum shear stress.

EBM tensile specimens also exhibit a cup-and-cone feature in a macroscopic manner. But there are no localized cup-and-cone structures on the fracture surface of EBM specimens. Their fracture surface is mainly characterized by ductile dimples, which are formed by enlarged and coalesced microvoids, as shown in Figure 5.3 (b).
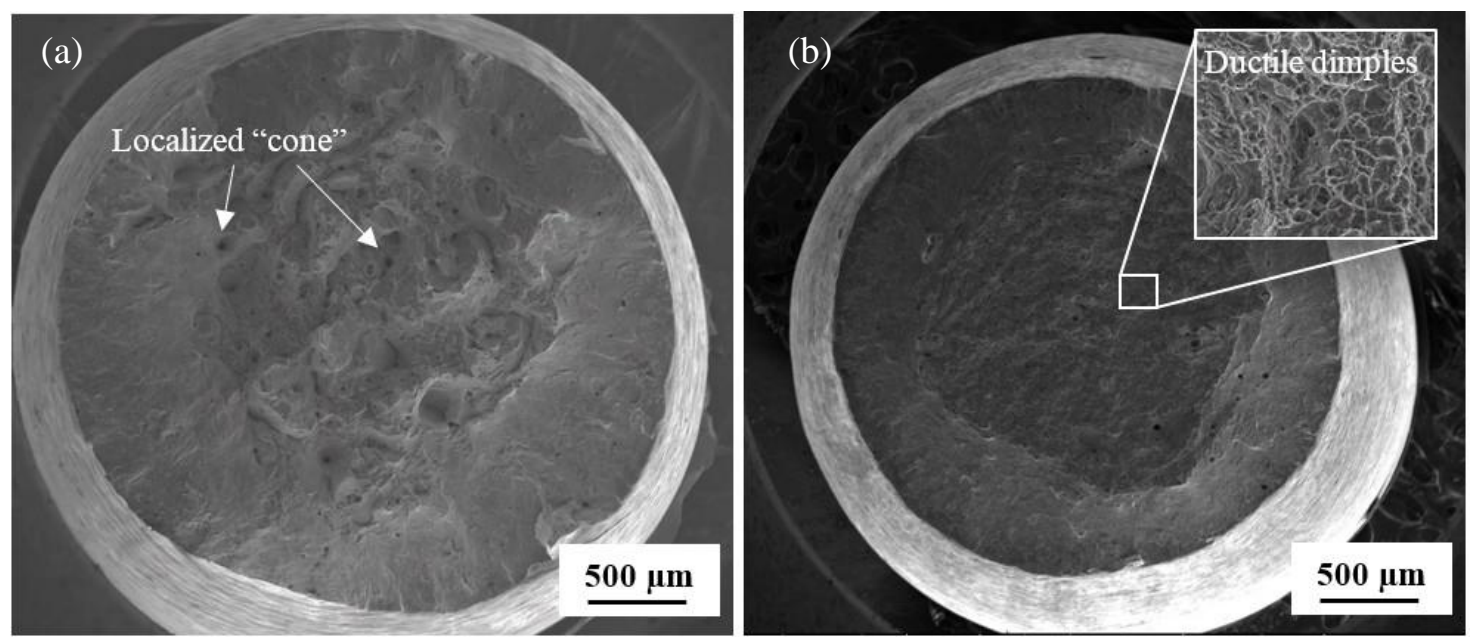

Figure 5.3. SEM fractographs of tensile specimens. (a) SLM (porosity 1\%, Zone II); (b) EBM (porosity 1\%).

An increase in defect density in specimens cause the tensile properties to degrade especially the ductility. For example, the SLM specimens built by SLM-MP 3 parameters at a high energy deposition contain about $5 \%$ of defects $\left(R_{D} \sim 5 \%\right)$. The standard deviation of strain value indicates occasionally reduced ductility of SLM specimens. Some specimens broke immediately after initial yielding. In Figure 5.4, the fractograph shows a rough topographical characterization with reduced ductility. It can be seen that many spherical defects are included and concentrated in the SLM specimens. These defects are the crack initiation sites which result in rapid crack propagation. Thus, no necking occurred. 

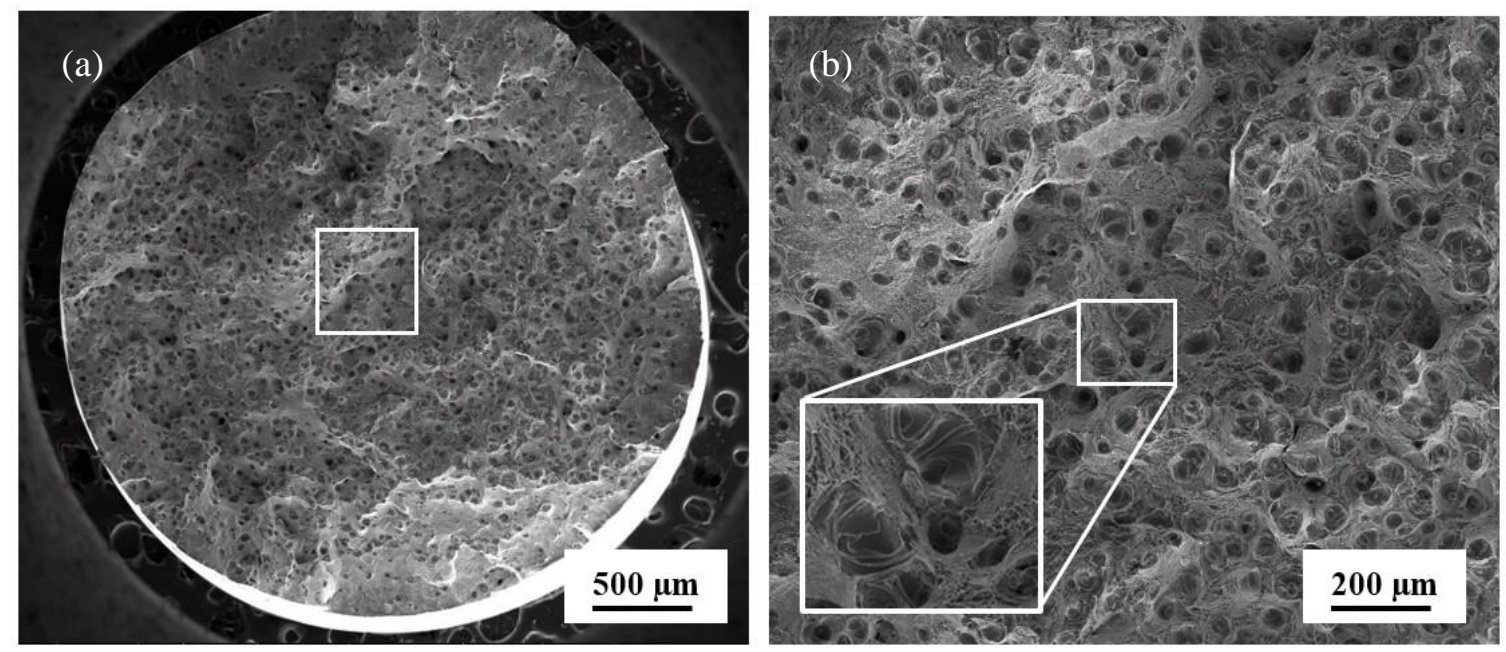

Figure 5.4. SEM fractographs of tensile specimens (a) SLM (porosity 5\%, Zone II); (b) enlarged view from the boxed region in '(a)'.

Specimens built using marginal parameters such as SLM-MP 4, SLM-MP 5, or EBM-MP 3 contain a large number of defects due to incomplete melting. Insufficient energy deposition deteriorates the overall tensile properties which result in rapid transformation from elastic to plastic deformation under a uniaxial load, and then rupture. SLM and EBM tensile specimens were easily broken in the strain hardening phase, even in the typical elastic region. Figure 5.5 (a) and (b) show a fracture surface of an SLM specimen built using SLM-MP 4. The fracture primarily resulted from ductile tearing. The partial melted sites are distributed across the fracture surface. This situation is even worse for SLM-MP 5 and EBM-MP 3 specimens, as powder was sintered and entrapped in the defects. Figure 5.5 (c) and (d) show a fracture surface of an EBM specimen with limited ductile fracture sites, corresponding to a low ductility (see Figure $5.2(\mathrm{~h})$ ).

Based on tensile fractography, tensile properties primarily depend on the microstructure of SLM and EBM specimens when defect inclusion is at a lower level (such as $R_{D} \sim 1 \%$ ). By contrast, increased defect inclusion (such as $R_{D} \sim 5 \%$ ) causes defects to have a greater negative impact on the tensile strength than microstructure. 

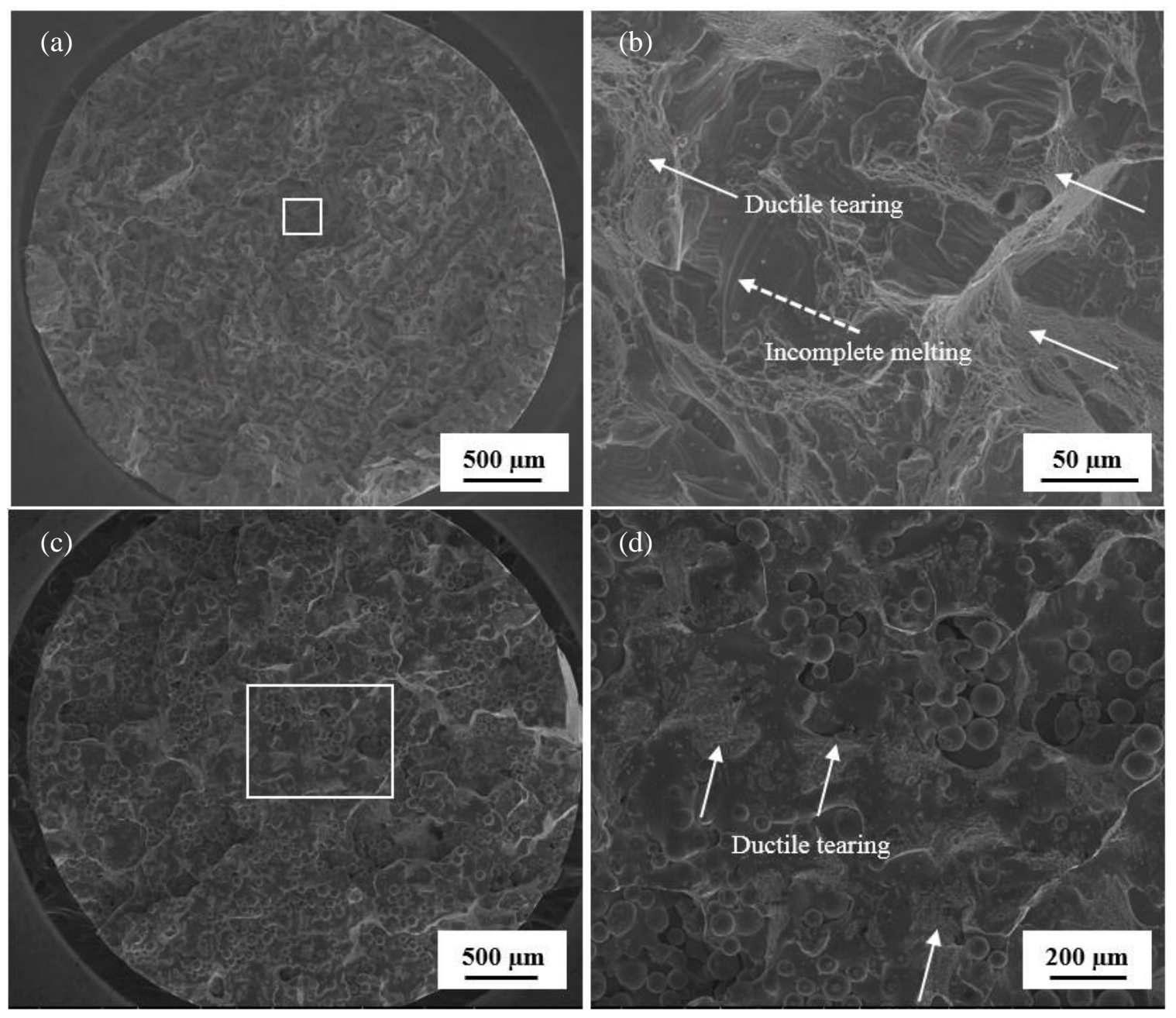

Figure 5.5. SEM fractographs of the tensile specimens. (a) SLM (porosity 1\%, Zone III); (b) enlarged view from the boxed region in '(a)'; (c) EBM (porosity 5\%); (d) enlarged view from the boxed region in '(c)'.

\subsubsection{Fatigue Properties}

Generally, fatigue fracture occurs when cyclic loading is applied above a threshold. Microscopic cracks will begin to form at the stress concentrators such as the surface, persistent slip bands (PSBs), and grain interfaces (Kim et al, 1978). The application of marginal parameters provides a good opportunity for characterizing fatigue performance when stochastic defects are placed in advance, and identifying cracking mechanism of asbuilt SLM and EBM fatigue specimens. Under a high-cycle situation, fatigue performance 
is illustrated by the magnitude of a cyclic stress $(S)$ against the logarithmic scale of cycles to failure $(N)$.

The fatigue data of SLM specimens (SLM-OP 1, MP 2 and MP 3) present a scattered distribution, as shown in Figure 5.6. It indicates that the specimens show a very different failure cycles, even two orders of magnitude more than one other at a specific cyclic load. This could be attributed to the defects inclusion in these specimens. Occasional defects may arise even if a specimen is built using optimized parameters. For the fully dense specimens built by SLM-OP 1, the occasional defect formed in the gage section has a negative effect on the fatigue performance. It may work as a stress concentrator which causes a rapid rupture during the fatigue tests. As for the specimens built by the marginal parameters SLM-MP 2 and MP 3, the included stochastic defects are more liable to cause an irregular fatigue life of SLM specimens. The fatigue limit of SLM specimens (SLMMP $2, R_{D} \sim 1 \%$ ) is lower than $350 \mathrm{MPa}$, which is comparable to that of optimally produced specimens (SLM-OP $1, R_{D} \sim 0 \%$ ). This value is relatively low compared to the fatigue limit (550 MPa) of SLM specimens fabricated using EOS supplied Ti-6Al-4V powder when using the default processing parameters in the EOS machine (Rafi et al, 2013). Both powder and parameter changes may be the cause of the fatigue performance difference between these two types of fully dense SLM specimens. When more defects are included, such as SLM specimens built using SLM-MP $3\left(R_{D} \sim 5 \%\right)$, the fatigue limit is slightly lowered to $300 \mathrm{MPa}$. If parts are built using low energy marginal parameters (such as SLMMP 4 or MP 5), the fatigue specimens display a regular progressed plot with decreased cyclic stress. The fatigue limits are all around $100 \mathrm{MPa}$ at porosity $\sim 1 \%$ and $\sim 5 \%$. It can be inferred that the fatigue performance is seriously deteriorated due to lack of fusion. 


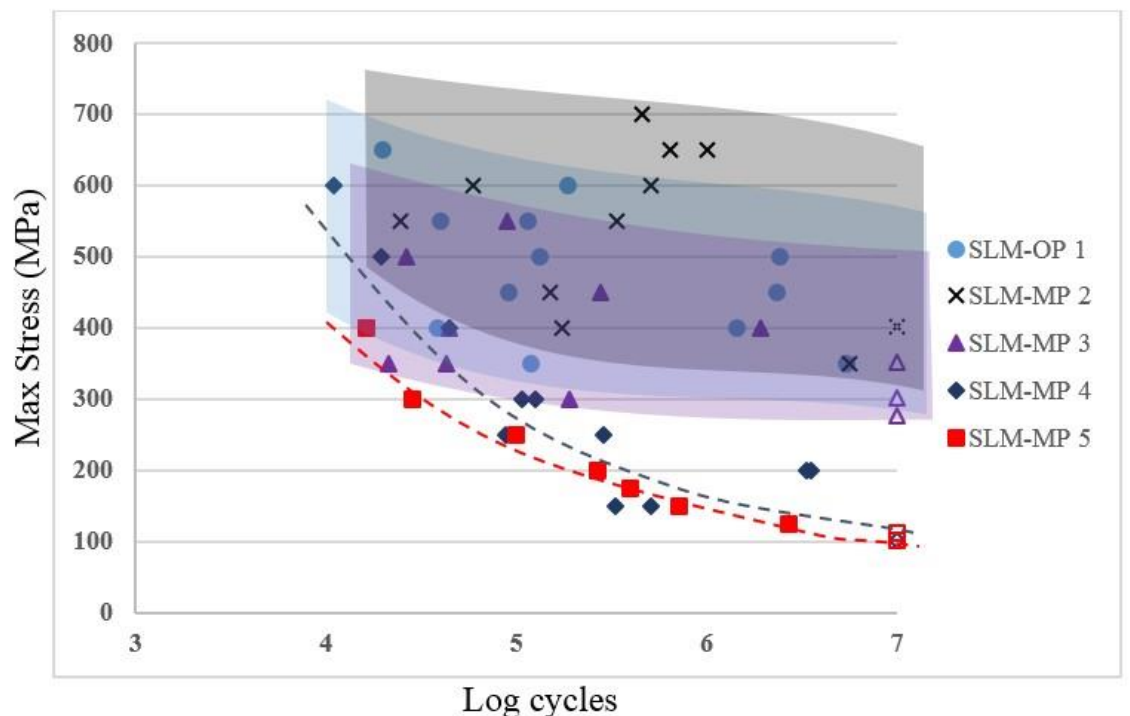

Figure 5.6. Fatigue life of SLM-produced Ti-6Al-4V specimen $(R=0.1$, load controlled smooth gage).

Fatigue fractography shows that the crack always initiates from a defect near the surface or subsurface, and propagates to the whole fracture surface until final failure, even in a SLM specimen which is built using optimized parameters, as shown in Figure 5.7.
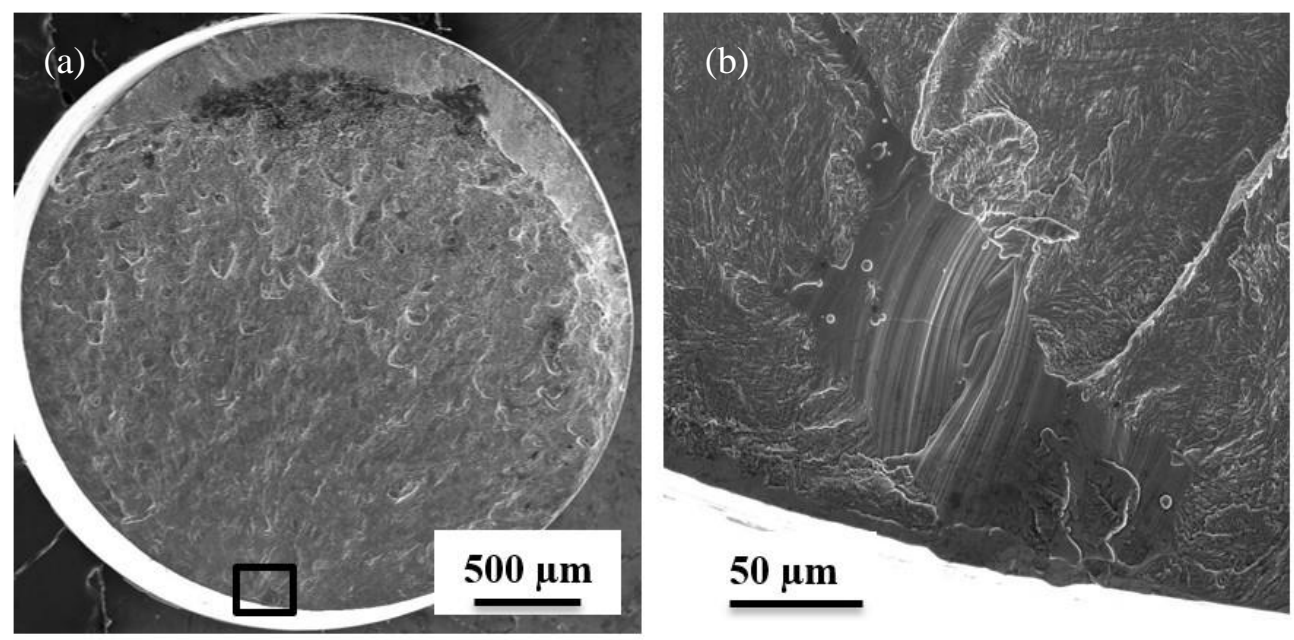

Figure 5.7. Fatigue fractography of SLM-produced fatigue specimens by SLM-OP1. (a)Fracture surface; (b) enlarged view in '(a)'.

This result is different from results seen by Rafi et al (2013) where under certain circumstances Ti-6Al-4V specimens had cracks initiate from interior defects or grain 
interfaces. It could be that if a greater number of specimens of the type used in this study were tested, crack initiation may have also occurred in the interior of the specimens, or it could be that the combination of powder type and process parameters used for this study always results in subsurface defects large enough to be the crack initiation site. Further investigation of this difference may be merited as future work.

Figure 5.8 (a) and (b) show a fracture surface of a fatigue specimen (by SLM-MP 2, $R_{D} \sim 1 \%$ ) containing spherical defects. The defect near the gage surface underwent the largest stress so that the crack was initiated and propagated through multiple crystallographic planes to the interior. Cracking mode was changed to ductile dimples in the overload region which accounts for a minor proportion of the fatigue life. By contrast, defects caused by Zone III marginal parameters (SLM-MP 4 and MP 5) are irregular and show apparent incomplete fusion features, with sintered particles inside. Figure 5.8 (d) shows a typical crack initiation site which initiates transgranular cracks and causes a series of radiating ridges.

Besides the fracture mode, fractography is also able to show three-dimensional morphological characteristics of defects. In Figure 5.4 (b) and 5.8 (b), it can be seen that the spherical defects fabricated by Zone II marginal parameters have a "stair" feature on their internal surface that may merit further investigation. The spherical defects could result from gas bubbles entrapped or generated when a high laser energy is applied to the melt pool ( $\mathrm{Li}, 2006)$, or from the pits generated by the recoater blade when removing welded particles, as discussed in Chapter 2. But according to the morphological features and distribution, gas bubble produced defects likely account for most of Zone II defects in the SLM specimens. 

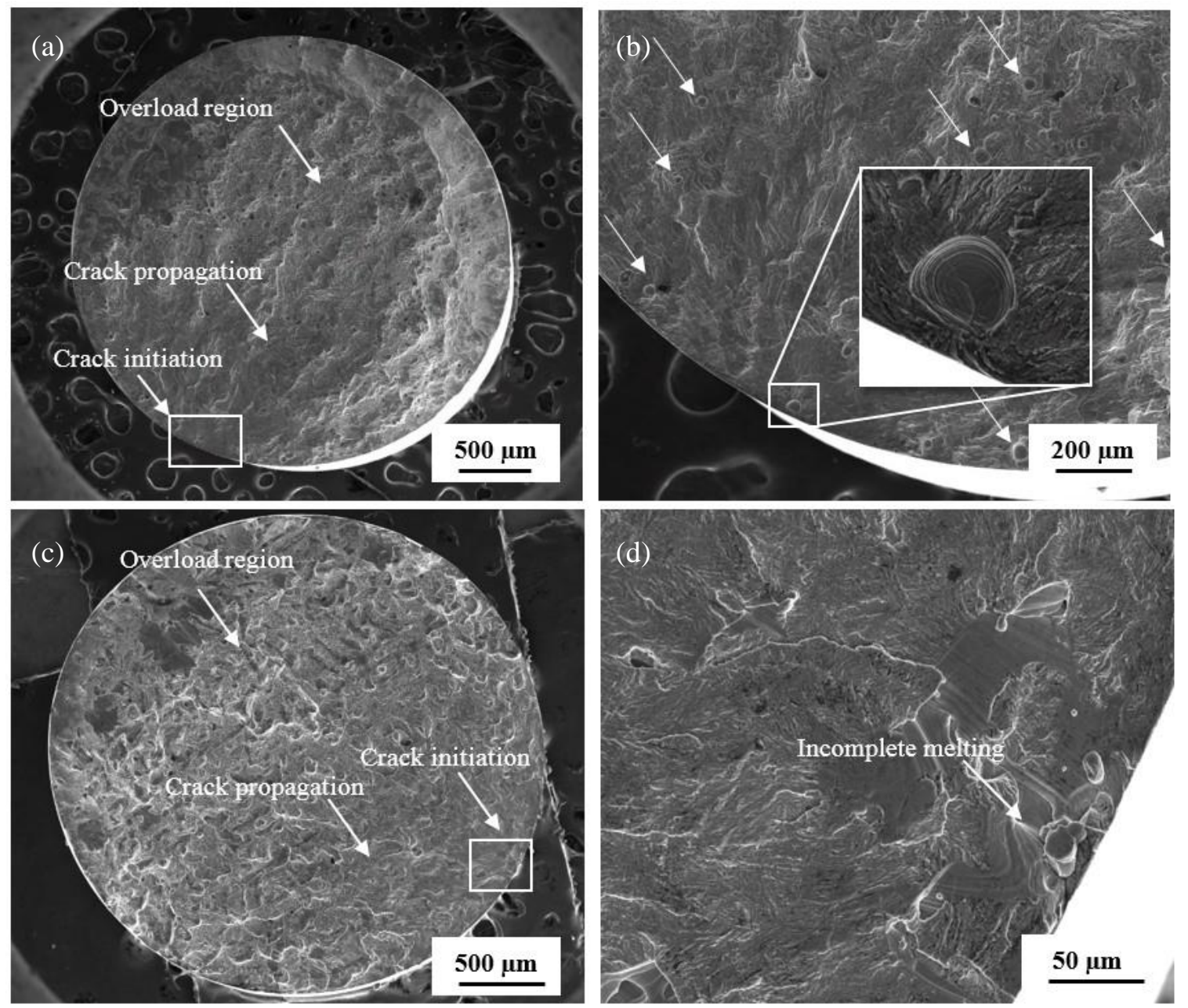

Figure 5.8. Fatigue fractography of SLM-produced fatigue specimens. (a) Fracture surface (porosity 1\%, Zone II); (b) enlarged view in '(a)'; (c) fracture surface (porosity 1\%, Zone III); (d) enlarged view in '(c)'.

As for the Zone III defects, the defect contour is irregular, as shown if Figure 5.5

(b) and 5.8 (d). Localized incomplete melting sites are observable on the fracture surface.

Based on the fatigue stress-cycles curve, it can be deduced that Zone III defects is more detrimental to the fatigue performance of SLM specimens. Martensitic microstructure is not able to weaken initiation and propagation of cracks under cyclic loads, as a result of lack of fusion. If the powder is fully melted by Zone I or Zone II parameters, both defects and martensitic microstructure have a critical impact on SLM specimens. Martensite of 
SLM-produced Ti-6Al-4V impedes dislocation motion and thus leads to a strengthening effect (Rafi et al, 2013). But the appearance of defects may result in serious decrease of fatigue life. Thus, the fatigue date points show a scattered distribution in the stress-cycles curve.

The optimally produced EBM specimens (by EBM-OP 1) show a higher fatigue limit (450 MPa) compared to the SLM specimens. Fatigue data are also distributed in a scattered pattern, as shown in Figure 5.9.

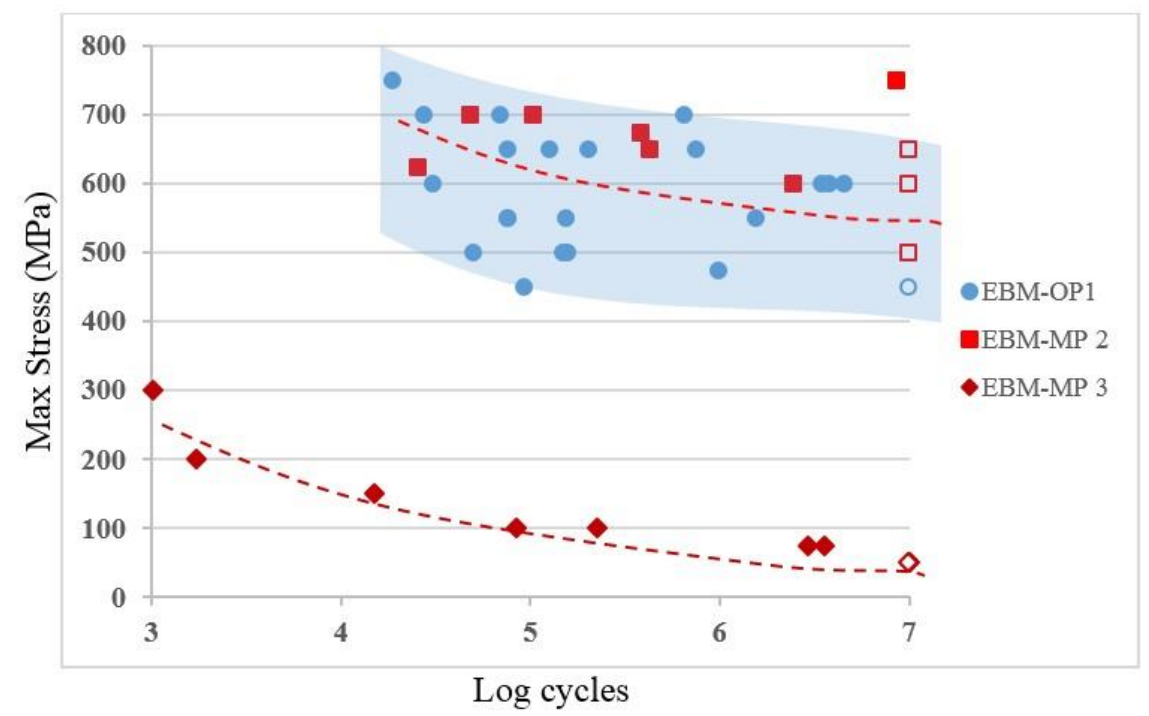

Figure 5.9. Fatigue life of EBM-produced Ti-6Al-4V specimens $(R=0.1$, load controlled smooth gage).

Specimens built by marginal parameters (EBM-MP $\left.2, R_{D} \sim 1 \%\right)$ have a comparable fatigue performance to optimally fabricated parts using EBM-OP 1. There is no statistical difference even though a small amount of defects are included. The superior fatigue strength of EBM specimens indicates that the lamellar $\alpha$-phase has better resistance to cyclic loading, compared to SLM specimens. However, when the porosity is increased to $\sim 5 \%$, the fatigue limit is sharply decreased to $50 \mathrm{MPa}$. 
Figure 5.10 (a) shows the fracture surface for crack initiation from an internal defect. The stress localization around the defect contour could cause crack initiation along the slip bands, as shown in Figure 5.10 (b). The cracks propagate radially outwards through multiple crystallographic planes. However, when the porosity of an EBM-produced specimen is increased to $\sim 5 \%$, lack of fusion causes a weak connection between layers, as shown in Figure 5.10 (c) and (d). The enlarged view shows that the unconnected layers could be easily torn with ductile dimples on the fracture surface.
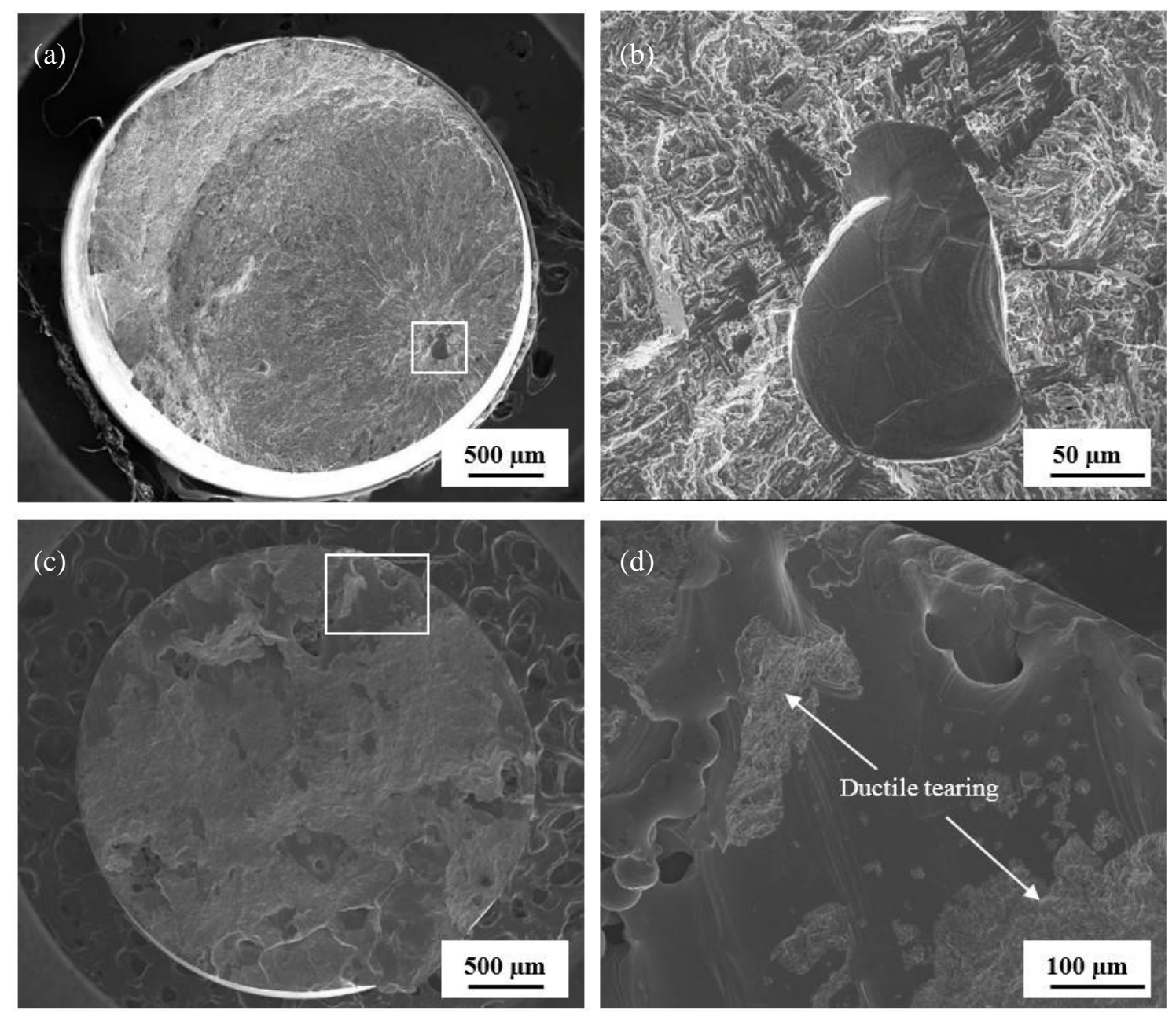

Figure 5.10. Fatigue fractography of EBM-produced fatigue specimens. (a) Fracture surface (porosity 0\%); (b) Enlarged view in '(a)'; (c) fracture surface (porosity 5\%); (d) enlarged view in '(c)'. 
Due to the dual phase microstructure of EBM specimens, cracks are prone to start at the boundaries of the $\alpha$ colonies or of slip bands within the $\alpha$ lamellae according to previous research (Rafi et al, 2013). The fatigue fractography indicates that cracks are always initiated either at the surface or at the subsurface or defect site. It is found that, even when built using marginal parameters, EBM specimens $\left(R_{D} \sim 1 \%\right)$ have similar fracture characteristics compared to optimally produced specimens. Occasional voids are observed on the fracture surface even though optimized parameters are used. This situation is similar to the fatigue performance of fully dense specimens fabricated by SLM-OP1.

As for the fatigue fracture surfaces of SLM and EBM specimens, one common feature is that crack initiation is prone to taking place from defects near the gage surface or subsurface which is highly stressed region of Ti-6Al-4V specimens.

\subsection{Conclusion}

In this chapter, defects were intentionally produced in Ti-6Al-4V specimens using marginal parameters during SLM and EBM processing. The influence of defects on mechanical properties such as tensile and fatigue strength was investigated based on microstructure and porosity.

It was found that the microstructure of SLM and EBM specimens mainly depend on cooling rate. Defect generation does not influence the microstructural evolution during the melting process. Thus, when defect inclusion is at a lower level, the tensile properties of SLM and EBM specimens primarily depend on the microstructure instead of porosity. The $\alpha^{\prime}$ phase in SLM specimens result in a mixed brittle and ductile mode on the fracture surface, while the $\alpha+\beta$ microstructure of EBM specimen causes representative ductile 
failure in Ti-6Al-4V tensile specimens. However, increased defect inclusion has a negative impact on tensile strength, especially ductility. Cracks easily initiated and extended around these defects, resulting in a rapid failure.

SLM-produced Ti-6Al-4V specimens fabricated by Zone I and Zone II parameters show similar fatigue performance, due to a mutual influence of defects inclusion and martensitic microstructure. Thus, their fatigue data points present a scattered distribution on the stress-cycles graph. On the other hand, the fatigue strength displays a regular progressed plot with decreased cyclic stress when Zone III marginal parameters are used. Fatigue limit is seriously decreased due to lack of fusion.

At low porosity, EBM-produced Ti-6Al-4V fatigue specimens show a comparable fatigue performance to the optimally produced specimens. Their fracture surfaces display similar characteristics because defects are hard to avoid even if optimal processing parameters are used. Cracks always initiate at the boundaries of the $\alpha$ colonies or slip bands within the alpha lamellae, either at the surface or at the subsurface or defect site. When at a high porosity, the EBM fatigue specimens fail quickly under cyclic stress. 


\section{CHAPTER 6}

\section{CONCLUSIONS AND FUTURE WORK}

\subsection{Conclusions}

The research goal of this study was to establish a fundamental understanding of the effect of defects in parts made using SLM and EBM. Thus, the reasons of stochastic defects generation were analyzed. Porosity was correlated to processing parameters. Morphological characteristics and porosity of stochastic defects were analyzed using image processing method. Micro-CT was employed to non-destructively detect stochastic defects and estimate porosity using single slices and reconstructed 3D models of the SLM- and EBM-produced parts. With identified porosity, mechanical property tests of SLM- and EBM-produced Ti-6Al-4V specimens were carried out to characterize the effect of stochastic defects, and discuss the cracking mechanism with external load. The results achieved in this study give users of SLM and EBM equipment a better understanding of the characteristics of defects and their effects on the mechanical properties of SLM- and EBM-produced parts.

\subsubsection{The Effects of Processing Parameters on Defect Generation}

Defect generation was investigated by varying processing parameters from optimized parameters of SLM and EBM processes. A quantitative relationship between porosity and processing parameters was established through experiments. 
For SLM, a process window was introduced to show the effect of power and scan speed on laser melting Ti-6Al-4V powder. Processing parameter combinations are classified into "fully dense parameters" (Zone I), “over melting parameters" (Zone II), “incomplete melting parameters" (Zone III), and "over heating parameters" (Zone OH). Zone I parameters are capable of building fully dense parts with Ti-6Al-4V powder, although occasional defects were still seen in these otherwise "fully dense" parts. Zone II and III parameters, known as "marginal parameters," can be used to fabricate Ti-6Al-4V parts, but with stochastic defects. Parts may not be built using Zone OH parameters, because laser irradiation produces a large quantity of heat which fails to be conducted away immediately and the build fails. The accumulated strain energy results in serious deformation causing collision with the recoater blade.

According to the analysis of chapter 2 and 5, it was found that interior defects fabricated by Zone II marginal parameters are mainly attributed to gas bubbles entrapped or generated when a high laser energy is applied. Zone II stochastic defects often have a "stair" feature on their internal surface. Moreover, some defects may also be generated from the pits which result when the recoater blade removes welded particles from the solidified surface. However, gas bubble produced defects likely account for most Zone II defects in SLM parts, according to their morphological features and distribution. Stochastic defects are generated using Zone III marginal parameters due to insufficient laser energy input into the powder bed. Voids can be formed along or between hatch lines, where powder particles may be entrapped. Regions of incomplete melting site become the Zone III defect within SLM parts. 
For EBM, It was found that speed function index has the most significant influence on defect generation. However, speed function index is a reference number within the processing theme of the EBM system, and since the machine manufacturer does not provide information about how this factor is correlated with energy input, the exact mechanism for its effect is unknown. There is no apparent porosity variation by varying max current.

Both line offset and focus offset are important parameters resulting in defect generation. Overlap between hatch lines is reduced with increasing line offset. Porosity can be attributed to the un-melted powder under adjacent hatch lines. A further increased line offset value causes the melt pool to fail to contact the adjacent hatch line. Interstices between un-melted particles account for defect generation in the parts, while the deteriorated hatch lines exacerbate the inclusion of defects. Increasing the focus offset value results in a larger beam diameter during the EBM process. This leads to an increased beam spot and a lower energy density. The resulting increased overlap area and reduced melting depth creates an unstable melt pool, possibly due to thermal variations caused by un-melted powder entrapped below the melt pool. This unstable melt pool results in large voids on the surface.

\subsubsection{Defect Morphology in SLM and EBM specimens}

High porosity is usually accompanied by larger defects in both SLM and EBM specimens. Porosity can be estimated using image processing methods if cross section images are well processed. Defect morphology is related to marginal parameters. 
A Weibull distribution can be used to describe the histograms of contour characteristic values, such as circularity and elongation, of Zone II defects. The circularity value is very close to 1 , which means Zone II defects are circular or near-circular, which would be expected if these defects arise from entrapped gas bubbles. For elongation, the left skewness of the Weibull distribution and the convex features of the defect contours also demonstrate that Zone II defects have a spherical morphology. The histograms of circularity and elongation value of Zone III defects are normally distributed. This means that only a few defects have circular and symmetrical contours. Moreover, many Zone III defects show concave characteristics along their contours. Thus, most Zone III defects have an irregular morphology, which would be expected from defects arising from unmelted regions of powder.

Both line offset defects and focus offset defects in EBM parts are formed due to insufficient energy density, similarly to Zone III defects in SLM. But they are large enough to be observed by the naked eye. Their irregularity can be attributed to the large pores formed on each layer. A Weibull distribution can also be used to describe the histograms of circularity values of line offset defects and focus offset defects. But the left skewness implies that few defects have a circular contour. A certain amount of defects show concave characteristics. The histograms of elongation values are best described by the normal distribution. It can be deduced that long and narrow defects are generated in EBM specimens if line offset and focus offset are increased.

\subsubsection{Micro-CT Evaluation of Stochastic Defects}


Micro-CT is capable of detecting stochastic defects fabricated by SLM or EBM marginal parameters. However, the machine setup and CT detectability play an important role in defect visualization. Dense specimens usually display a homogeneous grayscale variation in $\mathrm{CT}$ scans. But stochastic defects cause irregular variations of brightness and darkness in localized areas of a single slice. The contour features of SLM stochastic defects are hard to see in CT scans due to their limited dimensions. But EBM stochastic defects are large enough to be clearly visualized.

The porosity of EBM specimens can also be estimated using single slices through image processing, while single slices of SLM specimens only show a rough texture and need advanced processing techniques for porosity analysis. Moreover, when stochastic defects are reconstructed into a 3D model, approximated porosity can be obtained by carefully selecting a neutralized grayscale threshold. Although its accuracy is limited compared to the Archimedes measurement, the capability of rapid estimation of porosity is promoted if a proper grayscale threshold is selected.

\subsubsection{Mechanical Properties of Ti-6Al-4V Parts with Defects}

The microstructure of SLM and EBM specimens primarily depend on cooling rate. Defect generation does not appear to influence microstructural evolution significantly during the solidification process. Thus, when defect inclusion is at a lower level, the tensile properties of SLM and EBM specimens primarily depend on the microstructure instead of porosity. The $\alpha^{\prime}$ phase in SLM specimens result in a mixed brittle and ductile mode of failure, as seen from the fracture surface, while the $\alpha+\beta$ microstructures in EBM specimens cause representative ductile failure. However, increased defect inclusion has a negative 
impact on tensile strength, especially ductility. Cracks easily initiate and extend from defects, resulting in a rapid failure.

SLM-produced Ti-6Al-4V specimens fabricated by Zone I and Zone II parameters show similar fatigue performance. Their fatigue data points present a scattered distribution on the stress-cycles graph. The fatigue limit is less than $350 \mathrm{MPa}$. This indicates that microstructure for this material has a more important role on its fatigue performance than the presence of spherical defects. For Zone III defects, the fatigue strength displays a regular plot with decreased cyclic stress when Zone III marginal parameters are used. The fatigue limit of Zone III defects is very low (around $100 \mathrm{MPa}$ ) due to lack of fusion.

At low porosity, EBM-produced Ti-6Al-4V fatigue specimens show a comparable fatigue performance to the optimally produced specimens. Their fracture surfaces display similar characteristics because defects are hard to avoid even if optimal processing parameters are used. The fatigue limit is around $450 \mathrm{MPa}$. Cracks always initiate at the boundaries of the $\alpha$ colonies or slip bands within the alpha lamellae, either at the surface or at the subsurface or at a defect site. When at a higher porosity, EBM fatigue specimens fail quickly under cyclic stress, and the fatigue limit is significantly reduced to $50 \mathrm{MPa}$.

In conclusion, this work has resulted in a better understanding of porosity formation and their effects on part performance for SLM and EBM produced specimens. This work illustrates that although mechanical properties are typically degraded to some degree by the presence of porosity, marginal parameters may be desirable for some applications where porosity is beneficial and the reduction of mechanical properties is not detrimental.

\subsection{Future Work}


The current work identified numerous future research directions for stochastic defects generated in SLM- and EBM-produced Ti-6Al-4V parts:

- The formation mechanism of Zone II defects of SLM parts can be further analyzed to identify whether their spherical morphology is caused by entrapped inert gas, vaporized material, or other mechanisms. Elemental analysis could be conducted on the surface of the spherical defects. After comparing the variation in elemental composition between the surface of these defects with the bulk composition and the pre-alloyed Ti-6Al-4V powder, the process of Zone II defect formation could be determined.

- Advanced image processing techniques could be employed to process single slices of CT scans to determine whether there is a correlation between porosity and grayscale distribution for pores which are smaller than the voxel size of the scan. This could be especially useful for SLM-produced parts with small stochastic defects. If successful, single slices could be used to accurately estimate porosity without destructive treatments to the parts.

- Image correction methods should be applied to reconstructed 3D models. This should enable the reduction of radiodensity inconsistency to the point where accurate estimation of 3D porosity and morphology could be obtained from CT scans.

- Besides tensile and fatigue performance, other physical and mechanical property tests may be conducted to clearly understand the effects of stochastic defect on SLM- and EBM-produced parts, such as thermal conductivity, electrical conductivity, hardness, and other fracture modes. 
- The intentional use of marginal parameters for various applications is a promising research direction. Porous parts fabricated using SLM or EBM marginal parameters could be utilized in the same way as powder metallurgy based porous parts for filtration, flow control and permeability. Porous medical implants which enable tissue in-growth could also be fabricated using marginal parameters due to the ability of SLM and EBM to make complex structures. 


\section{REFERENCES}

Akhtar, S., Wright, C.S., Youseffi, M., Hauser, C., Childs, T.H.C., Taylor, C.M., Baddrossamay, M., Xie, J., Fox, P., O’Neill, W. (2003). Direct selective laser sintering of tool steel powders to high density: Part B-The effect on microstructural evolution. Solid Freeform Fabrication Symposium, 656-667.

Amato, K.N., Gaytan, S.M., Murr, L.E., Martinez, E., Shindo, P.W., Hernandez, J., Collins, S., Medina, F. (2012). Microstructures and mechanical behavior of Inconel 718 fabricated by selective laser melting. Acta Materialia, 60(5), 2229-2239.

----. (2012). Standard Terminology for Additive Manufacturing Technologies (F2792-12). American Society for Testing and Materials (ASTM). http://www.astm.org.

Badrossamay, M., Childs, T.H.C. (2007). Further studies in selective laser melting of stainless and tool steel powders. International Journal of Machine Tools \& Manufacture, 47(5), 779-784.

Biamino, S., Penna, A., Ackelid, U., Sabbadini, S., Tassa, O., Fino, P., Pavese, M., Gennaro, P., Badini, C. (2011). Electron beam melting of Ti-48Al-2Cr-2Nb alloy: Microstructure and mechanical properties investigation. Intermetallics, 19(6), 776781.

Bourell, D., Wohlert, M., Harlan, N., Das, S., Beaman, J. (2002). Powder densification maps in selective laser sintering. Advanced Engineering Materials, 4(9), 663-669.

Brown S., Bailey, D., Willowson, K, Baldock, C. (2008). Investigation of the relationship between linear attenuation coefficients and CT Hounsfield units using radionuclides for SPECT. Applied Radiation and Isotopes, 66(9), 1206-1212.

Campbell, I., Bourell, D., Gibson, I. (2012). Additive manufacturing: Rapid prototyping comes of age. Rapid Prototyping Journal, 18(4), 255-258.

Carvalho, A.A., Rebello, J.M.A., Souza, M.P.V., Sagrilo, L.V.S., Soares, S.D. (2008). Reliability of non-destructive test techniques in the inspection of pipelines used in the oil industry. International Journal of Pressure Vessels and Piping, 85(11), 745-751. 
Chassignole, B., El Guerjouma, R., Ploix, M., Fouquet, T. (2010). Ultrasonic and structural characterization of anisotropic austenitic stainless steel welds: Towards a higher reliability in ultrasonic non-destructive testing. NDT\&E International, 43(4), 273-282.

Childs, T.H.C. and Hauser, C. (2005a). Raster scan selective laser melting of the surface layer of a tool steel powder bed. Proceedings of the Institution of Mechanical Engineers, Part B: Journal of Engineering Manufacture. 219B, 379-384.

Childs, T.H.C., Hauser, C., Badrossamay, M. (2005b). Selective laser sintering (melting) of stainless and tool steel powders: experiments and modeling. Proceedings of the Institution of Mechanical Engineers, Part B: Journal of Engineering Manufacture. 219B, 339-358.

Cormier, D., Harrysson, O., West, H. (2003). Characterization of high alloy steel produced via electron beam melting. Solid Freeform Fabrication Symposium. 548-558.

Cormier, D., Harrysson, O., West, H. (2004a). Characterization of H13steel produced via electron beam melting. Rapid Prototyping Journal, 10(1), 35-41.

Cormier, D., West, H., Harrysson, O. (2004b). Characterization of thin walled Ti-6Al-4V components produced via electron beam melting. Solid Freeform Fabrication Symposium, 440-447.

Das, S. (2001a). On some physical aspects of process control in direct selective laser sintering of metals-Part I. Solid Freeform Fabrication Symposium. 85-93.

Das, S. (2001b). On some physical aspects of process control in direct selective laser sintering of metals-Part II. Solid Freeform Fabrication Symposium. 94-101.

Das, S. (2001c). On some physical aspects of process control in direct selective laser sintering of metals-Part III. Solid Freeform Fabrication Symposium. 102-109.

Das, S. (2003). Physical aspects of process control in selective laser sintering of metals. Advanced Engineering Materials, 5(10), 701-711.

Dieter, G.E. (1986). Mechanical Metallurgy. McGraw Hill Co. New York.

Dutta Majumdar, J., Pinkerton, A., Liu, Z., Manna, I., Li, L. (2005). Microstructure characterisation and process optimization of laser assisted rapid fabrication of $316 \mathrm{~L}$ stainless steel. Applied Surface Science, 247(1-4), 320-327.

Facchini, L., Magalini, E., Robotti, P., Molinari, A. (2009). Microstructure and mechanical properties of Ti-6Al-4V produced by electron beam melting of pre-alloyed powders. Rapid Prototyping Journal, 15(3), 171-178. 
Facchini, L., Magalini, E., Robotti, P., Molinari, A., Höges, S.; Wissenbach, K. (2010a). Ductility of a Ti-6Al-4V alloy produced by selective laser melting of prealloyed powders. Rapid Prototyping Journal, 16(6), 450-459.

Facchini, L., Vicente, N., Lonardelli, I., Magalini, E., Robotti, P., Molinari, A. (2010b). Metastable austenite in 17-4 precipitation-hardening stainless steel produced by selective laser melting. Advanced Engineering Materials, 12(3), 184-188.

Filip, R., Kubiak, K., Ziaja, W., Sieniawski, J. (2003). The effect of microstructure on the mechanical properties of two-phase titanium alloys. Journal of Materials Processing Technology, 133(1-2), 84-89.

Fischer, P., Romano, V., Weber, H.P., Karapatis, N.P., Boillat, E., Glardon, R. (2003). Sintering of commercially pure titanium powder with a Nd:YAG laser source. Acta Materialia, 51(6), 1651-1662.

Fukuda, H., Takahashi, H., Kuramoto, K., Nakano, T. (2012). Effect of energy density of incident beam on mechanical property of titanium alloy products fabricated by electron beam melting (EBM) method. Materials Science Forum, 706-709, 488-491.

Gaytan, S.M., Murr, L.E., Martinez, E. (2010). Comparison of microstructures and mechanical properties for solid and mesh cobalt-base alloy prototypes fabricated by electron beam melting. Metallurgical and Materials Transactions A (Physical Metallurgy and Materials Science), 41(12), 3216-3227.

Gaytan, S.M., Murr, L.E., Medina, F., Martinez, E., Lopez, M.I., Wicker, R.B. (2009). Advanced metal powder based manufacturing of complex components by electron beam melting. Materials Technology, 24(3), 180-190.

Gibson, I., Rosen, D.W., Stucker, B. (2009). Additive Manufacturing Technologies: Rapid Prototyping to Direct Digital Manufacturing. Springer, New York, NY.

Gil Mur, F.X., Rodriguez, D., Planell, J.A. (1996). Influence of tempering temperature and time on the $\alpha^{\prime}-\mathrm{Ti}-6 \mathrm{Al}-4 \mathrm{~V}$ martensite. Journal of Alloys and Compounds, 234(2), 287289.

Gusarov, A. V., Yadroitsev, I., Bertrand, P., Smurov, I. (2009). Model of radiation and heat transfer in laser-powder interaction zone at selective laser melting. Journal of Heat Transfer, 131(7), 072101-072110.

Hauser, C., Childs, T.H.C., Dalgamo, K.W. (1999a). Selective laser sintering of stainless steel $314 \mathrm{~S}$ HC processed using room temperature powder beds. Solid Freeform Fabrication Symposium. 273-280. 
Hauser, C., Childs, T.H.C., Dalgarno, K.W., Eane, R.B. (1999b). Atmospheric control during direct selective laser sintering of stainless steel 314 S powder. Solid Freeform Fabrication Symposium. 265-272.

Hauser, C., Childs, T.H.C., Taylor, C.M., Badrossamay, M., Akhtar, S., Wright, C.S., Youseffi, M., Xie, J., Fox, P., O’Neill, W. (2003). Direct selective laser sintering of tool steel powders to high density: Part A-effects of laser beam width and scan strategy. Solid Freeform Fabrication Symposium, 644-655.

Hiemenz, J. (2007). Electron beam melting. Advanced Materials and Processes. 165(3), 45-46.

Hollander, D., Walter, M., Wirtz, T., Sellei, R., Schmidt-Rohlfing, B., Paar, O., Erli, H.-J. (2006). Structural, mechanical and in vitro characterization of individually structured Ti-6Al-4V produced by direct laser forming. Biomaterials, 27(7), 955-963.

Hung, Y.Y., Chen, Y.S., Ng, S.P., Liu, L., Huang, Y.H., Luk, B.L., Ip, R.W.L., Wu, C.M.L., Chung, P.S. (2009). Review and comparison of shearography and active thermography for nondestructive evaluation. Materials Science and Engineering R, 64(5-6), 73-112.

Ivasishin, O. M., Teliovich, R. V. (1999). Potential of rapid heat treatment of titanium alloys and steels. Materials Science and Engineering, 263(2), 142-154.

Kahnert, M., Lutzmann, S., Zaeh, M. F. (2007). Layer formations in electron beam sintering. Solid Freeform Fabrication Symposium, 88-99.

Kim, W.H., Laird, C. (1978). Crack Nucleation and State I Propagation in High Strain Fatigue-II. Mechanism. Acta Metallurgica, 26(5), 789-799.

Kircher, R.S., Christensen, A.M., Wurth, K.W. (2009). Electron beam melted (EBM) CoCr-Mo alloy for orthopaedic implant applications, Solid Freeform Fabrication Symposium, 428-436.

Koike, M., Greer, P., Owen, K., Lilly, G., Murr, L.E., Gaytan S.M., Martinez, E., Okabe, T. (2011). Evaluation of titanium alloys fabricated using rapid prototyping technologies-electron beam melting and laser beam melting, Materials, 4, 1776-1792.

Kong, C., Tuck, C.J., Ashcroft, I.A., Wildman R.D., Hague R. (2011). High density Ti6Al4V via SLM processing: microstructure and mechanical properties. Solid Freeform Fabrication Symposium, 475-483.

Kruth, J.P., Froyen, L., Rombouts, M., Van Vaerenbergh, J., Mercelis, P. (2003). New ferro powder for selective laser sintering of dense parts. CIRP Annals - Manufacturing Technology, 52(1), 139-142. 
Kruth, J.P., Froyen, L., Van Vaerenbergh, J. (2004). Selective laser melting of iron-based powder. Journal of Materials Processing Technology, 149(1-3), 616-622.

Kruth, J., Vandenbroucke, B., Van Vaerenbergh, J. (2005a). Rapid Manufacturing of Dental Prostheses by means of Selective Laser Sintering/Melting. Proceedings of the $A F P R, \mathrm{~S} 4$.

Kruth J.P., Mercelis P., Van Vaerenbergh J. (2005b). Binding mechanisms in selective lasersintering and selective laser melting. Rapid Prototyping Journal, 11(1), 26-36.

Kruth, J.P., Levy, G., Klocke, F., Childs, T.H.C. (2007). Consolidation phenomena in laser and powder-bed based layered manufacturing. Annals of the CIRP, 56(2), 730-759.

Kubiak, K., Sieniawski, J. (1998). Development of the microstructure and fatigue strength of two phase titanium alloys in the processes of forging and heat treatment. Journal of Materials Processing Technology, 78, 117-121.

Kumar, S. (2008a). Microstructure and wear of SLM materials. Solid Freeform Fabrication Symposium, 128-142.

Kumar, S., Kruth, J.P. (2008b). Wear performance of SLS/SLM materials. Advanced Engineering Materials, 10(8), 750-753.

Levy, G., Schindel, R., Kruth. J.P. (2003). Rapid manufacturing and rapid tooling with layer manufacturing (LM) technologies, state of the art and future perspectives. CIRP Annals-Manufacturing Technology, 52(2), 589-609.

Li, L. (2006). Repair of directionally solidified superalloy GTD-111 by laser-engineered net shaping. Journal of Materials Science, 41(23), 7886-7893.

Li, R., Liu, J., Shi, Y., Du, M., Xie, Z., (2010). 316L stainless steel with gradient porosity fabricated by selective laser melting. Journal of Materials Engineering and Performance, 19(5), 666-671.

Li, S.J., Murr, L.E., Cheng, X.Y., Zhang, Z.B., Hao, Y.L., Yang, R., Medina, F., Wicker, R.B. (2012). Compression fatigue behavior of Ti-6Al-4V mesh arrays fabricated by electron beam melting. Acta Materialia, 60(3), 793-802.

Lu, W., Lin, F., Han, J., Qi, H, Yan, N. (2009). Scan strategy in electron beam selective melting. Tsinghua Science and Technology, 14(S1), 120-126.

Malinov, S., Sha, W., Guo, Z., Tang, C.C., Long, A.E. (2002). Synchrotron X-ray diffraction study of the phase transformations in titanium alloys. Materials Characterization, 48(4), 279-295. 
Meiners, W., Wissenbach, K., Poprawe, R. (1998). Direct generation of metal parts and tools by selective laser powder remelting (SLPR). Proceedings of the Laser Materials Processing Conference ICALEO'98, 2, 31-37.

Mercelis, P. and Kruth, J. P. (2006). Residual stresses in selective laser sintering and selective laser melting. Rapid Prototyping Journal, 12(5), 254-265.

Morgan, R. H., Papworth, A. J., Sutcliffe C., Fox, P., O'Neill, W. (2002). High density net shape components by direct laser re-melting of single-phase powders. Journal of Materials Science, 37(15), 3093- 3100.

Morgan, R., Sutcliffe, C.J., O'Neill, W. (2001). Experimental investigation of nanosecond pulsed Nd:YAG laser re-melted pre-placed powder beds. Rapid Prototyping Journal, 7(3), 159-172.

Morgan, R., Sutcliffe, C. J., O' Neill, W. (2004). Density analysis of direct metal laser remelted 316L stainless steel cubic primitives. Journal of Materials Science, 39(4), 1195-1205.

Mumtaz, K. and Hopkinson N. (2010). Selective laser melting of Inconel 625 using pulse shaping. Rapid Prototyping Journal, 16(4), 248-257.

Murr, L.E., Esquivel, E.V., Quinones, S.A., Gaytan, S.M., Lopez, M.I., Martinez, E.Y., Medina, F., Hernandez, D.H., Martinez, E., Martinez, J.L., Stafford, S.W., Brown, D.K., Hoppe, T., Meyers, W., Lindhe, U., Wicker, R.B. (2009a). Microstructures and mechanical properties of electron beam-rapid manufactured Ti-6Al-4V biomedical prototypes compared to wrought Ti-6Al-4V. Materials Characterization, 60(2), 96105.

Murr, L.E., Quinones, S.A., Gaytan, S.M., Lopez, M.I., Rodela, A., Martinez, E.Y., Hernandez, D.H., Martinez, E., Medina, F., Wicker, R.B. (2009b). Microstructure and mechanical behavior of Ti-6Al-4V produced by rapid-layer manufacturing, for biomedical applications. Journal of the mechanical behavior of biomedical materials, 2(1), 20-32.

Murr, L. E., Gaytan, S. M., Medina, F., Martinez, E., Hernandez, D.H., Martinez, L., Lopez, M.I., Wicker, R.B., Collins, S. (2009c). Effect of build parameters and build geometries on residual microstructures and mechanical properties of Ti-6Al-4V components built by electron beam melting (EBM). Solid Freeform Fabrication Symposium. 374-397.

Murr, L.E., Gaytan, S.M., Medina, F., Martinez, E., Martinez, J.L., Hernandez, D.H., Machado, B.I., Ramirez, D.A., Wicker, R.B. (2010). Characterization of Ti-6Al-4V open cellular foams fabricated by additive manufacturing using electron beam melting. Materials Science and Engineering A, 527(7-8), 1861-1868. 
Murr, L.E., Martinez, E., Gaytan, S.M., Ramirez, D.A., MacHado, B.I., Shindo, P.W., Martinez, J.L., Medina, F., Wooten, J., Ciscel, D., Ackelid, U., Wicker, R.B. (2011). Microstructural architecture, microstructures, and mechanical properties for a nickelbase superalloy fabricated by electron beam melting. Metallurgical and Materials Transactions A: Physical Metallurgy and Materials Science, 42(11), 3491-3508.

Murr, L.E., Gaytan, S.M., Ramirez, D.A., Martinez, E., Hernandez, J., Amato, K.N., Shindo, P.W., Medina, F.R., Wicker, R.B. (2012). Metal fabrication by additive manufacturing using laser and electron beam melting technologies. Journal of Materials Science and Technology, 28(1), 1-14.

Niu, H.J. and Chang, I.T.H. (1999a). Instability of scan tracks of selective laser sintering of high speed steel powder. Scripta Materialia, 41(11), 1229-1234.

Niu, H.J., Chang, I.T.H. (1999b). Selective laser sintering of gas and water atomized high speed steel powders. Scripta Materialia, 41(1), 25-30.

Niu, H. J., Chang, I. T. H. (2000). Selective laser sintering of gas atomized M2 high speed steel powder. Journal of Materials Science, 35(1), 31-38.

Parthasarathy, J., Starly, B., Ramana, S., Christensen, A. (2010). Mechanical evaluation of porous titanium (Ti6A14V) structures with electron beam melting (EBM). Journal of the Mechanical Behavior of Biomedical Materials, 3(3), 249-259.

Rafi, H.K., Karthik, N.V., Gong, H., Starr, T, Stucker, B. (2013). Microstructures and Mechanical Properties of Ti6Al4V Parts Fabricated by Selective Laser Melting and Electron Beam Melting. Journal of Materials Engineering and Performance.

Ramirez, D.A., Murr, L.E., Li, S.J., Tian, Y.X., Martinez, E., Martinez, J.L., Machado, B.I., Gaytan, S.M., Medina, F., Wicker, R.B. (2011a). Open-cellular copper structures fabricated by additive manufacturing using electron beam melting. Materials Science and Engineering A, 528(16-17), 5379-5386.

Ramirez, D.A., Murr, L.E., Martinez, E., Hernandez, D.H., Martinez, J.L., Machado, B.I., Medina, F., Frigola, P., Wicker, R.B. (2011b). Novel precipitate-microstructural architecture developed in the fabrication of solid copper components by additive manufacturing using electron beam melting. Acta Materialia, 59(10), 4088-4099.

Ramosoeu, M.K.E., Booysen, G., Ngonda, T.N., Chikwanda, H.K. (2011). Mechanical properties of direct laser sintered Ti-6Al-4V. Materials Science and Technology Conference (MS\&T), 1460-1468.

Sam Froes, F.H. (2012). Titanium powder metallurgy: a review-part 2. Advanced Materials \& Processes, 170(10), 26-29. 
Santos, E., Abe, F., Kitamura, Y., Shiomi, M. (2002). Mechanical properties of pure titanium models processed by selective laser melting. Solid Freeform Fabrication Symposium. 180-186.

Schwerdtfeger, J., Singer, R. F., Ko“rner, C. (2012). In situ flaw detection by IR-imaging during electron beam melting. Rapid Prototyping Journal, 18(4), 259-263.

Sercombe, T., Jones, N., Day, R., Kop, A. (2008). Heat treatment of Ti-6Al-7Nb components produced by selective laser melting. Rapid Prototyping Journal, 14(5), 300-304.

Shiomil, M., Osakadal, K., Nakamura, K., Yamashita, T., Abe, F. (2004). Residual stress within metallic model made by selective laser melting process. CIRP Annals Manufacturing Technology, 53(1), 195-198.

Sieniawski, J., Filip, R., Ziaja, W. (1997). The effect of microstructure on the mechanical properties of two-phase titanium alloys. Materials \& Design, 18, 361-363.

Simchi, A. (2006). Direct laser sintering of metal powders: mechanism, kinetics and microstructural features. Materials Science and Engineering A, 428(1-2), 148-158.

Soboyejo, W., Srivatsan, T. (2006). Advanced Structural Materials: Properties, Design Optimization, and Applications. CRC Press, 359-400.

Simchi, A., Pohl, H. (2003). Effects of laser sintering processing parameters on the microstructure and densification of iron powder. Materials and Engineering $A$ (Structural Materials: Properties, Microstructure and Processing), A359(1-2), 119128.

Song, Y. (1997). Experimental study of the basic process mechanism for direct selective laser sintering of low-melting metallic powder. CIRP Annals-Manufacturing Technology, 46(1), 127-130.

Spierings, A. B., Levy, G. (2009). Comparison of density of stainless steel 316L parts produced with selective laser melting using different powder grades. Solid Freeform Fabrication Symposium. 342-353.

Spierings, A.B., Schneider, M. (2011). Comparison of density measurement techniques for additive manufactured metallic parts. Rapid Prototyping Journal, 17(5), 380-386.

Thijs, L., Verhaeghe, F., Craeghs T., Van Humbeeck, J., Kruth, J.P. (2010). A study of the microstructural evolution during selective laser melting of Ti-6Al-4V. Acta Materialia, 58(9), 3303-3312.

Tolochko, N., Mozzharov, S., Yadroitsev, I. (2004). Balling processes during selective laser treatment of powders. Rapid Prototyping Journal, 10(2), 78-87. 
Van Bael, S., Kerckhofs, G., Moesen, M., Pyka, G., Schrooten, J., Kruth, J.P. (2011). Micro-CT-based improvement of geometrical and mechanical controllability of selective laser melted Ti6A14V porous structures. Materials Science and Engineering A (Structural Materials: Properties, Microstructure and Processing), 528(24), 74237431.

Vandenbroucke, B., Kruth J.P. (2007). Selective laser melting of biocompatible metals for rapid manufacturing of medical parts. Rapid Prototyping Journal, 13(4), 196-203.

Verhaeghe, F., Craeghs, T., Heulens, J., Pandelaers, L. (2009). A pragmatic model for selective laser melting with evaporation. Acta Materialia, 57(20), 6006-6012.

Von Allmen, M. (1987). Laser-beam Interactions with Materials. Springer-Verlag, Berlin, 146-174.

$\mathrm{Xu}, \mathrm{P}$. (2008). Eddy current testing probe composed of double uneven step distributing coils for crack detection. PhD dissertation, Saga University.

Yadroitsev, I., Bertrand, Ph., Smurov, I. (2007a). Parametric analysis of the selective laser melting process. Applied Surface Science, 253(19), 8064-8069.

Yadroitsev, I., Pavlov, M., Bertrand, Ph., Smurov, I. (2009). Mechanical properties of samples fabricated by selective laser melting, 14èmes Assises Européennes du Prototypage\& Fabrication Rapide. Paris.

Yadroitsev, I., Smurov, I. (2011). Surface morphology in selective laser melting of metal powders. Physics Procedia, 12, 264-270.

Yadroitsev, I., Thivillon, L., Bertrand, P., Smurov, I. (2007b). Strategy of manufacturing components with designed internal structure by selective laser melting of metallic powder. Applied Surface Science, 254(4), 980-983.

Yasa, E., Deckers, J., Craeghs, T., Badrossamay, M., Kruth, J.P. (2009). Investigation on Occurrence of Elevated Edges in Selective Laser Melting. Solid Freeform Fabrication Symposium. 180-192.

Yasa, E., Deckers, J., Kruth, J.P. (2011). The investigation of the influence of laser remelting on density, surface quality and microstructure of selective laser melting parts. Rapid Prototyping Journal, 17(5), 312-327.

Zäh, M. F., Lutzmann, S. (2010). Modelling and simulation of electron beam melting. Production Engineering. 4(1), 15-23. 
APPENDICES 


\begin{abstract}
APPENDIX A
DETECTION TO DETERMINISTIC DEFECTS IN SLM-PRODUCED PARTS VIA DESTRUCTIVE CHARACTERIZATION TECHNIQUE AND NON-DESTRUCTIVE TESTING METHODS
\end{abstract}

This is a technical report to the project investigators

\begin{abstract}
A.1 Purpose
This preliminary test was conducted for investigating the formability and detectability of deterministic defects in Selective Laser Melting (SLM) parts. Destructive characterization technique and Non-Destructive Testing (NDT) methods were used in this test. Deterministic defects refer to the defects which are designed in a CAD file and fabricated deliberately using Additive Manufacturing (AM) methods. Due to the structure flexibility of AM-produced parts, deterministic defects can be directly entrapped inside and formed to an approximate geometry as designed. Specimens were all fabricated using SLM method in this test, which is a typical Powder Bed Fusion (PBF) based AM method [1].
\end{abstract}

\title{
A.2 Sample Fabrication
}

Specimen with deterministic defects was designed and modeled using Solidworks software package. The CAD model was then converted to STL file and loaded into Materialise Magics (Version 16.0) software package for adjusting orientation and adding support structures. After that, the modified STL file were sliced at $20 \mu \mathrm{m}$ layer thickness 
by SLIview software and saved as SLI file, which was used in an EOS M270 Direct Metal Laser Sintering (DMLS) system. EOS maraging steel powder was used to manufacture two identical specimens with factory default processing parameters. The chamber of EOS M270 system was filled with nitrogen gas to avoid oxidization.

The CAD file and an as-built SLM specimen is shown in Figure A.1. Cylindrical and conical defects are distributed at two levels which represent two different distances from the bottom surface. Geometric features are illustrated in the figure.
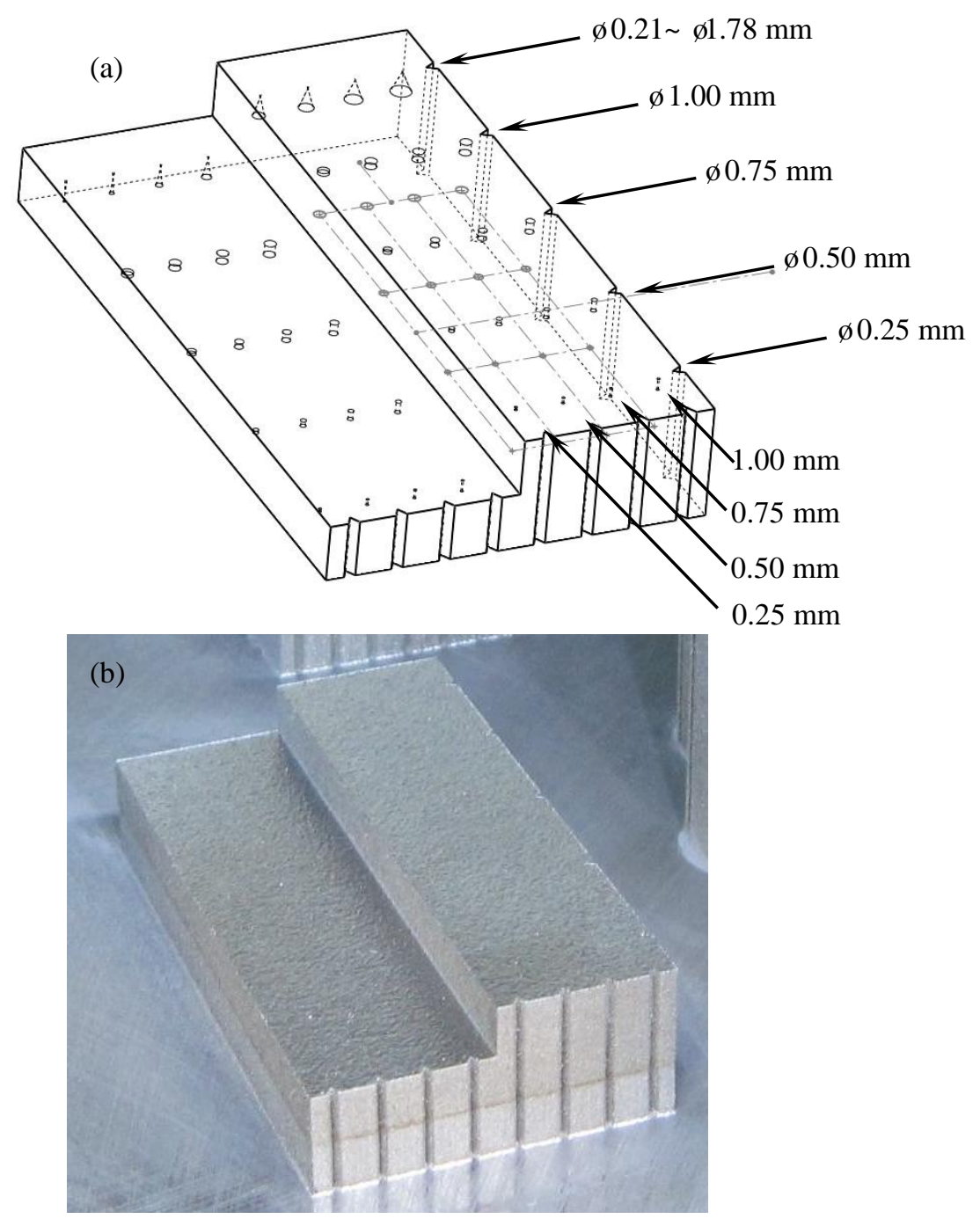

Figure A.1. SLM-produced specimen for NDT. (a) CAD file; (b) As-built specimen. 


\section{A.3 Defects Detection via Destructive Characterization Technique}

The first specimen was sectioned vertically to characterize defects. As shown in Table A.1, it was noted that the minimum designed cylindrical defect $(\Phi 0.25 \mathrm{~mm}$ in diameter and $0.25 \mathrm{~mm}$ in height) could be formed and located in the SLM process, although its cylindrical morphology is deteriorated. It means that the minimum structural characteristic of SLM parts could be less than $0.25 \mathrm{~mm}$. Likewise, any defects with horizontal or vertical dimension larger than $0.25 \mathrm{~mm}$ could be formed.

Table A.1. Formability of Cylindrical Defects in the SLM-Produced Specimen

\begin{tabular}{c|c|c|c|c}
\hline \hline & $0.25 \mathrm{~mm}$ & $0.50 \mathrm{~mm}$ & $0.75 \mathrm{~mm}$ & $1.00 \mathrm{~mm}$ \\
\hline$\varnothing 1.00 \mathrm{~mm}$ & $\sqrt{ }$ & $\sqrt{ }$ & $\sqrt{ }$ & $\sqrt{ }$ \\
\hline$\varnothing 0.75 \mathrm{~mm}$ & $\sqrt{ }$ & $\sqrt{ }$ & $\sqrt{ }$ & $\sqrt{ }$ \\
\hline$\varnothing 0.50 \mathrm{~mm}$ & $\sqrt{ }$ & $\sqrt{ }$ & $\sqrt{ }$ & $\sqrt{ }$ \\
\hline$\varnothing 0.25 \mathrm{~mm}$ & $\sqrt{ }$ & $\sqrt{ }$ & $\sqrt{ }$ & $\sqrt{ }$ \\
\hline
\end{tabular}

All the conical defects have the same height $(2 \mathrm{~mm})$, but different opening angles ranging from $6^{\circ}$ to $48^{\circ}$ at the interval $6^{\circ}$. An increasing opening angle corresponds to an increasing bottom diameter and an apparent overhang structure. The conical defect with the opening angle of $6^{\circ}$ has the minimum bottom diameter $(\varnothing 0.21 \mathrm{~mm})$. After being sectioned, most of conical defects could be detected except the defect with bottom diameter $0.21 \mathrm{~mm}$ (Table A.2). Based on this result, it is assumed that the minimum feature dimension should be larger than $0.21 \mathrm{~mm}$ to be formed during the SLM process.

Table A.2. Formability of Conical Defects in the SLM-Produced Specimen

\begin{tabular}{c|c|c|c|c|c|c|c|c}
\hline & $\varnothing 1.78$ & $\varnothing 1.54$ & $\varnothing 1.30$ & $\varnothing 1.07$ & $\varnothing 0.84$ & $\varnothing 0.63$ & $\varnothing 0.41$ & $\varnothing 0.21$ \\
\hline $2 \mathrm{~mm}$ & $\sqrt{ }$ & $\sqrt{ }$ & $\sqrt{ }$ & $\sqrt{ }$ & $\sqrt{ }$ & $\sqrt{ }$ & $\sqrt{ }$ & $\times$ \\
\hline
\end{tabular}




\section{A.4 Defects Detection via NDT Methods}

Attempts were performed to detect deterministic defects by three commonly used NDT methods: thermography testing, ultrasonic testing, and X-ray computed tomography (CT) testing.

\section{A.4.1 Thermography}

Flash thermography was not used for testing because the deterministic defects of metallic specimen were too far from the surface. Thermal energy might not be able to be transferred to the interior of specimen. Thus, vibrothermography was conducted to the second specimen at Center for Nondestructive Evaluation of Iowa State University. The specimen was vibrated at flexural resonances using a piezostack transducer. Frequency sweep was firstly carried out for searching the resonance frequency in the spectrum. Then, sinusoidal excitation was applied at each resonance frequency to the specimen, such as $20.4 \mathrm{kHz}, 21.5 \mathrm{kHz}, 27.4 \mathrm{kHz}$ and $42.6 \mathrm{kHz}$. But, unfortunately, these deterministic defects could not be detected by IR camera, even if at some lower frequencies.

Generally, heat generation in cracks or defects is generated from one or a combination of three mechanisms: friction, plasticity, and viscoelasticity [2]. Frictional heat generation occurs due to rubbing surface of cracks or defects. Frictional rubbing may also cause plastic deformation along crack surfaces. Viscoelastic heating is resulted by bulk heating of the structure due to vibrational stress, and localized heating at regions of stress concentration. Because the deterministic defects were voids in the specimen. Only unmelted powders were entrapped and no rubbing surfaces were formed. Frictional heat could not occur in the vibrothermography since there was no contacting surface available to rub. 
It was inferred that vibrational stress did not cause viscoelastic heating or localized heating either. Thus, the deterministic defects failed to be detected by the vibrothermography.

\section{A.4.2 Ultrasonic Testing (UT)}

Ultrasonic Testing was carried out on a specimen with deterministic defects. The UT setup consists of a 3-12 GHz A/D board, a JSR Pulser and receiver, ODIS V3.2 software, $20 \mathrm{MHz}$ immersion transducers, as shown in Figure A.2. Longitudinal ultrasonic signals was transmitted to the specimen through fluid medium. When there is a defect in the wave path, part of the energy will be reflected back from the defect surface. The reflected wave signal is received by the transducer and transformed into electrical signal to be displayed.

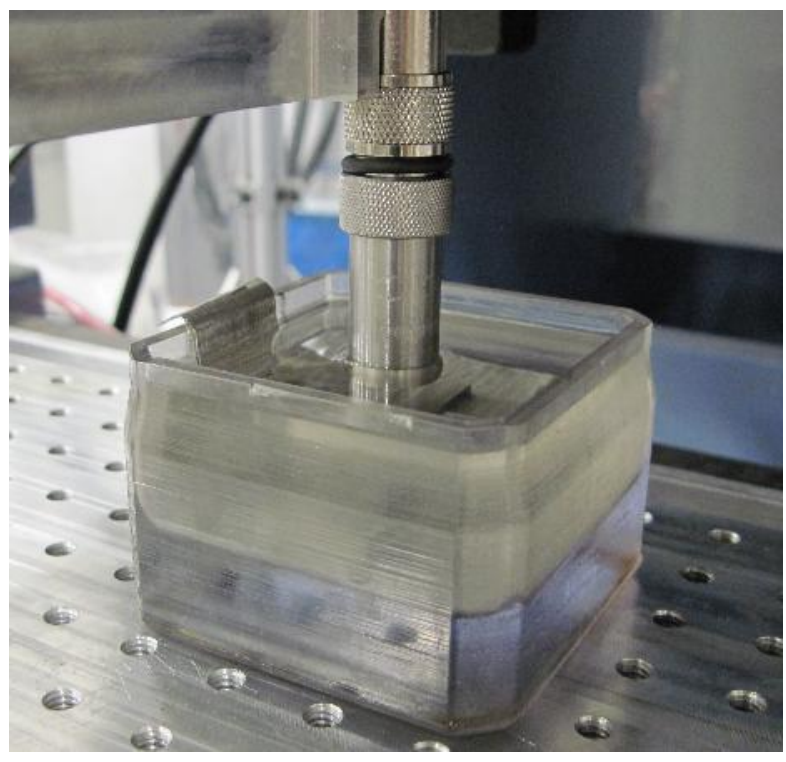

Figure A.2. UT transducer.

When ultrasonic waves travel into a sample, part of the energy can be reflected back. Multiple reflections from the back surface are known as back wall echoes. Figure A.3 show back wall echoes by A-scan. A display of the received pulse amplitude is 
represented as a displacement along vertical axis and the travel time of the ultrasonic pulse is represented as a displacement along horizontal axis.

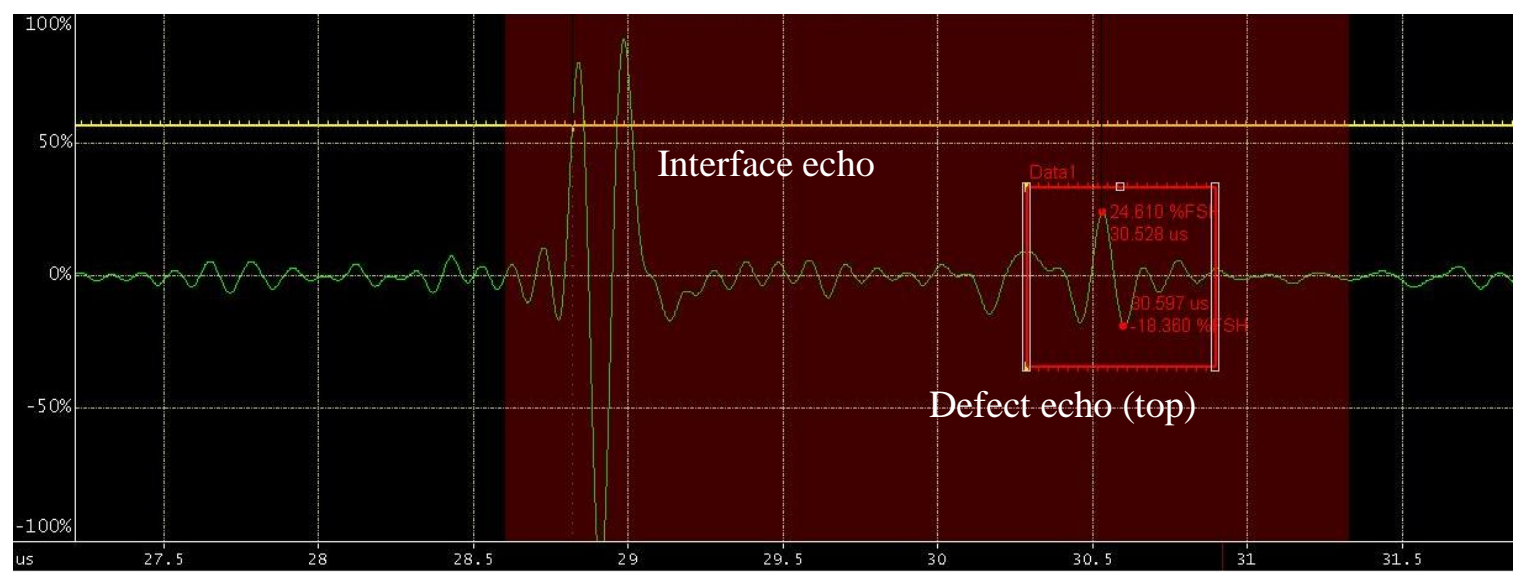

Figure A.3. UT A-scan result.

The defect can be successfully detected by the A scan of UT. The scan result indicates the depth and the amplitude of the sound reflections from the defect.

\section{A.4.3 X-ray Computed Tomography (CT)}

CT scanning was carried out using a micro-CT scanner (Model ACTIS 200/225 Ffi-HR CT/DR system. BIR Inc., Chicago, IL with built-in X-ray system FXE 225.20, Fein Focus U.S.A) in the orthopaedic bioengineering laboratory at University of Louisville. Due to the limited turnable stage, large specimen was hardly mounted in the micro-CT machine. In order to achieve a higher resolution, two SLM-produced fatigue specimens (Ti-6Al-4V) were scanned instead of the specimens used for thermography and ultrasonic testing. The fatigue specimens were built with single deterministic defect in the center of gage section, as shown in Figure A.4. Each fatigue specimen was scanned individually with a certain amount of single slices. Slices were created $7 \mu \mathrm{m}$ apart from each other. Each pixel of the single slice has a dimension of $7 \times 7 \mu \mathrm{m}$. Thus, the voxel in these slices is $7 \times 7 \times 7 \mu \mathrm{m}$. 

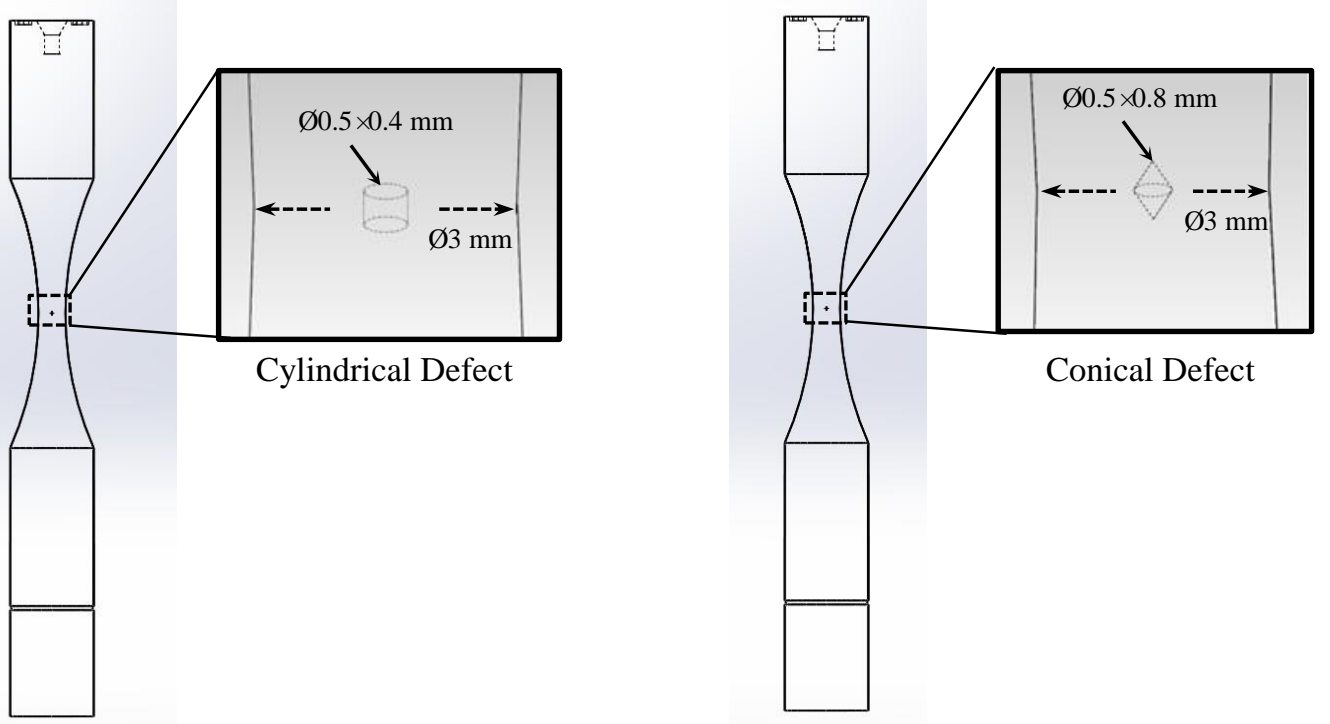

Figure A.4. SLM-produced fatigue specimen for CT scan.

Three dimensional reconstruction was also performed with slices using VGStudio MAX software package (Version 1.2.1) for visualization and analysis. The software was used to create image stacks that can be reconstructed into 3D models for better visualization of defect morphology and location. Figure A.5 shows half section and quarter section views as well as a single slice with the largest diameter of each defect.
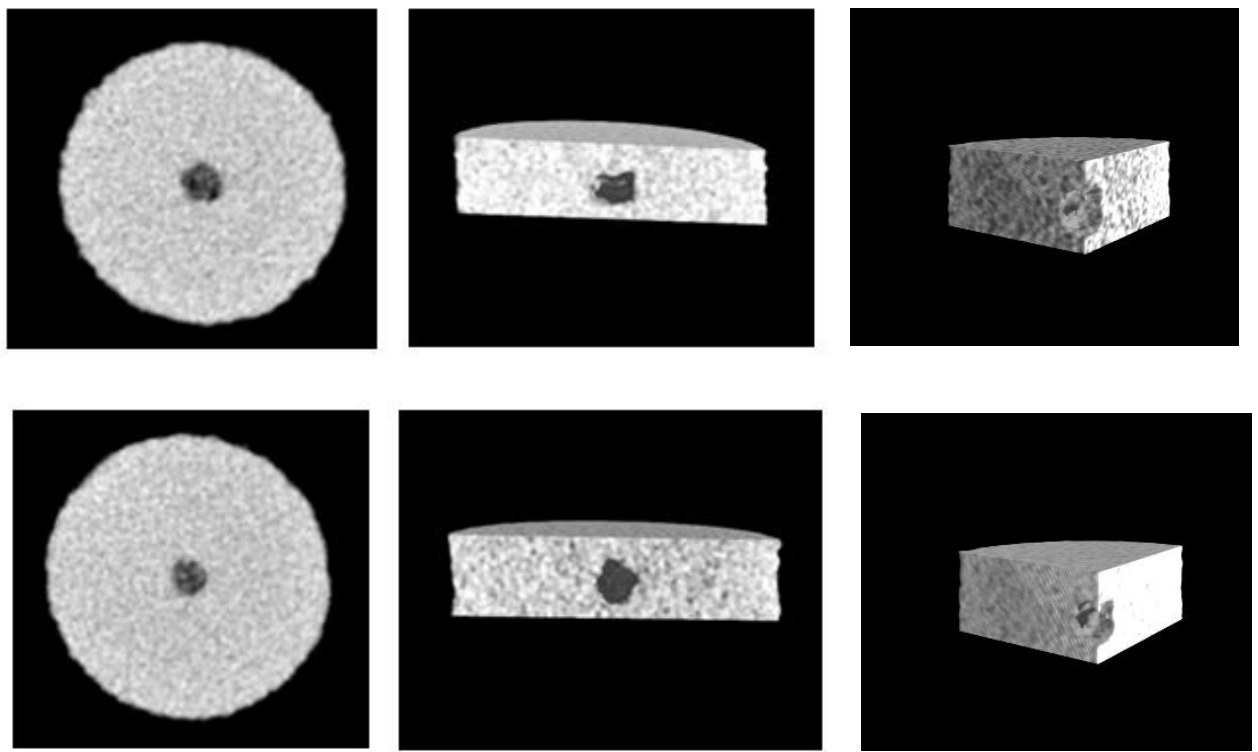

Figure A.5. CAD models and reconstructed inspection results from CT scanning images. 
Since the voxel resolution is known, the dimension of deterministic defect can be determined by counting the number of pixels or slices. For example, the height of the defect is the product of $7 \mu \mathrm{m}$ multiplied by the number of slices in which the defect are visible. And the diameter of the defect can be determined by finding the circumcircle in each slice through an image tool package and measuring its diameter. Based on this, the cylindrical defect, as shown in Figure XX, appears to be no more than $0.378 \mathrm{~mm}$ high, with a diameter of no more than $0.518 \mathrm{~mm}$. The conical defect appears to be no more than $0.406 \mathrm{~mm}$ high with a diameter of no more than $0.483 \mathrm{~mm}$. These results only approximately describe a region that completely contains the defect due to the irregularity of defect contour. But generally the actual defect size is somewhat smaller than CAD models.

\section{A.5 Conclusion}

Destructive Characterization Technique is utilized to verify the formability of deterministic defect. It is found that the defect is hardly formed with a dimension less than $0.21 \mathrm{~mm}$.

Thermography is not a feasible method to detect deterministic defect in the SLM specimens. The heat generation does not occur due to the lack of rubbing surfaces. Ultrasonic testing (A scan) is capable of detecting the deterministic defect and determine the depth by post calculation. It is believed that better results could be achieved if advanced scanning and date processing are performed. CT scan shows the defect by multiple slices. Defect morphology and dimension could be easily acquired. Defect can also be reconstructed to 3D model for the best visualization. Based on the results, micro-CT can be considered as an appropriate way of detecting defects in additive manufactured parts. 


\section{Acknowledgements}

The authors gratefully acknowledge Dr. Stephen Holland of Department of Aerospace Engineering and Center for Nondestructive Evaluation at the Iowa State University, and Dr. Michael Voor and Mr. Seid Waddell of Department of Orthopaedic Surgery at the University of Louisville for their assistance.

\section{Reference}

[1] I. Gibson, D.W. Rosen, B. Stucker. AdditiveManufacturing Technologies: Rapid Prototyping to Direct Digital Manufacturing. Springer, New York, NY, 2009

[2] Jeremy Renshaw, John C. Chen, Stephen D. Holland, et al. The sources of heat generation in vibrothermography. NDT and E International, 44(8), 2011, 736-73 


\begin{abstract}
APPENDIX B
EFFECT OF DEFECTS ON FATIGUE TESTS OF AS-BUILT TI-6AL-4V PARTS

FABRICATED BY SELECTIVE LASER MELTING

Haijun Gong, Khalid Rafi, Thomas Starr, Brent Stucker

This appendix is a paper published in Solid Freeform Fabrication Symposium 2012.

All permissions to using this paper as a part of this dissertation are contained in Appendix
\end{abstract} D.

\begin{abstract}
Defects can be found in parts made using Selective Laser Melting (SLM) due to balling effects and other types of localized irregularities. This study investigates how defects affect the fatigue performance of Ti-6Al-4V samples in an SLM as-built surface finish condition. Fatigue samples were built and heat treated for stress relief. In order to investigate the effect of defects, a series of fatigue samples were designed with built-in cylindrical and double-conical defects. Tests were carried out to correlate maximum stress to the number of cycles to failure. Optical and scanning electron micrographs were utilized to compare and analyze crack initiation and propagation characteristics. Based on the results, the influence of defects on fatigue properties is discussed.
\end{abstract}




\section{B.1 Introduction}

Additive Manufacturing (AM) refers to the process of joining materials to make objects from three-dimensional Computer Aided Design data, usually layer upon layer, as opposed to subtractive manufacturing methodologies, such as traditional machining [1]. It significantly simplifies the process of producing complex $3 \mathrm{D}$ objects directly from CAD data without process planning. The operator only needs some basic dimensional details and a small amount of understanding as to how the AM machine works and the materials that are used [2]. Application of additive manufacturing technology to fabricate complex threedimensional components is one promising direction in industrial and medical field $[3,4]$. This approach is rapidly changing the pattern of designers and processers for creating objects with desired shape and structure. Many additive manufacturing methods have been applied in many variations to satisfy industrial and medical needs for rapidity and flexibility.

Titanium alloys such as Ti-6Al-V4 are widely adopted in aerospace, biomedical and industrial fields due to its inherent properties of fracture resistance, fatigue behavior, corrosion resistance and biocompatibility [5]. In recent years, selective laser melting (a metal powder bed fusion AM process which utilizes a laser to melt metal powder layer-bylayer.) has shown great versatility for fabricating parts from numerous types of metallic powders such as stainless steel, maraging steel, cobalt chromium and Ti-6Al-4V. Titanium alloys are extensively investigated and utilized in SLM processes. Thus, there is interest in quality attributes of SLM Ti-6Al-V4 components as compared to wrought or cast plus post heat treatment-produced components [6-8]. In order to give more insights to designers who are employing this technology, quality and life span of AM components must be carefully 
investigated, especially for metallic parts with defects. Thus, this research focuses on understanding how defects affect the fatigue life of as-built Ti-6Al-4V specimens fabricated using SLM. The stress-life plot is discussed based on the crack pattern of the fracture surfaces. Fractography of each crack pattern is shown for interpreting the crack initiation and propagation process.

\section{B.2 Material and Experimental Plan}

\section{Ti-6Al-4V powder}

The material used for this investigation is EOS Ti64, which fulfills requirements of ASTM F1472 (for Ti-6Al-4V) regarding maximum concentration of impurities [9]. Figure B.1 shows the powder morphology under a scanning electron microscope. The powder was measured using a Microtrac S3000 laser-based particle size analyzer. Its particles have a size distribution between $25 \mu \mathrm{m}\left(\mathrm{D}_{10}\right)$ and $53 \mu \mathrm{m}\left(\mathrm{D}_{90}\right)$. The particle size is nearly normally distributed with Mean Volume Diameter around $38 \mu \mathrm{m}$.

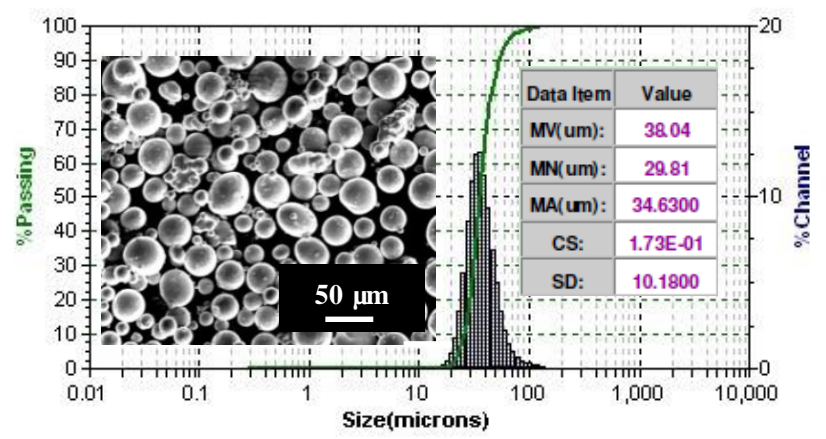

Figure B.1. EOS Ti-6Al-4V powder morphology and particle size distribution.

\section{Fatigue testing}

Fatigue specimens were built vertically along their axis with an as-built gauge surface in an EOS M270 Direct Metal Laser Sintering (DMLS) system. The processing 
parameters from EOS are given in Table B.1. Laser scanning along paralleled paths produced solidified layer. Contour scan was followed for each layer. It is believed that the specimen's density is almost $100 \%$ of STA Ti64 material [7]. Specimens conform to the ASTM E466 standard with a continuous diameter between ends. Some specimens were designed with cylindrical or double-conical (D-conical) internal defect by leaving a void in the CAD file, as shown in Figure B.2.

Table B.1 Process Parameters for Fabricating Ti-6Al-4V Fatigue Specimens

\begin{tabular}{c|c|c|c}
\hline Laser power $(\mathrm{W})$ & Scan speed $(\mathrm{mm} / \mathrm{s})$ & Hatch spacing $(\mu \mathrm{m})$ & Layer thickness $(\mu \mathrm{m})$ \\
\hline 170 & 1250 & 100 & 30 \\
\hline
\end{tabular}

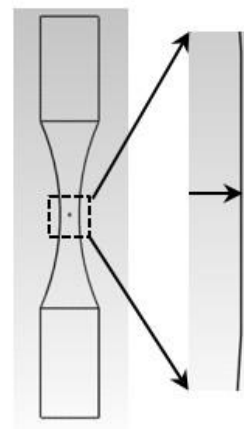

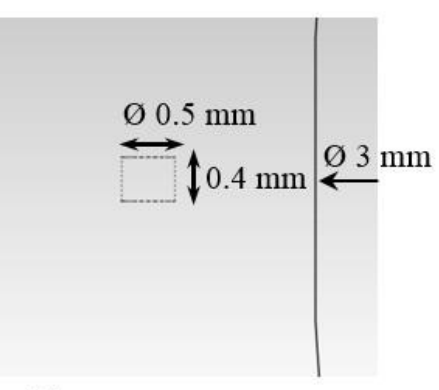

(a)

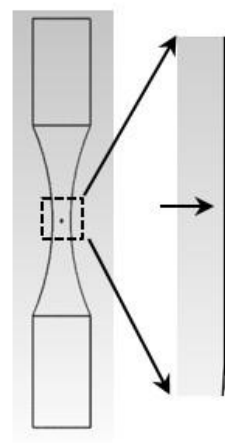

(b)

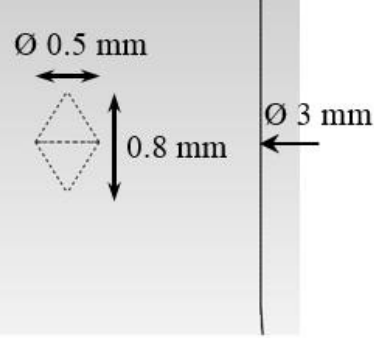

Figure B.2. CAD model of fatigue specimen. (a) Cylindrical defect; (b) D-conical defect.

All specimens were heat treated at 650 Celsius for 4 hours in an argon filled furnace for stress relief and cooled down in the furnace to the room temperature. Light sand blasting was used to clean adhered particles from the surface of the specimens. Fatigue tests were conducted on an Instron ElectroPuls E10000 All-Electric Test Instrument. The specimens were tested using a continuous sinusoidal load with frequency $50 \mathrm{~Hz}$. Stress ratio $R$ (min/max stress amplitudes) is 0.1 . The fracture surfaces were then examined by optical microscopy (Olympus MX51 industrial inspection microscope) and scanning electron 
microscopy (FEI Nova NanoSEM 600) for analyzing the effect of defect on the result of fatigue tests.

\section{B.3 Results and Discussion}

\section{Characteristics of Fatigue Specimens}

An as-built Ti-6Al-4V fatigue specimen is shown in Figure B.2. Through the scanning electron micrograph, it can be seen that the gauge surface is rough and irregular. Adhered particles were removed from the surface of the specimens by sand blasting. But some partially melted particles may be welded to the surface, resulting in convex surfaces which seem like speckles after blasting. But, in general, sand blasting does not affect the feature of as-built surface.
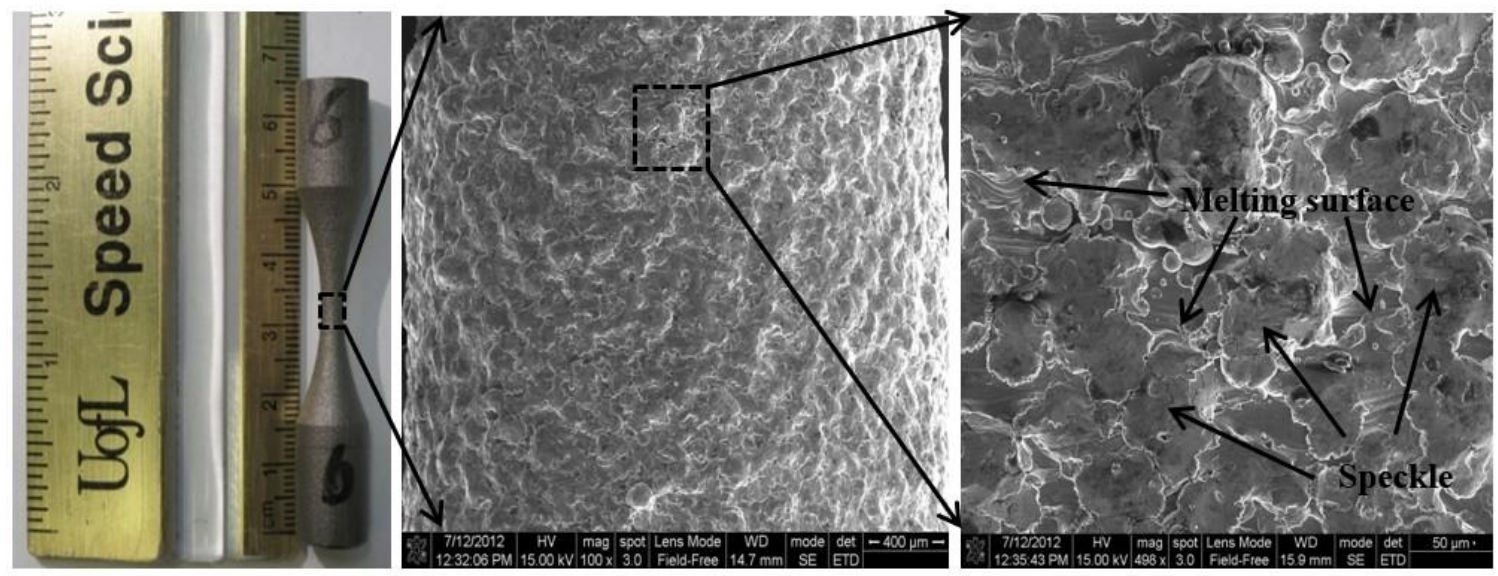

Figure B.3. As-built Ti-6Al-4V fatigue specimen and its surface feature.

\section{Fatigue results}

High cycle fatigue tests can cause cracking in fatigue specimens. Stress-life plots of the fatigue specimens are shown in Figure B.4. The stress life of fatigue specimens without designed defects was rather low at a stress level higher than $500 \mathrm{MPa}$. Three specimens were tested at $500 \mathrm{MPa}$. One of them failed very quickly, while another two did 
not fail at all. When the stress level was lowered to less than $500 \mathrm{MPa}$, even at 10 million stress cycles the specimens did not fail.

It was assumed that the fatigue specimens would have lower stress life if a cylindrical or double-conical defect is included. Therefore, all the specimens with internal defects were tested with a maximum stress no larger than $500 \mathrm{MPa}$ in order to investigate how fatigue life is affected. The results show that the fatigue life at some specific stress level is repeatable, such as fatigue specimens with a cylindrical or double-conical defect at $450 \mathrm{MPa}$. But, at other stress levels, the fatigue life could be distributed across a range. For example, the fatigue life at a $500 \mathrm{MPa}$ stress level is not repeatable, with values between sixty thousand and one million cycles, with no discernible pattern as to cylindrical defects or double-conical defects. Thus it is hard to estimate the number of cycles to failure for the fatigue specimens at this stress level. When lowered to a $400 \mathrm{MPa}$ stress level, the fatigue life of one specimen with a cylindrical defect was even twenty times more than two other specimens. When the maximum stress was lowered to $350 \mathrm{MPa}$, fatigue specimens with defects did not fail until after ten million cycles.

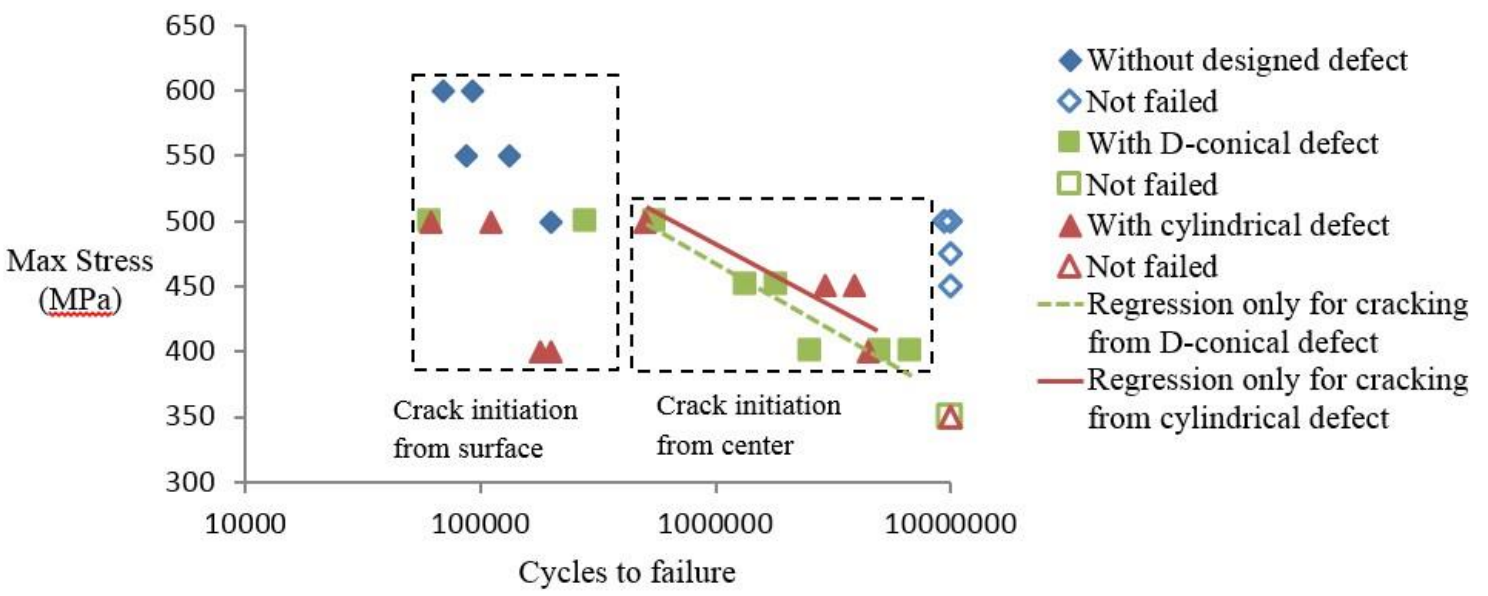

Figure B.4. Stress-life plot for fatigue testing of Ti64 specimens with and without geometric defects. 


\section{Fractography}

Generally the process of fatigue failure is characterized by crack initiation, crack propagation and final failure. Cracks usually initiate at the site with the highest stress concentration. A microscopic view of Ti64 fatigue specimens shows a series of radiating ridges on the fracture surface, which can be traced along the direction to identify the area of the fracture initiation. These radiating ridges occupy most of the fracture surface. In the final fast fracture area, "cup and cone" fracture is formed on the matching surfaces oriented at about $45^{\circ}$ from the tensile axis.

For the fatigue specimens without designed defects, it was found that fatigue crack initiation only occurred at the surface. Fatigue fracture for some specimens occurred outside the gauge section and the fracture extended to the whole cross section and finally failed. Figure B.5 shows a typical fracture surface whose fatigue crack initiated at the gauge surface. A boundary deficiency can be seen at the crack initiation site. The crack propagated from the initiation site over several cycles. Final fracture occurred when the remaining area was too small to carry the maximum load of the final cycle. XRD analysis indicates as-built SLM Ti-6Al-4V contains an HCP phase with both $\alpha$-phase and $\alpha$ ' martensite $[10,11]$. Therefore, the radiating ridges refer to transgranular cleavage of $\alpha$ and $\alpha^{\prime}$ grains. The low failure cycles of these specimens can be attributed to crack growth from some of the $\alpha$ or $\alpha^{\prime}$ grains with very little crack initiation time. Micro striations on grain surfaces can be observed in the crack propagation region at a higher magnification as shown in Figure B.5 (e) and (f). It is assumed that a transition from transgranular cleavage to classic fatigue striations occurred and then changed to final fast fracture in ductile dimple mode [12]. The orientation of these striations varied from grain to grain. Dimpled 
appearance which is characteristic of microvoid coalescence is exhibited in the final fast fracture area. Some regions are located between dimpled areas and appear to be smooth at low magnification.
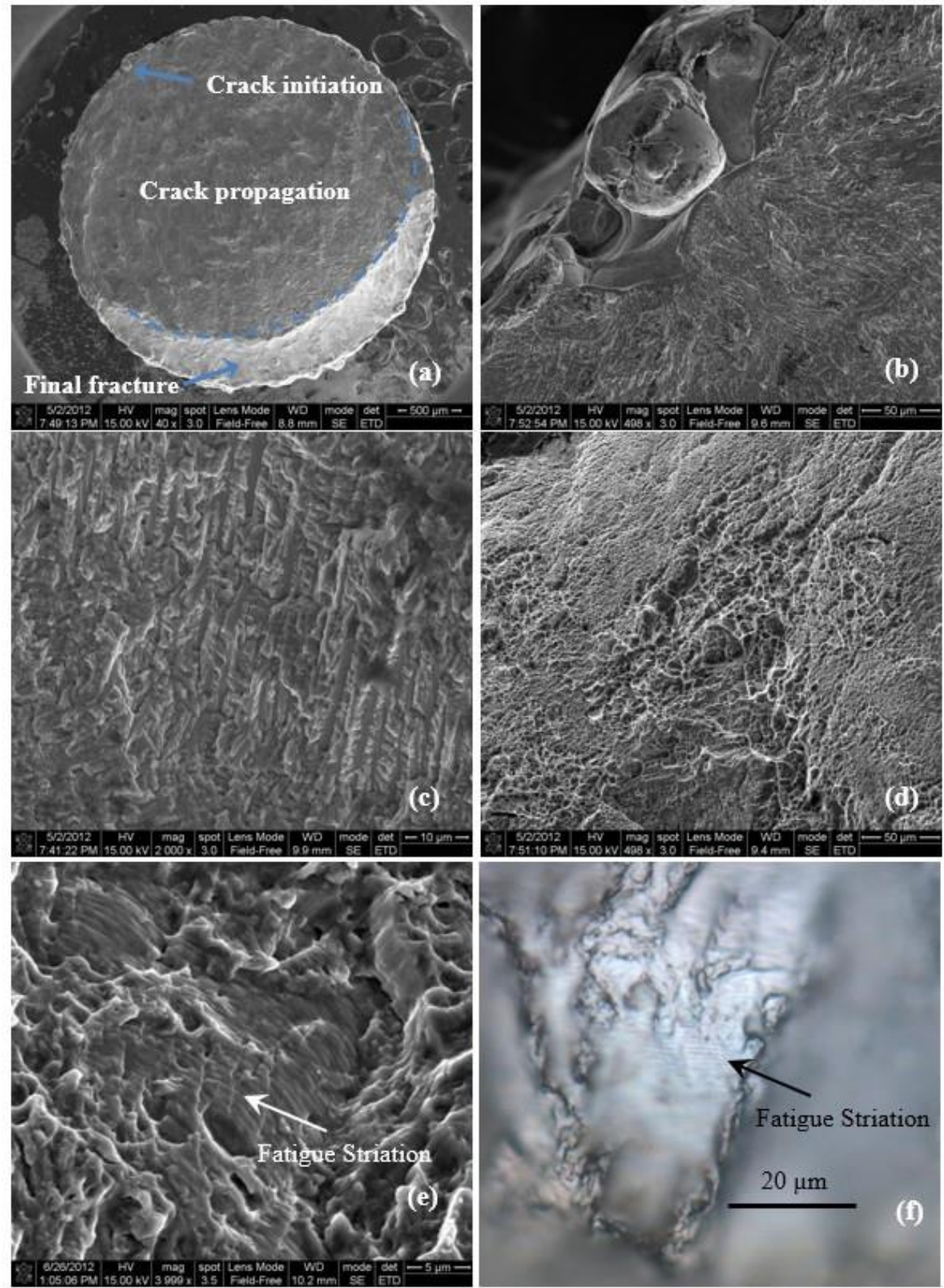

Figure B.5. Fractography of fatigue specimen (crack initiated from gauge surface). (a) Top view; (b) crack initiation site; (c) crack propagation (transgranular fracture); (d) final fast fracture region; (e) fatigue striation in crack propagation region (SEM); (f) fatigue striation (optical microscopy). 
The fracture pattern of fatigue specimens with designed defects is varied, initiating either from the surface of the gauge section or from the designed defect in the interior. Thus, the stress-life plot can be divided into two areas for failed fatigue specimens as shown in Figure B.4. The former fracture pattern has a similar fractography as shown in Figure B.5, accompanied with a lower fatigue life than the latter fracture pattern. This explains why there is such large scatters amongst the fatigue life data for certain stress levels.

When the fatigue crack was initiated from the designed cylindrical defect, the fracture surface is close to an edge of the defect, as shown in Figure B.6. The crack initiated from one of the defect's boundaries. This indicates that geometry-dependent stress concentrations at the edge of a defect can be the cause of crack initiation. However, it is difficult to identify the specific crack initiation site with respect to one of these the $\alpha$ or $\alpha$ ' grain facets. The primary grains in the initiation site may all have orientation pointing along crack propagation. A transgranular cleavage took place in the crack propagation region from and around the crack initiation site, followed by ductile-dimple fast fracture.

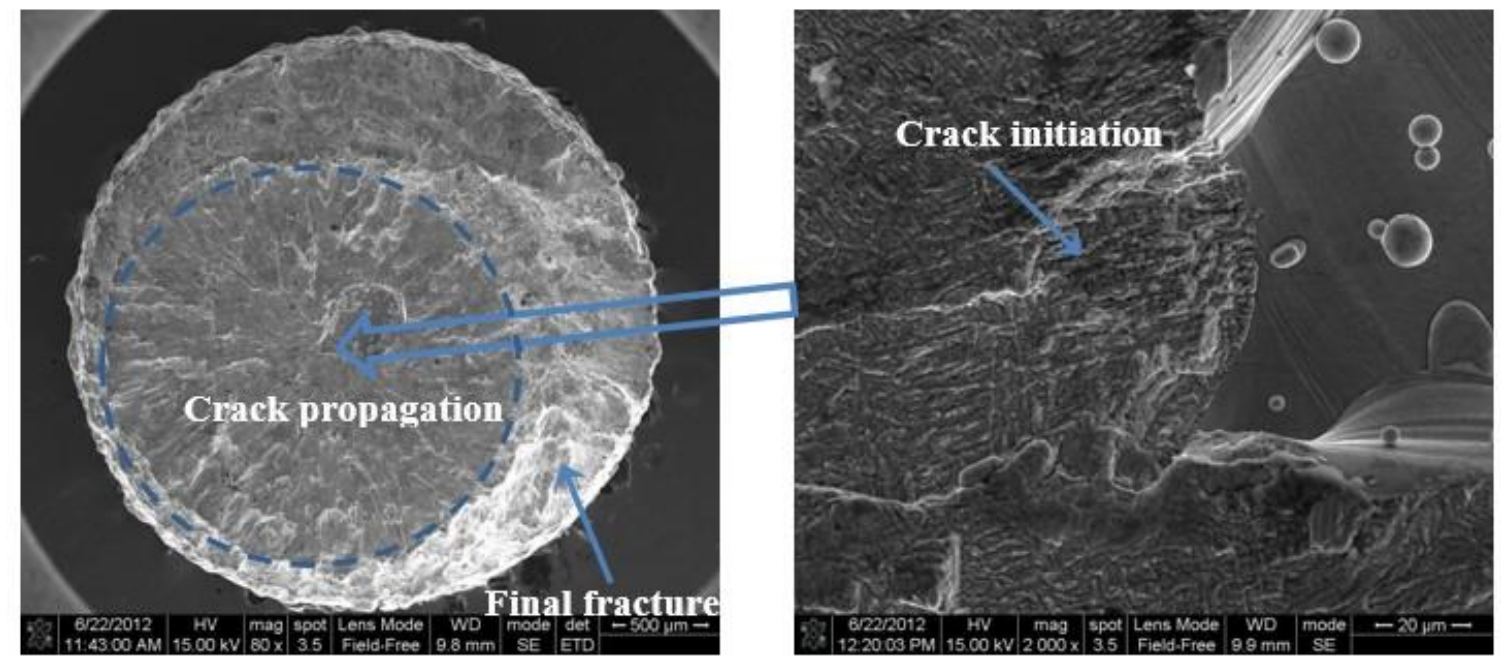

Figure B.6. Fracture surface of fatigue specimen (crack initiated from central cylindrical defect). 
Figure B.7 shows a typical fracture surface with a crack initiated from the doubleconical defect. Transgranular cleavage is the primary crack propagation pattern. Ductile dimple appearance is seen in the fast fracture region.

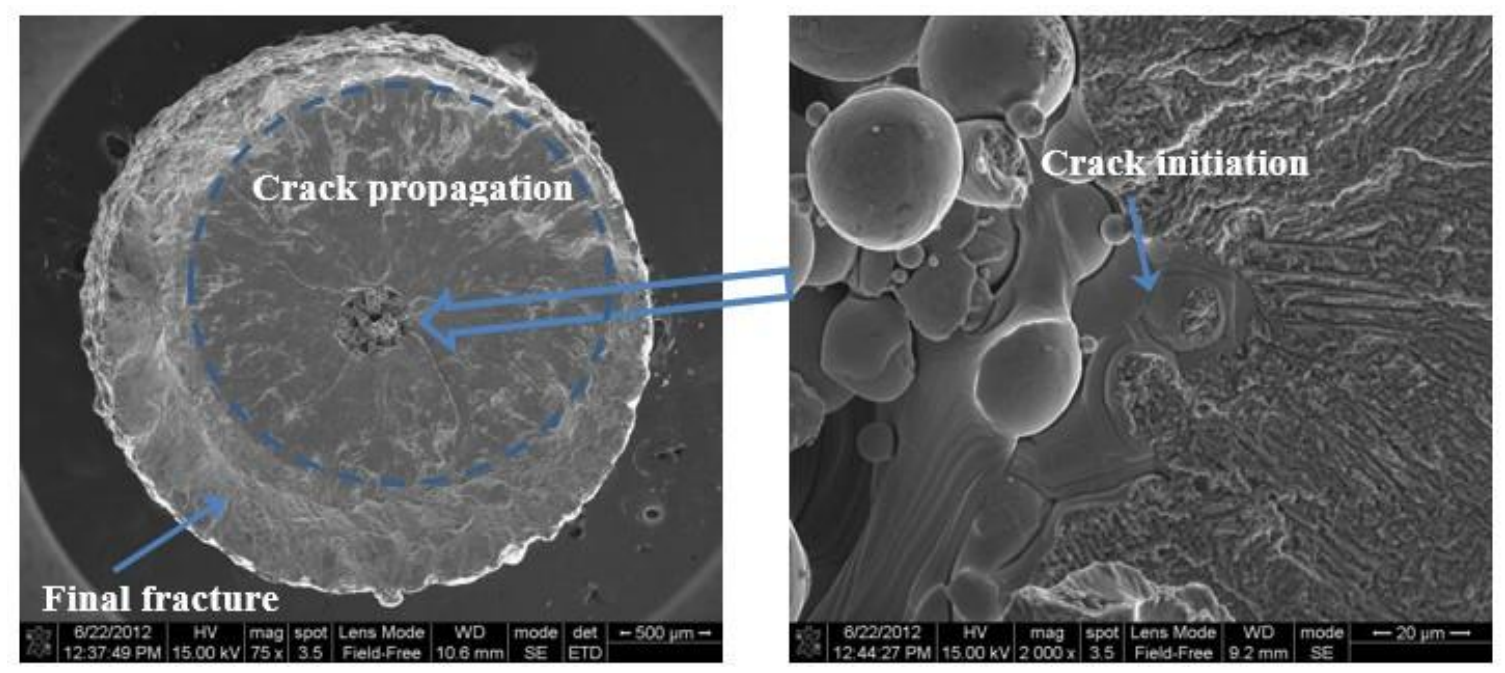

Figure B.7. Fracture surface of fatigue specimen (crack initiated from central doubleconical defect).

According to the fundamentals of fracture mechanics, both cylindrical and doubleconical defects should have a detrimental effect on the fracture strength because the applied stress will be amplified near the defect. The magnitude of this amplification depends on crack orientation and geometry. However, fractography shows no apparent difference in the fracturing process between cylindrical defects and double-conical defects. Moreover, the regression lines show similar tendency between these two types of defects as shown in Figure B.4, if only crack initiations from designed defect are considered in the regression. This indicates that these two defect morphologies do not result in apparent difference of stress amplification with respect to crack initiation.

The rough surface of the gauge section contains a random distribution of defects within the gauge surface. These defects negatively impact the fatigue life as stress risers 
and cause quick failure of Ti-6Al-4V fatigue specimens under the higher stress intensities. This is demonstrated from the stress-life plot in Figure B.4. It was found that as-built fatigue specimens are susceptible to fatigue crack initiation from the surface when the maximum stress is larger than $500 \mathrm{MPa}$. Fatigue specimens with designed defects should encounter the same situation, although no fatigue tests were conducted at stress levels above $500 \mathrm{MPa}$ for those specimens. At stresses below 500MPa, as-built surface defects have no apparent impact on fatigue life. This could be the result of microstructural characteristics around surface defects compared to those near designed internal defects. When Ti-6Al-4V fatigue specimens are free from any internal/surface defects or the defects are not sufficient enough to act as stress raisers then the fatigue properties are strongly influenced by the morphology and arrangement of the $\alpha$ and $\beta$ phases. SLM fabricated Ti6Al-4V exhibits a lamellar microstructure. In lamellar microstructures fatigue cracks initiate at slips bands within $\alpha\left(\alpha^{\prime}\right)$ lamellae or at $\alpha\left(\alpha^{\prime}\right)$ along prior $\beta$ grain boundaries [13]. This can result in crack initiation to occur from the interior. Thus, in addition to the role of defects themselves, further investigations are merited to better understand the microstructural differences between skin and core areas of SLM-produced Ti-6Al-4V specimens to help understand how these might affect crack initiation.

\section{B.4 Conclusion}

Defects play a critical role in SLM Ti-6Al-4V fatigue specimen performance. Asbuilt surfaces become crack initiation sites, especially when the stress intensity is higher than $500 \mathrm{MPa}$. Designed internal defects also affect the fatigue life of SLM Ti-6Al-4V materials so that the fatigue limit is lowered during fatigue testing. In these tests, no 
significant differences between cylindrical and double-conical defects were observed. In order to explain the effect of defects on Ti-6Al-4V fatigue life, further studies concerning the microstructure and morphology of the surface of fatigue specimens are required.

Acknowledge

The authors gratefully acknowledge the support of the Office of Naval Research, awards N00014-09-1-0147 and N00014-10-1-0800, Technical Monitor: Dr. Ignacio Perez. The authors also express their gratitude to the staff of the Rapid Prototyping Center at the University of Louisville for their assistance.

Reference

[1] American Society for Testing and Materials (ASTM). Standard Terminology for Additive Manufacturing Technologies (F2792-12). http://www.astm.org.

[2] I. Gibson, D.W. Rosen, B. Stucker. AdditiveManufacturing Technologies: Rapid Prototyping to DirectDigital Manufacturing. Springer, New York, NY, 2009

[3] G. Levy, R. Schindel, J.P. Kruth. Rapid Manufacturing and Rapid Tooling with Layer Manufacturing (LM) Technologies, State of the Art and Future Perspectives. CIRP AnnalsManufacturing Technology, 52(2), 2003, 589-609

[4] B. Vandenbroucke, J.P. Kruth. Selective Laser Melting of Biocompatible Metals for Rapid Manufacturing of Medical Parts. Rapid Prototyping Journal, 13(4), 2007, 196-203

[5] W.O. Soboyejo, T.S. Srivatsan. Advanced Structural Materials: Properties, Design Optimization, and Applications. CRC Press 2006, 359-400

[6] S. Kumar. Microstructure and Wear of SLM Materials. Solid Freeform Fabrication Symposium, 2008, 128-142

[7] M.K.E. Ramosoeu, G. Booysen, T.N. Ngonda, et al. Mechanical Properties of Direct Laser Sintered Ti-6Al-V4. Materials Science and Technology Conference (MS\&T), 2011, 1460-1468 
[8] B. Van Hooreweder, D. Moens, R. Boonen, et al. Analysis of Fracture Toughness and Crack Propagation of Ti-6Al-4V Produced by Selective Laser Melting. Advanced Engineering Materials, 14(1-2), 2012, 92-97

[9] EOS Titanium Ti64 for Aerospace and Engineering Applications and Biomedical Implants. EOS GmbH (www.eos.info)

[10] L. Facchini, E. Magalini, P. Robotti, et al. Ductility of a Ti-6Al-4V Alloy Produced by Selective Laser Melting of Prealloyed Powders. Rapid Prototyping Journal, 16(6), 2010, $450-459$

[11] L.E. Murr, S.M. Gaytan, D.A. Ramirez, et al. Metal Fabrication by Additive Manufacturing Using Laser and Electron Beam Melting Technologies. J. Mater. Sci. Technol., 28(1), 2012, 1-14

[12] W.A. Glaeser, B.H. Lawless. Behavior of Alloy Ti-6Al-4V under Pre-Fretting and Subsequent Fatigue Conditions. Wear, 250, 2001, 621-630

[13] T. Mohandas, D. Banerjee, V.V Kutumba Rao. Fusion Zone Microstructure and Porosity in Electron Beam Welds of an $\alpha+\beta$ Titanium Alloy. Metal. Trans. 30A, 1998, 789798 


\title{
APPENDIX C \\ MICROSTRUCTURES AND MECHANICAL PROPERTIES OF TI6AL4V PARTS \\ FABRICATED BY SELECTIVE LASER MELTING AND ELECTRON BEAM \\ MELTING
}

H. Khalid Rafi, N.V. Karthik, Haijun Gong, Thomas L. Starr, Brent E. Stucker

This appendix was accepted and will be published by Journal of Materials Engineering and Performance. All permissions to using this paper as a part of this dissertation are contained in Appendix D.

\begin{abstract}
This work compares two metal additive manufacturing processes, Selective Laser Melting (SLM) and Electron Beam Melting (EBM), based on microstructural and mechanical property evaluation of Ti6Al4V parts produced by these two processes. Tensile and fatigue bars conforming to ASTM standards were fabricated using Ti6Al4V ELI grade material. Microstructural evolution was studied using optical and scanning electron microscopy. Tensile and fatigue tests were carried out to understand mechanical properties and to correlate them with the corresponding microstructure. The results show differences in microstructural evolution between SLM and EBM processed Ti6Al4V and their influence on mechanical properties. The microstructure of SLM processed parts were composed of an $\alpha^{\prime}$ martensitic phase whereas the EBM processed parts contain primarily $\alpha$ and a small amount of $\beta$ phase. Consequently, there are differences in tensile and fatigue
\end{abstract}


properties between SLM and EBM produced Ti6A14V parts. The differences are related to the cooling rates experienced as a consequence of the processing conditions associated with SLM and EBM processes.

Keywords: SLM, EBM, Tensile testing; Fatigue testing; Microstructure.

\section{C.1 Introduction}

Selective laser melting (SLM) and electron beam melting (EBM) are two powder bed fusion-based additive manufacturing processes used to fabricate metallic parts $[1,2]$. These processes are of interest due to several advantages over conventional manufacturing methods. Freedom to fabricate intricate geometries, optimum material usage, elimination of expensive tooling etc. are some of the notable advantages of additive manufacturing processes. In these processes the CAD model of the part is fed to the machine where preprocessing software slices the model into layers of finite thickness. A powder layer is deposited on to a base plate above the build platform. A focused laser/electron beam scans the powder bed-based on the sliced CAD data. The scanning results in localized melting and solidification of the powder to form a layer of the part. Subsequent layers are built one over the other by lowering the build platform equivalent to the layer thickness until the part is completed.

SLM utilizes a fiber laser heat source. The four main parameters in SLM are laser power, scan speed, hatch spacing and layer thickness. Generally the process is characterized by high scan speeds and high thermal gradients, leading to high cooling rates. High cooling rates result in non-equilibrium microstructures which may require heat 
treatment for certain applications. The SLM build chamber is continuously flushed with inert gas to reduce oxygen level. Typical layer thickness lies in the range of $20 \mu \mathrm{m}$ to 100 $\mu \mathrm{m}$. SLM is capable of processing standard materials like Ti6Al4V, 316L, 17-4PH, 155PH, hot work steels, Cobalt-based and Nickel-based alloys [3] and more. A description of SLM processes has been detailed elsewhere [4].

Arcam EBM technology uses an electron beam to melt powder layer. Electron beam-powder interactions are substantially different than laser-powder interactions. The penetration depth of an electron beam into the irradiated material is multiple times greater than it is with a laser beam [5]. When the high speed electron beam interacts with the powder layer, kinetic energy is converted into thermal energy, causing the powder to melt. The build chamber is kept at an elevated temperature (approx. $700{ }^{\circ} \mathrm{C}$ ) in a vacuum environment. Elevated temperatures help minimize thermally induced residual stresses and the formation of non-equilibrium microstructures. The high intensity electron beam first preheats the powder at a very high scan speed, large focal spot and low beam current. Preheating of the powder can help lower moisture content and thus reduce the possibility of oxygen pickup. More importantly preheating can reduce residual stress buildup by bringing down the temperature gradient between successive layers during processing. The preheating stage is followed by a melting stage where the electron beam scans the powder at a lower scan speed, smaller spot size and higher beam current. Once the build is completed the part is allowed to cool slowly from $700{ }^{\circ} \mathrm{C}$ to room temperature. Due to the higher beam intensities and scan available with electron beams, the EBM process is much faster than the SLM process. A description of EBM processes has been detailed elsewhere [6]. 
Previous studies carried out by different researchers showed typical microstructures and related properties for SLM and EBM produced materials. Thijs et al studied [7] the influence of process parameters and the scanning strategy on the microstructural evolution during SLM processing of Ti64. They observed the resulting microstructure as acicular martensite as a consequence of very high cooling rates. The microstructure was significantly affected by factors such as high localized heat inputs, very short interaction times, local heat transfer conditions, and processing conditions like scanning velocity, hatch spacing (the distance between two adjacent scan vectors) and scanning strategy. Facchini et al also found SLM produced Ti64 microstructures to be as completely martensitic. Song et al [8] studied the effect of process parameters in terms of microstructure, densification, surface roughness and microhardness for Ti64. They suggested a laser power of $110 \mathrm{~W}$ and scan speed of $0.4 \mathrm{~m} / \mathrm{s}$ in a continuous melting mode to obtain a Ti64 part with maximum density. Other than the microstructural aspects, previous studies performed by Yadroitsev et al.[9], Morgan et al.[10], and Yasa et al. [11] have provided the details on the influence of substrate, energy input, laser pulsing and laser irradiation parameters on process stability and dimensional accuracy of the final product.

Murr et al carried out characterization and comparison of Ti64 produced by EBM processing with wrought products. Microstructural characterization revealed acicular $\alpha$ and associated $\beta$ microstructure [6]. Prior $\beta$ grains form epitaxially and extend through many layers which is a direct consequence of the thermal gradient in the build direction [12]. Facchini et al also showed a very fine and acicular morphology when Ti64 parts were produced using EBM [13]. 
Although the microstructural aspects of SLM produced and EBM produced samples have been studied, little attention has been paid to a comparison and contrast between these processes with respect to a given material. Therefore, this work is aimed at comparing SLM and EBM processes in terms of microstructure, tensile properties and fatigue properties of Ti64.

\section{C.2 Experimental Methods}

Ti64 parts were produced using an EOS M270 SLM machine and an Arcam S400 EBM machine. Ti64 powder was procured from each respective machine manufacturer. Powder particle size was measured using a ‘Microtarc 3000' particle analyzer. The average particle size of the powder supplied by EOS was $36 \mu \mathrm{m}$ and the powder supplied by Arcam was $60 \mu \mathrm{m}$. The particle size distribution and corresponding SEM-SE images of Arcam Ti64 powder and EOS Ti64 powder are shown in Figure C.1. Cylindrical specimens and specimens conforming to ASTM standards (ASTM: E8) for tensile testing and for fatigue testing (ASTM: E466) were fabricated. The as-built cylindrical specimens were analyzed for surface finish and sectioned for metallographic characterization. Metallographic specimens were prepared following standard specimen preparation methods. Optical microscopy (OM) and Scanning Electron Microscopy (SEM) were used for microstructural characterization. SEM-EDS (energy dispersive spectroscopy) was carried out to compare any compositional differences which may have occurred due to differences in processes characteristics. OM was carried out on an Olympus optical microscope and SEM was carried out in FEI FEG-SEM. X-ray diffraction (XRD) was carried out to analyze the differences in phase composition. Tensile tests at room temperature were performed for 
samples built in both vertical and horizontal orientations using an Instron $50 \mathrm{kN}$ tensile testing machine. Rockwell hardness testing was carried out using a Wilson Rockwell Hardness 3JR tester. High cycle fatigue tests at room temperature were performed on a $10 \mathrm{kN}$ Instron Electropulse 10000 fatigue testing machine. Fatigue tests were performed at a stress ratio of $\mathrm{R}=0.1$ and a sinusoidal frequency of $50 \mathrm{~Hz}$. Fatigue tests were stopped when specimens broke or the fatigue cycles reached $10^{7}$ cycles.
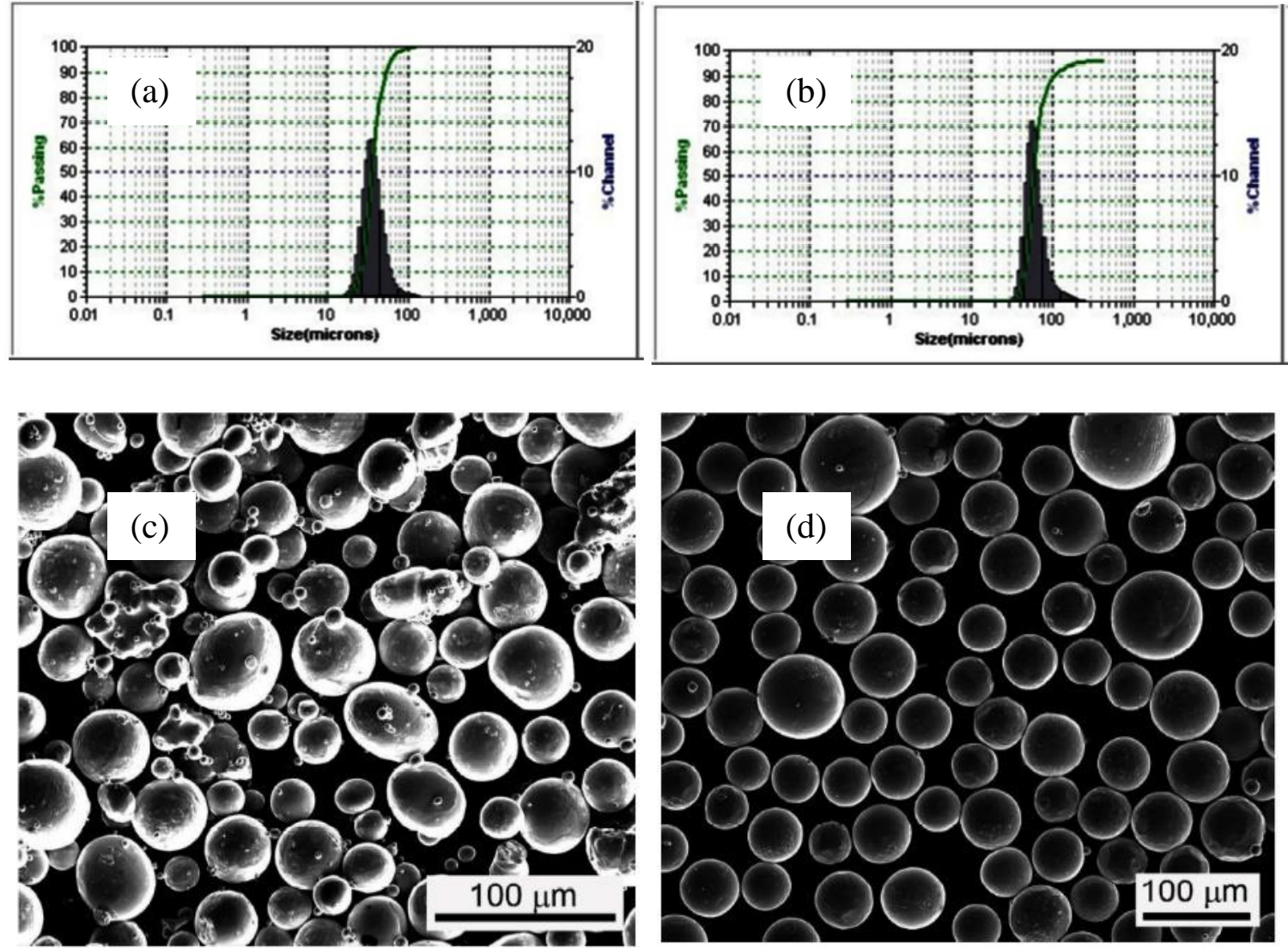

Figure C.1. (a) Powder size distribution of EOS supplied Ti64 powder (Avg particle size: $36 \mu \mathrm{m}$ ); (b) powder size distribution of Arcam supplied Ti64 powder (Avg particle size: $60 \mu \mathrm{m}$ ); (c) SEM-SE image of EOS supplied Ti64 powder; (d) SEM-SE image of Arcam supplied Ti64 powder.

\section{C.3. Results and Discussions \\ C.3.1 Surface characteristics}


The external surfaces of the parts fabricated by SLM and EBM processes have different surface roughness because of the difference in scan speed, powder particle size and layer thickness. Figure C.2a and C.2b shows as-built tensile samples produced by SLM and EBM respectively. From the external appearance it is clear that there is a difference in the surface condition. Figure C.3 shows magnified SEM images of the external surfaces of solid cylindrical specimens fabricated by SLM and EBM. The surfaces of parts fabricated by SLM are relatively smooth when compared to EBM fabricated parts. Figure C.3a-3b show the external surfaces of vertical and horizontal SLM samples respectively. The surfaces of vertically built samples are characterized by a wavy appearance without any discontinuity. For horizontally built cylindrical samples the curved surfaces are formed by consecutive steps. Figure C.3c and C.3d shows the external surface of EBM produced samples in vertical and horizontal orientations respectively. Partly melted powders sticking to the surface and gaps showing distinct layers are visible at the external surface of vertically built samples. Similar to SLM samples, the horizontally built EBM sample surfaces also showed overlapping layers forming steps on the curved external surface.
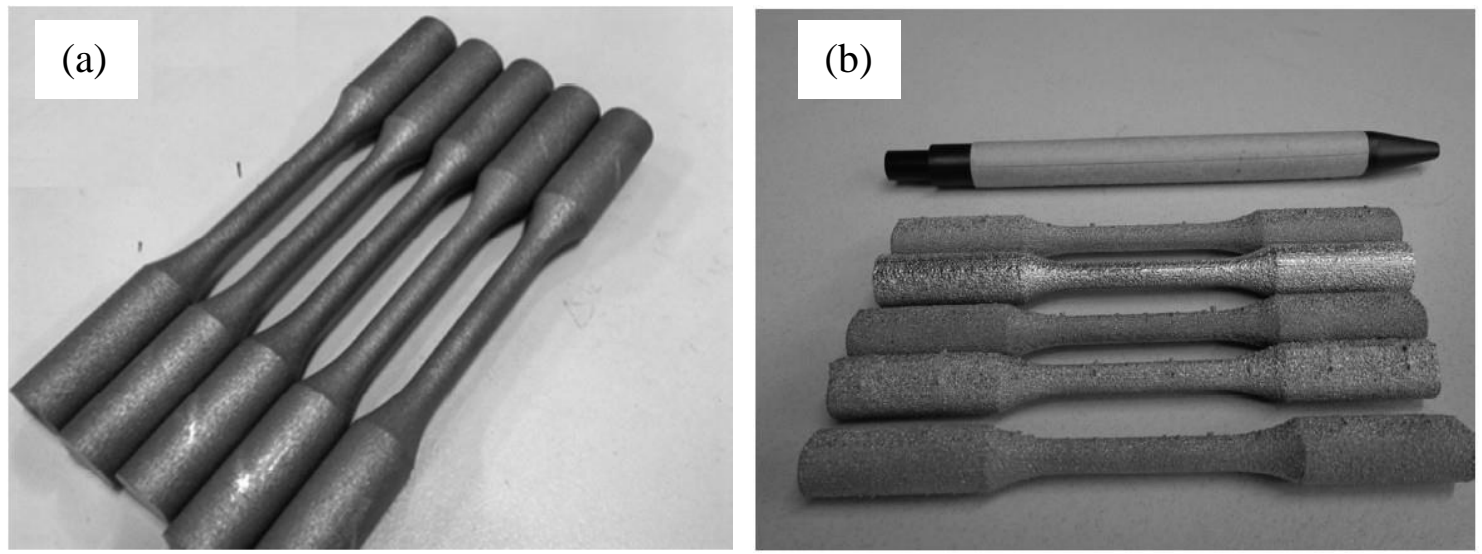

Figure C.2. a) As-built tensile samples produced in SLM; b) as-built tensile samples produced in EBM. 


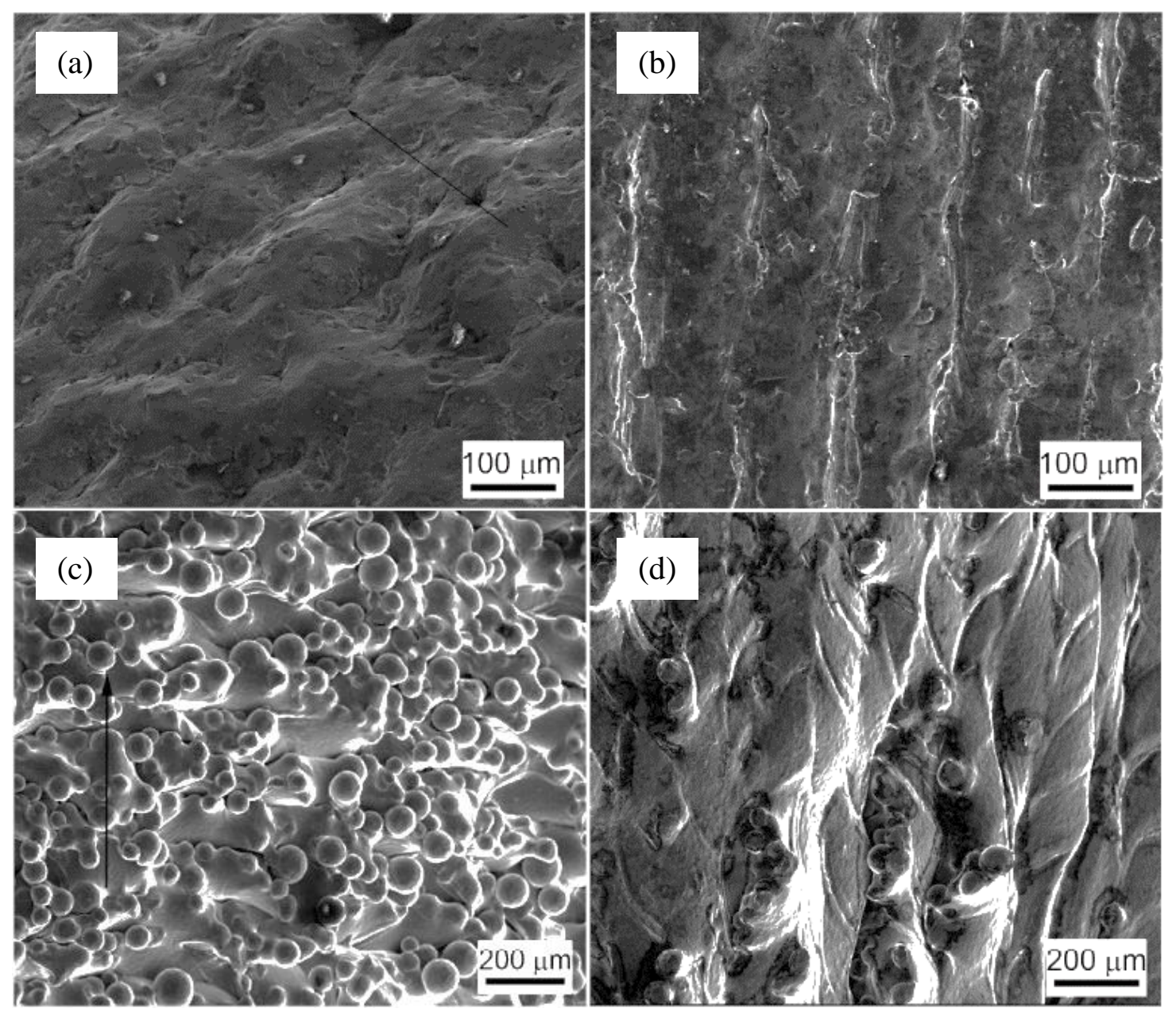

Figure C.3. a) External surface of a vertically built SLM sample (arrow shows the build direction); b) external surface of a horizontally built SLM sample (build direction is perpendicular to the image plane); c) external surface of a vertically built EBM sample (arrow shows the build direction); d) external surface of a horizontally built EBM sample (build direction is perpendicular to the image plane).

The smooth surfaces for SLM fabricated parts are present due to the thinner layers, slower scan speeds, and finer powder particle sizes. The scan speed in EBM is an order of magnitude higher when compared to the scan speed in SLM. This makes the EBM process faster at the expense of poor surface finish. Formation of relatively thicker layers $(70 \mu \mathrm{m}$ as compared to $30 \mu \mathrm{m}$ in SLM process) in the EBM process cause a more pronounced 'stairstep effect' which results in a greater surface roughness. Surface conditions can have 
a significant effect on mechanical properties, particularly for fatigue. For many applications the parts must be subjected to machining to obtain a desired surface finish.

\section{C.3.2 Microstructure}

Microstructural evolution is primarily a function of cooling rate. The materials processed in SLM and EBM undergo very high cooling rates. Figure C.4 shows the optical microstructure of SLM processed Ti64. SLM processing of Ti64 resulted in a complete martensitic (́́) microstructure as expected [7]. Martensitic laths originated from the prior $\beta$ grain boundaries and fill the columnar grains. The morphology of lath martensite can be observed from the SEM-SE image shown in Figure C.5. The martensitic lath width is about $1-2 \mu \mathrm{m}$ and the length is close to the width of the columnar grains.

The optical micrographs of EBM produced Ti64 given in Figure C.6 show a completely different microstructure. The microstructure is mainly composed of an $\alpha$ phase and a small amount of $\beta$ within the prior $\beta$ columnar grains oriented along the build direction. The $\alpha$ phase posses a lamellar morphology with $\beta$ surrounding the $\alpha$ lamellae boundary. The $\alpha$ lamellae are arranged in a Widmanstatten/basket weave structure with different sizes and orientations, and forms alpha platelet colonies within the columnar grains as can be seen in Figure C.7. This means that the SLM and EBM processes produce different microstructures at least for Ti64. However, in both cases prior $\beta$ columnar grain boundaries are clearly visible. This implies that the primary mode of solidification still remains $\beta$, which is characteristic of Ti64 alloys irrespective of the process. Therefore the difference in microstructure is because of the differences in cooling rate when the $\beta$ 
transforms to $\alpha$ as it cools through the transus temperature. Since the SLM process has cooling rates on the order of $10^{6} \mathrm{~K} / \mathrm{s}$, this results in the transformation of $\alpha$ to $\alpha$.

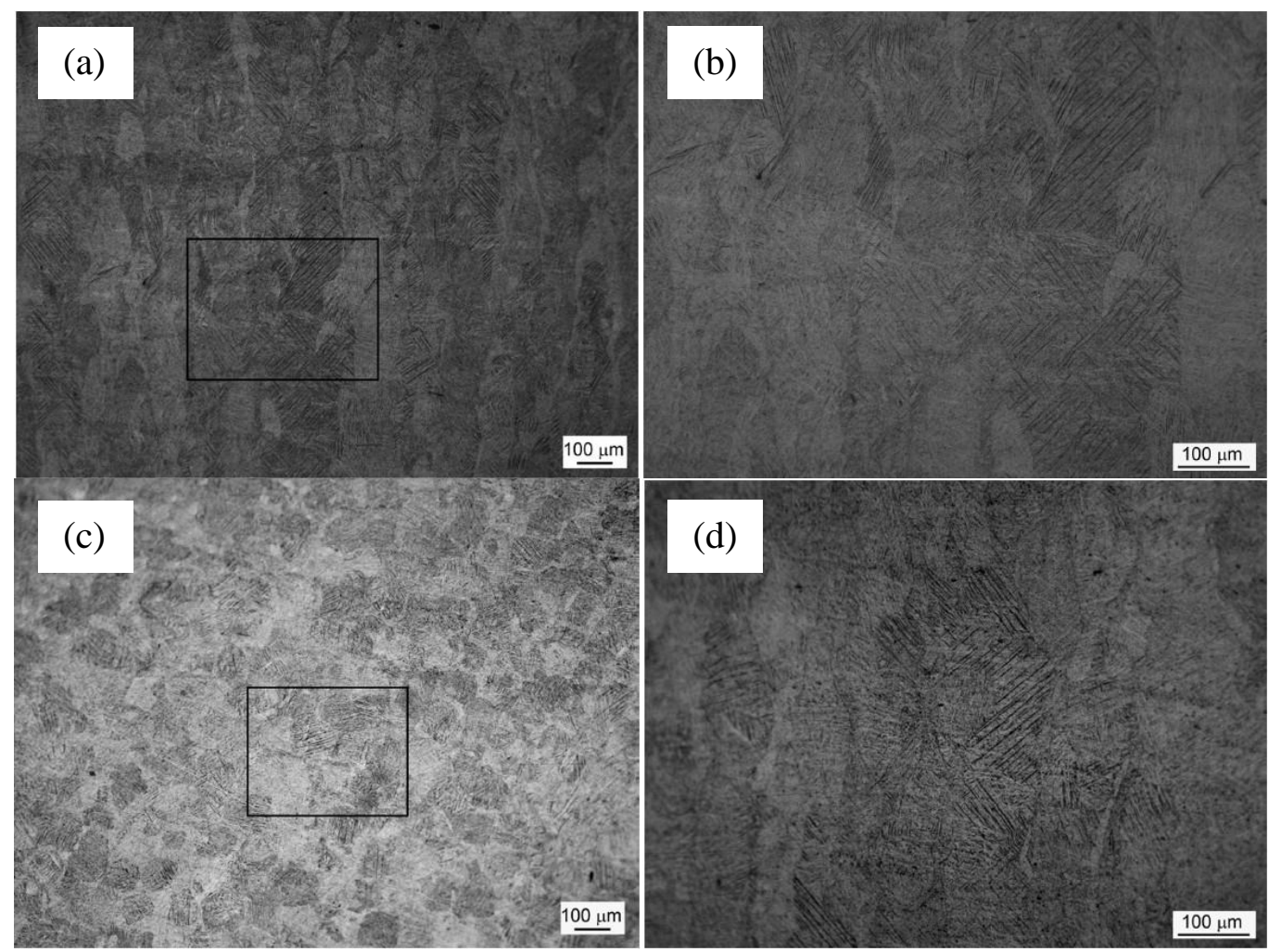

Figure C.4. Optical micrographs of SLM produced Ti64 samples. a) Longitudinal crosssection showing columnar grains; b) high magnification longitudinal cross-section image showing fine $\alpha$ martensitic laths (from the boxed region in 'a'); c) transverse cross-section showing bundles of columnar grains; $d$ ) high magnification transverse cross-section image showing fine $\alpha$ martensitic laths in a columnar grain (from the boxed region in 'c').

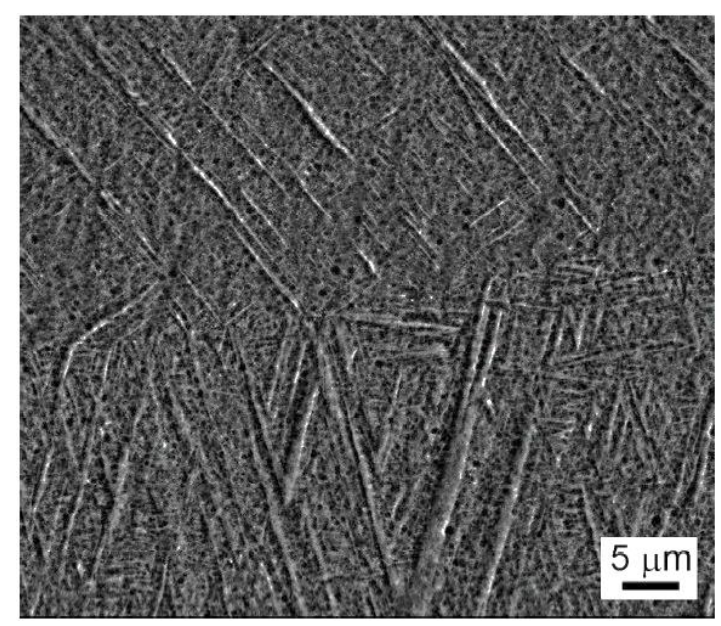

Figure C.5. SEM-SE image of SLM produced Ti64 sample. 


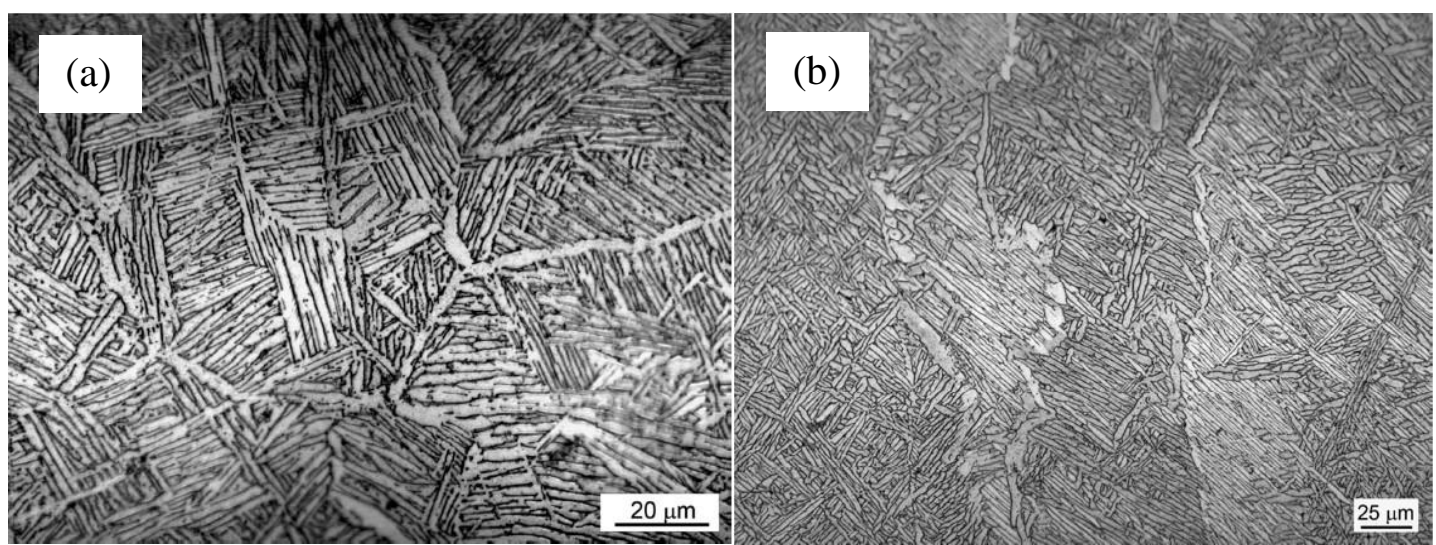

Figure C.6. Optical micrograph of EBM produced Ti64 samples. a) Transverse crosssection; (b) longitudinal cross-section.

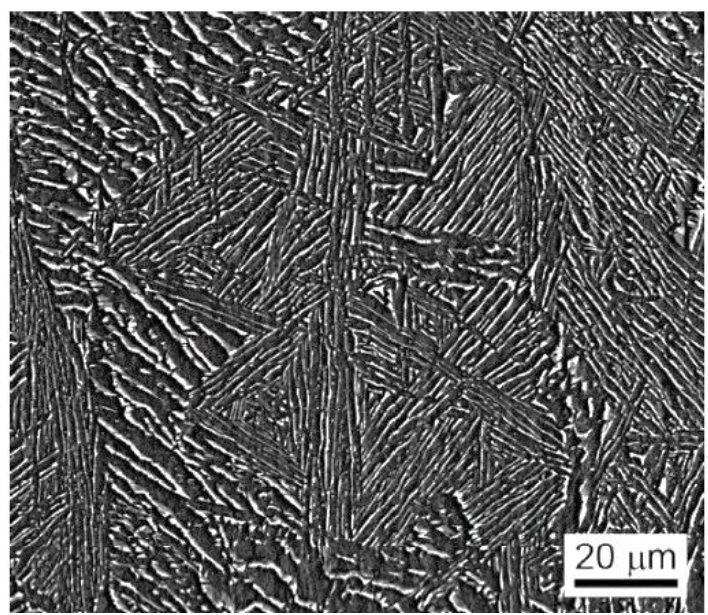

Figure C.7. SEM-SE image showing Widmanstatten structure in EBM produced Ti64 sample.

For EBM the build chamber is maintained at a temperature of $650-700{ }^{\circ} \mathrm{C}$ which is well above the Ms temperature for Ti64. Therefore, even though the cooling rates are higher at elevated temperatures, the material cools down to an isothermal temperature of $650-700{ }^{\circ} \mathrm{C}$. This does not allow the transformation of $\alpha$ to $\alpha$. After completion of the build, the slow cooling rates from $700{ }^{\circ} \mathrm{C}$ to room temperature within the build chamber result in the formation of $\alpha$ platelets. This means that there would be a corresponding difference in mechanical properties between SLM processed and EBM processed samples. 
The microstructures of SLM and EBM produced Ti64 also differ from the microstructure of conventional wrought materials as shown in Figure C.8. The wrought Ti64 microstructure is composed of both $\alpha$ and $\beta$ grains oriented in the rolling direction. The suitability of the microstructure obtained from SLM and EBM processes for different applications are still a matter of debate. Since Ti64 and many other alloys respond well to different heat treatments, selecting an appropriate post-heat treatment method can likely give the desired properties [14].

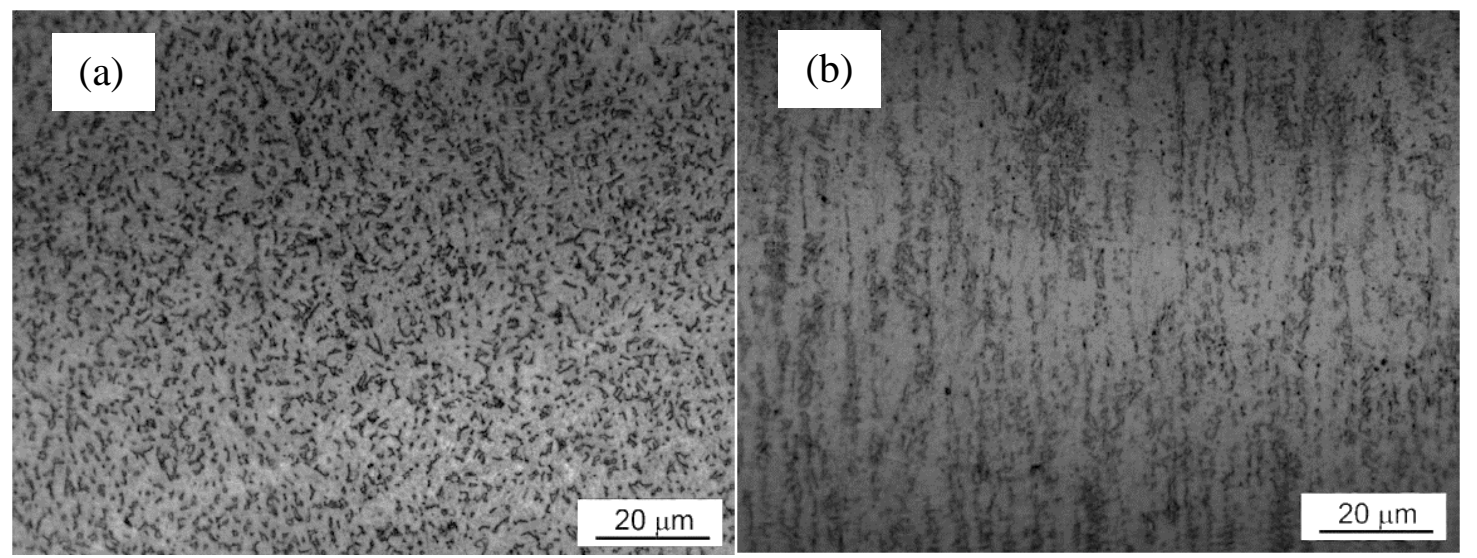

Figure C.8. Optical micrograph of wrought Ti64 (annealed and rolled). a) Transverse crosssection; b) longitudinal cross-section.

\section{C.3.3 Composition and phase analysis}

The chemical composition of SLM and EBM produced samples were compared using SEM-EDS. Though SEM-EDS is a semi quantitative method which lacks accuracy for obtaining the exact chemical composition, it is quite good for comparative purpose. Figure C.9a and C.9b show the EDS spectrum and the composition in wt\% for SLM Ti64 and EBM Ti64, respectively. No significant difference is observed in the chemical composition, indicating that the formation of martensite in SLM Ti64 is not influenced by a variation in alloying element composition. 

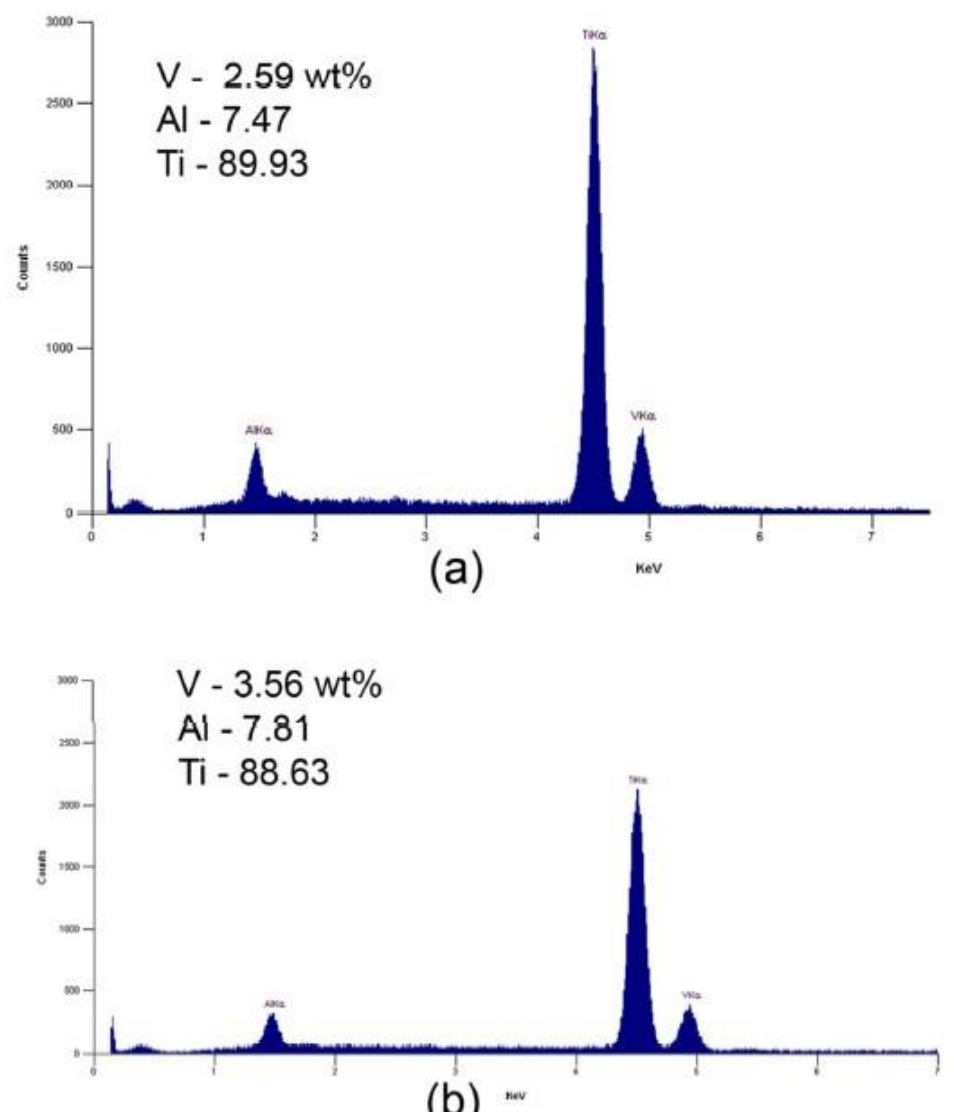

Figure C.9. EDS spectrum and the composition in wt\% of Ti64 samples produced by a) SLM and b) EBM.

Figure C.10a and C.10b show the XRD spectrum of SLM processed Ti64 and EBM processed Ti64, respectively. Analysis of XRD patterns from the SLM and EBM processed Ti64 show similar diffraction patterns. All the peaks can be identified as $\alpha / \alpha ́$. As $\alpha$ and $\alpha$ have the same crystal structure, hcp, it is difficult to differentiate the peaks though they are two different phases. However, the peak intensities in SLM processed Ti64 are slightly lower compared to the spectra of EBM processed Ti64. This is because of the finer structure in SLM processed Ti64. 

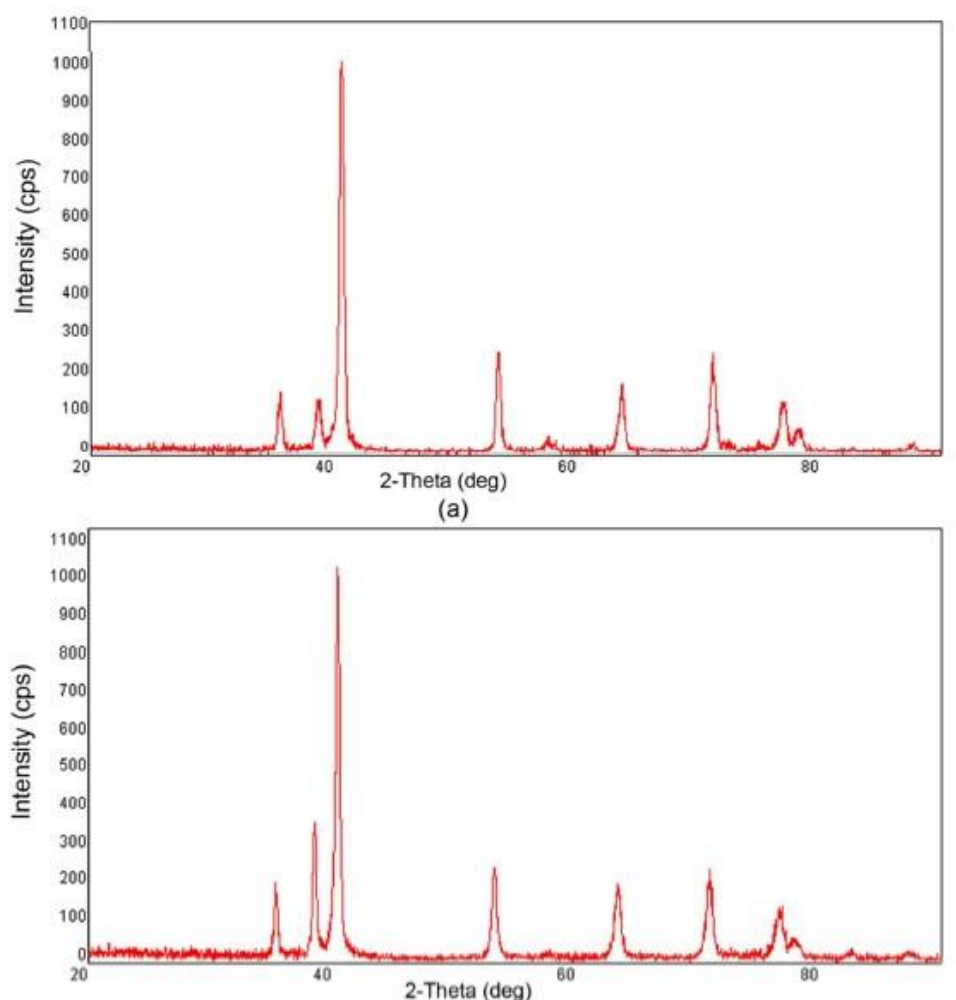

(b)

Figure C.10. XRD spectrum of Ti64 samples produced by a) SLM and b) EBM.

\section{C.3.4 Tensile properties}

Tensile results of EBM and SLM produced Ti64 samples are summarized in Table C.1. Corresponding stress-strain curves are shown in Figure C.11. The results are the mean values based on five duplicate tests. The yield strength, ultimate tensile strength, and percentage strain were obtained as direct output from the tensile testing machine. The percentage strain-to-failure was measured using a clip-on extensometer that was attached to the gage section of the test specimen. Substantial difference in tensile properties can be seen between the EBM produced Ti64 and the SLM produced Ti64 samples. However, the tensile strength values are comparable with or even better than the standard Ti64 material data given in the ASM Handbook [15]. The tensile results are due to the differences observed in the microstructures. The higher tensile strength observed in SLM produced 
Ti64 can be attributed to the martensitic microstructure as compared to the $\alpha$ lamellar structure in EBM produced Ti64 samples. Vilaro et al. and Facchini et al. also reported higher yield and ultimate strengths for as-fabricated SLM Ti64 specimens $[16,17]$. Though the yield strength and tensile strength of EBM produced Ti64 samples are low when compared to the SLM produced Ti64 samples, the strain at break is higher indicating good ductility. The tensile strength values reported by Facchini et al [13], Al-Bermani [12] and Chahine et al [18] for EBM produced Ti64 samples are similar to the results obtained in the current study. However the tensile strength values reported by Murr et al. are slightly higher than the values reported in this study [6]. The ultimate tensile strengths for both SLM and EBM produced Ti64 samples are only marginally higher than their yield strengths indicating the work hardening rate beyond the yield point is low.

Table C.1: Tensile Results for SLM Produced and EBM Produced Ti64 Alloy Samples

\begin{tabular}{|c|c|c|c|}
\hline & $\begin{array}{c}\text { Stress at Yield } \\
\text { [Offset } 0.2 \% \text { ] }(\mathrm{MPa})\end{array}$ & $\begin{array}{l}\text { Ultimate tensile stress } \\
(\mathrm{MPa})\end{array}$ & Strain at break $(\%)$ \\
\hline $\begin{array}{l}\text { EBM (Vertically built } \\
\text { and Machined) }\end{array}$ & $\begin{array}{c}869 \\
(\mathrm{SD}: 7.2)\end{array}$ & $\begin{array}{c}928 \\
(\mathrm{SD}: 9.8)\end{array}$ & $\begin{array}{c}9.9 \\
(\mathrm{SD}: 1.7)\end{array}$ \\
\hline $\begin{array}{l}\text { SLM (Vertically built } \\
\text { and Machined) }\end{array}$ & $\begin{array}{c}1143 \\
(\mathrm{SD}: 30)\end{array}$ & $\begin{array}{c}1219 \\
(\mathrm{SD}: 20)\end{array}$ & $\begin{array}{c}4.89 \\
(\mathrm{SD}: 0.6)\end{array}$ \\
\hline$\%$ increase & 31 & 31 & -50 \\
\hline $\begin{array}{l}\text { EBM (Horizontally } \\
\text { built and Machined) }\end{array}$ & $\begin{array}{c}899 \\
\text { (SD: } 4.7)\end{array}$ & $\begin{array}{c}978 \\
(\mathrm{SD}: 3.2)\end{array}$ & $\begin{array}{c}9.5 \\
\text { (SD: } 1.2)\end{array}$ \\
\hline $\begin{array}{l}\text { SLM (Horizontally } \\
\text { built and Machined) }\end{array}$ & $\begin{array}{c}1195 \\
(\mathrm{SD}: 19)\end{array}$ & $\begin{array}{c}1269 \\
(\mathrm{SD}: 9)\end{array}$ & $\begin{array}{c}5 \\
(\mathrm{SD}: 0.5)\end{array}$ \\
\hline$\%$ increase & 33 & 30 & -47 \\
\hline $\begin{array}{l}\text { ASM } \\
\text { Handbook(1993) } \\
\text { (Cast and annealed) }\end{array}$ & 885 & 930 & \\
\hline
\end{tabular}




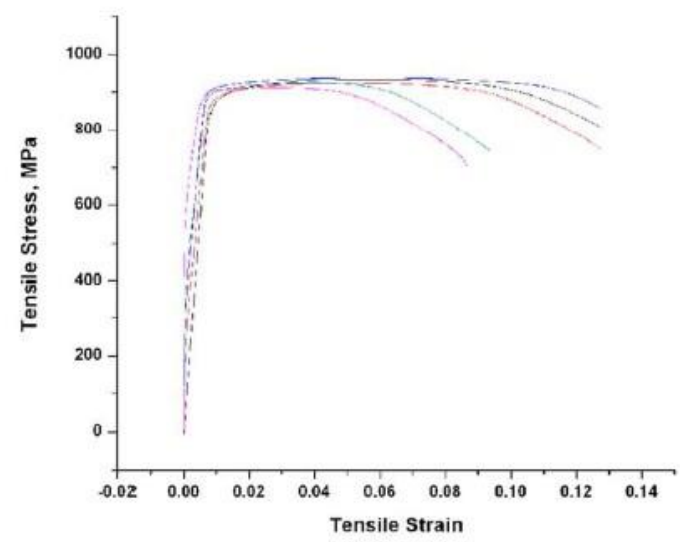

(a)

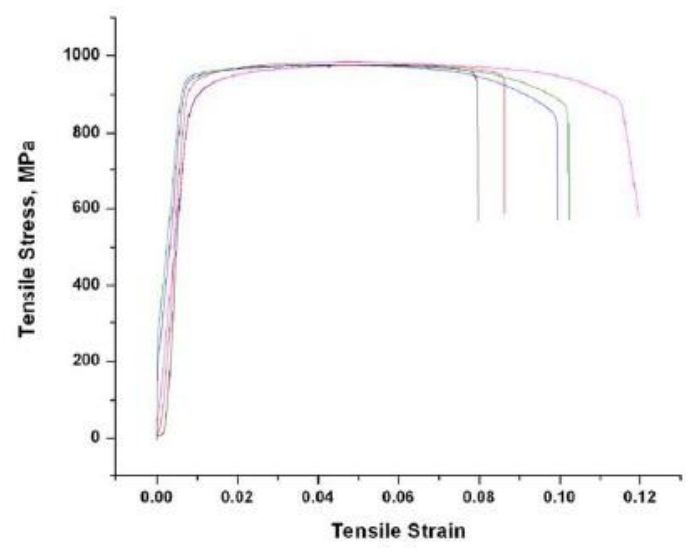

(c)

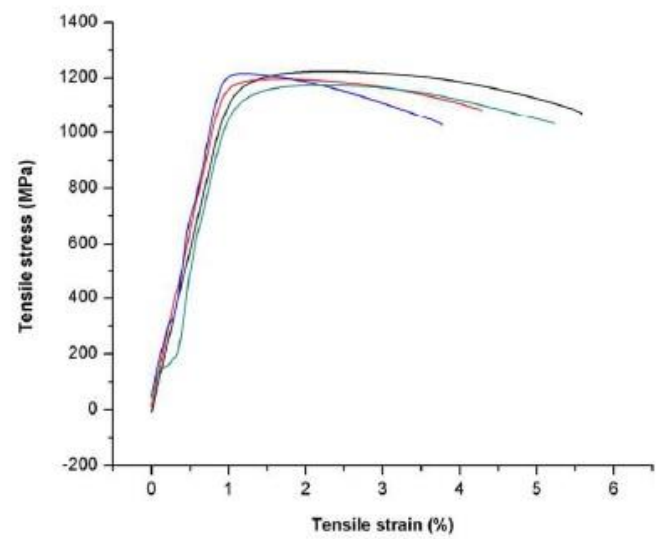

(b)

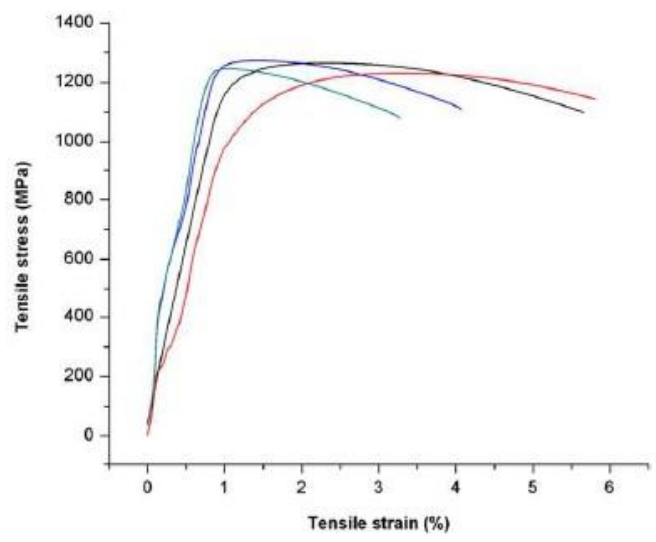

(d)

Figure C.11. Stress-Strain plots of Ti64 samples. a) EBM specimen built in vertical orientation; b) SLM specimen built in vertical orientation; c) EBM specimen built in horizontal orientation; d) SLM specimen built in horizontal orientation.

The tensile test results are in conformation with the bulk hardness tests. The Rockwell hardness test resulted in higher hardness for SLM processed Ti64 (HRC: 41) compared to the hardness of EBM processed Ti64 (HRC: 33).

Martensitic microstructure in Ti64 results in high strength and low ductility. Because of the low ductility, the SLM Ti64 samples failed at lower strain values. Ductility of SLM produced Ti64 samples can be improved by proper post-heat treatment which decomposes the harder martensitic phase to softer $\alpha$ phase [14]. The tensile properties of EBM produced Ti64 sample are greatly influenced by the alpha lath width and alpha colony 
size in the microstructure. Coarsened $\alpha$ phase and larger $\alpha$ colony size results in the reduction of tensile strength [12]. In EBM processes the morphology of the $\alpha$ phase is primarily determined by the temperature maintained in the build chamber. A slight increase in build chamber temperature can cause coarsening of the $\alpha$ phase and thereby causes a reduction in tensile strength. Another factor which affects the tensile properties is a chemical difference. This becomes a major concern when the Ti64 powder is recycled many times. Though there would not be any change in the composition of major alloying elements like $\mathrm{V}$ and $\mathrm{Al}$, over time the Ti64 powder picks up oxygen due to the high affinity of Ti for oxygen [12].

\section{C.3.5 Fatigue properties}

S-N curves illustrating the fatigue behavior of SLM produced Ti64 and EBM produced Ti64 are shown in Figure C.12. For SLM produced Ti64 samples a fatigue limit of $550 \mathrm{MPa}$ was observed (Figure C.12a). A comparison with MMPDS (Metallic Materials Properties Development and Standardization Handbook, Battelle Memorial Institute, Columbus, Ohio, April 2010) fatigue data shows better fatigue performance for Ti64 specimens built using SLM compared to Ti64 that is cast and annealed (430 MPa). However, the fatigue performance of EBM produced Ti64 samples was inferior (Figure C.12b, fatigue limit: $340 \mathrm{MPa}$ ) as compared to the SLM produced Ti64 and the MMPDS data. The better fatigue strength properties of SLM produced Ti64 can be attributed to the martensitic phase. Martensite impedes dislocation motion and thus leads to a strengthening effect. Because of this strengthening effect, the total strain amplitude introduced during fatigue testing causes smaller plastic strain. 
(a)

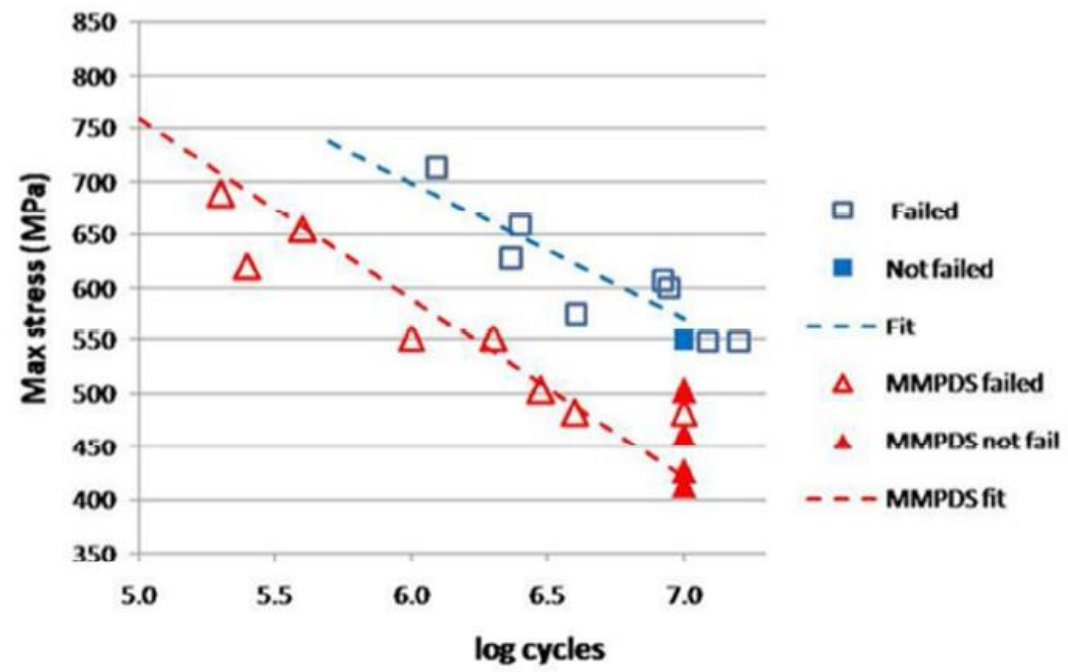

(b)

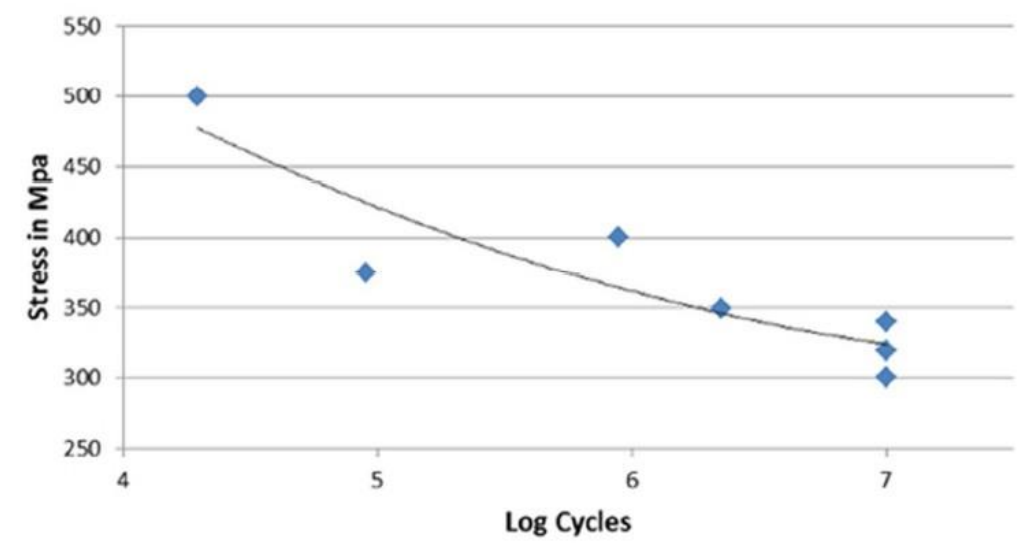

Figure C.12. $S-N$ curve showing fatigue behavior of Ti64 samples. a) SLM; b) EBM.

\section{C.3.6 Fracture behavior}

\section{Tensile Fracture}

Representative fractographs of the tensile fracture surface of SLM and EBM produced Ti64 samples are shown in Figure C.13. SLM produced Ti64 sample fracture surfaces exhibited a mixed mode of brittle and ductile fracture (Fig C.13a and C.13b) showing predominantly cleavage facets. Cleavage fracture is a low energy brittle fracture which propagates along low index crystallographic planes [19]. This fracture is characterized by flat, cleavage facets. River markings separating the facets result from the 
propagation of the crack on a number of planes of different levels [20]. The fracture surface of EBM produced Ti64 sample is characterized by transgranular ductile dimple tearing resulting from the coalescence of microvoids (Fig C.13c and C.13d). A healthy population of fine dimples at the tensile fracture surface indicates the extent of plastic deformation.

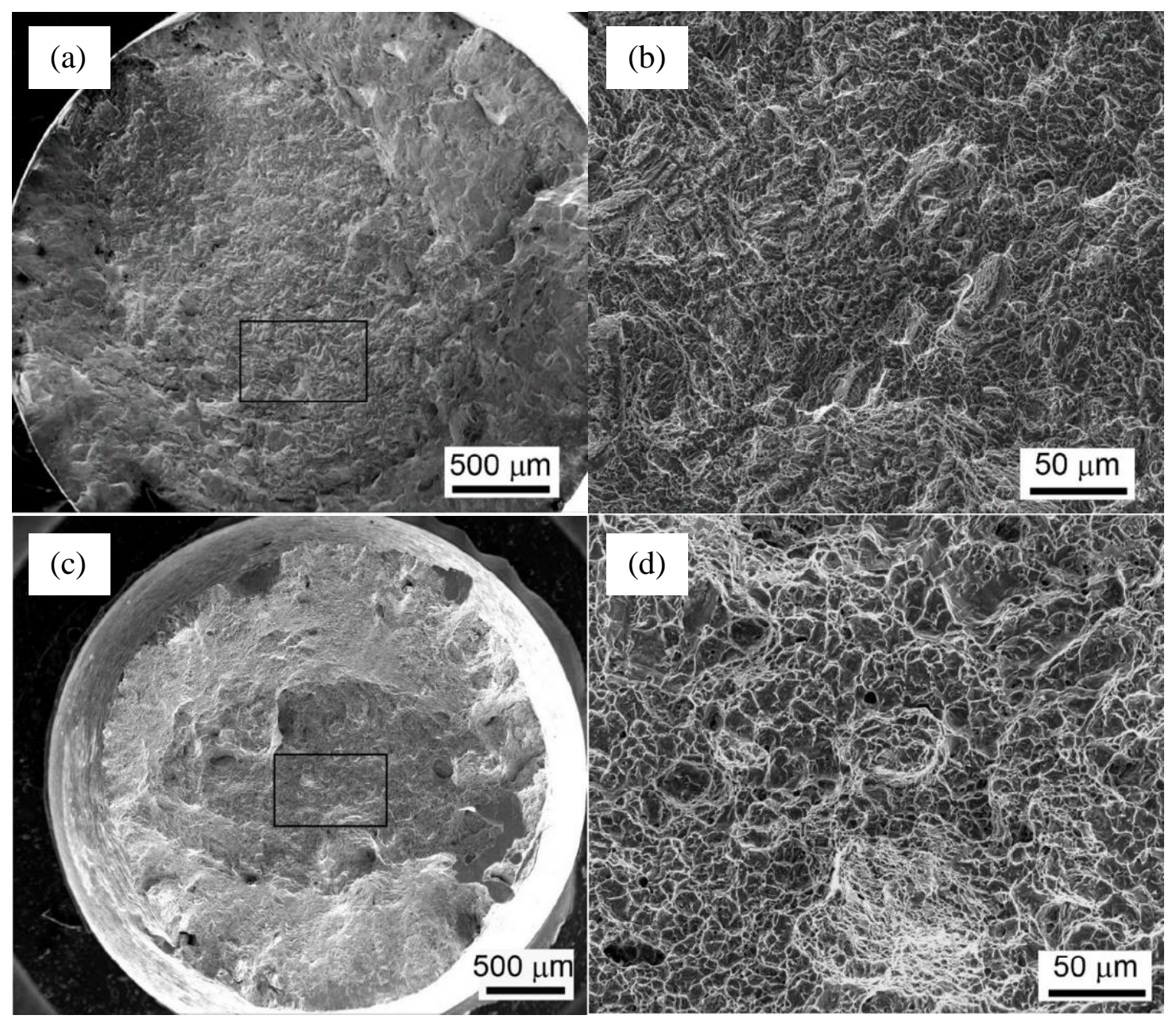

Figure C.13. SEM- SE images of tensile fracture surfaces. a) Overall view of SLM produced Ti64 tensile fracture surface; b) enlarged view from the boxed region in 'a'; c) overall view of EBM produced Ti64 tensile fracture surface; d) enlarged view from the boxed region in 'c'.

\section{Fatigue fracture}

Figure C.14 shows the fatigue fracture surfaces of SLM produced Ti64 and EBM produced Ti64. In SLM produced Ti64 samples (Figure C.14a, C.14b) the crack initiated 
from an internal defect and propagated radially outwards. Three distinct regions show crack initiation, steady crack growth and overload regions typical for fatigue fracture. The crack propagation looks more tortuous as it propagated through multiple crystallographic planes. The fracture surface of EBM produced Ti64 samples appear normal, with a characteristic fatigue fracture surface (Fig C.14c). The crack initiation site is characterized by microscopically smooth facets away from the surface as evident from Figure C.14d.

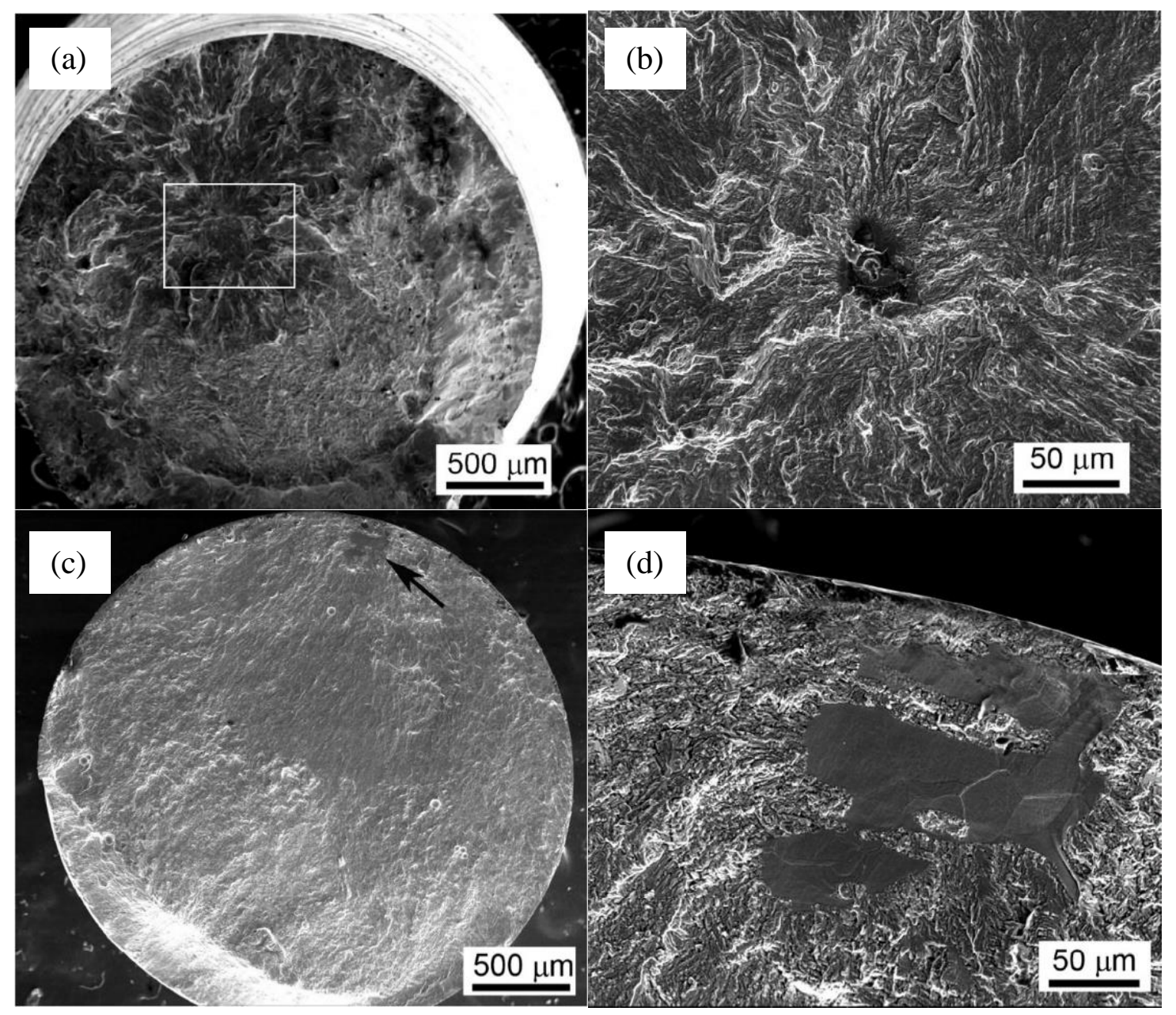

Figure C.14. SEM- SE images of fatigue fracture surfaces. a) Overall view of SLM produced Ti64 fracture surface; b) enlarged view from the boxed region in ' $a$ '; c) overall view of EBM produced Ti64 fracture surface; d) enlarged view from the arrow pointed region in 'c'. 
It can also be noted that the crack initiation site has multiple facets which could possibly be due to crack initiation occurring at the $\alpha$ colony boundaries and shear across neighboring $\alpha$ colonies. This indicates crack initiation is not caused by the classical slip band intrusion/extrusion mechanism. Therefore, one common feature that can be seen between the fatigue fracture surfaces of SLM and EBM produced Ti64 samples is that the crack initiation occurred from the interior or the sub surface and not from the external surface. In general, for metallic materials fatigue crack initiation occurs at the surface if the sample is free from large internal defects. Previous studies show that this may not be true in the case of Ti64 with dual phase microstructures. In $\alpha / \beta$ titanium alloys, cracks tend to initiate either at the surface or at the subsurface or at the impingement of slip bands within the alpha lamellae of a fully lamellar microstructure. They can also initiate at the boundaries separating the $\alpha$ and $\beta$ phase [21].

Fatigue behavior of Ti64 is greatly influenced by its microstructure. Depending on the process conditions the morphology and volume fraction of $\alpha$ and $\beta$ constituents in Ti64 changes. The microstructure of Ti64 can exist in forms such as bi-modal, equiaxed, lamellar $\alpha$ and $\beta$, and martensitic $\alpha$ structures. All these microstructures can result in different fatigue properties. As observed earlier, Ti64 processed by SLM resulted in martensitic $\alpha$ and EBM resulted in lamellar $\alpha$ with smaller amount of $\beta$. Ivanova et al. observed that in the high cycle fatigue regime the preferred crack initiation sites are within the alpha grains in an equiaxed grain structure. The crack nucleates along the slip bands within the alpha grain and results in cleavage fracture [22]. For a bimodal microstructure the crack initiates by the cracking of suitably oriented $\alpha$ grains and subsequent growth along the planar slip bands within interconnected $\alpha$ grains. Crack initiation occurs by cross- 
colony slip-band fracture for the lamellar microstructure [23]. Oh et al. showed that fatigue crack initiation occurs mainly at the prior $\beta$ grain boundary for an electron beam welded and then annealed Ti64 sample. They also observed that the large micropores present in the weld act as fatigue crack initiation sites. However, the micropores did not have any effect on crack propagation [24]. In titanium alloys, an increase in oxygen content can also lead to the embrittlement of the alpha phase. Among the different characteristic microstructures exhibited by Ti64, lamellar structures are more prone to crack initiation as compared to equiaxed or bi-modal structures. But for crack propagation, lamellar structures offer greater resistance to crack growth than equiaxed structures. In high cycle fatigue, crack initiation forms the major part of the fatigue life. To increase the resistance to fatigue crack nucleation, the maximum dislocation slip length in the microstructure should be reduced. Generally, a fatigue crack nucleates due to irreversible slip bands within the longest crystallographic planes available in the microstructure. Consequently, coarse lamellar microstructures with colonies of aligned $\alpha$ and extended planar- slips running across these colonies often have less resistance to crack nucleation when compared to finegrained equi-axed and acicular martensitic microstructures [23]. Therefore, the inferior fatigue strength of EBM samples could be attributed to its lamellar microstructure rather than due to the presence of micorpores or voids.

\section{C.4 Summary}

In this study an attempt was made to highlight the differences between SLM and EBM processes concerning Ti64. A clear difference in surface conditions, microstructure, tensile properties and fatigue properties are evident. The primary difference between the 
processes boils down to the cooling rate. The same material processed using SLM and EBM can have different properties. Irrespective of the processes, what matters is the suitability of a final part for a specific application. Ti64 is considered for very wide range of applications from biomedical to aerospace. The property requirements for a part produced for biomedical applications may differ from a part produced for aerospace applications. As discussed earlier, processing of Ti64 with SLM and EBM results in microstructures containing $\alpha, \alpha$ and $\beta$ phases. With respect to the volume fraction of these phases and their morphology the resulting properties will vary to a certain extent. For instance, the higher hardness possessed by $\alpha$ phases may be beneficial for certain applications and on the other hand it may be detrimental for some other applications. Therefore, selection of a particular process, whether SLM or EBM, primarily depends on the requirements for end-use. For that, one should have a proper understanding of the structure-property relationship of the material chosen.

\section{C.5 Conclusions}

This comparative study on SLM and EBM processes when using Ti64 as the build material has resulted in the following conclusions:

1. The surface finish of SLM produced samples is better than EBM processed samples. Relatively thick layers in EBM cause more pronounced 'stairstep effects' and larger particle size powders adhering to the surface result in rougher surfaces.

2. The SLM produced and EBM produced samples resulted in two different microstructures for Ti64. SLM produced Ti64 resulted in martensitic á microstructures and EBM produced Ti64 resulted in an $\alpha$ phase with $\beta$ separating the $\alpha$ lamellae. 
3. SLM produced Ti64 samples have higher tensile strength than EBM produced samples. But EBM produced samples have higher ductility. Higher tensile strength of SLM samples is attributed to the martensitic $\alpha$ microstructure and the higher ductility in EBM produced samples is attributed to the lamellar $\alpha$ phase.

4. The SLM produced samples showed a fatigue limit of $550 \mathrm{MPa}$ whereas EBM produced samples showed a fatigue limit of $340 \mathrm{MPa}$. The inferior fatigue limit observed for EBM is because of the lamellar phase microstructure.

5. Selecting between SLM and EBM for fabrication of a specific Ti64 part depends on the application requirements as both result in mechanical properties suitable for many applications.

Acknowledgements

The authors acknowledge the Office of Naval Research (ONR), USA for support through grant \#'s N00014-09-1-0147, N00014-10-1-0800, and N00014-11-1-0689.

\section{References}

1. F. Abe, K. Osakada, M. Shiomi, K. Uematsu, and M. Matsumoto. The manufacturing of hard tools from metallic powders by selective laser melting. J. Mater. Process. Tech., 111,2001, p. 210-213

2. S.M. Gaytan, L.E. Murr, E. Martinez, J.L. Martinez, B.I. Machado, D.A. Ramirez, F. Medina, S. Collins, and R.B. Wicker. Comparison of Microstructures and Mechanical Properties for Solid and Mesh Cobalt-Base Alloy Prototypes Fabricated by Electron Beam Melting. Metall. Mater. Trans. A, 41 A, 2010, p. 3216-3227

3. E. Brinksmeier, G. Levy, D. Meyer, A.B. Spierings. Surface integrity of selective-lasermelted components. CIRP Annals - Manufacturing Technology, 59(1), 2010, p. 601-606

4. J.P. Kruth, G. Levy, F. Klocke, T.H.C. Childs, Consolidation phenomena in laser and powder-bed based layered manufacturing. CIRP Annals - Manufacturing Technology, 56, 2007, p. 730-759 
5. M.F Zah, and S. Lutzmann. Modelling and simulation of electron beam melting. Prod. Eng. Res. Devel., 4, 2010, p.15-23

6. L.E. Murr, E.V Esquivel, S.A. Quinones, S.M. Gaytan, M.I. Lopez, E.Y. Martinez, F. Medina, D.H. Hernandez, E. Martinez, J.L. Martinez, S.W. Stafford, D.K. Brown, T. Hoppe, W. Meyers, U. Lindhe, and R.B. Wicker. Microstructures and mechanical properties of electron beam-rapid manufactured $\mathrm{Ti}-6 \mathrm{Al}-4 \mathrm{~V}$ biomedical prototypes compared to wrought Ti-6Al-4V. Mater. Charact., 60, 2009, p. 96-109

7. L. Thijs, F. Verhaeghe, T. Craeghs, J.V. Humbeeck, and J.P Kruth. A study of the microstructural evolution during selective laser melting of Ti-6Al-4V. Acta Mater., 58,2010, p. 3303-12

8. B. Song, S. Dong, B. Zhang, H. Liao, and C. Coddet. Effects of processing parameters on microstructure and mechanical property of selective laser melted Ti6Al4V. Mater. Des., 35, 2012, p.120-125

9. I. Yadroitsev, P. Bertrand, and I. Smurov. Parametric analysis of the laser melting process. Appl. Surf. Sci., 253(19), 2007, p.8064-8069.

10. R. Morgan, C.J. Sutcliffe, and W. O'Neill. Density analysis of direct metal laser remelted 316L stainless steel cubic primitives. J. Mater. Sci., 39(4), 2004, p.1195-205

11. E. Yasa, J. Deckers, and J.P. Kruth. The investigation of the influence of laser remelting on density, surface quality and microstructure of selective laser melting parts. Rapid prototyping J., 17(5), 2011, p. 312-327

12. S. S. Al-Bermani, M. L. Blackmore, W. Zhang, and I. Todd. The Origin of Microstructural Diversity, Texture, and Mechanical Properties in Electron Beam Melted Ti-6Al-4V. Metall. Mater. Trans. A., 41A, 2010, p. 3422-3432

13. L. Facchini, E. Magalini, P. Robotti, and Molinari A. Microstructure and mechanical properties of Ti-6Al-4V produced by electron beam melting of pre-alloyed powders. Rapid Prototyping J., 15(3), 2009, p. 171-178.

14. B. Vrancken., L. Thijis, J. P. Kruth, and J. V. Humbeeck. Heat treatment of Ti6Al4V produced by selective laser melting- Microstructure and mechanical properties. J. Alloy. Compd., 541, 2012, p. $177-185$

15. ASM Handbook Vol.1 (page no. 2071), ASM International, Materials Park, Ohio

16. T. Vilaro, C. Colin, and J.D Bartout. As-fabricated and heat treated microstructures of the Ti-6Al-4V alloy processed by selective laser melting. Metall. Mater. Trans. A., 42, 2011, p. 3190 
17. L. Facchini, E. Magalini, P. Robotti, A. Molinari, S. Hogess, and K. Wissenbach. Ductility of Ti-6Al-4V alloy produced by selective laser melting of pre-alloyed powders. Rapid Prototyping J. 16(6), 2010, p. 450-459.

18. G. Chahine, M. Koike, T. Okabe, P. Smith, and R. Kovacevic, The Design and Production of Ti-6Al-4V ELI Customized Dental Implants. JOM. 60 (11), 2008, p. 50-55

19. G.E Dieter. Mechanical Metallurgy: McGraw Hill Co. New York, 1986.

20. M. Erdogan and S. Tekeli. The effect of martensitic particle size on tensile fracture of surface-carburized AISI 8620 steel with dual phase core microstructure. Mater. Des., 23, 2002, p. 597-604

21. T. S. Srivatsana, M. Kuruvilla, and L. Park. A study at understanding the mechanisms governing the high cycle fatigue and final fracture behavior of the titanium alloy: Ti-4Al2.5V. Mater. Sci. Eng. A., 527, 2010, 435-448

22. S. G. Ivanova, R.R. Biederman, and R.D. Sisson Jr. Investigation of fatigue crack initiation in Ti-6Al-4V during tensile-tensile fatigue. J. Mater. Eng. Perform, 11(2), 2002, p. $226-31$

23. R.K. Nalla, B.L. Boyce, J.P Campbell, J.O Peters, and R.O Ritchie. Influence of microstructure on high-cycle fatigue of Ti-6Al-4V: Bimodal vs. lamellar structures. Metall. Mater. Trans. A., 33A, 2002, p. 899-918

24. J. Oh, N.J Kim, S. Lee, W. Lee. Correlation of fatigue properties and microstructure in investment cast Ti-6Al-4V. Mater. Sci. Eng. A., 340, 2003, p.232-242 


\section{APPENDIX D \\ PERMISSIONS}

This appendix includes all required permissions for publication of the papers presented as chapters and appendices of this dissertation. 


\title{
Gong,Haijun
}

\author{
From: \\ Sent: \\ To: \\ Khalidrafi < khalidrafi@gmail.com> \\ Sunday, November 24, 2013 4:01 AM \\ Subject: \\ Gong, Haijun \\ Re: Permission for Paper Inclusion in Dissertation \\ Haijun, \\ I am fully agreed to use the manuscripts mentioned in the email for your thesis. \\ All the very best for your defence. \\ Regards \\ Rafi \\ Sent from my iPhone \\ On 24-Nov-2013, at 3:21 am, "Gong,Haijun" <haijun.gong@louisville.edu> wrote:
}

\section{Dear Dr. H. Khalid Rafi,}

I am preparing my dissertation in the Department of Industrial Engineering at University of Louisville. I hope to defense and complete my degree in the fall of 2013.

This is a multiple paper-based dissertation, which will include the paper listed below. I am requesting your permission to include the material just as it appeared in the paper.

1. Haijun Gong, Khalid Rafi, Thomas Starr, Brent Stucker. "The Effects of Processing Parameters on Defect Regularity in Ti-6Al-4V Parts Fabricated by Selective Laser Melting and Electron Beam Melting", Solid Freeform Fabrication Symposium 2013, 424439.

2. Haijun Gong, Khalid Rafi, N.V. Karthik, Thomas Starr, Brent Stucker. "Defect Morphology of Ti-6Al-4V Parts Fabricated by Selective Laser Melting and Electron Beam Melting", Solid Freeform Fabrication Symposium 2013, 440-453.

3. Haijun Gong, N.V. Karthik, Khalid Rafi, Thomas Starr, Brent Stucker. "Micro-CT Evaluation of Stochastic Defects Fabricated by SLM and EBM", not yet submitted.

4. Haijun Gong, Khalid Rafi, Hengfeng Gu, Thomas Starr, Brent Stucker. "Mechanical Properties of SLM- and EBM-Produced Ti-6Al-4V with Defects", not yet submitted.

5. Haijun Gong, Khalid Rafi, Thomas Starr, Brent Stucker. "Effect of Defects on Fatigue Tests of As-Built Ti-6Al-4V Parts Fabricated by Selective Laser Melting", Solid Freeform Fabrication Symposium 2012, 499-506.

6. H. Khalid Rafi, N.V. Karthik, Haijun Gong, Thomas L. Starr, Brent E. Stucker. "Microstructures and Mechanical Properties of Ti6Al4V Parts Fabricated by Selective Laser Melting and Electron Beam Melting", Journal of Materials Engineering and Performance, Article in press.

Please indicate your approval by replying this email, and attach any other form or instruction necessary to confirm permission. If you have any questions, please feel free to contact me. Thank you!

Best regards,

Haijun Gong

Dept. of Industrial Engineering

University of Louisville

Louisville, KY 40292 
Date $11 / 17 / 2013$

$\begin{array}{ll}\begin{array}{l}\text { Name } \\ \text { Address }\end{array} & \begin{array}{l}\text { Haijun Gong } \\ \text { Department of Industrial Engineering } \\ \text { J.B. Speed School of Engineering }\end{array} \\ & \begin{array}{l}\text { University of Louisville } \\ \text { Louisville, KY } 40292\end{array} \\ & \text { (502)852-2542 } \\ \text { Phone } & \text { haijun.gong@louisville.edu }\end{array}$

Dear N.V. Karthik,

I am preparing my dissertation in the Department of Industrial Engineering at University of Louisville. I hope to defense and complete my degree in the fall of 2013.

This is a multiple paper-based dissertation, which will include the paper listed below. I am requesting your permission to include the material just as it appeared in the paper.

Please indicate your approval by signing in the space below, and attach any other form or instruction necessary to confirm permission. If you have any questions, please feel free to contact me. Thank you!

Best regards,

Haijun Gong

I hereby give permission to HAIJUN GONG to reprint the requested articles in his dissertation.

Haijun Gong, Khalid Rafi, N.V. Karthik, Thomas Starr, Brent Stucker. "Defect Morphology of Ti-6Al-4V Parts Fabricated by Selective Laser Melting and Electron Beam Melting", Solid Freeform Fabrication Symposium 2013, 440-453.

Haijun Gong, N.V. Karthik, Khalid Rafi, Thomas Starr, Brent Stucker. "Micro-CT Evaluation of Stochastic Defects Fabricated by SLM and EBM", not yet submitted.

H. Khalid Rafi, N.V. Karthik, Haijun Gong, Thomas L. Starr, Brent E. Stucker. "Microstructures and Mechanical Properties of Ti6Al4V Parts Fabricated by Selective Laser Melting and Electron Beam Melting", Journal of Materials Engineering and Performance, Article in press.

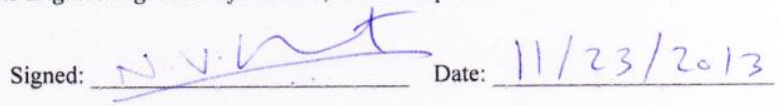


Date $11 / 17 / 2013$

$\begin{array}{ll}\text { Name } & \begin{array}{l}\text { Haijun Gong } \\ \text { Department of Industrial Engineering } \\ \text { J.B. Speed School of Engineering }\end{array} \\ & \begin{array}{l}\text { University of Louisville } \\ \text { Louisville, KY } 40292\end{array} \\ & \text { (502)852-2542 } \\ \text { Phone } & \text { haijun.gong@louisville.edu }\end{array}$

Dear Hengfeng Gu,

I am preparing my dissertation in the Department of Industrial Engineering at University of Louisville. I hope to defense and complete my degree in the fall of 2013.

This is a multiple paper-based dissertation, which will include the paper listed below. I am requesting your permission to include the material just as it appeared in the paper.

Please indicate your approval by signing in the space below, and attach any other form or instruction necessary to confirm permission. If you have any questions, please feel free to contact me. Thank you!

Best regards,

Haijun Gong

I hereby give permission to HAIJUN GONG to reprint the requested articles in his dissertation.

Haijun Gong, Khalid Rafi, Hengfeng Gu, Thomas Starr, Brent Stucker. "Mechanical Properties of SLMand EBM-Produced Ti-6Al-4V with Defects", not yet submitted.

Signed: Hevfyy Cu Date: $11 / 22 / 2013$. 
Date $11 / 17 / 2013$

$\begin{array}{ll}\text { Name } & \text { Haijun Gong } \\ \text { Address } & \begin{array}{l}\text { Department of Industrial Engineering } \\ \text { J.B. Speed School of Engineering } \\ \text { University of Louisville }\end{array} \\ & \begin{array}{l}\text { Louisville, KY } 40292 \\ (502) 852-2542\end{array} \\ \text { Phone } & \text { haijun.gong@louisville.edu }\end{array}$

Dear Dr. Thomas Starr,

I am preparing my dissertation in the Department of Industrial Engineering at University of Louisville. I hope to defense and complete my degree in the fall of 2013.

This is a multiple paper-based dissertation, which will include the paper listed below. I am requesting your permission to include the material just as it appeared in the paper.

Please indicate your approval by signing in the space below, and attach any other form or instruction necessary to confirm permission. If you have any questions, please feel free to contact me. Thank you!

Best regards,

Haijun Gong

I hereby give permission to HAIJUN GONG to reprint the requested articles in his dissertation.

Haijun Gong, Khalid Rafi, Thomas Starr, Brent Stucker. "The Effects of Processing Parameters on Defect Regularity in Ti-6Al-4V Parts Fabricated by Selective Laser Melting and Electron Beam Melting", Solid Freeform Fabrication Symposium 2013, 424-439.

Haijun Gong, Khalid Rafi, N.V. Karthik, Thomas Starr, Brent Stucker. "Defect Morphology of Ti-6Al-4V Parts Fabricated by Selective Laser Melting and Electron Beam Melting", Solid Freeform Fabrication Symposium 2013, 440-453.

Haijun Gong, N.V. Karthik, Khalid Rafi, Thomas Starr, Brent Stucker. "Micro-CT Evaluation of Stochastic Defects Fabricated by SLM and EBM", not yet submitted.

Haijun Gong, Khalid Rafi, Hengfeng Gu, Thomas Starr, Brent Stucker. "Mechanical Properties of SLMand EBM-Produced Ti-6Al-4V with Defects", not yet submitted.

Haijun Gong, Khalid Rafi, Thomas Starr, Brent Stucker. "Effect of Defects on Fatigue Tests of As-Built Ti-6Al-4V Parts Fabricated by Selective Laser Melting", Solid Freeform Fabrication Symposium 2012, 499-506.

H. Khalid Rafi, N.V. Karthik, Haijun Gong, Thomas L. Starr, Brent E. Stucker. "Microstructures and Mechanical Properties of Ti6Al4V Parts Fabricated by Selective Laser Melting and Electron Beam Melting", Journal of Materials Engineering and Performance, Article in press.

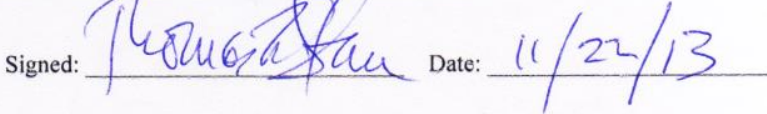




\section{SPRINGER LICENSE TERMS AND CONDITIONS}

Nov 18,2013

This is a License Agreement between Haijun Gong ("You") and Springer ("Springer") provided by Copyright Clearance Center ("CCC"). The license consists of your order details, the terms and conditions provided by Springer, and the payment terms and conditions

All payments must be made in full to CCC. For payment instructions, please see information listed at the bottom of this form.

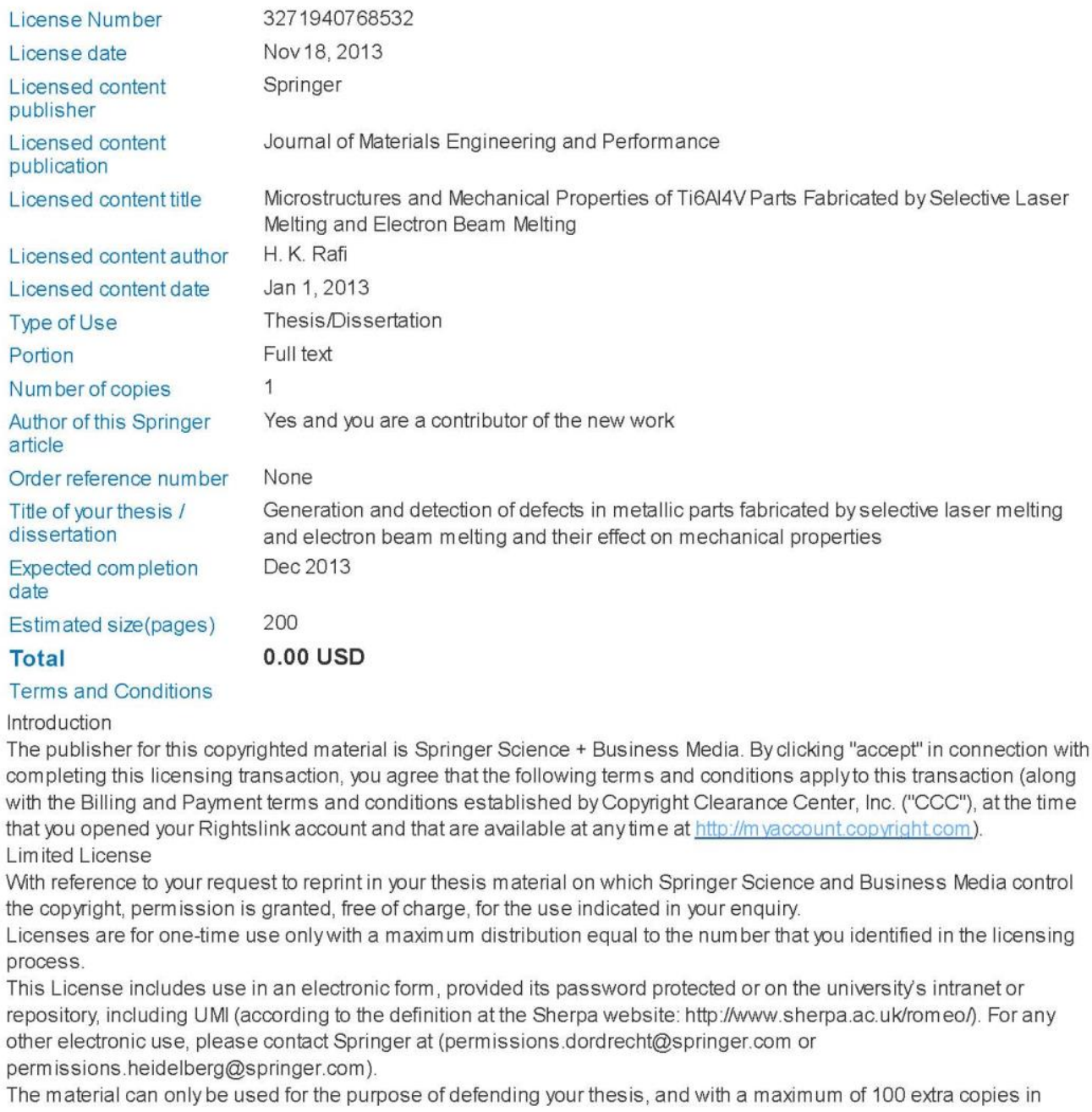

This License includes use in an electronic form, provided its password protected or on the university's intranet or repository, including UMl (according to the definition at the Sherpa website: http://WwW.sherpa.ac.uk/romeo/). For any other electronic use, please contact Springer at (permissions.dordrecht@springer.com or permissions.heidelberg@springer.com).

The material can only be used for the purpose of defending your thesis, and with a maximum of 100 extra copies in 
paper.

Although Springer holds copyright to the material and is entitled to negotiate on rights, this license is only valid, subject to a courtesy information to the author (address is given with the article/chapter) and provided it concerns original material which does not carry references to other sources (if material in question appears with credit to another source, authorization from that source is required as well).

Perm ission free of charge on this occasion does not prejudice any rights we might have to charge for reproduction of our copyrighted material in the future. Altering/Modifying Material: Not Permitted

You may not alter or modify the material in any manner. Abbreviations, additions, deletions and/or any other alterations shall be made only with prior written authorization of the author(s) and/or Springer Science + Business Media. (Please contact Springer at (permissions.dordrecht@springer.com or permissions.heidelberg@springer.com) Reservation of Rights

Springer Science + Business Media reserves all rights not specifically granted in the com bination of (i) the license details provided by you and accepted in the course of this licensing transaction, (ii) these terms and conditions and (iii) CCC's Billing and Payment terms and conditions.

Copyright Notice:Disclaimer

You must include the following copyright and permission notice in connection with any reproduction of the licensed material: "Springer and the original publisher/journal title, volume, year of publication, page, chapter/article title, name(s) of author(s), figure number(s), original copyright notice) is given to the publication in which the material was originally published, byadding; with kind permission from Springer Science and Business Media" Warranties: None

Example 1: Springer Science + Business Media makes no representations or warranties with respect to the licensed material.

Example 2: Springer Science + Business Media makes no representations or warranties with respect to the licensed material and adopts on its own behalf the limitations and disclaimers established by CCC on its behalf in its Billing and Payment terms and conditions for this licensing transaction. Indemnity

You hereby indemnify and agree to hold harmless Springer Science + Business Media and CCC, and their respective officers, directors, em ployees and agents, from and against any and all claims arising out of your use of the licensed material other than as specifically authorized pursuant to this license.

No Transfer of License

This license is personal to you and may not be sublicensed, assigned, or transferred by you to any other person without Springer Science + Business Media's written permission.

No Amendment Except in Writing

This license may not be amended except in a writing signed by both parties (or, in the case of Springer Science +

Business Media, by CCC on Springer Science + Business Media's behalf)

Objection to Contrary Terms

Springer Science + Business Media hereby objects to any terms contained in any purchase order, acknowledgment, check endorsement or other writing prepared by you, which terms are inconsistent with these terms and conditions or CCC's Billing and Payment terms and conditions. These terms and conditions, together with CCC's Billing and Payment terms and conditions (which are incorporated herein), comprise the entire agreement between you and Springer Science + Business Media (and CCC) concerning this licensing transaction. In the event of any conflict between your obligations established by these terms and conditions and those established by CCC's Billing and Payment terms and conditions, these terms and conditions shall control.

Jurisdiction

All disputes that may arise in connection with this present License, or the breach thereof, shall be settled exclusively by arbitration, to be held in The Netherlands, in accordance with Dutch law, and to be conducted under the Rules of the 'Netherlands Arbitrage Instituut' (Netherlands Institute of Arbitration).OR

All disputes that may arise in connection with this present License, or the breach thereof, shall be settled exclusively by arbitration, to be held in the Federal Republic of Germany, in accordance with German law.

Other terms and conditions:

v1.3

If you would like to pay for this license now, please remit this license along with your payment made payable to "COPYRIGHT CLEARANCE CENTER" otherwise you will be invoiced within $\mathbf{4 8}$ hours of the license date. Payment should be in the form of a check or money order referencing your account number and this invoice number RLNK501161614. Once you receive your invoice for this order, you may pay your invoice by credit card. Please follow instructions 
provided at that time.

Make Payment To:

Copyright Clearance Center

Dept 001

P.O. Box 843006

Boston, MA 02284-3006

For suggestions or comments regarding this order, contact RightsLink Customer Support: customercare@copyright.com or +1-877-622-5543 (toll free in the US) or +1-978-646-2777.

Gratis licenses (referencing $\$ 0$ in the Total field) are free. Please retain this printable license for your reference. No payment is required. 


\section{CURRICULUM VITA}

Name:

Address:

Education:

Awards:

Publications:
Haijun Gong

Department of Industrial Engineering

J.B. Speed School of Engineering

University of Louisville

Louisville, KY 40292

Ph.D., Industrial Engineering

University of Louisville, Louisville, KY

2010-2013

M.S., Mechanical Engineering

Harbin Institute of Technology, Harbin, China

2004-2006

B.E., Mechanical Engineering

Harbin University of Science and Technology, Harbin, China

2000-2004

Manufacturing \& Logistics Network Academic Scholarship

Greater Louisville Inc., Louisville, 2011

Additive Manufacturing Research Scholarship

University of Louisville, 2010-2013

"Defect Morphology in Ti-6Al-4V Parts Fabricated by Selective Laser Melting and Electron Beam Melting"

H. Gong, K. Rafi, N.V. Karthik, T. Starr, B. Stucker

Solid Freeform Fabrication Symposium Proceeding 2013

Austin, TX, p.440-453, August 2013. 
"The Effects of Processing Parameters on Defect Regularity in Ti6Al-4V Parts Fabricated By Selective Laser Melting and Electron Beam Melting"

H. Gong, K. Rafi, T. Starr, B. Stucker

Solid Freeform Fabrication Symposium Proceeding 2013

Austin, TX, p.424-439, August 2013.

"Influences of Energy Density on Porosity and Microstructure of Selective Laser Melted 17-4PH Stainless Steel"

H. Gu, H. Gong, D. Pal, K. Rafi, T. Starr, B. Stucker

Solid Freeform Fabrication Symposium Proceeding 2013

Austin, TX, p.474-489, August 2013

"Microstructures and Mechanical Properties of Ti6A14V Parts Fabricated by Selective Laser Melting and Electron Beam Melting" K. Rafi, N.V. Karthik, H. Gong, T. Starr, B. Stucker

Journal of Materials Engineering and Performance. 2013

Article in Press

"Effect of Defects on Fatigue Tests of As-Built Ti-6Al-4V Parts Fabricated by Selective Laser Melting"

H. Gong, K. Rafi, T. Starr, B. Stucker

Solid Freeform Fabrication Symposium Proceeding 2012

Austin, TX, p.499-506, August 2012.

"Color Stereolithography Based on Time-pressure Dispensing Process"

H. Gong, M. Wang, Y. Wang

IEEE Int'l Conference on Mechatronics and Automation (ICMA)

Xi'an, China, p.99-103, 2010.

"Laser Cure of Photosensitive Resin Mixed with Coloring Agent"

H. Gong, Y. Wang

Journal of Huazhong Univ. of Sci. \& Tech. (Nature Science Edition), 35(Sup. I), p.121-124, 2007. 